\title{
Relocation Impacts of a Major Release from SRTC
}

by

A. Blanchard

Westinghouse Savannah River Company

Savannah River Site

Aiken, South Carolina 29808

E. A. Thompson

Westinghouse Safety Management Solutions

J. M. Thompson

Westinghouse Safety Management Solutions

This paper was prepared in connection with work done under the above contract number with the U. S.

Department of Energy. By acceptance of this paper, the publisher and/or recipient acknowledges the U.S.

Government's right to retain a nonexclusive, royalty-free license in and to any copyright covering this paper, along with the right to reproduce and to authorize others to reproduce all or part of the copyrighted paper. 
Keywords: Relocation

Emergency Planning

Retention: Lifetime

\section{Relocation Impacts of a Major Release from SRTC (U)}

Elizabeth A. Thompson

Jay M. Thompson

June 1999

Classification

\section{UNCLASSIFIED}

DOES NOT CONTAIN

UNCLASSIFIED CONTROLLED

NUCLEAR INFORMATION
ADC \&

Reviewing

Official: K.R.O'Kula

K. R. O'Kula, Managing Member Consequence Analysis Group

Date: $6 / 23 / 99$

Westinghouse Savannah River Company Technical Services Division

Aiken, SC 29808

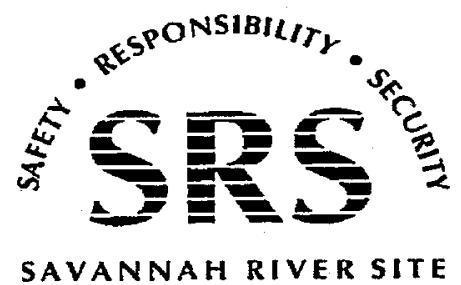

PREPARED FOR THE U.S. DEPARTMENT OF ENERGY UNDER CONTRACT NO. DE-AC09-96SR18500 
Relocation Impacts of a Major Release from SRTC (U)

\author{
Elizabeth A. Thompson
}

Jay M. Thompson

June 1999

Westinghouse Savannah River Company Technical Services Division Aiken, SC 29808

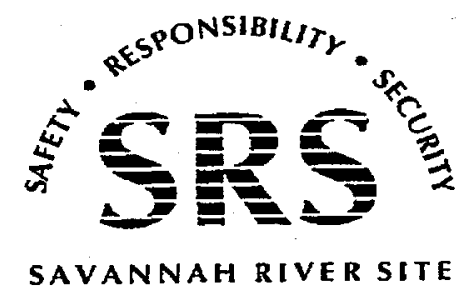

PREPARED FOR THE U.S. DEPARTMENT OF ENERGY UNDER CONTRACT NO. DE-AC09-96SR18500 


\section{DISCLAIMER}

This report was prepared as an account of work sponsored by an agency of the United States Government. Neither the United States Government nor any agency thereof, nor any of their employees, makes any warranty, express or implied, or assumes any legal liability or responsibility for the accuracy, completeness, or usefulness of any information, apparatus, product, or process disclosed, or represents that its use would not infringe privately owned rights. Reference herein to any specific commercial product, process, or service by trade name, trademark, manufacturer, or otherwise does not necessarily constitute or imply its endorsement, recommendation, or favoring by the United States Government or any agency thereof. The views and opinions of authors expressed herein do not necessarily state or reflect those of the United States Government or any agency thereof.

This report has been reproduced directly from the best available copy.

Available to DOE and DOE contractors from the Office of Scientific and Technical Information, P.O. Box 62, Oak Ridge, TN 37831; prices available from (615) 576-8401.

Available to the public from the National Technical Information Service, U.S. Department of Commerce, 5285 Port Royal Road, Springfield, VA 22161. 


\section{DISCLAIMER}

Portions of this document may be illegible in electronic image products. Images are produced from the best available original document. 
PROJECT:

DOCUMENT:

TITLE:
N/A

WSRC-TR-99-00105

Relocation Impacts of a Major Release from SRTC (U)

REVISION NUMBER:

1

\section{AUTHOR/TECHNICAL REVIEWS:}

E. A. Thompoon

E. A. Thompson, Author

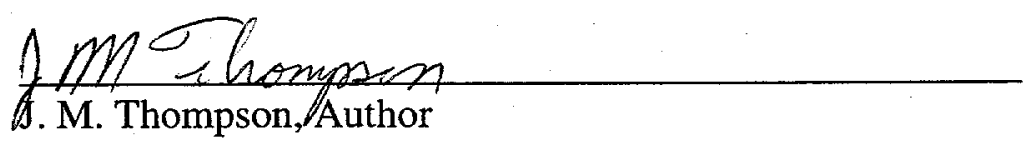

Temi Oxhla

K. R. O'Kula, Technical Reviewer

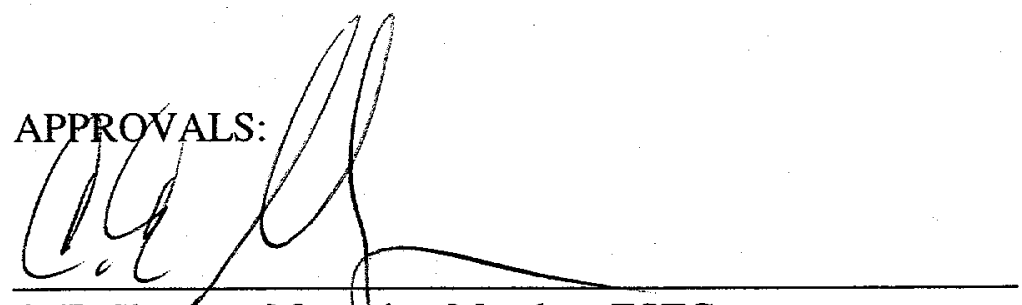

C. E. Shogren, Mana qing Member, ESTG
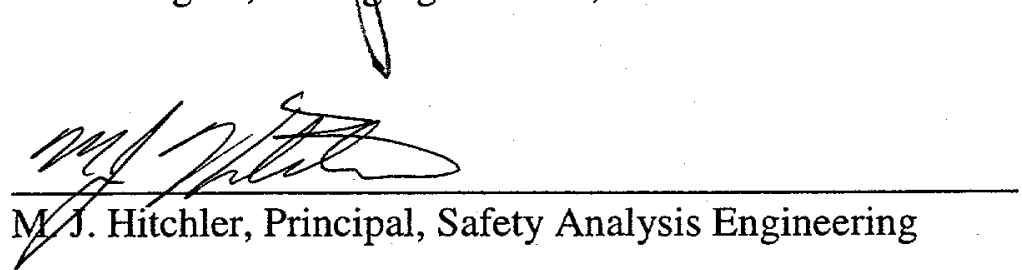

$6 / 9 / 99$ Date $\frac{6 / 9 / 99}{\text { Date }}$ $6 / 23 / 99$ Date

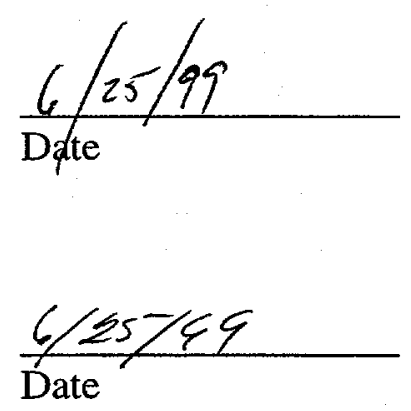




\begin{abstract}
The relocation impacts of a postulated accidental release, scenario 1-RD-3, are evaluated for the Savannah River Technology Center (SRTC). The extent of the area potentially contaminated to a level that would result in doses exceeding the relocation protective action guide (PAG) is calculated. The maximum calculated distance downwind from the accident at which the relocation PAG is exceeded is also determined. The consequences of the particulate portion of the release are evaluated using the HOTSPOT model and an EXCEL spreadsheet. The consequences of the tritium release are evaluated using UFOTRI.
\end{abstract}




\section{CONTENTS}

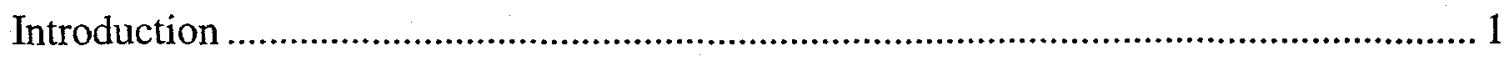

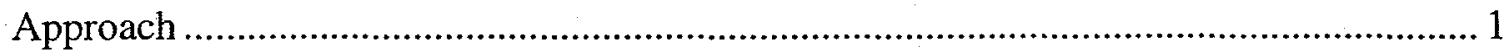

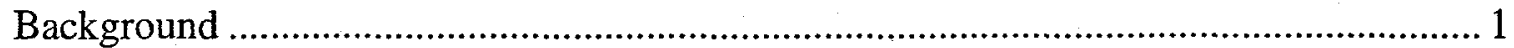

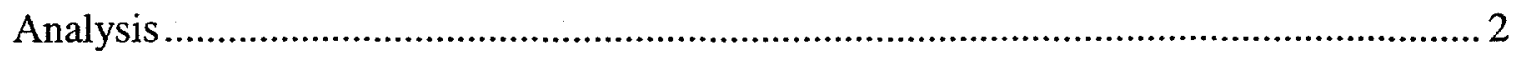

Derivation of the total release .................................................................................. 2

Determination of respirable release .....................................................................

Protective action guides ....................................................................................... 6

Determination of the DRL .................................................................................

Determination of range and area of consequences...................................................... 11

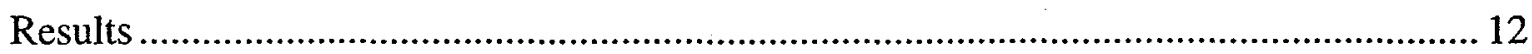

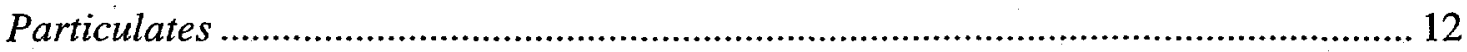

Tritium

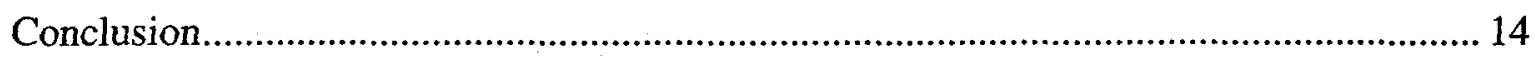

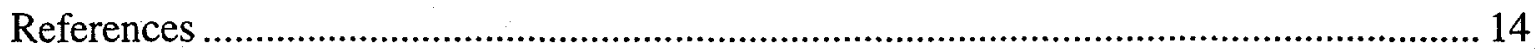

\section{TABLES}

Table 1. Total airborne releases for SRTC scenario 1-RD-3, with mixes and DR not applied

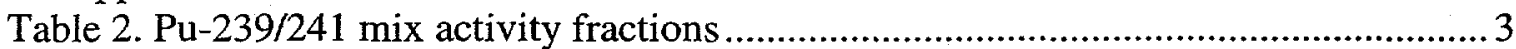

Table 3. Pu-242 mix activity fractions ........................................................................ 4

Table 4. Total airborne releases for SRTC scenario 1-RD-3, unmixed and with DR

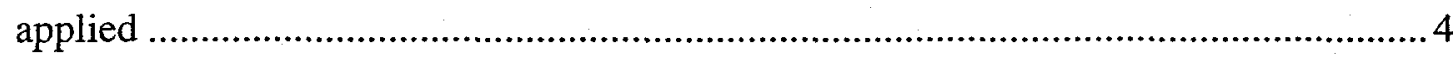

Table 5. Respirable airborne releases for SRTC scenario 1-RD-3, with mixes and DR not

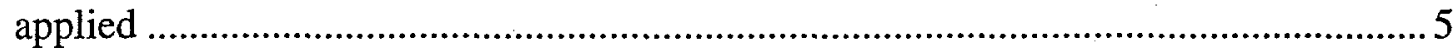

Table 6. Respirable airborne releases for SRTC scenario 1-RD-3, unmixed and DR

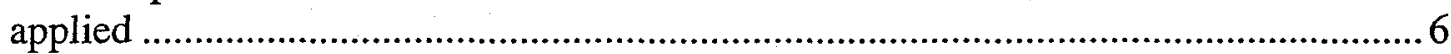

Table 7. PAG values used in this analysis ...................................................................

Table 8. PAGs and DRLs for SRTC particulate release ................................................... 11

Table 9. HOTSPOT input parameters for SRTC release 1-RD-3 ……….......................... 12

Table 10. Additional input parameters for UFOTRI.................................................. 12

Table 11. Ranges (in $\mathrm{km}$ ) until deposition less than relocation DRL, average meteorology, SRTC

Table 12. Areas (in $\mathrm{km}^{2}$ ) with deposition above relocation DRL, average meteorology, SRTC.

Table 13. Ranges (in $\mathrm{km}$ ) until deposition less than relocation DRL, 95\% adverse meteorology, SRTC

Table 14. Areas (in $\mathrm{km}^{2}$ ) with deposition above relocation DRL, 95\% adverse meteorology, SRTC 
Table 15. Dose due to tritium re-emission at $670 \mathrm{~m}$ from the release point .................... 13 


\section{Introduction}

The relocation impacts of a postulated accidental release, scenario 1-RD-3, are evaluated for the Savannah River Technology Center (SRTC). For the particulate release, the size of the area, as well as the maximum distance out to which relocation would be recommended are calculated. For the tritium release, the dose at the site boundary due to re-emission in the first year following the release is calculated and compared to the Protective Action Guide (PAG)

\section{Approach}

The methodology for determining the areas potentially requiring relocation is outlined below.

1. Determine the source terms (radionuclide-specific respirable and total airborne releases).

2. Determine the relocation PAGs for the radionuclides in the source terms in accordance with the Manual of Protective Action Guides and Protective Actions for Nuclear Incidents (EPA 1992).

3. Calculate the doses (from inhalation and ground shine) associated with a unit deposition of (respirable) particulates. The inhalation source term is derived from the respirable airborne release. The ground shine source term is derived from the total (nonrespirable plus respirable) airborne release.

4. Sum the doses resulting from the unit deposition. Divide the relocation PAG by the sum of the doses to obtain the Derived Response Level (DRL) for relocation.

5. Run the dispersion/deposition model HOTSPOT (Homann 1994) to determine areas impacted.

6. If tritium is released, use the UFOTRI model (Raskob 1990 and 1993) to calculate doses from residual tritium in the environment, and compare to PAGs.

\section{Background}

The area affected is considered to be that area in which the deposition due to an accidental release could cause the projected dose to an individual in that area to exceed the PAG. The Environmental Protection Agency (EPA) presents the rationale for its relocation PAG in its Manual of Protective Action Guides and Protective Actions for Nuclear Incidents (EPA 1992, section 4.2.1, p. 4-4 and Appendix E). The objectives of the relocation PAG are to keep the dose in any year after the first year following the accident from exceeding $500 \mathrm{mrem}(5 \mathrm{mSv})$, and to keep the cumulative dose over 50 years (including the first year) from exceeding 5,000 mrem (50 $\mathrm{mSv})$. For the reactor accidents addressed in EPA (1992), the PAG is 2,000 mrem (20 mSv) projected dose ${ }^{*}$. Additionally, it specifies that at 50 times the numerical value of the PAG, relocation to limit beta exposure to skin would be warranted.

\footnotetext{
"The projected sum of effective dose equivalent from external gamma radiation and committed effective dose equivalent from inhalation of resuspended materials, from exposure or intake during the first year.
} 
For fission product releases, relocation decisions are based on whole body irradiation (or effective dose equivalent); preferential irradiation of single tissues is not anticipated to be limiting. However, the radiological hazards associated with many radionuclides (such as plutonium and other actinides) differ significantly from those of the fission product mix considered in EPA (1992). Guidance from the ICRP gives dose limits to single tissues, based on non-stochastic effects that are a factor of ten greater than the effective dose limit based on stochastic effects (ICRP 1977). In general, the radiation associated with the decay of fission products is very penetrating and presents an external hazard (i.e., a person need only be exposed to the radioactive material to receive a dose; the material does not have to be ingested or inhaled) or a whole body irradiation hazard (material inside the body irradiates many tissues as it decays). The radiation associated with the decay of many actinides is not very penetrating and, therefore, only presents a significant radiological hazard when the material is taken into the body, either by inhalation or ingestion. Even then, specific organs are more limiting based on the radiosensitivity of the organ and the distribution of the radionuclides in the body. For the inhalation and ingestion pathways the dose to bone surfaces is more limiting than the effective dose for many actinides.

In order to facilitate comparisons between measured quantities and projected doses, it is often convenient to express a projected dose in terms of a measurable quantity. The value of a measurable quantity that corresponds to a dose value of interest (such as a PAG) is referred to as a derived response level (DRL). Spreadsheet calculations will be performed to determine the relocation DRL for the particulate portion of the nuclide mix of interest. This DRL will then be used in conjunction with the HOTSPOT computer model to determine the size or extent of the area affected by the release of particulates.

The dose resulting from tritium will be evaluated using the UFOTRI model.

\section{Analysis}

\section{Derivation of the total release}

The postulated respirable source terms for SRTC release scenario 1-RD-3 are found in Attachments 2, 3, and 5 of the SRTC Emergency Planning Hazards Assessment (EPHA) (S-EHA-A-00001), Rev. 1, Appendix B, Calculation 1. These respirable source terms are derived from a detailed analysis involving consideration of physical forms and dispersing mechanisms. An overall damage ratio (DR) is applied to the overall source term from the Attachments in S-EHA-A-00001, Rev. 1, Appendix B, Calculation 1 (see sheet 8 of 30 for the DR specification). To determine the total release, it is necessary to "back out" the respirable fraction (RF) from the respirable release source terms. This is done in calculation S-CLC-A-00104 and summarized in Table 1. The activities in Table 1 do not include a correction for a DR less than unity, and the Pu-239/241 and Pu-242 activities are for mixes. 
Table 1. Total airborne releases for SRTC scenario 1-RD-3, with mixes and DR not applied

\begin{tabular}{cc}
\hline Nuclide & $\begin{array}{c}\text { Pre-DR total airborne release } \\
(\mathrm{Ci})\end{array}$ \\
\hline Am-241 & $1.21 \mathrm{E}-02$ \\
$\mathrm{Am}-243$ & $9.68 \mathrm{E}-04$ \\
Ba-133 & $5.38 \mathrm{E}-13$ \\
Ce-144 & $1.90 \mathrm{E}-05$ \\
Cf-249 & $1.27 \mathrm{E}-05$ \\
Cf-252 & $1.59 \mathrm{E}-02$ \\
Cm-244 & $2.03 \mathrm{E}-02$ \\
Cm-246 & $1.00 \mathrm{E}-03$ \\
Co-60 & $7.19 \mathrm{E}-01$ \\
Cs-137 & $1.19 \mathrm{E}+00$ \\
$\mathrm{H}-3$ & $2.14 \mathrm{E}+04$ \\
Np-237 & $4.20 \mathrm{E}-05$ \\
Pm-147 & $2.52 \mathrm{E}-08$ \\
Pu-238 & $5.10 \mathrm{E}-02$ \\
Pu-239 & $1.78 \mathrm{E}-05$ \\
Pu-239/241mix & $4.56 \mathrm{E}-01$ \\
Pu-240 & $9.72 \mathrm{E}-07$ \\
Pu-241 & $2.91 \mathrm{E}-01$ \\
Pu-242mix & $1.28 \mathrm{E}-02$ \\
Sr-90 & $1.18 \mathrm{E}+00$ \\
Tc-99 & $3.60 \mathrm{E}-13$ \\
Th-232 & $1.93 \mathrm{E}-06$ \\
Tl-204 & $9.00 \mathrm{E}-10$ \\
U-235 & $1.78 \mathrm{E}-06$ \\
U-238 & $2.15 \mathrm{E}-04$ \\
\hline
\end{tabular}

Reference: S-CLC-A-00104 Table 1, Sheet 6

Two additional steps are applied to the potential release activities in Table 1. First, the $\mathrm{Pu}-239 / 241$ and $\mathrm{Pu}-242$ mixes must be separated into their constituents. The makeups of these mixes are given in Tables 2 and 3.

Table 2. Pu-239/241 mix activity fractions

\begin{tabular}{cc}
\hline Nuclide & Activity fraction \\
\hline Pu-238 & 0.0073 \\
Pu-239 & 0.2029 \\
Pu-240 & 0.0462 \\
Pu-241 & 0.6761 \\
Pu-242 & 0.0003 \\
Am-241 & 0.0675 \\
\hline
\end{tabular}

Reference: S-CLC-A-00080 Rev. 0, Sheet 4 of 26 
Table 3. Pu-242 mix activity fractions

\begin{tabular}{cc}
\hline Nuclide & Activity fraction \\
\hline $\mathrm{Pu}-238$ & 0.0786 \\
$\mathrm{Pu}-239$ & 0.00024 \\
$\mathrm{Pu}-240$ & 0.01184 \\
$\mathrm{Pu}-241$ & 0.88886 \\
$\mathrm{Pu}-242$ & 0.00074 \\
$\mathrm{Am}-241$ & 0.01977 \\
\hline
\end{tabular}

Reference: S-CLC-A-00080 Rev. 0, Sheet 4 of 26

The final step is to apply a damage ratio (DR) of 0.75 , consistent with the DR assumed for respirable releases in the SRTC EPHA. These total releases are shown in Table 4.

Table 4. Total airborne releases for SRTC scenario 1-RD-3, unmixed and with DR applied

\begin{tabular}{cc}
\hline Nuclide & DR=0.75, unmixed total release (Ci) \\
\hline Am-241 & $3.23 \mathrm{E}-02$ \\
Am-243 & $7.26 \mathrm{E}-04$ \\
Ba-133 & $4.04 \mathrm{E}-13$ \\
Ce-144 & $1.42 \mathrm{E}-05$ \\
Cf-249 & $9.53 \mathrm{E}-06$ \\
Cf-252 & $1.19 \mathrm{E}-02$ \\
Cm-244 & $1.53 \mathrm{E}-02$ \\
Cm-246 & $7.50 \mathrm{E}-04$ \\
Co-60 & $5.40 \mathrm{E}-01$ \\
Cs-137 & $8.91 \mathrm{E}-01$ \\
H-3 & $1.61 \mathrm{E}+04$ \\
Np-237 & $3.15 \mathrm{E}-05$ \\
Pm-147 & $1.89 \mathrm{E}-08$ \\
Pu-238 & $4.15 \mathrm{E}-02$ \\
Pu-239 & $6.94 \mathrm{E}-02$ \\
Pu-240 & $1.59 \mathrm{E}-02$ \\
Pu-241 & $4.58 \mathrm{E}-01$ \\
Pu-242 & $1.10 \mathrm{E}-04$ \\
Sr-90 & $8.84 \mathrm{E}-01$ \\
Tc-99 & $2.70 \mathrm{E}-13$ \\
Th-232 & $1.45 \mathrm{E}-06$ \\
Tl-204 & $6.75 \mathrm{E}-10$ \\
U-235 & $1.34 \mathrm{E}-06$ \\
U-238 & $1.62 \mathrm{E}-04$ \\
\hline
\end{tabular}

Reference: SourceSRTC.xls, Worksheet "Nonresp + resp", columns L to N

Details of these spreadsheet calculations are presented in Attachment A. The total airborne particulate activity released is $2.96 \mathrm{Ci}$. 


\section{Determination of respirable release}

The respirable source terms for SRTC release scenario 1-RD-3 are found in Attachments 2, 3, and 5 of S-EHA-A-00001, Rev. 1, Appendix B, Calculation 1. Respirable source terms from S-EHA-A-00001, Rev. 1, Appendix B, Calculation 1, without a DR of 0.75 and with Pu-239/241 and $\mathrm{Pu}-242$ mixed, are listed in Table 5.

Table 5. Respirable airborne releases for SRTC scenario 1-RD-3, with mixes and DR not applied

\begin{tabular}{cccc}
\hline Nuclide & $\begin{array}{c}\text { Table 5 Pre-DR respirable } \\
\text { airborne release (Ci) }\end{array}$ & $\begin{array}{c}\text { Table } 52 \text { Pre-DR respirable } \\
\text { airborne release (Ci) }\end{array}$ & $\begin{array}{c}\text { Table 71 Pre-DR respirable } \\
\text { airborne release (Ci) }\end{array}$ \\
\hline Am-241 & $2.21 \mathrm{E}-03$ & $1.86 \mathrm{E}-03$ & $5.80 \mathrm{E}-03$ \\
$\mathrm{Am}-243$ & $2.08 \mathrm{E}-04$ & $6.59 \mathrm{E}-05$ & $3.33 \mathrm{E}-04$ \\
$\mathrm{Ba}-133$ & $2.69 \mathrm{E}-13$ & $0.00 \mathrm{E}+00$ & $2.69 \mathrm{E}-13$ \\
$\mathrm{Ce}-144$ & $9.97 \mathrm{E}-07$ & $7.75 \mathrm{E}-06$ & $1.02 \mathrm{E}-05$ \\
$\mathrm{Cf}-249$ & $2.13 \mathrm{E}-06$ & 0 & $8.86 \mathrm{E}-06$ \\
$\mathrm{Cf}-252$ & $7.85 \mathrm{E}-03$ & $1.78 \mathrm{E}-03$ & $6.29 \mathrm{E}-03$ \\
$\mathrm{Cm}-244$ & $6.23 \mathrm{E}-03$ & $3.99 \mathrm{E}-03$ & $1.26 \mathrm{E}-02$ \\
$\mathrm{Cm}-246$ & $3.70 \mathrm{E}-04$ & $2.01 \mathrm{E}-05$ & $2.75 \mathrm{E}-04$ \\
$\mathrm{Co}-60$ & $3.30 \mathrm{E}-01$ & $2.75 \mathrm{E}-02$ & $3.62 \mathrm{E}-01$ \\
$\mathrm{Cs}-137$ & $6.53 \mathrm{E}-02$ & $4.88 \mathrm{E}-01$ & $6.35 \mathrm{E}-01$ \\
$\mathrm{H}-3$ & $1.07 \mathrm{E}+04$ & $9.66 \mathrm{E}+00$ & $1.07 \mathrm{E}+04$ \\
Np-237 & $8.86 \mathrm{E}-06$ & $2.33 \mathrm{E}-08$ & $2.37 \mathrm{E}-05$ \\
Pm-147 & $1.26 \mathrm{E}-08$ & 0 & $1.26 \mathrm{E}-08$ \\
Pu-238 & $1.28 \mathrm{E}-02$ & $1.00 \mathrm{E}-03$ & $9.21 \mathrm{E}-03$ \\
Pu-239 & $8.88 \mathrm{E}-06$ & 0 & $8.88 \mathrm{E}-06$ \\
Pu-239/241mix & $5.96 \mathrm{E}-02$ & $4.21 \mathrm{E}-03$ & $8.09 \mathrm{E}-02$ \\
Pu-240 & $6.85 \mathrm{E}-07$ & 0 & 0 \\
Pu-241 & $4.80 \mathrm{E}-02$ & 0 & $2.03 \mathrm{E}-01$ \\
Pu-242mix & $2.31 \mathrm{E}-03$ & 0 & $7.15 \mathrm{E}-03$ \\
Sr-90 & $6.04 \mathrm{E}-02$ & $4.86 \mathrm{E}-01$ & $6.31 \mathrm{E}-01$ \\
Tc-99 & $1.80 \mathrm{E}-13$ & 0 & $1.80 \mathrm{E}-13$ \\
Th-232 & $1.02 \mathrm{E}-06$ & $7.28 \mathrm{E}-09$ & $8.89 \mathrm{E}-07$ \\
Tl-204 & $4.50 \mathrm{E}-10$ & 0 & $4.50 \mathrm{E}-10$ \\
$\mathrm{U}-235$ & $3.64 \mathrm{E}-07$ & $2.27 \mathrm{E}-08$ & $5.30 \mathrm{E}-07$ \\
$\mathrm{U}-238$ & $2.66 \mathrm{E}-05$ & $2.73 \mathrm{E}-07$ & $4.13 \mathrm{E}-05$ \\
\hline
\end{tabular}

Reference: Source.xls, Worksheets Table 5, Table 52, and Table 71, Column C

Two additional steps are applied to the potential release activities in the preceding table. First, the Pu-239/241 and Pu-242 mixes must be separated into their constituents. The makeups of these mixes are in Tables 2 and 3.

The final step is to apply a damage ratio of 0.75 , consistent with the SRTC EPHA. These respirable releases are shown in Table 6 . The respirable source term for 1-RD-3 is the sum of the three cases, and also presented in Table 6. Details of the calculation are presented in Attachment A. The summed respirable source term in Table 6 is used for subsequent calculations. The total respirable particulate airborne activity, summed over all nuclides and all three cases, is $2.67 \mathrm{Ci}$. 
Table 6. Respirable airborne releases for SRTC scenario 1-RD-3, unmixed and DR applied

\begin{tabular}{ccccc}
\hline Nuclide & $\begin{array}{c}\text { Table 5 DR'd } \\
\text { respirable airborne } \\
\text { release (Ci) }\end{array}$ & $\begin{array}{c}\text { Table 52 DR'd } \\
\text { respirable airborne } \\
\text { release (Ci) }\end{array}$ & $\begin{array}{c}\text { Table 71 DR'd } \\
\text { respirable airborne } \\
\text { release (Ci) }\end{array}$ & $\begin{array}{c}\text { Summed DR'd } \\
\text { respirable airborne } \\
\text { release (Ci) }\end{array}$ \\
\hline Am-241 & $4.71 \mathrm{E}-03$ & $1.61 \mathrm{E}-03$ & $8.55 \mathrm{E}-03$ & $1.49 \mathrm{E}-02$ \\
$\mathrm{Am}-243$ & $1.56 \mathrm{E}-04$ & $4.94 \mathrm{E}-05$ & $2.50 \mathrm{E}-04$ & $4.55 \mathrm{E}-04$ \\
$\mathrm{Ba}-133$ & $2.02 \mathrm{E}-13$ & $0.00 \mathrm{E}+00$ & $2.02 \mathrm{E}-13$ & $4.04 \mathrm{E}-13$ \\
$\mathrm{Ce}-144$ & $7.48 \mathrm{E}-07$ & $5.81 \mathrm{E}-06$ & $7.65 \mathrm{E}-06$ & $1.42 \mathrm{E}-05$ \\
$\mathrm{Cf}-249$ & $1.60 \mathrm{E}-06$ & $0.00 \mathrm{E}+00$ & $6.65 \mathrm{E}-06$ & $8.24 \mathrm{E}-06$ \\
$\mathrm{Cf}-252$ & $5.89 \mathrm{E}-03$ & $1.34 \mathrm{E}-03$ & $4.72 \mathrm{E}-03$ & $1.19 \mathrm{E}-02$ \\
$\mathrm{Cm}-244$ & $4.67 \mathrm{E}-03$ & $2.99 \mathrm{E}-03$ & $9.45 \mathrm{E}-03$ & $1.71 \mathrm{E}-02$ \\
$\mathrm{Cm}-246$ & $2.78 \mathrm{E}-04$ & $1.51 \mathrm{E}-05$ & $2.06 \mathrm{E}-04$ & $4.99 \mathrm{E}-04$ \\
$\mathrm{Co}-60$ & $2.48 \mathrm{E}-01$ & $2.06 \mathrm{E}-02$ & $2.72 \mathrm{E}-01$ & $5.40 \mathrm{E}-01$ \\
$\mathrm{Cs}-137$ & $4.90 \mathrm{E}-02$ & $3.66 \mathrm{E}-01$ & $4.76 \mathrm{E}-01$ & $8.91 \mathrm{E}-01$ \\
$\mathrm{H}-3$ & $8.03 \mathrm{E}+03$ & $7.25 \mathrm{E}+00$ & $8.03 \mathrm{E}+03$ & $1.61 \mathrm{E}+04$ \\
$\mathrm{~Np}-237$ & $6.65 \mathrm{E}-06$ & $1.75 \mathrm{E}-08$ & $1.78 \mathrm{E}-05$ & $2.44 \mathrm{E}-05$ \\
$\mathrm{Pm}-147$ & $9.45 \mathrm{E}-09$ & $0.00 \mathrm{E}+00$ & $9.45 \mathrm{E}-09$ & $1.89 \mathrm{E}-08$ \\
$\mathrm{Pu}-238$ & $9.94 \mathrm{E}-03$ & $7.73 \mathrm{E}-04$ & $7.39 \mathrm{E}-03$ & $1.81 \mathrm{E}-02$ \\
$\mathrm{Pu}-239$ & $9.43 \mathrm{E}-03$ & $6.41 \mathrm{E}-04$ & $1.34 \mathrm{E}-02$ & $2.35 \mathrm{E}-02$ \\
$\mathrm{Pu}-239 / 241 \mathrm{mix}$ & & & & \\
Pu-240 & $2.15 \mathrm{E}-03$ & $1.46 \mathrm{E}-04$ & $3.05 \mathrm{E}-03$ & $5.34 \mathrm{E}-03$ \\
Pu-241 & $6.74 \mathrm{E}-02$ & $2.13 \mathrm{E}-03$ & $1.97 \mathrm{E}-01$ & $2.66 \mathrm{E}-01$ \\
$\mathrm{Pu}-242$ pure & $1.39 \mathrm{E}-05$ & $9.47 \mathrm{E}-07$ & $1.98 \mathrm{E}-05$ & $3.47 \mathrm{E}-05$ \\
Sr-90 & $4.53 \mathrm{E}-02$ & $3.65 \mathrm{E}-01$ & $4.73 \mathrm{E}-01$ & $8.83 \mathrm{E}-01$ \\
Tc-99 & $1.35 \mathrm{E}-13$ & $0.00 \mathrm{E}+00$ & $1.35 \mathrm{E}-13$ & $2.70 \mathrm{E}-13$ \\
Th-232 & $7.65 \mathrm{E}-07$ & $5.46 \mathrm{E}-09$ & $6.67 \mathrm{E}-07$ & $1.44 \mathrm{E}-06$ \\
Tl-204 & $3.38 \mathrm{E}-10$ & $0.00 \mathrm{E}+00$ & $3.38 \mathrm{E}-10$ & $6.75 \mathrm{E}-10$ \\
$\mathrm{U}-235$ & $2.73 \mathrm{E}-07$ & $1.70 \mathrm{E}-08$ & $3.98 \mathrm{E}-07$ & $6.88 \mathrm{E}-07$ \\
$\mathrm{U}-238$ & $2.00 \mathrm{E}-05$ & $2.05 \mathrm{E}-07$ & $3.10 \mathrm{E}-05$ & $5.11 \mathrm{E}-05$ \\
\hline
\end{tabular}

Reference: Source.xls, Worksheet "resp sums", Columns A to F

\section{Protective action guides}

For evaluating the size of the area affected by potential accidents, relocation PAGs were used. Consideration was given to both effective dose and dose to bone surfaces. Since dose limits to single tissues, based on non-stochastic effects, are a factor of ten greater than the effective dose limit based on stochastic effects (ICRP 1977); the limit used for single tissues (e.g., bone surfaces) was ten times that used for effective dose. These PAG values were used for evaluating the size of the area in which relocation may be warranted as a consequence of potential accidents.

For releases consisting primarily of long-lived radionuclides, the relocation PAG level of 2,000 mrem $(20 \mathrm{mSv})$ in the first year may not, by itself, provide the intended level of long-term protection. The relocation PAG is intended to prevent doses exceeding $500 \mathrm{mrem}(5 \mathrm{mSv})$ in any year after the first year following the accident, or $5,000 \mathrm{mrem}(50 \mathrm{mSv})$ over the fifty years immediately following the accident. The relocation PAG values for projected doses that were considered for this work are presented in Table 7. 
Table 7. PAG values used in this analysis

\begin{tabular}{ll}
\hline \multicolumn{1}{c}{ PAG } & \multicolumn{1}{c}{ Timeframe } \\
\hline $\begin{array}{ll}2,000 \mathrm{mrem}(20 \mathrm{mSv}) \text { effective dose, or } 20,000 \mathrm{mrem}(200 \mathrm{mSv}) \\
\text { committed dose equivalent to a single tissue or organ }\end{array}$ & $\begin{array}{l}\text { the first year following an } \\
\text { accident }\end{array}$ \\
$500 \mathrm{mrem}(5 \mathrm{mSv})$ effective dose, or $5,000 \mathrm{mrem}(50 \mathrm{mSv})$ & $\begin{array}{l}\text { any year after the first year } \\
\text { following an accident }\end{array}$ \\
committed dose equivalent to a single tissue or organ & $\begin{array}{l}\text { over } 50 \text { years (including the first } \\
5,000 \mathrm{mrem}(50 \mathrm{mSv}) \text { effective dose, or } 50,000 \mathrm{mrem}(500 \mathrm{mSv})\end{array}$ \\
committed dose equivalent to a single tissue or organ & yeallowing an accident \\
\hline
\end{tabular}

The effective dose is the "projected sum of effective dose equivalent from external gamma radiation and committed effective dose equivalent from inhalation of resuspended materials, from exposure or intake during the" time period of interest (EPA 1992). Similarly, the dose to an individual organ should consider both the committed dose from any intake plus any external contribution to dose, if significant.

\section{Determination of the DRL}

For the general case when the total (respirable plus nonrespirable particulates) release may be different from the respirable release, the following approach is used. Tritium will be treated as a special case.

This analysis is ultimately referenced to a unit deposition of respirable material. The unit respirable deposition has a corresponding deposition of total particulate material. Figure 1 illustrates the steps of this analysis.

The first step is to assume a unit respirable deposition $\left(1 \mu \mathrm{Ci} / \mathrm{m}^{2}\right)$ of the nuclide mix of interest.

This mix is then apportioned to the individual nuclides based on the activity fraction (the ratio of the individual nuclide activity to the sum of the activity released). That is:

$$
\operatorname{dep}_{i}=\operatorname{dep}_{\text {sum }}\left(\frac{A_{i}}{A_{\text {sum }}}\right)
$$

where

$d e p_{i}=$ the respirable deposition concentration of the $\mathrm{i}^{\text {th }}$ nuclide in $\mu \mathrm{Ci} / \mathrm{m}^{2}$, $d e p_{\text {sum }}=$ the sum over all nuclides of the respirable deposition concentration $\left(1 \mu \mathrm{Ci} / \mathrm{m}^{2}\right)$, $A_{i}=$ the respirable activity of the $\mathrm{i}^{\text {th }}$ nuclide (Ci), and $A_{\text {sum }}=$ the sum of the respirable activities released (Ci).

This calculation is performed in Spreadsheet "Source_terms_reloc.xls", Worksheet "Relo_SRTC", Columns E and F. Daughter radionuclide activity fractions are also calculated. Details of the spreadsheet calculations are in Attachment A. 
Respirable Release

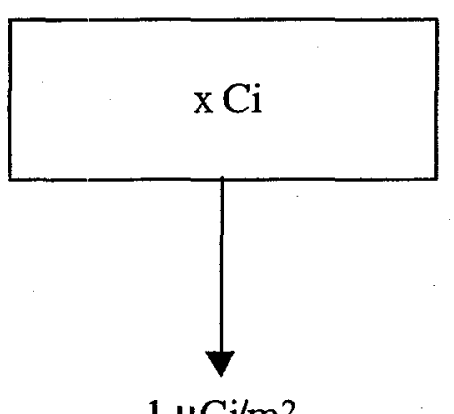

$1 \mu \mathrm{Ci} / \mathrm{m}^{2}$
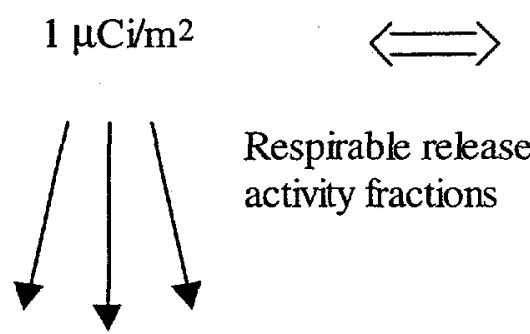

Respirable nuclide-specific deposition $\left(\mu \mathrm{C} / \mathrm{m}^{2}\right)$

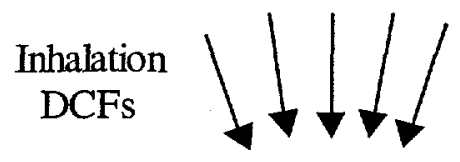

Inhalation dose

$D_{\text {inh, }, \text { unit, resp }}$
Total Release
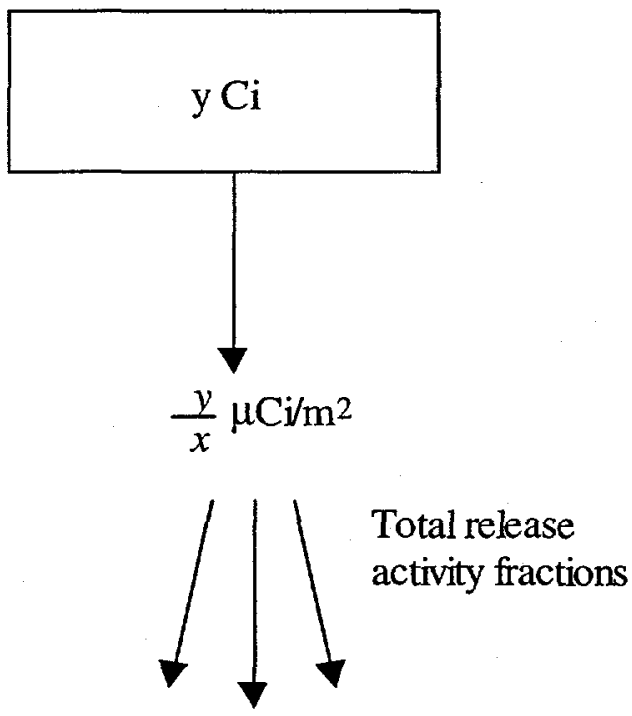

Total (shine) nuclide-specific deposition $\left(\mu \mathrm{Ci} / \mathrm{m}^{2}\right)$

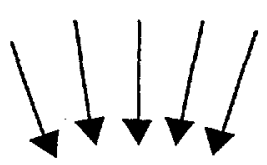

External exposure DCFs
External dose

$D_{\text {ext, unit, resp }}$

$D_{\text {unit }, \text { resp }}=D_{\text {inh, }, \text { unit, }, \text { resp }}+D_{\text {ext, }, \text { unit, resp }}$

Figure 1. Determination of the dose from a unit respirable release

The respirable deposition for each nuclide determined above is used to calculate the dose contributed by that nuclide for inhalation of resuspended material pathways for both effective and organ doses.

The skin dose from beta radiation and external whole-body dose from gamma exposure result from emanations from both respirable and nonrespirable particles that have been deposited on the ground. To find the total (respirable plus nonrespirable) deposition for each radionuclide that corresponds to a unit respirable deposition, two steps are performed. First, total depositions for each nuclide are found for a unit total deposition, using the preceding equation for respirable deposition, except substituting total depositions and activities for respirable depositions and activities. This is performed in Worksheet "Relo_SRTC", Columns C and D. Column D is the activity fraction, which is equivalent to the total deposition of the $\mathrm{i}^{\text {th }}$ nuclide that results from a unit total deposition. 
The postulated respirable release is $x=2.67 \mathrm{Ci}$ (Worksheet "Relo_SRTC", Cell E35) and the total release is $y=2.96 \mathrm{Ci}$ (Worksheet "Relo_SRTC", Cell C35). The assumption is made that nonrespirable particles are deposited at the same rate as respirable particles, so the ratio of total activity deposited per unit area to the respirable activity deposited per unit area, at any location, will be in the same ratio as the total to respirable activity released. A respirable deposition of $1 \mu \mathrm{Ci} / \mathrm{m}^{2}$ corresponds to a total deposition $y / x=2.96 / 2.67=1.11$ times as large, or $1.11 \mu \mathrm{Ci} / \mathrm{m}^{2}$. Multiplication of the total deposition of the $\mathrm{i}^{\text {th }}$ nuclide that results from a unit total deposition by this factor of 1.11 results in the total deposition of the $i^{\text {th }}$ nuclide that results from a unit respirable deposition.

In Worksheet "Relo_SRTC", this $y / x$ term is found in the external EDE (Column I) and skin dose (Column $\mathrm{K}$ ) formulas as \$C \$35/\$E\$35.

Once the respirable and total nuclide-specific depositions are found that correspond to a unit respirable deposition, doses of interest may be found by applying dose conversion factors. Respirable depositions are used to calculate committed dose equivalents to the bone surfaces and committed effective dose equivalents. Total depositions are used to calculate skin doses and doses from external gamma exposure. These calculations are in Columns H, I, K, and L of Worksheet "Relo_SRTC".

The CEDE from a unit respirable deposition is calculated in Column $\mathrm{H}$. The CEDE from inhalation of each radionuclide is summed (Cell H47). The CEDE from each nuclide is the product of the following terms:

$\$ C \$ 7$

$\$ C \$ 3$

$\$ C \$ 4$

$\$ C \$ 5$

$\$ C \$ 6$

$\$ C \$ 8$

$\mathrm{F}(10$ to 34$)$

Relocation!H(10 to 34) unit respirable deposition, $1 \mu \mathrm{Ci} / \mathrm{m}^{2}$

resuspension factor, $\mathrm{m}^{-1}$

breathing rate, $\mathrm{m}^{3} / \mathrm{yr}$

conversion factor, $\mathrm{Bq} / \mu \mathrm{Ci}$

conversion factor, $\mathrm{mrem} / \mathrm{Sv}$

exposure period, 1 yr

respirable activity fraction

CEDE DCF, Sv/Bq

The external dose equivalent from the total deposition corresponding to a unit respirable deposition is calculated in Column I. The external dose from each radionuclide is summed (Cell 147). The external dose equivalent for each nuclide is calculated as the product of the factors below:

$\$ C \$ 7$

$\$ C \$ 8$

$\mathrm{D}(10$ to 45$)$

Relocation!E(10 to 45)

\$C \$35/\$E\$35 unit respirable deposition, $1 \mu \mathrm{Ci} / \mathrm{m}^{2}$

exposure period, $1 \mathrm{yr}$

total activity fraction

external EDE DCF, $\mathrm{mrem} / \mathrm{yr}$ per $\mu \mathrm{Ci} / \mathrm{m}^{2}$

ratio of total deposition to respirable deposition

The skin dose from the total deposition corresponding to a unit respirable deposition is calculated in Column K. The skin dose from each radionuclide is summed (Cell K47). The skin dose for each nuclide is calculated as the product of the factors below: 
$\$ C \$ 7$

$\$ C \$ 8$

$\mathrm{D}(10$ to 45$)$

Relocation! $\mathrm{F}(10$ to 45$)$

\$C\$35/\$E\$35 unit respirable deposition, $1 \mu \mathrm{Ci} / \mathrm{m}^{2}$

exposure period, 1 yr

total activity fraction

skin dose $\mathrm{DCF}, \mathrm{mrem} / \mathrm{yr}$ per $\mu \mathrm{Ci} / \mathrm{m}^{2}$

ratio of total deposition to respirable deposition

The bone surfaces committed dose equivalent (CDE) from a unit respirable deposition is calculated in Column $\mathrm{L}$. The sum of the $\mathrm{CDE}$ from inhalation of each radionuclide is summed (Cell L47). The CDE from each nuclide is the product of the following terms:

$\$ C \$ 7$

$\$ C \$ 3$

$\$ C \$ 4$

$\$ C \$ 5$

$\$ C \$ 6$

$\$ C \$ 8$

$\mathrm{F}(10$ to 34$)$

Relocation!H(10 to 34$)$ unit respirable deposition, $1 \mu \mathrm{Ci} / \mathrm{m}^{2}$

resuspension factor, $\mathrm{m}^{-1}$

breathing rate, $\mathrm{m}^{3} / \mathrm{yr}$

conversion factor, $\mathrm{Bq} / \mu \mathrm{Ci}$

conversion factor, $\mathrm{mrem} / \mathrm{Sv}$

exposure period, 1 yr

respirable activity fraction

bone surfaces $\mathrm{CDE} \mathrm{DCF}, \mathrm{Sv} / \mathrm{Bq}$

Inhalation dose conversion factors (DCFs) are from Federal Guidance Report 11 (EPA 1988). The lung clearance class for each nuclide was chosen to maximize the effective dose. For most nuclides this also maximized the bone surface DCF. External exposure DCFs are from DOE/EH-0070 (1988). To simplify calculations, and due to the anticipated small contribution of the external dose relative to the internal doses, the external contribution to the bone surface dose was approximated by the external contribution to the effective dose. For this nuclide mix and exposure scenario, this approximation did not introduce any appreciable error.

In Figure 1, $D_{\text {unitresp }}$ is the dose due to a unit deposition of respirable material. It includes the CEDE (or the CDE for organ doses) from inhalation of resuspended material (respirable portion of deposited material only) plus the external exposure from ground shine due to the total deposited activity corresponding to the unit respirable deposition.

The calculations just described were for doses due to exposure in the first year following an accident, without considering weathering. They were repeated, taking weathering into consideration, for the three time periods indicated in Table 7 above, with the dose in "any year after the first year following the accident" being represented by the dose in the second year following an accident. Weathering was modeled using the method presented in EPA (1992) where the weathering factor $(W F)$ is defined as

where

$$
W F=\frac{0.63}{\lambda_{1}+\lambda_{2}}\left(1-e^{-\left(\lambda_{1}+\lambda_{2}\right) t}\right)+\frac{0.37}{\lambda_{1}+\lambda_{3}}\left(1-e^{-\left(\lambda_{1}+\lambda_{3}\right) t}\right)
$$

$\mathrm{t}=$ time during which radionuclides are inhaled (or exposure occurs),

$\lambda_{1}=$ radioactive decay constant,

$\lambda_{2}=$ assumed weathering decay constant for 63 percent of the deposited activity, taken as 1.13 per year, and

$\lambda_{3}=$ assumed weathering decay constant for 37 percent of the deposited activity, taken as $7.48 \mathrm{E}-3$ per year. 
To calculate the "weathered" doses for the various time periods, the exposure period referred to in the calculations above was replaced by this weathering factor. The dose due to exposure in the second year was determined by calculating doses due to exposure in the first year and the first two years, then subtracting the one-year dose from the two-year dose.

For a deposition of $1 \mu \mathrm{Ci} \mathrm{m}^{-2}$ of respirable activity of the 1-RD-1.3 mix and a fifty-year exposure period, the inhalation dose is 1,820 mrem CEDE (Worksheet "Relo_SRTC 50yr, Cell H47) and $31,950 \mathrm{mrem}$ CDE to the bone surfaces (Worksheet "Relo_SRTC 50yr, Cell L47). A respirable deposition of $1 \mu \mathrm{Ci} \mathrm{m}^{-2}$ corresponds to a total deposition of $1.11 \mu \mathrm{Ci} \mathrm{m}{ }^{-2}$. A total deposition of $1.11 \mu \mathrm{Ci} \mathrm{m}^{-2}$ gives an external dose of $150 \mathrm{mrem}$ (Worksheet "Relo_SRTC 50yr, Cell I47). Adding the external and internal doses gives 1,970 mrem CEDE and 32,100 mrem CDE to the bone surfaces per deposition of $1 \mu \mathrm{Ci} \mathrm{m}^{-2}$ of respirable activity.

The DRLs may now be determined by dividing the PAGs by their corresponding dose per unit deposition. The DRLs associated with the effective and bone surface doses are much more limiting than those associated with the skin dose and are presented in Table 8. "Bone-surface dose" is the CDE to the bone surfaces. The DRLs calculated using the dose due to fifty years of exposure are the most limiting. Therefore, the DRLs presented for further analysis are those associated with effective and bone surface doses for fifty years of exposure. Details of the spreadsheet calculations are in Attachment A.

Table 8. PAGs and DRLs for SRTC particulate release

\begin{tabular}{cccl}
\hline $\begin{array}{c}\text { PAG } \\
(\mathrm{mrem})\end{array}$ & Description & $\begin{array}{c}\text { DRL } \\
\left(\mu \mathrm{Ci} \mathrm{m} \mathrm{m}^{-2}\right)\end{array}$ & \multicolumn{1}{c}{ DRL Formula } \\
\hline 2000 & Effective dose, first year, no weathering & 9.6 & DRL = PAG/(CEDE+External) \\
20000 & Bone-surface dose, first year, no weathering & 8.2 & DRL = PAG/(BS Dose + External) \\
2000 & Effective dose, first year, with weathering & 14.6 & DRL = PAG/(CEDE+External) \\
20000 & Bone-surface dose, first year, with weathering & 11.2 & DRL = PAG/(BS Dose + External) \\
500 & Effective dose, second year, with weathering & 5.9 & DRL = PAG/(CEDE+External) \\
5000 & Bone-surface dose, second year, with & 4.3 & DRL = PAG/(BS Dose + External) \\
& $\quad$ weathering & & \\
5000 & Effective dose, fifty years, with weathering & 2.5 & DRL $=$ PAG/(CEDE+External) \\
50000 & Bone-surface dose, fifty years, with & 1.6 & DRL $=$ PAG/(BS Dose + External) \\
& $\quad$ weathering & & \\
\hline
\end{tabular}

Reference: Source_terms_reloc.xls, Worksheet "Relo_SRTC DRLs Summary".

Tritium exposures will be evaluated by direct comparisons to the PAG, instead of using a DRL.

\section{Determination of range and area of consequences}

The HOTSPOT computer code was selected to evaluate the consequences of particulate releases (WSRC-TR-98-00392). In the following calculations, a source term equal to the respirable particulate activity of $2.67 \mathrm{Ci}$ (summed over all radionuclides except tritium) is used, and the range and area affected for different meteorological parameters and DRLs found. Pasquill-Briggs dispersion parameters are used. 
HOTSPOT Parameters used for the SRTC release are listed in Table 9. These meteorological data were taken from the SRTC EPHA (S-EHA-A-00001, Rev.1).

Table 9. HOTSPOT input parameters for SRTC release 1-RD-3

\begin{tabular}{ccc}
\hline Parameter & Average Meteorology & $95 \%$ Adverse Meteorology \\
\hline Release height & Ground & Ground \\
Wind speed & $2.5 \mathrm{~m} / \mathrm{s}$ & $1.7 \mathrm{~m} / \mathrm{s}$ \\
Surface roughness & $100 \mathrm{~cm}$ & $100 \mathrm{~cm}$ \\
Stability class & $\mathrm{C}$ & $\mathrm{E}$ \\
Deposition velocity & $0.1 / 1 / 10 \mathrm{~cm} / \mathrm{sec}$ & $0.1 / 1 / 10 \mathrm{~cm} / \mathrm{sec}$ \\
Release duration & $60 \mathrm{~min}$ & $60 \mathrm{~min}$ \\
Inversion layer & $500 \mathrm{~m}$ & $200 \mathrm{~m}$ \\
\hline
\end{tabular}

The tritium release is evaluated using the computer dose model UFOTRI (Raskob 1990, 1993) and the meteorological parameters in Table 9 (particulate deposition velocity is not applicable). Table 10 provides a listing of some of the additional parameters required in UFOTRI.

Table 10. Additional input parameters for UFOTRI

\begin{tabular}{|cl|ll|}
\hline Additional Information & & Meteorology & \\
Chemical Forms & $0 \% \mathrm{HT} / 100 \% \mathrm{HTO}$ & Air temperature & $32.22^{\circ} \mathrm{C}$ \\
No building wake & & No Rainfall & $0 \mathrm{~mm} / \mathrm{h}$ \\
Initial soil water content & $35 \%$ & Thermal energy of release & $0 \mathrm{cal} / \mathrm{sec}$ \\
Relative humidity & 0.8 & Wind direction (in degrees) & 315 \\
& & Dispersion parameters & Briggs \\
\hline Soil and vegetation & & Code coefficients \\
Soil type & Sandy loam & HTO dry deposition velocity* $0.5 \mathrm{~cm} / \mathrm{s}$ \\
Pore volume (default) & $50 \%$ & HT dry deposition velocity* $0.05 \mathrm{~cm} / \mathrm{s}$ \\
\hline
\end{tabular}

* Deposition is calculated in UFOTRI without user-specified constants; values are place holders.

\section{Results}

\section{Particulates}

Tables 11 through 14 summarize the results obtained for SRTC accident scenario 1-RD-3 using the HOTSPOT computer code. The plots from which these data were obtained are included in Attachments B through G. For the average meteorology case, Tables 11 and 12 present the ranges and areas, respectively. "N/E" is used to indicate cases where the area affected was insignificant. Tables 13 and 14 contain results for runs made using adverse meteorological data. 
Table 11. Ranges (in $\mathrm{km}$ ) until deposition less than relocation DRL, average meteorology, SRTC

\begin{tabular}{ccccc}
\hline Basis for DRL & $\mathrm{DRL}$ & \multicolumn{3}{c}{ Deposition Velocity } \\
\cline { 3 - 5 } & $\left(\mu \mathrm{Ci} \mathrm{m}^{-2}\right)$ & $0.1 \mathrm{~cm} \mathrm{~s}-1$ & $1 \mathrm{~cm} \mathrm{~s}-1$ & $10 \mathrm{~cm} \mathrm{~s}-1$ \\
\hline Effective Dose & 2.5 & $<0.02$ & 0.15 & 0.36 \\
Bone Surface Dose & 1.6 & $<0.02$ & 0.18 & 0.44 \\
\hline
\end{tabular}

Table 12. Areas (in $\mathrm{km}^{2}$ ) with deposition above relocation DRL, average meteorology, SRTC

\begin{tabular}{ccccc}
\hline Basis for DRL & DRL & \multicolumn{3}{c}{ Deposition Velocity } \\
\cline { 3 - 5 } & $\left(\mu \mathrm{Ci} \mathrm{m}^{-2}\right)$ & $0.1 \mathrm{~cm} \mathrm{~s}^{-1}$ & $1 \mathrm{~cm} \mathrm{~s}^{-1}$ & $10 \mathrm{~cm} \mathrm{~s}^{-1}$ \\
\hline Effective Dose & 2.5 & $\mathrm{~N} / \mathrm{E}$ & $5.8 \mathrm{E}-03$ & $3.5 \mathrm{E}-02$ \\
Bone Surface Dose & 1.6 & $\mathrm{~N} / \mathrm{E}$ & $8.7 \mathrm{E}-03$ & $5.3 \mathrm{E}-02$ \\
\hline
\end{tabular}

"N/E" indicates the area affected is insignificant.

Table 13. Ranges (in $\mathrm{km}$ ) until deposition less than relocation DRL, 95\% adverse meteorology, SRTC

\begin{tabular}{ccccc}
\hline Basis for DRL & DRL & \multicolumn{3}{c}{ Deposition Velocity } \\
\cline { 3 - 5 } & $\left(\mu \mathrm{Ci} \mathrm{m}^{-2}\right)$ & $0.1 \mathrm{~cm} \mathrm{~s}^{-1}$ & $1 \mathrm{~cm} \mathrm{~s}^{-1}$ & $10 \mathrm{~cm} \mathrm{~s}^{-1}$ \\
\hline Effective Dose & 2.5 & 0.14 & 0.4 & 0.48 \\
Bone Surface Dose & 1.6 & 0.18 & 0.55 & 0.58 \\
\hline
\end{tabular}

Table 14. Areas (in $\mathrm{km}^{2}$ ) with deposition above relocation DRL, 95\% adverse meteorology, SRTC

\begin{tabular}{ccccc}
\hline Basis for DRL & $\mathrm{DRL}$ & \multicolumn{3}{c}{ Deposition Velocity } \\
\cline { 3 - 5 } & $\left(\mu \mathrm{Ci} \mathrm{m}^{-2}\right)$ & $0.1 \mathrm{~cm} \mathrm{~s}^{-1}$ & $1 \mathrm{~cm} \mathrm{~s}^{-1}$ & $10 \mathrm{~cm} \mathrm{~s}^{-1}$ \\
\hline Effective Dose & 2.5 & $2.2 \mathrm{E}-03$ & $2.2 \mathrm{E}-02$ & $3.3 \mathrm{E}-02$ \\
Bone Surface Dose & 1.6 & $3.7 \mathrm{E}-03$ & $3.6 \mathrm{E}-02$ & $4.8 \mathrm{E}-02$ \\
\hline
\end{tabular}

\section{Tritium}

UFOTRI was run to evaluate the dose due to tritium re-emission. The dose due to re-emission (for the first year following the accidental release) was calculated by subtracting the plume passage dose from the dose due to plume passage plus re-emission. These values are presented in Table 15 for the site boundary, a distance of $670 \mathrm{~m}$ from the release point.

Table 15. Dose due to tritium re-emission at $670 \mathrm{~m}$ from the release point

\begin{tabular}{lccc}
\hline & $\begin{array}{c}\text { HTO } \\
\text { plume passage } \\
\text { dose } \\
\text { (mrem) }\end{array}$ & $\begin{array}{c}\text { HTO } \\
\text { plume } \\
\text { passage plus } \\
\text { re-emission } \\
\text { (mrem) }\end{array}$ & $\begin{array}{c}\text { Re-emission } \\
\text { (mrem) }\end{array}$ \\
\hline Average meteorology & 5.12 & 6.43 & 1.3 \\
Adverse meteorology & 38.6 & 50.5 & 12 \\
\hline
\end{tabular}

The dose due to tritium re-emission in the first year following a release is the maximum that would be expected, and that is small compared to the PAGs which govern relocation recommendations. 


\section{Conclusion}

Even though SRTC is near the site boundary (a distance of approximately $0.67 \mathrm{~km}$ ), no relocation protective actions are likely to be recommended for either the particulate release or the tritium release from this scenario.

\section{References}

Department of Energy. External dose-rate conversion factors for calculation of dose to the public. Washington, DC: DOE; DOE/EH-0070, 1988.

Environmental Protection Agency. Limiting values of radionuclide intake and air concentration and dose conversion factors for inhalation, submersion, and ingestion. Washington, DC: EPA; Federal Guidance Report No. 11, EPA 520/1-88-020, 1988.

Environmental. Protection Agency. Manual of protective action guides and protective actions for nuclear incidents. Washington, DC: EPA; EPA 400-R-92-001, 1992.

Hadlock, D. J., SRTC Bounding Accident Source Term and Consequence Assessment for EmergencyPlanning, Aiken, SC: Westinghouse Savannah River Company; S-CLC-A-00080, Rev. 0, 4/14/97.

Hadlock, D. J., Emergency Preparedness Hazards Assessment for the SRTC TA, Aiken, SC: Westinghouse Savannah River Company; S-EHA-A-00001, Rev. 1, September 30, 1997.

International Commission on Radiological Protection. Recommendations of the International Commission on Radiological Protection. Oxford: Pergamon Press; ICRP Publication 26; Ann. ICRP 1(3); 1977.

Raskob, W. UFOTRI: Program for Assessing the Off-Site Consequences from Accidental Tritium Releases. Kernforschungszentrum Karlsruhe, KfK 4605, 1990.

Raskob, W. Description of the New Version 4.0 of the Tritium Model UFOTRI Including User Guide. Kernforschungszentrum Karlsruhe, KfK 5194, August 1993.

Thompson, J. M. Total Airborne Release for SRTC Accident Scenario 1-RD-3 (U), Aiken, SC; Westinghouse Savannah River Company; S-CLC-A-00104, Rev. 0; March 1999. 


\section{Attachments}

Attachment A

Spreadsheet calculations

Attachment B

HOTSPOT results - average meteorology, $0.1 \mathrm{~cm} \mathrm{~s}^{-1}$

Attachment C

HOTSPOT results - average meteorology, $1.0 \mathrm{~cm} \mathrm{~s}^{-1}$

Attachment D

HOTSPOT results - average meteorology, $10 \mathrm{~cm} \mathrm{~s}^{-1}$

Attachment E

HOTSPOT results - adverse meteorology, $0.1 \mathrm{~cm} \mathrm{~s}^{-1}$

Attachment $\mathbf{F}$

HOTSPOT results - adverse meteorology, $1.0 \mathrm{~cm} \mathrm{~s}^{-1}$

Attachment G

HOTSPOT results - adverse meteorology, $10 \mathrm{~cm} \mathrm{~s}^{-1}$ 


\section{Attachment A}

Spreadsheet calculations 


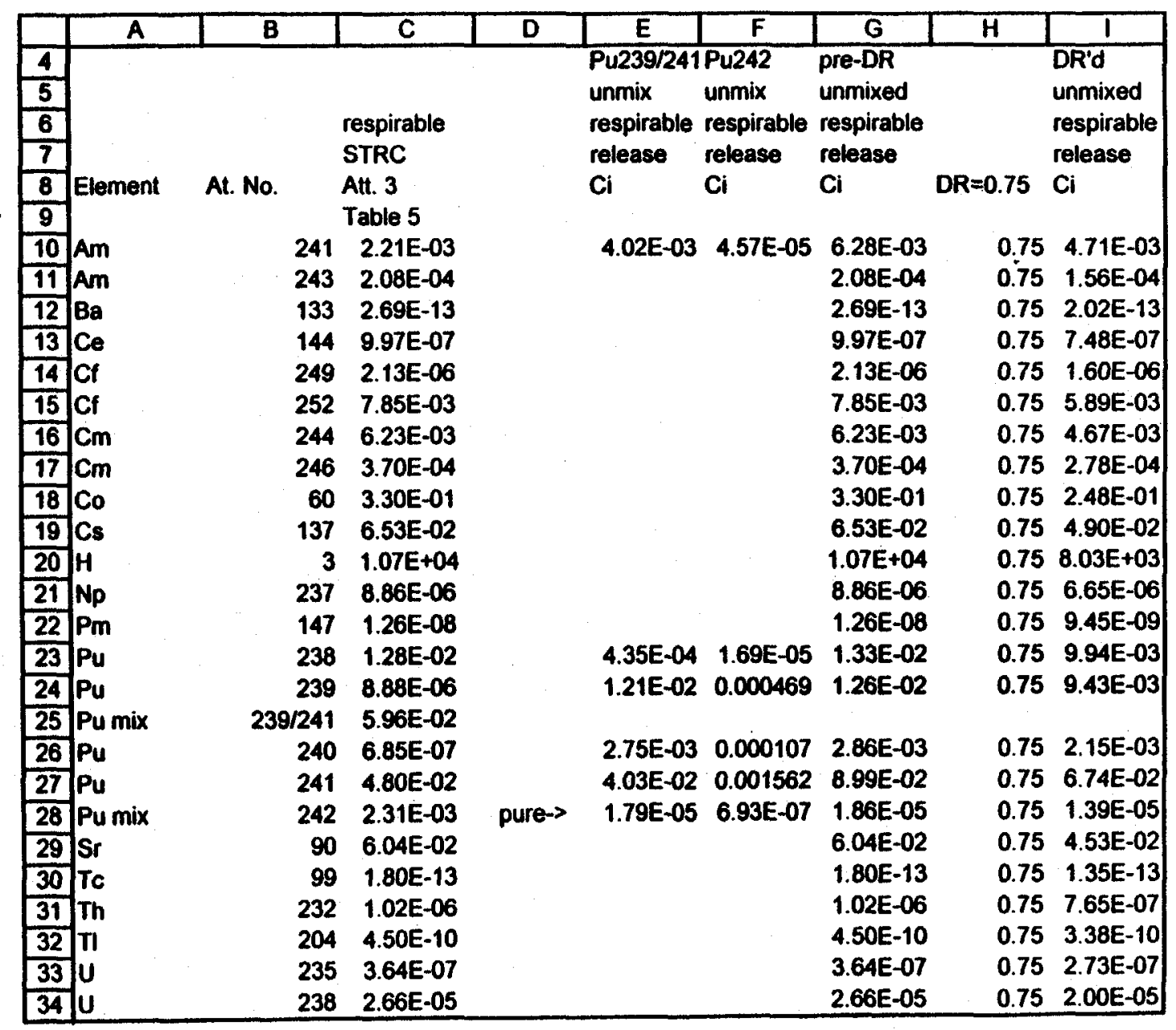




\begin{tabular}{|c|c|c|c|c|c|c|c|c|c|}
\hline & A & $\mathbf{B}$ & C & $\bar{D}$ & $\bar{E}$ & $\mathbf{F}$ & $\overline{\mathbf{G}}$ & $\mathrm{H}$ & I \\
\hline 4 & & & & & Pu239/241 & Pu242 & pre-DR & & DR'd \\
\hline 5 & & & & & unmix & unmix & unmixed & & unmixed \\
\hline 6 & & & respirable & & respirable & respirable & respirable & & respirable \\
\hline 7 & & & STRC & & release & release & release & & release \\
\hline 8 & Element & At. No. & Att 3 & & $\mathrm{Ci}$ & $\mathrm{Ci}$ & $\mathbf{C i}$ & $D R=0.75$ & $\mathrm{Ci}$ \\
\hline 9 & & & Table 5 & & & & & & \\
\hline 10 & Am & 241 & 0.00221 & & $=\operatorname{sc\$ } 25^{\circ} \mathrm{C} 43$ & $=\$ C \$ 28^{*} \mathrm{C} 52$ & $=C 10+E 10+F 10$ & 0.75 & $=\mathrm{G} 10^{*} \mathrm{H} 10$ \\
\hline 11 & Am & 243 & 0.000208 & & & & $=C 11+E 11+F 11$ & 0.75 & $=\mathrm{G} 11 * \mathrm{H} 11$ \\
\hline 12 & $\mathrm{Ba}$ & 133 & 0.000000000000269 & & & & $=C 12+E 12+F 12$ & 0.75 & $=G 12^{*} H 12$ \\
\hline 13 & Ce & 144 & 0.000000997 & & & & $=C 13+E 13+F 13$ & 0.75 & $=\mathrm{G}_{13}{ }^{*} \mathrm{H} 13$ \\
\hline 14 & Cf & 249 & 0.00000213 & & & & $=C 14+E 14+F 14$ & 0.75 & $=\mathrm{G} 14^{*} \mathrm{H} 14$ \\
\hline 15 & $\mathrm{Cf}$ & 252 & 0.00785 & & & & $=C 15+E 15+F 15$ & 0.75 & $=G 15^{*} H 15$ \\
\hline 16 & $\mathrm{Cm}$ & 244 & 0.00623 & & & & $=C 16+E 16+F 16$ & 0.75 & $=\mathrm{G} 16^{\star} \mathrm{H} 16$ \\
\hline 17 & $\mathrm{Cm}$ & 246 & 0.00037 & & & & $=\mathrm{C} 17+\mathrm{E} 17+\mathrm{F} 17$ & 0.75 & $=\mathrm{G} 17^{*} \mathrm{H} 17$ \\
\hline 18 & Co & 60 & 0.33 & & & & $=C 18+E 18+F 18$ & 0.75 & $=G 18 * H 18$ \\
\hline 19 & Cs & 137 & 0.0653 & & & & $=C 19+E 19+F 19$ & 0.75 & $=G 19 * H 19$ \\
\hline 20 & $H$ & 3 & 10700 & & & & $=C 20+E 20+F 20$ & 0.75 & $=G 20^{*} \mathrm{H} 20$ \\
\hline 21 & $\mathrm{~Np}$ & 237 & 0.00000886 & & & & $=C 21+E 21+F 21$ & 0.75 & $=G 21 * H 21$ \\
\hline 22 & $\mathrm{Pm}$ & 147 & 0.0000000126 & & & & $=C 22+E 22+F 22$ & 0.75 & $=G 22^{*} \mathrm{H} 22$ \\
\hline 23 & $\mathrm{Pu}$ & 238 & 0.0128 & & $=\$ \operatorname{co} \$ 25^{*} \operatorname{Cos} 8$ & $=\$ C \$ 28^{\circ} \mathrm{C} 38$ & $=C 23+E 23+F 23$ & 0.75 & $=G 23^{*} \mathrm{H} 23$ \\
\hline 24 & Pu & 239 & 0.00000888 & & $=\$ C \$ 25^{\circ} \operatorname{Cog}$ & $=\$ C \$ 28^{\circ} \mathrm{C} 39$ & $=\mathrm{C} 24+\mathrm{E} 24+\mathrm{F} 24$ & 0.75 & $=G 24^{*} \mathrm{H} 24$ \\
\hline 25 & Pu mix & 23 & 0.0596 & & & & & & \\
\hline 26 & $\mathbf{P u}$ & 240 & 0.000000685 & & $=\$ C \$ 25^{*} \mathrm{C} 40$ & $=\operatorname{ses} 28^{*} \mathrm{C} 40$ & $=C 26+E 26+F 26$ & 0.75 & $=G 26 * \mathrm{H} 26$ \\
\hline 27 & $\mathbf{P u}$ & 241 & 0.048 & & $=\$ C \$ 25^{\circ} \mathrm{C} 41$ & $=\$ C \operatorname{Co} 28^{*} \mathrm{C} 41$ & $=C 27+E 27+F 27$ & 0.75 & $=\mathrm{G} 27^{*} \mathrm{H} 27$ \\
\hline 28 & Pu mix & 242 & 0.00231 & pure-> & $=\$ C \$ 25^{\circ} \mathrm{C} 42$ & $=\$ C \$ 28^{*} \mathrm{C} 42$ & $=E 28+F 28$ & 0.75 & $=G 28^{*} \mathrm{H} 28$ \\
\hline 29 & $\mathrm{Sr}$ & 90 & 0.0604 & & & & $=C 29+E 29+F 29$ & 0.75 & $=G 29 * \mathrm{H} 29$ \\
\hline 30 & $\mathrm{Tc}$ & 99 & 0.00000000000018 & & & & $=C 30+E 30+F 30$ & 0.75 & $=G 30 * H 30$ \\
\hline 31 & Th & 232 & 0.00000102 & & & & $=C 31+E 31+F 31$ & 0.75 & $=G 31 * H 31$ \\
\hline 32 & $\pi$ & 204 & 0.00000000045 & & & & $=C 32+E 32+F 32$ & 0.75 & $=G 32^{*} \mathrm{H} 32$ \\
\hline 33 & $\mathbf{U}$ & 235 & 0.000000364 & & & & $=C 33+E 33+F 33$ & 0.75 & $=G 33^{*} H 33$ \\
\hline 34 & $\mathbf{u}$ & 238 & 0.0000266 & & & & $=C 34+E 34+F 34$ & 0.75 & $=G 34^{*} \mathrm{H} 34$ \\
\hline
\end{tabular}




\begin{tabular}{|c|c|c|c|}
\hline & $\bar{A}$ & $\bar{B}$ & C \\
\hline 36 & \multicolumn{3}{|c|}{ Pu $239 / 241$ mix activity fractions } \\
\hline 37 & \multicolumn{3}{|c|}{ nuclide activity frac } \\
\hline 38 & Pu & 238 & 0.0073 \\
\hline 39 & Pu & 239 & 0.2029 \\
\hline 40 & Pu & 240 & 0.0462 \\
\hline 41 & $\mathbf{P u}$ & 241 & 0.6761 \\
\hline 42 & $\mathrm{Pu}$ & 242 & 0.0003 \\
\hline 43 & Am & 241 & 0.0675 \\
\hline 44 & & & \\
\hline 45 & \multicolumn{3}{|c|}{ Pu 242 mix activity fractions } \\
\hline 46 & nuclide & & ivity frac \\
\hline 47 & $\mathbf{P u}$ & 238 & 0.0786 \\
\hline 48 & Pu & 239 & 0.00024 \\
\hline 49 & Pu & 240 & 0.01184 \\
\hline 50 & Pu & 241 & 0.888886 \\
\hline 51 & $\mathrm{Pu}$ & 242 & 0.00074 \\
\hline 52 & Am & 241 & 0.01977 \\
\hline
\end{tabular}




\begin{tabular}{|c|c|c|c|c|c|c|c|c|c|}
\hline & $\bar{A}$ & $\bar{B}$ & C & $\bar{D}$ & $\mathbf{E}$ & $\bar{F}$ & G & $\bar{H}$ & $T$ \\
\hline 4 & & & & & Pu239/24 & Pu242 & pre-DR & & DR'd \\
\hline 5 & & & & & unmix & unmix & unmixed & & unmixed \\
\hline 6 & & & respirable & & respirable & respirable & respirable & & respirable \\
\hline 7 & & & SRTC & & release & release & release & & release \\
\hline 8 & Element & At. No. & Att. 5 & & $\mathrm{Ci}$ & $\mathrm{Ci}$ & $\mathrm{Ci}$ & $D R=0.75$ & $\mathrm{Ci}$ \\
\hline 9 & & & Table 52 & & & & & & \\
\hline 10 & Am & 241 & $1.86 E-03$ & & $2.84 E-04$ & & $0 \quad 2.14 E-03$ & 0.75 & 1.61E-03 \\
\hline 11 & Am & 243 & 6.59E-05 & & & & 6.59E-05 & $0.75^{\circ}$ & 4.94E-05 \\
\hline 12 & $\mathrm{Ba}$ & 133 & $0.00 E+00$ & & & & $0.00 E+00$ & 0.75 & $0.00 E+00$ \\
\hline 13 & $\mathrm{Ce}$ & 144 & 7.75E-06 & & & & 7.75E-06 & 0.75 & 5.81E-06 \\
\hline 14 & Cf & 249 & 0 & & & & $0.00 E+00$ & 0.75 & $0.00 E+00$ \\
\hline 15 & $\mathrm{Cr}$ & 252 & 1.78E-03 & & & & $1.78 \mathrm{E}-03$ & 0.75 & $1.34 E-03$ \\
\hline 16 & $\mathrm{Cm}$ & 244 & $3.99 E-03$ & & & & $3.99 E-03$ & 0.75 & 2.99E-03 \\
\hline 17 & $\mathrm{Cm}$ & 246 & 2.01E-05 & & & & 2.01E-05 & 0.75 & 1.51E-05 \\
\hline 18 & Co & 60 & $2.75 E-02$ & & & & 2.75E-02 & 0.75 & $2.06 E-02$ \\
\hline 19 & Cs & 137 & 4.88E-01 & & & & 4.88E-01 & 0.75 & 3.66E-01 \\
\hline 20 & H & 3 & $9.66 \mathrm{E}+00$ & & & & $9.66 E+00$ & 0.75 & $7.25 E+00$ \\
\hline 21 & Np & 237 & 2.33E-08 & & & & 2.33E-08 & 0.75 & $1.75 E-08$ \\
\hline 22 & Pm & 147 & 0 & & & & $0.00 E+00$ & 0.75 & $0.00 E+00$ \\
\hline 23 & $\mathrm{Pu}$ & 238 & $1.00 E-03$ & & 3.07E-05 & & $0 \quad 1.03 E-03$ & 0.75 & 7.73E-04 \\
\hline 24 & Pu & 239 & 0 & & 8.54E-04 & & $0 \quad 8.54 E-04$ & 0.75 & 6.41E-04 \\
\hline 25 & Pu mix & $239 / 241$ & 4.21E-03 & & & & & & \\
\hline 26 & Pu & 240 & 0 & & $1.95 E-04$ & & $0 \quad 1.95 E-04$ & 0.75 & $1.46 E-04$ \\
\hline 27 & $\mathbf{P u}$ & 241 & 0 & & 2.85E-03 & & $0 \quad 2.85 E-03$ & 0.75 & 2.13E-03 \\
\hline 28 & Pu mix & 242 & 0 & pure-> & $1.26 E-06$ & & $0 \quad 1.26 E-06$ & 0.75 & 9.47E-07 \\
\hline 29 & Sr & 90 & 4.86E-01 & & & & 4.86E-01 & 0.75 & 3.65E-01 \\
\hline 30 & Tc & 99 & 0 & & & & $0.00 E+00$ & 0.75 & $0.00 E+00$ \\
\hline 31 & Th & 232 & 7.28E-09 & & & & 7.28E-09 & 0.75 & 5.46E-09 \\
\hline 32 & $\mathrm{TI}$ & 204 & 0 & & & & $0.00 E+00$ & 0.75 & $0.00 E+00$ \\
\hline 33 & $\mathbf{u}$ & 235 & 2.27E-08 & & & & 2.27E-08 & 0.75 & 1.70E-08 \\
\hline 34 & $\mathbf{u}$ & 238 & 2.73E-07 & & & & 2.73E-07 & 0.75 & 2.05E-07 \\
\hline
\end{tabular}




\begin{tabular}{|c|c|c|c|c|c|c|c|c|c|}
\hline & A & $\mathbf{B}$ & C & D & $E$ & $F$ & $\mathbf{G}$ & $\mathrm{H}$ & 1 \\
\hline 4 & & & & & Pu239/241 & Pu242 & pre-DR & & DR'd \\
\hline 5 & & & & & unmix & unmix & unmixed & & unmixed \\
\hline 6 & & & respirable & & respirable & respirable & respirable & & respirable \\
\hline 7 & & & SRTC & & release & release & release & & release \\
\hline 8 & Element & At. No. & Att. 5 & & $\mathbf{C i}$ & $\mathrm{Ci}$ & $\mathbf{C i}$ & $\mathrm{DR}=0.75$ & $\mathrm{Ci}$ \\
\hline 9 & & & Table 52 & & & & & & \\
\hline 10 & Am & 241 & 0.00186 & & $=\$ C \$ 25^{*} \mathrm{C} 43$ & $=\$ C \$ 28^{\star} \mathrm{C} 52$ & $=C 10+E 10+F 10$ & 0.75 & $=\mathrm{G} 10^{*} \mathrm{H} 10$ \\
\hline 11 & Am & 243 & 0.0000659 & & & & $=C 11+E 11+F 11$ & 0.75 & $=\mathrm{G} 11^{\circ} \mathrm{H} 11$ \\
\hline 12 & $\mathbf{B a}$ & 133 & 0 & & & & $=C_{12}+E_{12}+F 12$ & 0.75 & $=G 12^{*} \mathrm{H} 12$ \\
\hline 13 & $\mathrm{Ce}$ & 144 & 0.00000775 & & & & $=C 13+E 13+F 13$ & 0.75 & $=G 13^{\prime \prime} H 13$ \\
\hline 14 & Cf & 249 & 0 & & & & $=C 14+E 14+F 14$ & 0.75 & $=G 14^{*} H 14$ \\
\hline 15 & Cf & 252 & 0.00178 & & & & $=C 15+E 15+F 15$ & 0.75 & $=\mathrm{G} 15^{*} \mathrm{H} 15$ \\
\hline 16 & $\mathrm{Cm}$ & 244 & 0.00399 & & & & $=C 16+E 16+F 16$ & 0.75 & $=G 16^{*} \mathrm{H} 16$ \\
\hline 17 & $\mathrm{Cm}$ & 246 & 0.0000201 & & & & $=C 17+E 17+F 17$ & 0.75 & $=G 17^{*} \mathrm{H} 17$ \\
\hline 18 & Co & 60 & 0.0275 & & & & $=C 18+E 18+F 18$ & 0.75 & $=G 18^{*} H 18$ \\
\hline 19 & Cs & 137 & 0.488 & & & & $=C 19+E 19+F 19$ & 0.75 & $=G 19^{\circ} \mathrm{H} 19$ \\
\hline 20 & $\mathrm{H}$ & 3 & 9.66 & & & & $=C 20+E 20+F 20$ & 0.75 & $=G 20^{*} \mathrm{H} 2 \mathrm{O}$ \\
\hline 21 & $\mathrm{~Np}$ & 237 & 0.0000000233 & & & & $=C 21+E 21+F 21$ & 0.75 & $=\mathrm{G} 21 * \mathrm{H} 21$ \\
\hline 22 & $\mathrm{Pm}$ & 147 & 0 & & & & $=C 22+E 22+F 22$ & 0.75 & $=G 22^{*} H 22$ \\
\hline 23 & $\mathbf{P u}$ & 238 & 0.001 & & $=\$ C \$ 25^{\circ} \mathrm{C} 38$ & $=\$ C \$ 28^{*} \mathrm{C} 38$ & $=C 23+E 23+F 23$ & 0.75 & $=\mathrm{G}^{\circ} 3^{\circ} \mathrm{H} 23$ \\
\hline 24 & Pu & 239 & 0 & & $=\$ \operatorname{Cos} 25^{\circ} \mathrm{C} 39$ & $=\$ C \$ 28^{*} C 39$ & $=\mathrm{C} 24+\mathrm{E} 24+\mathrm{F} 24$ & 0.75 & $=G 24^{*} H 24$ \\
\hline 25 & Pu mix & 239 & 0.00421 & & & & & & \\
\hline 26 & $\mathbf{P u}$ & 240 & 0 & & $=\$ C \$ 25^{\star} C 40$ & $=\operatorname{se} \$ 28^{*} \mathrm{C} 40$ & $=C 26+E 26+F 26$ & 0.75 & $=G 26 * \mathrm{H} 26$ \\
\hline 27 & $\mathrm{Pu}$ & 241 & 0 & & $=\$ \operatorname{Cs} 225^{*} C 41$ & $=\$ C S 28^{\circ} C 41$ & $=\mathrm{C} 27+\mathrm{E} 27+\mathrm{F} 27$ & 0.75 & $=G 27 * \mathrm{H} 27$ \\
\hline 28 & Pu mix & 242 & 0 & pure-> & $=\$ C \operatorname{Cs} 25^{\circ} \mathrm{C} 42$ & $=\$ C \$ 28^{\circ} \mathrm{C} 42$ & $=E 28+F 28$ & 0.75 & $=G 28^{*} H 28$ \\
\hline 29 & Sr & 90 & 0.486 & & & & $=C 29+E 29+F 29$ & 0.75 & $=G 29 * H 29$ \\
\hline 30 & Tc & 99 & 0 & & & & $=C 30+E 30+F 30$ & 0.75 & $=G 30^{*} H 30$ \\
\hline 31 & Th & 232 & 0.00000000728 & & & & $=C 31+E 31+F 31$ & 0.75 & $=G 31 * H 31$ \\
\hline 32 & $\mathrm{TI}$ & 204 & 0 & & & & $=C 32+E 32+F 32$ & 0.75 & $=G 32^{*} H 32$ \\
\hline 33 & U & 235 & 0.0000000227 & & & & $=C 33+E 33+F 33$ & 0.75 & $=G 33^{*} H 33$ \\
\hline 34 & $\mathbf{u}$ & 238 & 0.000000273 & & & & $=C 34+E 34+F 34$ & 0.75 & $=G 34^{*} H 34$ \\
\hline
\end{tabular}




\begin{tabular}{|c|c|c|c|}
\hline & $\bar{A}$ & B & \\
\hline 36 & \multicolumn{3}{|c|}{ Pu $239 / 241$ mix activity fractions } \\
\hline 37 & \multicolumn{3}{|c|}{ nuclide $\quad$ activity frac } \\
\hline 38 & $\mathrm{Pu}$ & 238 & 0.0073 \\
\hline 39 & $\mathrm{Pu}$ & 239 & 0.2029 \\
\hline 40 & Pu & 240 & 0.0462 \\
\hline 41 & Pu & 241 & 0.6761 \\
\hline 42 & $\mathrm{Pu}$ & 242 & 0.0003 \\
\hline 43 & Am & 241 & 0.0675 \\
\hline 44 & & & \\
\hline 45 & \multicolumn{3}{|c|}{ Pu 242 mix activity fractions } \\
\hline 46 & nuclid & & \\
\hline 47 & $\mathrm{Pu}$ & 238 & 0.0786 \\
\hline 48 & Pu & 239 & 0.00024 \\
\hline 49 & Pu & 240 & 0.01184 \\
\hline 50 & Pu & 241 & 0.88886 \\
\hline 51 & Pu & 242 & 0.00074 \\
\hline 52 & Am & 241 & 0.01977 \\
\hline
\end{tabular}




\begin{tabular}{|c|c|c|c|c|c|c|c|c|c|}
\hline & A & $\mathbf{B}$ & $\mathbf{C}$ & $\bar{D}$ & $\bar{E}$ & $F$ & $\mathbf{G}$ & $\bar{H}$ & $T$ \\
\hline 4 & \multirow{6}{*}{ Elamen } & \multirow{6}{*}{ At. No. } & \multirow[b]{3}{*}{ respirable } & & \multicolumn{2}{|c|}{ Pu239/241Pu242 } & \multicolumn{2}{|l|}{ pre-DR } & DR'd \\
\hline 5 & & & & & unmix & unmix & unmixed & & unmixed \\
\hline 6 & & & & & respirable & respirable & respirable & & respirable \\
\hline 7 & & & STRC & & release & release & release & & release \\
\hline 8 & & & Att. 2 & & $\mathrm{Ci}$ & $\mathbf{C i}$ & $\mathbf{C i}$ & $\mathrm{DR}=0.75$ & $\mathrm{Ci}$ \\
\hline 8 & & & Table 71 & & & & & & \\
\hline 10 & Am & 241 & 5.80E-03 & & $5.46 E-03$ & 0.000141 & 1.14E-02 & 0.75 & $.8 .55 E-03$ \\
\hline 11 & Am & 243 & 3.33E-04 & & & & 3.33E-04 & 0.75 & 2.50E-04 \\
\hline 12 & $\mathrm{Ba}$ & 133 & $2.69 E-13$ & & & & 2.69E-13 & 0.75 & $2.02 E-13$ \\
\hline 13 & Ce & 144 & $1.02 E-05$ & & & & 1.02E-05 & 0.75 & 7.65E-06 \\
\hline 14 & Cf & 249 & 8.86E-06 & & & & $8.86 E-06$ & 0.75 & $6.65 E-06$ \\
\hline 15 & Cf & 252 & $6.29 E-03$ & & & & $6.29 E-03$ & 0.75 & 4.72E-03 \\
\hline 16 & $\mathrm{Cm}$ & 244 & $1.26 E-02$ & & & & $1.26 E-02$ & 0.75 & $9.45 E-03$ \\
\hline 17 & $\mathrm{Cm}$ & 246 & $2.75 E-04$ & & & & $2.75 E-04$ & 0.75 & 2.06E-04 \\
\hline 18 & Co & 60 & $3.62 E-01$ & & & & 3.62E-01 & 0.75 & $2.72 E-01$ \\
\hline 19 & Cs & 137 & 6.35E-01 & & & & 6.35E-01 & 0.75 & 4.76E-01 \\
\hline 20 & $\mathrm{H}$ & 3 & $1.07 E+04$ & & & & 1.07E+04 & 0.75 & 8.03E+03 \\
\hline 21 & $\mathrm{~Np}$ & 237 & 2.37E-05 & & & & 2.37E-05 & 0.75 & $1.78 E-05$ \\
\hline 22 & Pm & 147 & $1.26 E-08$ & & & & $1.26 E-08$ & 0.75 & $9.45 E-09$ \\
\hline 23 & $\mathrm{Pu}$ & 238 & $9.21 E-03$ & & 5.91E-04 & 5.22E-05 & $9.85 E-03$ & 0.75 & 7.39E-03 \\
\hline 24 & $\mathrm{Pu}$ & 239 & $8.88 E-06$ & & 1.64E-02 & 0.001451 & 1.79E-02 & 0.75 & $1.34 E-02$ \\
\hline 25 & Pu mix & $239 / 241$ & 8.09E-02 & & & & & & \\
\hline 26 & $\mathrm{Pu}$ & 240 & 0 & & $3.74 E-03$ & 0.00033 & 4.07E-03 & 0.75 & $3.05 E-03$ \\
\hline 27 & $\mathbf{P u}$ & 241 & 2.03E-01 & & 5.47E-02 & 0.004834 & 2.63E-01 & 0.75 & 1.97E-01 \\
\hline 28 & Pu mix & 242 & $7.15 E-03$ & pure-> & 2.43E-05 & $2.15 E-06$ & 2.64E-05 & 0.75 & $1.98 E-05$ \\
\hline 29 & $\mathrm{Sr}$ & 90 & 6.31E-01 & & & & 6.31E-01 & 0.75 & 4.73E-01 \\
\hline 30 & TC & 99 & $1.80 E-13$ & & & & 1. $80 \mathrm{E}-13$ & 0.75 & 1.35E-13 \\
\hline 31 & Th & 232 & 8.89E-07 & & & & 8.89E-07 & 0.75 & 6.67E-07 \\
\hline 32 & $\pi$ & 204 & $4.50 E-10$ & & & & $4.50 E-10$ & 0.75 & $3.38 E-10$ \\
\hline 33 & $u$ & 235 & $5.30 E-07$ & & & & 5.30E-07 & 0.75 & 3.98E-07 \\
\hline 34 & $u$ & 238 & 4.13E-05 & & & & 4.13E-05 & 0.75 & 3.10E-05 \\
\hline
\end{tabular}




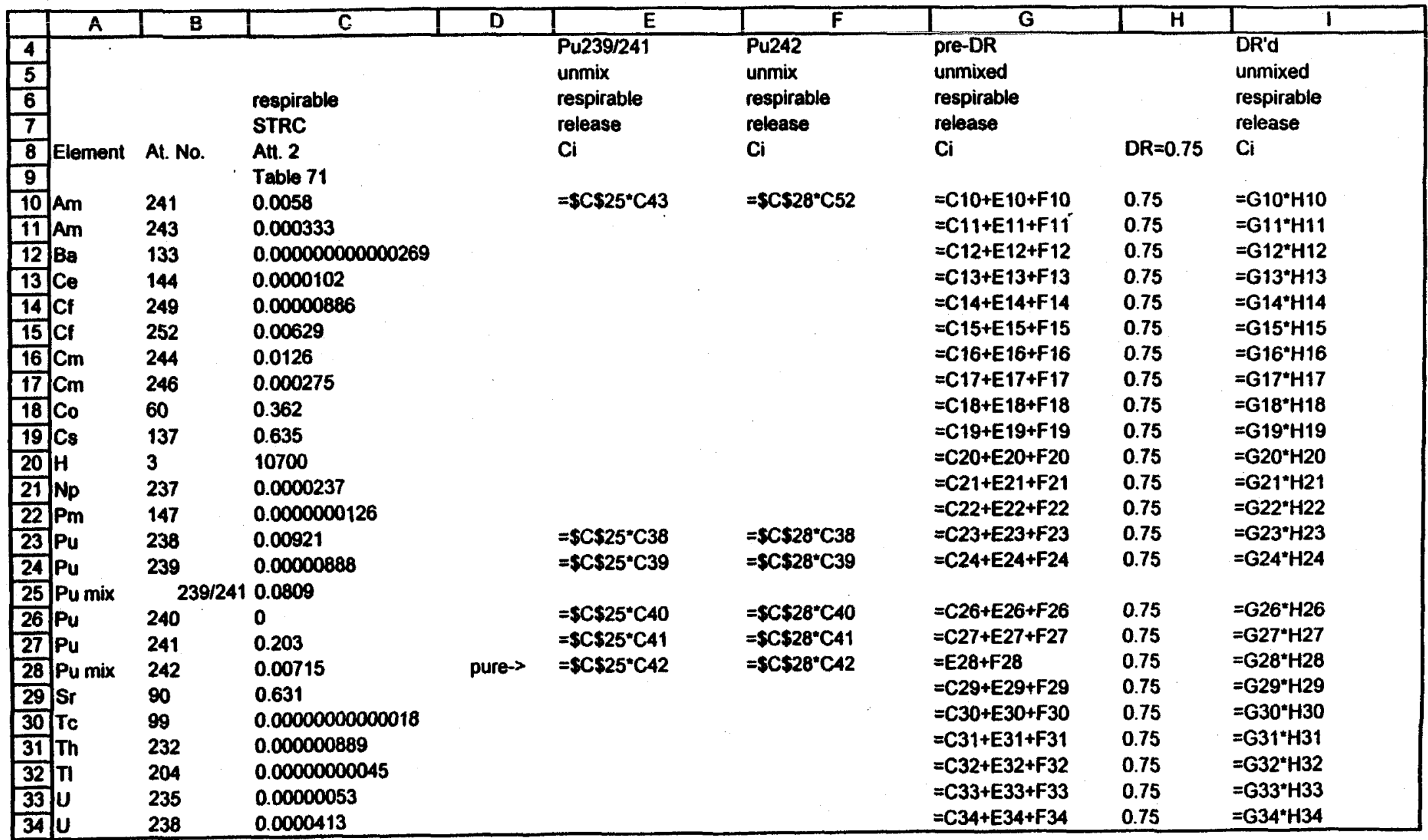




\begin{tabular}{|c|c|c|c|}
\hline & $\bar{A}$ & B & $\mathrm{C}$ \\
\hline 36 & \multirow{2}{*}{\multicolumn{2}{|c|}{$\begin{array}{l}\text { Pu } 239 / 241 \text { mix activity fractions } \\
\text { nuclide }\end{array}$}} & \\
\hline 37 & & & activity frac \\
\hline 38 & $\mathrm{Pu}$ & 238 & 0.0073 \\
\hline 39 & $\mathrm{Pu}$ & 239 & 0.2029 \\
\hline 40 & Pu & 240 & 0.0462 \\
\hline 41 & $\mathrm{Pu}$ & 241 & 0.6761 \\
\hline$\overline{42}$ & $\mathrm{Pu}$ & 242 & 0.0003 \\
\hline 43 & Am & 241 & 0.0675 \\
\hline 44 & \multirow{3}{*}{\multicolumn{2}{|c|}{$\begin{array}{l}\text { Pu } 242 \text { mix activity fractions } \\
\text { nuclide }\end{array}$}} & \\
\hline 45 & & & \\
\hline 46 & & & activity frac \\
\hline 47 & Pu & 238 & 0.0786 \\
\hline 48 & Pu & 239 & 0.00024 \\
\hline 49 & Pu & 240 & 0.01184 \\
\hline 50 & $\mathrm{Pu}$ & 241 & 0.88886 \\
\hline 51 & $\mathrm{Pu}$ & 242 & 0.00074 \\
\hline 52 & Am & 241 & 0.01977 \\
\hline
\end{tabular}




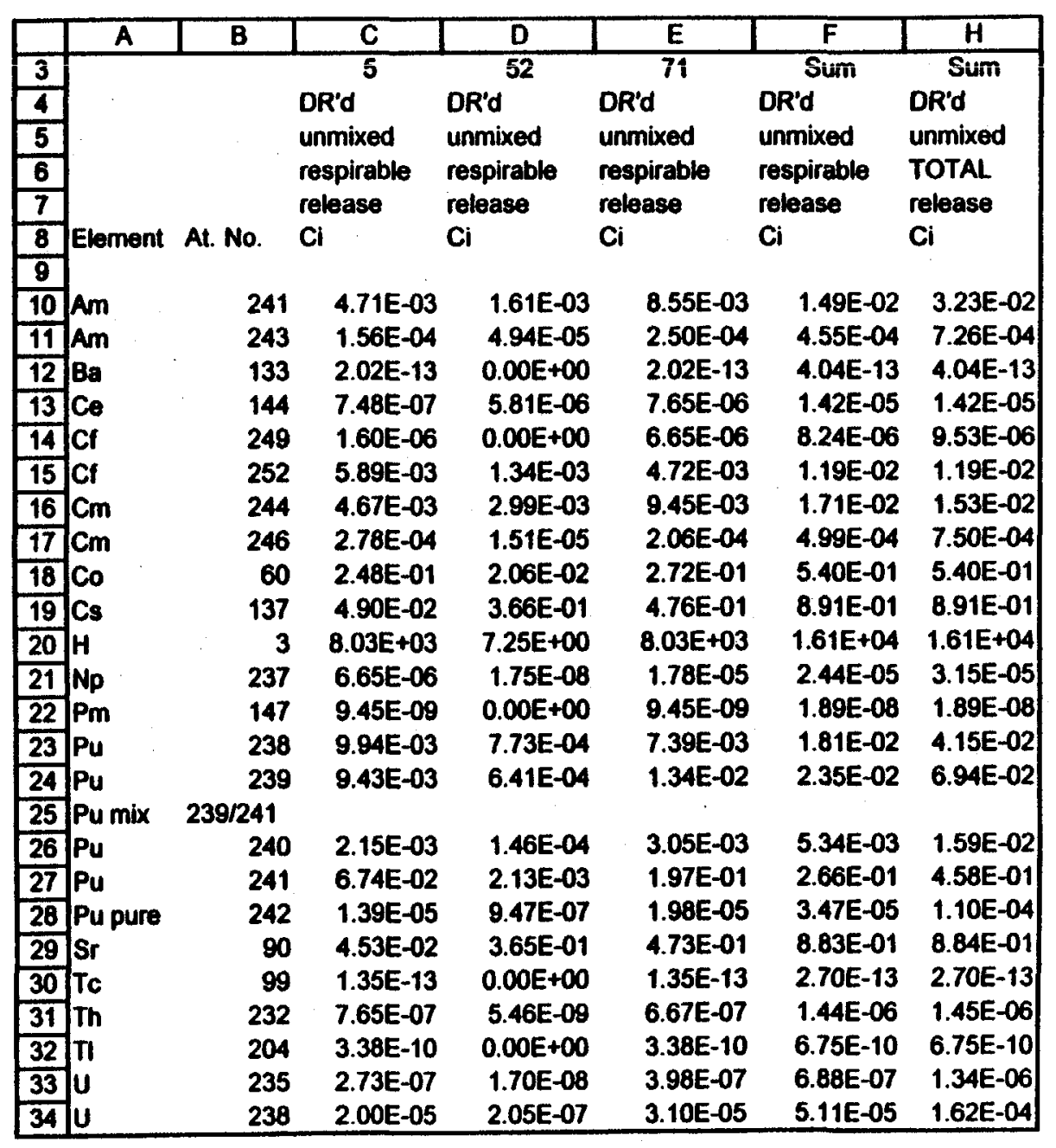




\begin{tabular}{|c|c|c|c|c|c|c|c|}
\hline & $\bar{A}$ & $\mathbf{B}$ & $\mathbf{C}$ & D & $\bar{E}$ & $\mathbf{F}$ & $\mathrm{H}$ \\
\hline 3 & & & 5 & 52 & 71 & Sum & Sum \\
\hline 4 & & & DR'd & DR'd & DR'd & DR'd & DR'd \\
\hline 5 & & & unmixed & unmixed & unmixed & unmixed & unmixed \\
\hline 6 & & & respirable & respirable & respirable & respirable & TOTAL \\
\hline 7 & & & release & release & release & release & release \\
\hline 8 & Element & At. No. & $\mathbf{C i}$ & $\mathrm{Ci}$ & $\mathrm{Ci}$ & $\mathbf{C i}$ & $\mathrm{Ci}$ \\
\hline 9 & & & & & & & \\
\hline 10 & Am & 241 & $=$ Table5॥10 & $={ }^{\prime}$ Table $52 ' \| 10$ & $=$ Table711110 & $=C 10+D 10+E 10$ & $=$ 'Nonresp + resp'IN10 \\
\hline 11 & Am & 243 & $=$ Tablesll11 & $={ }^{\circ}$ Table $52^{\prime} \| 11$ & $=$ Table711l11 & $=C 11+D 11+E 11$ & $=$ 'Nonresp + resp'IN11 \\
\hline 12 & $\mathrm{Ba}$ & 133 & $=$ Table5.112 & $=$ Table $52^{\prime} \| 12$ & $=$ Table71!l12 & $=C 12+D 12+E 12$ & $={ }^{\prime}$ Nonresp + resp'IN12 \\
\hline 13 & $\mathrm{Ce}$ & 144 & $=$ Table5:113 & $=$ 'Table $52^{\prime} ! 113$ & $=$ Table71!l13 & $=C 13+D 13+E 13$ & $=$ 'Nonresp + resp'!N13 \\
\hline 14 & cf & 249 & $=$ Table5!l14 & $={ }^{\prime}$ Table $52^{\prime} \| 14$ & $=$ Table71II14 & $=C 14+D 14+E 14$ & $=$ 'Nonresp + resp'IN 14 \\
\hline 15 & cf & 252 & =Table5II15 & $={ }^{\prime}$ Table $52^{\prime} \| 15$ & $=$ Table71!l15 & $=C 15+D 15+E 15$ & $={ }^{\prime}$ Nonresp + resp'!N15 \\
\hline 16 & $\mathrm{Cm}$ & 244 & $=$ Table5II16 & $=$ 'Table 52 'll16 & $=$ Table711l16 & $=C 16+D 16+E 16$ & $={ }^{\prime}$ Nonresp + resp'IN16 \\
\hline 17 & $\mathrm{Cm}$ & 246 & $=$ Tables 1117 & $=$ Table 52 'll17 & $=$ Table711117 & $=C 17+D 17+E 17$ & $={ }^{\prime}$ Nonresp + resp'iN17 \\
\hline 18 & Co & 60 & $=$ Table51118 & $={ }^{\prime}$ Table $52^{\prime \prime 11}$ & $=$ Table711118 & $=C 18+D 18+E 18$ & $={ }^{\prime}$ Nonresp + resp'!N18 \\
\hline 19 & $\mathrm{Cs}$ & 137 & $=$ Table51119 & $={ }^{\prime}$ Table $52^{\prime} 1119$ & $=$ Table711119 & $=C 19+D 19+E 19$ & 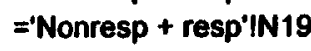 \\
\hline 20 & $H$ & 3 & $=$ Table51120 & $=$ 'Table 52'1120 & $=$ Table71!120 & $=C 20+D 20+E 20$ & $={ }^{\prime}$ Nonresp + resp'IN20 \\
\hline 21 & Np & 237 & $=$ Table5.121 & $={ }^{\circ}$ Table $52^{\prime} \mid 121$ & $=$ Table71!121 & $=C 21+D 21+E 21$ & $={ }^{\prime}$ Nonresp + resp'!N21 \\
\hline 22 & $\mathrm{Pm}$ & 147 & $=$ Tables 1122 & $={ }^{\prime}$ Table $52^{\prime} 1122$ & $=$ Table711122 & $=C 22+D 22+E 22$ & $={ }^{\prime}$ Nonresp + resp'!N22 \\
\hline 23 & Pu & 238 & $=$ Table51123 & $=$ Table $52^{\prime} 1123$ & $=$ Table711123 & $=C 23+D 23+E 23$ & $={ }^{\prime}$ Nonresp + resp'!N23 \\
\hline 24 & $\mathrm{Pu}$ & 239 & $=$ Table5 1124 & $={ }^{\prime}$ Table $52 ' 1124$ & $=$ Table711124 & $=C 24+D 24+E 24$ & $={ }^{\prime}$ Nonresp + resp'!N24 \\
\hline 25 & Pu mix & $239 / 241$ & & & & & \\
\hline 26 & $\mathrm{Pu}$ & 240 & $=$ Table5.1126 & $={ }^{\prime}$ Table $52^{\prime} \mid 126$ & $=$ Table71!126 & $=C 26+D 26+E 26$ & $=$ 'Nonresp + resp'IN26 \\
\hline 27 & Pu & 241 & $=$ Table5!127 & $={ }^{\circ}$ Table $52^{\prime} \| 27$ & $=$ Table711127 & $=C 27+D 27+E 27$ & $={ }^{\prime}$ Nonresp + resp'!N27 \\
\hline 28 & Pu pure & 242 & $=$ Tables 1128 & $=$ Table $52^{\prime} ! 128$ & $=$ Table7111128 & $=C 28+D 28+E 28$ & $={ }^{\prime}$ Nonresp + resp'IN28 \\
\hline 29 & sr & 90 & $=$ Table5 1129 & $=$ 'Table 52 'll29 & $=$ Table711129 & $=C 29+D 29+E 29$ & 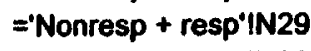 \\
\hline 30 & TC & 99 & $=$ Table5! 130 & $={ }^{\prime}$ Table $52 ' 1130$ & $=$ Table711130 & $=C 30+030+E 30$ & $='$ 'Nonresp + resp'IN30 \\
\hline 31 & Th & 232 & $=$ Table5!131 & $={ }^{\prime}$ Table $52^{\prime} \| 131$ & =Table711131 & $=C 31+D 31+E 31$ & 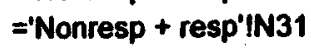 \\
\hline 32 & $\mathrm{TI}$ & 204 & $=$ Table51132 & $={ }^{\prime}$ Table 52 ' 1132 & $=$ Table711132 & $=C 32+D 32+E 32$ & $=$ 'Nonresp + resp'!N32 \\
\hline 33 & $u$ & 235 & $=$ Table5.1133 & $=$ 'Table 52' 1133 & $=$ Table7 11133 & $=C 33+D 33+E 33$ & $={ }^{\prime}$ Nonresp + resp'IN33 \\
\hline 34 & u & 238 & $=$ Table5!134 & $={ }^{\prime}$ Table $52 ' 1134$ & $=$ Table711134 & $=C 34+D 34+E 34$ & 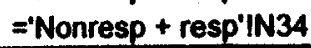 \\
\hline
\end{tabular}




\begin{tabular}{|c|c|c|c|c|c|c|}
\hline & $\bar{A}$ & $\bar{B}$ & $\bar{F}$ & $\overline{\mathbf{G}}$ & $\bar{H}$ & $\bar{L}$ \\
\hline 3 & & & & deconvolut & te mixes & \\
\hline 4 & & & & & & pre-DR \\
\hline 5 & & & mixed & unmix & unmix & unmixed \\
\hline 6 & & & total & Pu239/241 & & total \\
\hline 7 & & & release & & Pu242 & release \\
\hline$\frac{8}{9}$ & Element & At. No. & $\mathrm{Ci}$ & $\mathrm{Ci}$ & $\mathrm{Ci}$ & $\mathrm{Ci}$ \\
\hline 10 & Am & 241 & $1.21 E-02$ & $3.08 E-02$ & $2.52 E-04$ & 4.31E-02 \\
\hline 11 & Am & 243 & $9.68 E-04$ & & & 9.68E-04 \\
\hline 12 & $\mathrm{Ba}$ & 133 & 5.38E-13 & & & $5.38 E-13$ \\
\hline 13 & $\mathrm{Ce}$ & 144 & 1.90E-05 & & & $1.90 E-05$ \\
\hline 14 & cf & 249 & $1.27 E-05$ & & & 1.27E-05 \\
\hline 15 & Cr & 252 & $1.59 E-02$ & & & 1.59E-02 \\
\hline 16 & $\mathrm{~cm}$ & 244 & 2.03E-02 & & & 2.03E-02 \\
\hline 17 & $\mathrm{~cm}$ & 246 & $1.00 E-03$ & & & 1.00E-03 \\
\hline 18 & Co & 60 & 7.19E-01 & & & 7.19E-01 \\
\hline 19 & Cs & 137 & $1.19 E+\infty 0$ & & & 1.19E+00 \\
\hline 20 & $\mathrm{H}$ & 3 & $2.14 E+04$ & & & $2.14 E+04$ \\
\hline 21 & Np & 237 & $4.20 E-05$ & & & $4.20 E-05$ \\
\hline 22 & $P m$ & 147 & 2.52E-08 & & & $2.52 E-08$ \\
\hline 23 & $\mathrm{Pu}$ & 238 & 5.10E-02 & 3.33E-03 & $1.00 E-03$ & 5.53E-02 \\
\hline 24 & $\mathrm{Pu}$ & 239 & $1.78 E-05$ & 9.25E-0.2 & $3.06 \mathrm{E}-06$ & 9.25E-02 \\
\hline 25 & Pu mix & $239 / 241$ & 4.56E-01 & & . & \\
\hline 26 & $\mathrm{Pu}$ & 240 & $9.72 E-07$ & 2.11E-02 & $1.51 E-04$ & 2.12E-02 \\
\hline 27 & $\mathrm{Pu}$ & 241 & 2.91E-01 & $3.08 E-01$ & $1.14 E-02$ & $6.11 E-01$ \\
\hline 28 & Pu mix & 242 & $1.28 E-02$ & & & $1.46 E-04$ \\
\hline 29 & $\mathrm{sr}$ & 90 & $1.18 E+\infty 0$ & & & $1.18 E+00$ \\
\hline 30 & $\mathrm{Tc}$ & 99 & $3.60 \mathrm{E}-13$ & & & $3.60 E-13$ \\
\hline 31 & Th & 232 & 1.93E-06 & & & $1.93 E-06$ \\
\hline 32 & $\mathrm{TI}$ & 204 & $9.00 E-10$ & & & $9.00 E-10$ \\
\hline 33 & $u$ & 235 & $1.78 E-06$ & & & 1.78E-06 \\
\hline 34 & $u$ & 238 & 2.15E-04 & & & 2.15E-04 \\
\hline 35 & $\mathrm{Pu}$ & 242 & & $1.37 E-04$ & $9.45 E-06$ & \\
\hline
\end{tabular}




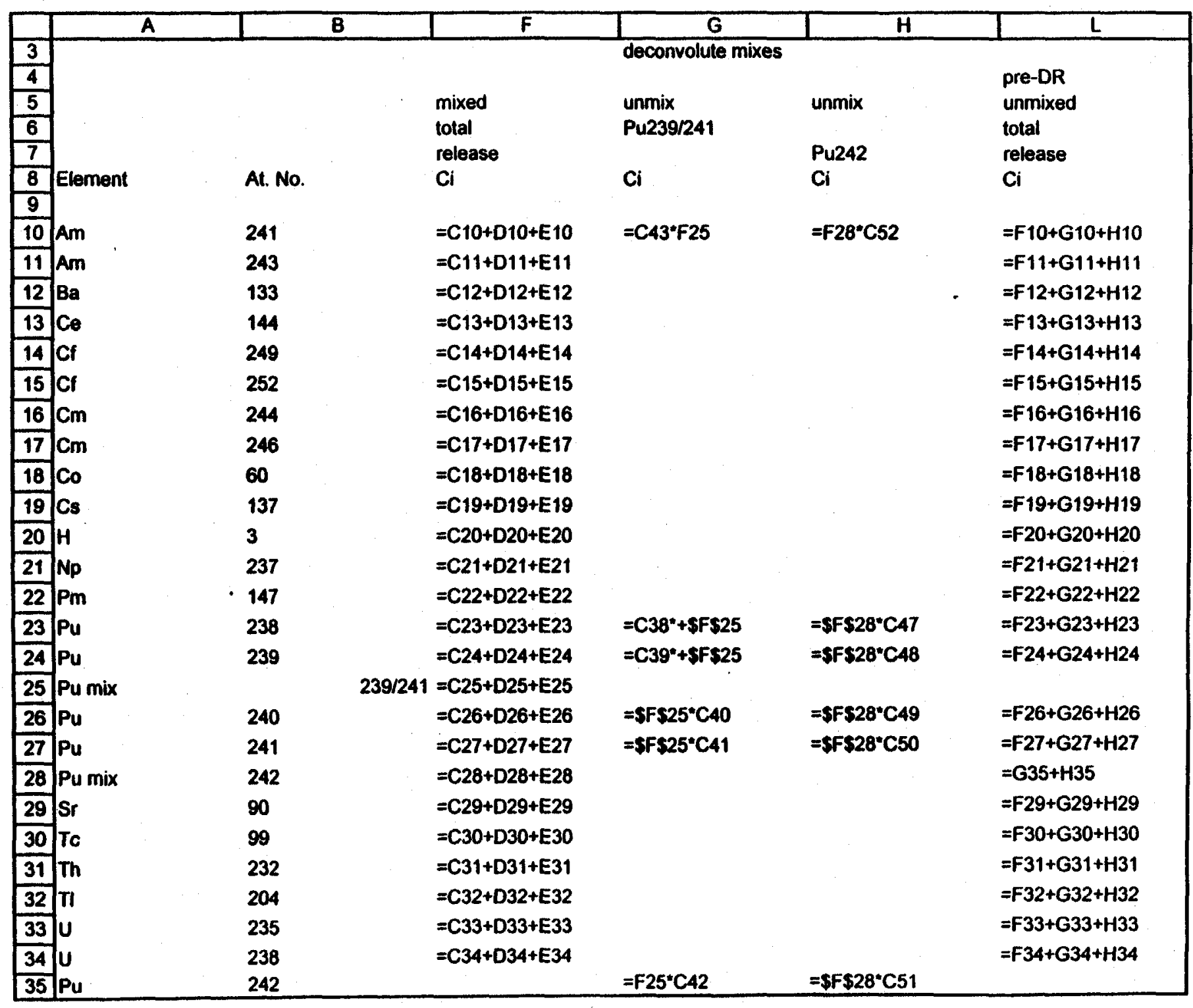




\begin{tabular}{|c|c|c|c|}
\hline & $\bar{A}$ & $\mathbf{B}$ & C \\
\hline 36 & \multicolumn{3}{|c|}{ Pu $239 / 241$ mix activity fractions } \\
\hline 37 & \multicolumn{2}{|l|}{ nuclide } & activity frac \\
\hline 38 & Pu & 238 & 0.0073 \\
\hline 39 & Pu & 239 & 0.2029 \\
\hline 40 & $\mathrm{Pu}$ & 240 & 0.0462 \\
\hline 41 & Pu & 241 & 0.6761 \\
\hline 42 & $\mathbf{P u}$ & 242 & 0.0003 \\
\hline 43 & Am & 241 & 0.0675 \\
\hline 44 & & & \\
\hline 45 & Pu 242 & livity fractions & \\
\hline 46 & nuclide & & activity frac \\
\hline 47 & $\mathbf{P u}$ & 238 & 0.0786 \\
\hline 48 & $\mathrm{Pu}$ & 239 & 0.00024 \\
\hline 49 & $\mathrm{Pu}$ & 240 & 0.01184 \\
\hline 50 & $\mathrm{Pu}$ & 241 & 0.88886 \\
\hline 51 & $\mathrm{Pu}$ & 242 & 0.00074 \\
\hline 52 & Am & 241 & 0.01977 \\
\hline
\end{tabular}




\begin{tabular}{|c|c|c|c|c|c|c|c|}
\hline & A & B & $L$ & $M$ & $N$ & 0 & $\mathbf{P}$ \\
\hline 3 & & & & & & & \\
\hline 4 & & & pre-DR & & DR'd & & \\
\hline 5 & & & unmixed & & unmixed & & \\
\hline 6 & & & & & total & HTO & particulate \\
\hline 7 & & & release & & release & release & release \\
\hline 8 & Element & At. No. & $\mathrm{Ci}$ & $D R=0.75$ & $\mathrm{Ci}$ & $\mathrm{Ci}$ & $\mathrm{Ci}$ \\
\hline 9 & & & & & & & \\
\hline 10 & Am & 241 & 4.31E-02 & 0.75 & 3.23E-02 & & 3.23E-02 \\
\hline 11 & Am & 243 & $9.68 \mathrm{E}-04$ & 0.75 & $7.26 E-04$ & & $7.26 \mathrm{E}-04$ \\
\hline 12 & $\mathrm{Ba}$ & 133 & $5.38 E-13$ & 0.75 & 4.04E-13 & & 4.04E-13 \\
\hline 13 & $\mathrm{Ce}$ & 144 & $1.90 E-05$ & 0.75 & 1.42E-05 & & 1.42E-05 \\
\hline 14 & $\mathrm{Cf}$ & 249 & 1.27E-05 & 0.75 & 9.53E-06 & & 9.53E-06 \\
\hline 15 & Cf & 252 & $1.59 E-02$ & 0.75 & 1.19E-02 & & 1.19E-02 \\
\hline 16 & $\mathrm{Cm}$ & 244 & 2.03E-02 & 0.75 & 1.53E-02 & & 1.53E-02 \\
\hline 17 & $\mathrm{Cm}$ & 246 & $1.00 E-03$ & 0.75 & 7.50E-04 & & $7.50 E-04$ \\
\hline 18 & Co & 60 & 7.19E-01 & 0.75 & 5.40E-01 & & $5.40 E-01$ \\
\hline 19 & Cs & 137 & 1.19E+00 & 0.75 & 8.91E-01 & & 8.91E-01 \\
\hline 20 & $H$ & 3 & $2.14 E+04$ & 0.75 & $1.61 \mathrm{E}+04$ & $1.61 E+04$ & \\
\hline 21 & Np & 237 & 4.20E-05 & 0.75 & 3.15E-05 & & 3.15E-05 \\
\hline 22 & Pm & 147 & 2.52E-08 & 0.75 & 1.69E-08 & & $1.89 E-08$ \\
\hline 23 & Pu & 238 & 5.53E-02 & 0.75 & 4.15E-02 & & 4.15E-02 \\
\hline 24 & $P u$ & 239 & 9.25E-02 & 0.75 & 6.94E-02 & & $6.94 E-02$ \\
\hline 25 & Pu mix & $239 / 241$ & & & & & \\
\hline 26 & $\mathbf{P u}$ & 240 & 2.12E-02 & 0.75 & $1.59 E-02$ & & $1.59 E-02$ \\
\hline 27 & $\mathrm{Pu}$ & 241 & $6.11 E-01$ & 0.75 & 4.58E-01 & & $4.58 E-01$ \\
\hline 28 & Pu mix & 242 & $1.46 \mathrm{E}-04$ & 0.75 & 1.10E-04 & & 1.10E-04 \\
\hline 29 & $\mathrm{Sr}$ & 90 & $1.18 E+00$ & 0.75 & 8.84E-01 & & $8.84 E-01$ \\
\hline 30 & To & 99 & 3.60E-13 & 0.75 & $2.70 \mathrm{E}-13$ & & $2.70 E-13$ \\
\hline 31 & Th & 232 & 1.93E-06 & 0.75 & 1.45E-06 & & $1.45 E-06$ \\
\hline 32 & TI & 204 & $9.00 E-10$ & 0.75 & $6.75 E-10$ & & $6.75 E-10$ \\
\hline 33 & $u$ & 235 & $1.78 E-06$ & 0.75 & 1.34E-06 & & 1.34E-06 \\
\hline 34 & $u$ & 238 & 2.15E-04 & 0.75 & 1.62E-04 & & 1.62E-04 \\
\hline 35 & $\mathrm{Pu}$ & 242 & & & & Sum Ci & $2.96 E+00$ \\
\hline
\end{tabular}




\begin{tabular}{|c|c|c|c|c|c|c|c|}
\hline & $\bar{A}$ & $\bar{B}$ & $L$ & $M$ & $N$ & 0 & $\mathbf{P}$ \\
\hline 3 & & & & & & & \\
\hline 4 & & & pre-DR & & DR'd & & \\
\hline 5 & & & unmixed & & unmixed & & \\
\hline 6 & & & total & & totai & HTO & particulate \\
\hline 7 & & & release & & release & release & release \\
\hline 8 & Element & At. No. & $\mathrm{Ci}$ & $D R=0.75$ & $\mathrm{Ci}$ & $\mathrm{Ci}$ & $\mathrm{Ci}$ \\
\hline 9 & & & & & & & \\
\hline 10 & Am & 241 & $=F 10+G 10+H 10$ & 0.75 & $=L 10^{*} M 10$ & & $=\mathbf{N} 10$ \\
\hline 11 & Am & 243 & $=F 11+G 11+H 11$ & 0.75 & $=L 11 * M 11$ & & $=\mathrm{N} 11$ \\
\hline 12 & $\mathrm{Ba}$ & 133 & $=F 12+G 12+H 12$ & 0.75 & $=L 12^{*} M 12$ & . & $=\mathrm{N} 12$ \\
\hline 13 & $\mathrm{Ce}$ & 144 & $=F 13+G 13+H 13$ & 0.75 & $=L 13^{*} M 13$ & & $=N 13$ \\
\hline 14 & Cf & 249 & $=F 14+G 14+H 14$ & 0.75 & $=L 14^{*} M 14$ & & $=\mathrm{N} 14$ \\
\hline 15 & Cr & 252 & $=F 15+G 15+H 15$ & 0.75 & $=L 15^{*} M 15$ & & $=\mathbf{N} 15$ \\
\hline 16 & $\mathrm{Cm}$ & 244 & $=F 16+G 16+H 16$ & 0.75 & $=L 16 * M 16$ & & $=\mathrm{N} 16$ \\
\hline 17 & $\mathrm{Cm}$ & 246 & $=F 17+G 17+H 17$ & 0.75 & $=L 17^{* M 17}$ & & $=\mathrm{N} 17$ \\
\hline 18 & Co & 60 & $=F 18+G 18+H 18$ & 0.75 & $=L 18^{*} M 18$ & & $=N 18$ \\
\hline 19 & Cs & 137 & $=F 19+G 19+H 19$ & 0.75 & $=L 19^{\star} M 19$ & & $=N 19$ \\
\hline 20 & H & 3 & $=F 20+G 20+H 20$ & 0.75 & $=\mathrm{L} 20^{+} \mathrm{M} 20$ & $=\mathrm{N} 20$ & \\
\hline 21 & No & 237 & $=F 21+G 21+H 21$ & 0.75 & $=L 21 * M 21$ & & $=N 21$ \\
\hline 22 & $\mathrm{Pm}$ & 147 & $=F 22+G 22+H 22$ & 0.75 & $=L .22 * M 22$ & & $=N 22$ \\
\hline 23 & $\mathrm{Pu}$ & 238 & $=F 23+G 23+H 23$ & 0.75 & $=L 23^{*} M 23$ & & $=\mathrm{N} 23$ \\
\hline 24 & $\mathrm{Pu}$ & 239 & $=F 24+G 24+H 24$ & 0.75 & $=L 24^{*} M 24$ & & $=N 24$ \\
\hline 25 & Pu mix & & & & & . & \\
\hline 26 & $\mathrm{Pu}$ & 240 & $=F 26+G 26+H 26$ & 0.75 & $=L .26^{*} M 26$ & & $=\mathrm{N} 26$ \\
\hline 27 & $\mathrm{Pu}$ & 241 & $=F 27+G 27+H 27$ & 0.75 & $=L 27^{*} \mathrm{M} 27$ & & $=N 27$ \\
\hline 28 & Pu mix & 242 & $=\mathrm{G} 35+\mathrm{H} 35$ & 0.75 & $=L 28^{*} M 28$ & & $=\mathrm{N} 28$ \\
\hline 29 & $\mathrm{sr}$ & 90 & $=F 29+G 29+H 29$ & 0.75 & $=L .29+M 29$ & & $=\mathrm{N} 29$ \\
\hline 30 & TC & 99 & $=F 30+G 30+H 30$ & 0.75 & $=\mathrm{L} \cdot 30^{*} \mathrm{M} 30$ & & $=\mathrm{N} 30$ \\
\hline 31 & Th & 232 & $=F 31+G 31+H 31$ & 0.75 & $=L 31^{*} M 31$ & & $=N 31$ \\
\hline 32 & $\mathrm{TI}$ & 204 & $=F 32+G 32+H 32$ & 0.75 & $=L 32^{* M} M 32$ & & $=N 32$ \\
\hline 33 & $\mathbf{u}$ & 235 & $=F 33+G 33+H 33$ & 0.75 & $=L 33^{*} M 33$ & & $=\mathrm{N} 33$ \\
\hline 34 & $\mathbf{u}$ & 238 & $=F 34+G 34+H 34$ & 0.75 & $=L 34^{\prime \prime M} 34$ & & $=N 34$ \\
\hline 35 & $\mathrm{Pu}$ & 242 & & & & Sum Ci & $=S U M(P 10: P 34)$ \\
\hline
\end{tabular}




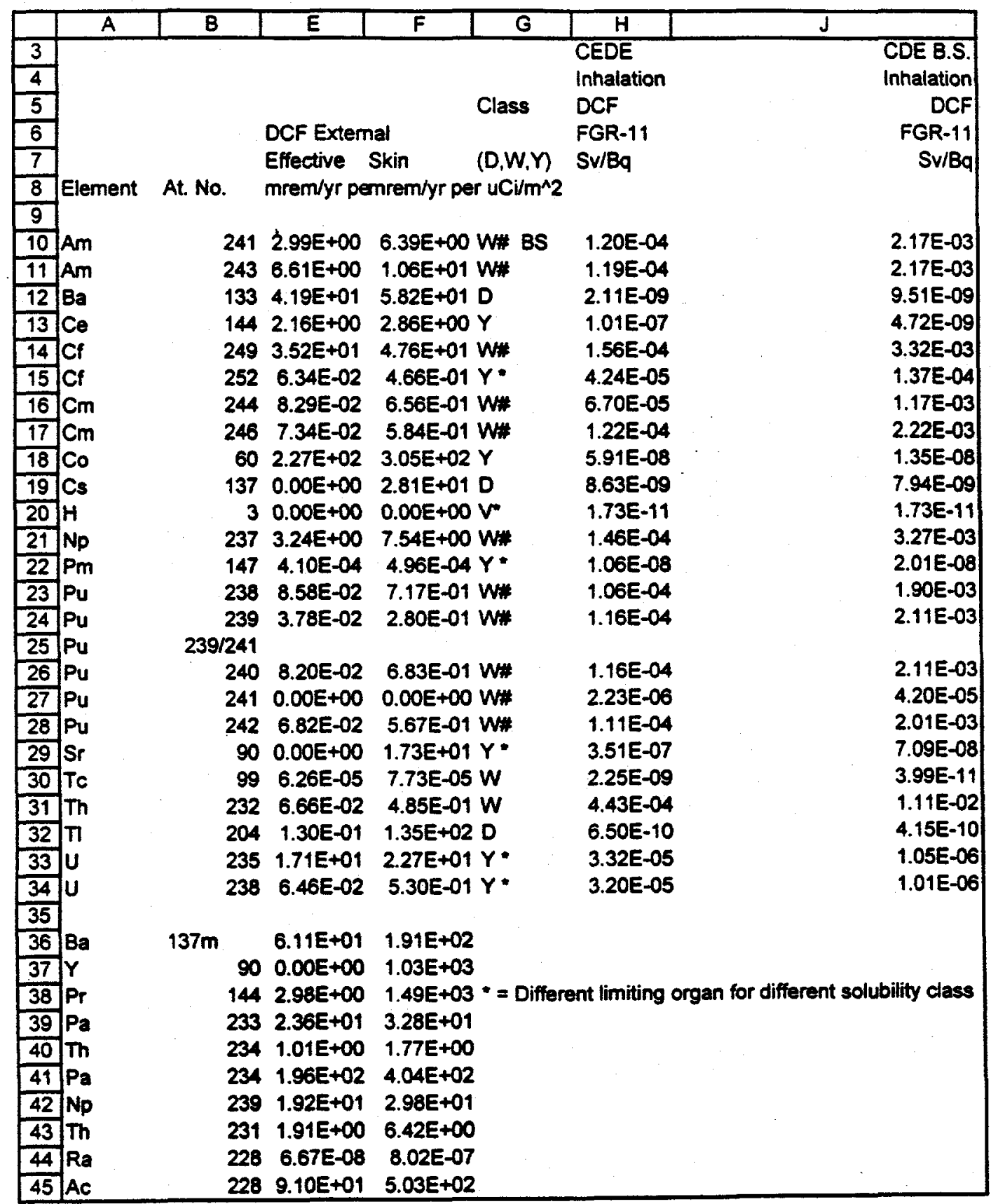




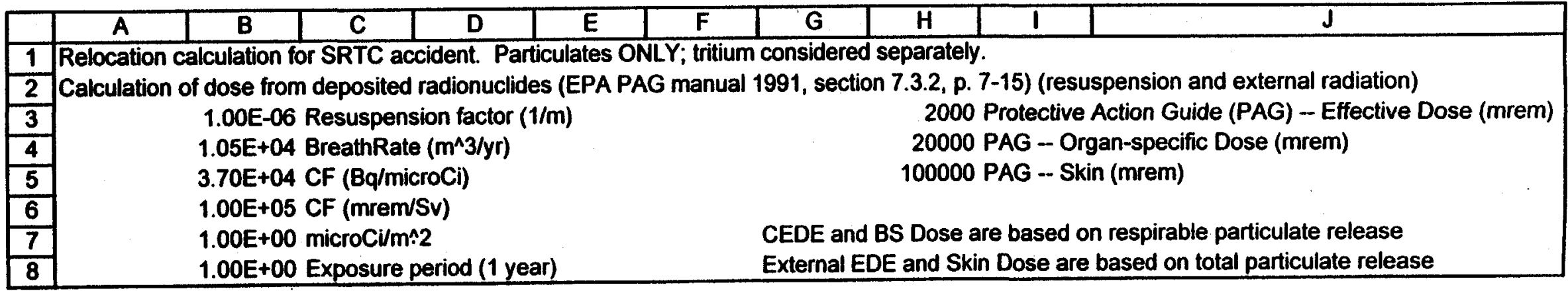




\begin{tabular}{|c|c|c|c|}
\hline & $\bar{A}$ & $\bar{B}$ & C \\
\hline$\theta$ & & $\begin{array}{l}\text { Total } \\
\text { Release } \\
\text { (Ci) }\end{array}$ & $\begin{array}{l}\text { Activity } \\
\text { fraction } \\
\text { (Total) }\end{array}$ \\
\hline 10 & Am-241 & 3.23E-02 & $1.09 E-02$ \\
\hline 11 & Am-243 & $7.26 E-04$ & $2.45 E-0.4$ \\
\hline 12 & Ba-133 & 4.04E-13 & $1.36 E-13$ \\
\hline 13 & Ce-144 & $1.42 E-05$ & $4.80 E-06$ \\
\hline 14 & Cf-249 & 9.53E-06 & $3.22 E-06$ \\
\hline 15 & $C 1-252$ & $1.19 E-02$ & $4.03 E-03$ \\
\hline 16 & Cm-244 & $1.53 E-02$ & $5.15 E-03$ \\
\hline 17 & $\mathrm{Cm}-246$ & $7.50 E-04$ & 2.53E-04 \\
\hline 18 & Co-60 & $5.40 E-01$ & $1.82 E-01$ \\
\hline 19 & Cs-137 & 8.91E-01 & 3.01E-01 \\
\hline 20 & $\mathrm{H}-3$ & $0.00 E+\infty 0$ & $0.00 E+\infty$ \\
\hline 21 & $\mathrm{~Np}-237$ & 3.15E-05 & $1.06 \mathrm{E}-05$ \\
\hline 22 & $P m-147$ & $1.89 E-08$ & $6.38 E-09$ \\
\hline 23 & Pu-238 & $4.15 E-02$ & $1.40 E-02$ \\
\hline 24 & Pu-239 & $6.94 E-02$ & 2.34E-02 \\
\hline 25 & Pu mix-239/241 & $0.00 E+\infty 0$ & $0.00 E+\infty$ \\
\hline 26 & Pu-240 & $1.59 E-02$ & $5.37 E-03$ \\
\hline 27 & Pu-241 & 4.58E-01 & 1.55E-01 \\
\hline 28 & Pu pure-242 & $1.10 E-04$ & $3.70 E-05$ \\
\hline 29 & Sr-90 & 8.84E-01 & $2.99 E-01$ \\
\hline 30 & Tc-99 & 2.70E-13 & $9.12 E-14$ \\
\hline 31 & Th-232 & $1.45 E-06$ & 4.90E-07 \\
\hline 32 & $T 1-204$ & $6.75 E-10$ & 2.28E-10 \\
\hline 33 & U-235 & $1.34 E-06$ & $4.52 E-07$ \\
\hline 34 & U-238 & $1.62 \mathrm{E}-04$ & $5.45 E-05$ \\
\hline 35 & Total & $2.96 E+00$ & $1.00 E+\infty$ \\
\hline 36 & Ba-137m & 8.91E-01 & $3.01 E-01$ \\
\hline 37 & $Y-90$ & $8.84 E-01$ & 2.99E-01 \\
\hline 38 & Pr-144 & 1.42E-05 & $4.80 E-06$ \\
\hline 39 & Pa-233 & 3.15E-05 & $1.06 E-05$ \\
\hline 40 & Th-234 & $1.62 E-04$ & 5.45E-05 \\
\hline 41 & Pa-234 & $1.62 E-04$ & 5.45E-05 \\
\hline 42 & Np-239 & 7.26E-04 & $2.45 E-04$ \\
\hline 43 & Th-231 & 1.34E-08 & $4.52 E-07$ \\
\hline 44 & $R a-228$ & 1.45E-06 & 4.90E-07 \\
\hline$\frac{45}{46}$ & $A c-228$ & $1.45 E-06$ & $4.90 \mathrm{E}-07$ \\
\hline 47 & Total & & \\
\hline
\end{tabular}




\begin{tabular}{|c|c|c|}
\hline & $A$ & C \\
\hline 9 & Total Release (Ci) & $\begin{array}{l}\text { Activity fraction } \\
\text { (Total) }\end{array}$ \\
\hline 10 & 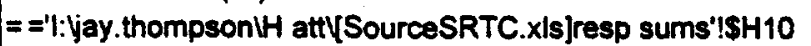 & $=\mathrm{B} 10 / \$ B \$ 35$ \\
\hline 11 & $==' \mid$ :yay.thompsonUH attuSourceSRTC.xIs]resp sums'!SH11 & $=\mathrm{B} 11 / \mathrm{SB} \$ 35$ \\
\hline 12 & 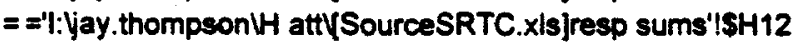 & $=812 / \$ B \$ 35$ \\
\hline 13 & $==' \mid$ :yay thompson $H$ att $[$ SourceSRTC.xIs]resp sums'!SH13 & $=\mathrm{B} 13 / \mathrm{SB} \$ 35$ \\
\hline 14 & $==1$ : :jay , thompsonWH attySourceSRTC.xls]resp sums'!SH14 & $=814 / \$ 8 \$ 35$ \\
\hline 15 & $==' 1$ : ljay.thompsonUH attiSourceSRTC.xls\}resp sums'!SH15 & $=B 15 / \$ B \$ 35$ \\
\hline 16 & $==$ ='1:'yay.thompsoniH attVSourceSRTC.xIs]resp sums'!SH16 & $=\mathrm{B} 16 / \mathrm{SB} \$ 35$ \\
\hline 17 & $===^{\prime} \mid$ : yay.thompson'H attVSourceSRTC.xIs]resp sums'!\$H17 & $=B 17 / \$ B \$ 35$ \\
\hline 18 & 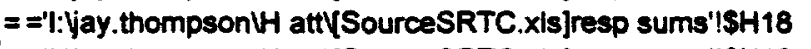 & $=B 18 / \$ 8 \$ 35$ \\
\hline 19 & $===^{\prime} \mid$ :yay thompson $\mathrm{H}$ attVSourceSRTC.xIs]resp sums'!SH19 & $=B 19 / 5 B S 35$ \\
\hline 20 & & $=\mathrm{B} 20 / \$ \mathrm{~B} \$ 35$ \\
\hline 21 & $===^{\prime}$ : :jay.thompsonUH attySourceSRTC.xls]resp sums'ISH21 & $=\mathrm{B} 21 / \$ 8 \$ 35$ \\
\hline 22 & $==' 1:$ yay.thompson $H$ attYSourceSRTC.X|s]resp sums'!SH22 & $=822 / \$ B \$ 35$ \\
\hline 23 & $===^{\prime} \mid$ : yay thompson'H attySourceSRTC.xis\}resp sums'!\$H23 & $=823 / 5 B \$ 35$ \\
\hline 24 & 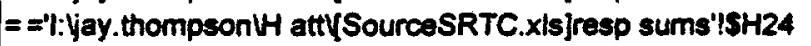 & $\Rightarrow B 24 / \$ B S 35$ \\
\hline 25 & $===^{\prime} \mid$ :yay.thompson $1 H$ attVSourceSRTC.xls\}resp sums'!SH25 & $=B 25 / \$ B \$ 35$ \\
\hline 26 & = ='l:|yay.thompsonUH attySourceSRTC.xIs]resp sums'!\$H26 & $=826 / \$ B \$ 35$ \\
\hline 27 & 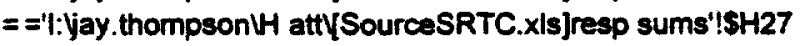 & $=827 / \$ B \$ 35$ \\
\hline 28 & 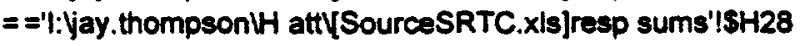 & $=\mathrm{B} 28 / \$ \mathrm{~B} \$ 35$ \\
\hline 29 & $===^{\prime} 1$ : Yay.thompsonUH attVSourceSRTC.xIs]resp sums'!SH29 & $=B 29 / \$ B \$ 35$ \\
\hline 30 & $==' 1$ : yay. thompsonUH attVSourceSRTC.XIs]resp sums'!\$H30 & $=\mathrm{B} 30 / \$ \mathrm{~B} \$ 35$ \\
\hline 31 & $=$ ='I: yay.thompsonlH attVSourceSRTC.xIs]resp sums'!SH31 & $=\mathrm{B} 31 / \$ \mathrm{~B} \$ 35$ \\
\hline 32 & $==' 1:$ iay.thompson $\mathrm{H}$ attVSourcesRTC.xIs]resp sums'!SH32 & $=B 32 / \$ B \$ 35$ \\
\hline 33 & 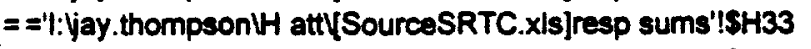 & $=B 33 / \$ B \$ 35$ \\
\hline 34 & $=='$ : Yjay.thompsoniH attYSourceSRTC.xls]resp sums'!\$H34 & $=B 34 / \$ B \$ 35$ \\
\hline 35 & $=$ SUM(B10:B34) & $=$ SUM(C10:C34) \\
\hline 36 & $==819$ & $=\mathrm{C} 19$ \\
\hline 37 & $==\mathbf{B 2 9}$ & $=C 29$ \\
\hline 38 & $==813$ & $=\mathrm{C} 13$ \\
\hline 39 & $==821$ & $=\mathrm{C} 21$ \\
\hline 40 & $==834$ & $=\mathrm{C} 34$ \\
\hline 41 & $==B 34$ & $=c 34$ \\
\hline 42 & $==B 11$ & $=C 11$ \\
\hline 43 & $==833$ & $=C 33$ \\
\hline 44 & $==831$ & $=$ C.31 \\
\hline$\frac{45}{46}$ & $==831$ & $=C 31$ \\
\hline 47 & & \\
\hline
\end{tabular}




\begin{tabular}{|c|c|c|c|}
\hline & $\bar{A}$ & $\bar{D}$ & $\bar{E}$ \\
\hline 9 & & $\begin{array}{l}\text { Respirable } \\
\text { Release (Ci) }\end{array}$ & $\begin{array}{l}\text { Activity } \\
\text { fraction } \\
\text { (Resp) }\end{array}$ \\
\hline 10 & Am-241 & $1.49 E-02$ & $5.56 \mathrm{E}-03$ \\
\hline 11 & Am-243 & 4.55E-04 & $1.70 E-04$ \\
\hline 12 & Ba-133 & $4.04 E-13$ & 1.51E-13 \\
\hline 13 & Ce-144 & $1.42 E-05$ & $5.32 E-06$ \\
\hline 14 & CF-249 & $8.24 E-06$ & $3.08 E-06$ \\
\hline 15 & $C f-252$ & $1.19 E-02$ & 4.47E-03 \\
\hline 16 & $\mathrm{Cm}-244$ & $1.71 E-02$ & $6.40 E-03$ \\
\hline 17 & $\mathrm{Cm}-246$ & 4.99E-04 & $1.87 E-04$ \\
\hline 18 & Co-60 & 5.40E-01 & 2.02E-01 \\
\hline 19 & Cs-137 & 8.91E-01 & $3.34 E-01$ \\
\hline 20 & $\mathrm{H}-3$ & $0.00 E+\infty 0$ & $0.00 E+\infty$ \\
\hline 21 & Np-237 & 2.44E-05 & $9.14 E-06$ \\
\hline 22 & $\mathrm{Pm}-147$ & $1.89 E-08$ & 7.07E-09 \\
\hline 23 & Pu-238 & 1.81E-02 & $6.77 E-03$ \\
\hline 24 & Pu-239 & $2.35 E-02$ & $8.78 E-03$ \\
\hline 25 & Pu mix-239/241 & $0.00 E+\infty$ & $0.00 E+\infty$ \\
\hline 26 & Pu-240 & 5.34E-03 & $2.00 E-03$ \\
\hline 27 & Pu-241 & 2.66E-01 & $9.97 E-02$ \\
\hline 28 & Pu pure-242 & 3.47E-05 & 1.30E-05 \\
\hline 29 & Sr-90 & 8.83E-01 & $3.30 E-01$ \\
\hline 30 & TC-99 & $2.70 E-13$ & $1.01 E-13$ \\
\hline 31 & Th-232 & $1.44 E-06$ & 5.38E-07 \\
\hline 32 & Ti-204 & $6.75 E-10$ & $2.53 E-10$ \\
\hline 33 & U-235 & $6.88 E-07$ & $2.57 E-07$ \\
\hline 34 & $U-238$ & 5.11E-05 & $1.91 E-05$ \\
\hline 35 & Total & $2.67 E+00$ & $1.00 E+\infty$ \\
\hline 36 & Ba-137m & 8.91E-01 & 3.34E-01 \\
\hline 37 & $Y-90$ & 8.83E-01 & 3.30E-01 \\
\hline 38 & Pr-144 & $1.42 E-05$ & $5.32 E-06$ \\
\hline 39 & Pa-233 & 2.44E-05 & $9.14 E-06$ \\
\hline 40 & Th-234 & $5.11 E-05$ & $1.91 E-05$ \\
\hline 41 & Pa-234 & 5.11E-05 & 1.91E-05 \\
\hline 42 & Np-239 & 4.55E-04 & $1.70 E-04$ \\
\hline 43 & Th-231 & $6.88 E-07$ & 2.57E-07 \\
\hline 44 & Ra-228 & $1.44 E-06$ & 5.38E- -07 \\
\hline$\frac{45}{16}$ & AC-228 & 1.44E-06 & $5.38 E-07$ \\
\hline 47 & Total & & \\
\hline
\end{tabular}




\begin{tabular}{|c|c|c|}
\hline & $\mathrm{D}$ & $\bar{E}$ \\
\hline 9 & Respirable Release (Ci) & $\begin{array}{l}\text { Activity fraction } \\
\text { (Resp) }\end{array}$ \\
\hline 10 & 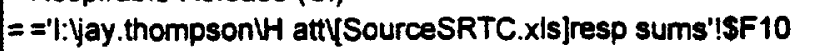 & $=010 / \$ 0 \$ 35$ \\
\hline 11 & $==' \mid$ :yay.thompsonUH attySourceSRTC.xis]resp sums'ISF11 & $=011 / \$ 0 \$ 35$ \\
\hline 12 & $==' I:$ yay. thompsonUH attYSourceSRTC.xis|resp sums'!\$F12 & $=012 / \$ D \$ 35$ \\
\hline 13 & $==" 1$ :yay.thompsonUH attVSourceSRTC.xis]resp sums'!\$F 13 & $=013 / \$ D \$ 35$ \\
\hline 14 & $=='$ : :jay.thompsoniH attVSourceSRTC.xis]resp sums'!SF14 & $=D 14 / \$ D S 35$ \\
\hline 15 & $==^{\prime}$ ': :jay.thompsoniH attVSourceSRTC.xIsjresp sums'!SF15 & $=015 / \$ D \$ 35$ \\
\hline 16 & $==' !:$ yay. thompsonUH attySourceSRTC.xisiresp sums'!\$F 16 & $=016 / \$ 0 \$ 35$ \\
\hline 17 & $=={ }^{\prime}$ I: Yay.thompsoniH attYSourceSRTC.xIs]resp sums'!SF17 & $=D 17 / S D S 35$ \\
\hline 18 & $=='$ : :jay.thompsonUH attuSourceSRTC.xisjresp sums'!SF18 & $=D 18 / 50 \$ 35$ \\
\hline 19 & $=$ ='I:yay.thompsonUH attVSourceSRTC.xIs]resp sums'!SF19 & $=D 19 / \$ D \$ 35$ \\
\hline 20 & $=0$ & $=020 / \$ 0 \$ 35$ \\
\hline 21 & 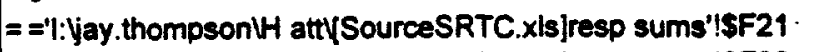 & $=021 / \$ D \$ 35$ \\
\hline 22 & $=$ ='I: ljay. thompsonUH attySOurceSRTC.xIsjresp sums'!SF22 & $=022 / \$ D \$ 35$ \\
\hline 23 & $==$ 'I: :jay. thompsonWH attySourceSRTC.xis]resp sums'!SF23 & $=023 / 50 \$ 35$ \\
\hline 24 & $==1:$ :jay.thompsonUH attVSourceSRTC.xis]resp sums'!\$F24 & $=024 / \$ D S 35$ \\
\hline 25 & $==1:$ :yay.thompsonUH attVSSourceSRTC.x/s]resp sums'!SF25 & $=D 25 / \$ D \$ 35$ \\
\hline 26 & $==$ 'I: jay.thompsonWH attVSourceSRTC.XIs]resp sums'ISF26 & $=\mathrm{D} 26 / 5 \mathrm{D} \$ 35$ \\
\hline 27 & $===^{\prime}:$ : jay.thompsonUH attVSourceSRTC.xis]resp sums' $\$ \$ F 27$ & $=\mathrm{D} 27 / \$ \mathrm{D} \$ 35$ \\
\hline 28 & $=={ }^{\prime} 1$ : Yjay.thompson $H$ attVSOUrceSRTC.xis]resp sums'!SF28 & $=D 28 / \$ D \$ 35$ \\
\hline 29 & $==' 1$ :yay.thompsonUH attUSourceSRTC.xIs]resp sums'!SF29 & $=D 29 / \$ D \$ 35$ \\
\hline 30 & $==$ '!:yay.thompsoniH attVSourceSRTC.xIs]resp sums'!SF30 & $=030 / \$ 0 \$ 35$ \\
\hline 31 & $==1:$ :yay.thompsontH attVSourceSRTC.Xis]resp sums'!\$F31 & $=D 31 / \$ D \$ 35$ \\
\hline 32 & $==' 1:$ yay thompsoniH attl[SourceSRTC.xls]resp sums'!\$F32 & $=032 / \$ D \$ 35$ \\
\hline 33 & $==' 1:$ Jay. thompsonUH attySourceSRTC.xIsjresp sums'!SF33 & $=D 33 / \$ D \$ 35$ \\
\hline 34 & $==^{\prime}$ : $:$ jay.thompsonU attiSourceSRTC.xIs]resp sums'!SF34 & $=\mathrm{D} 34 / \$ \mathrm{D} \$ 35$ \\
\hline 35 & $=$ SUM(010:D34) & $=\operatorname{SUM}(E 10: E 34)$ \\
\hline 36 & $==019$ & $=E 19$ \\
\hline 37 & $==\mathrm{D} 29$ & $=E 29$ \\
\hline 38 & $==013$ & $=E 13$ \\
\hline 39 & $==021$ & $=\mathbf{E 2 1}$ \\
\hline 40 & $==034$ & $=E 34$ \\
\hline 41 & $==034$ & $=E 34$ \\
\hline 42 & $=0011$ & $=E 11$ \\
\hline 43 & $==033$ & $=E 33$ \\
\hline 44 & $==031$ & $=$ E31 \\
\hline 45 & $==031$ & $=\mathrm{E} 31$ \\
\hline$\frac{46}{47}$ & & \\
\hline
\end{tabular}




\begin{tabular}{|c|c|c|c|}
\hline & $\bar{A}$ & $\mathbf{G}$ & $\bar{H}$ \\
\hline 9 & & $\begin{array}{l}\text { CEDE } \\
\text { (mrem per } \\
\mathrm{uCl} / \mathrm{m}^{\wedge} 2 \text { ) }\end{array}$ & $\begin{array}{l}\text { External } \\
\text { EDE } \\
\text { (mrem per } \\
\text { uCi/m^2) }\end{array}$ \\
\hline 10 & Am-241 & $2.59 E+01$ & $3.62 E-02$ \\
\hline 11 & Am-243 & $7.87 E-01$ & $1.80 \mathrm{E}-03$ \\
\hline 12 & $B a-133$ & 1.24E-14 & 6.33E-12 \\
\hline 13 & $\mathrm{Ce}-144$ & 2.09E-05 & 1.15E-05 \\
\hline 14 & Cf-249 & $1.87 E-02$ & $1.26 E-04$ \\
\hline 15 & $C f-252$ & $7.36 E+\infty 0$ & $2.83 E-04$ \\
\hline 16 & $\mathrm{Cm}-244$ & $1.67 E+01$ & 4.73E-04 \\
\hline 17 & $\mathrm{Cm}-246$ & $8.85 E-01$ & 2.06E-05 \\
\hline 18 & Co-60 & 4.64E-01 & $4.58 E+01$ \\
\hline 19 & Cs-137 & 1.12E-01 & $0.00 E+\infty$ \\
\hline 20 & $\mathrm{H}-3$ & $0.00 E+00$ & $0.00 E+\infty$ \\
\hline 21 & Np-237 & 5.19E-02 & 3.82E-05 \\
\hline 22 & Pm-147 & 2.91E-09 & $2.90 E-12$ \\
\hline 23 & $P u-238$ & $2.79 E+01$ & 1.33E-03 \\
\hline 24 & Pu-239 & $3.96 E+01$ & 9.81E-04 \\
\hline 25 & Pu mix-239/241 & $0.00 E+\infty$ & $0.00 E+\infty$ \\
\hline 26 & Pu-240 & $9.01 E+\infty 0$ & 4.88E-04 \\
\hline 27 & Pu-241 & $8.64 E+\infty 0$ & $0.00 E+00$ \\
\hline 28 & Pu pure-242 & 5.60E-02 & $2.80 \mathrm{E}-06$ \\
\hline 29 & Sr-90 & $4.51 E+\infty 0$ & $0.00 E+\infty$ \\
\hline 30 & Tc-99 & 8.83E-15 & $6.32 E-18$ \\
\hline 31 & Th-232 & $9.26 E-03$ & 3.62E-08 \\
\hline 32 & $\pi 7-204$ & 6.38E-12 & 3.28E-11 \\
\hline 33 & U-235 & 3.32E-04 & 8.57E-06 \\
\hline 34 & $\mathrm{U}-238$ & 2.38E-02 & . $3.90 E-06$ \\
\hline 35 & Total & & \\
\hline 36 & $\mathrm{Ba}-137 \mathrm{~m}$ & & $2.04 E+01$ \\
\hline 37 & $Y-90$ & & $0.00 E+00$ \\
\hline 38 & Pr-144 & & 1.59E-05 \\
\hline 39 & Pa-233 & & $2.78 E-04$ \\
\hline 40 & Th-234 & & 6.10E-05 \\
\hline 41 & $\mathrm{~Pa}-234$ & & $-1.18 E-02$ \\
\hline 42 & Np-239 & & 5.22E-03 \\
\hline 43 & Th-231 & & 9.57E-07 \\
\hline 44 & Ra-228 & & $3.62 E-14$ \\
\hline 45 & $A c-228$ & & 4.94E-05 \\
\hline 46 & & & \\
\hline 47 & Total & $1.42 E+02$ & $6.63 E+01$ \\
\hline
\end{tabular}




\begin{tabular}{|c|c|c|}
\hline & A] & $\mathrm{H}$ \\
\hline $\mathbf{9}$ & CEDE (mrem per uCl//n^2) & External EDE (mrem per $\left.u C i / m^{\wedge} 2\right)$ \\
\hline 10 & $=^{\prime}=\$ B \$ 7^{*} \$ B \$ 3^{*} \$ B \$ 4^{*} \$ B \$ 5^{*} \$ B \$ 6^{*} \$ B \$ 8^{*} E 10^{*}$ Relocation!H10 & $=\$ B \$ 7^{\circ} \$ B \$ 8^{*} \$ C 10^{*}$ Relocation!E $10^{*} \$ B \$ 35 /$ \$D $\$ 35$ \\
\hline 11 & $==^{\prime}=\$ B \$ 7^{*} \$ B \$ 3 * \$ B \$ 4 * \$ B \$ 5 * \$ B \$ 6 * \$ B \$ 8 * E 11^{*}$ Relocation!H11 & $=\$ B \$ 7 * \$ B \$ 8 *$ \$C11*Relocation!E11*\$B\$35/\$D\$35 \\
\hline 12 & $=\$ B \$ 7^{*} \$ B \$ 3^{*} \$ B \$ 4^{*} \$ B S 5 * \$ B \$ 6^{*} \$ B \$ 8^{*} E 12^{*}$ Relocation!H12 & $=\$ B \$ 7^{*} \$ B \$ 8^{*} \$ C 12^{*}$ Relocation!E12*\$B\$35/\$D 33 S \\
\hline 13 & $='=\$ B \$ 7^{*} \$ B \$ 3 * \$ B S 4 * \$ B \$ 5 " \$ B \$ 6 * \$ B \$ 8^{*}$ E13*Relocation!H13 & $=\$ B \$ 7^{*} \$ B \$ 8 * \$ C 13^{*}$ Relocation!E13*\$B \$35/\$D \$35 \\
\hline 14 & 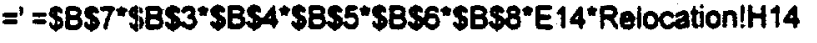 & $=\$ B \$ 7^{*} \$ B \$ 8 * \$ C 14^{*}$ Relocation!E14*\$B\$35/\$D\$35 \\
\hline 15 & $==^{\prime}=\$ B \$ 7^{*} \$ B \$ 3^{*} \$ B \$ 4 * \$ B 5^{*} \$ B \$ 6^{*} \$ B \$ 8^{*}$ E15*Relocation!H15 & $=\$ B \$ 7^{*}$ \$BS8*\$C15*Relocation!E15*\$B\$35/\$D\$35 \\
\hline & $=^{\prime}=\$ B \$ 7^{*} \$ B \$ 3^{*} \$ B \$ A^{*} \$ B \$ 55^{*} \$ B \$ 6^{*} \$ B \$ 8^{*} E 1$ & $=\$ B \$ 7^{*} \$ B \$ 8 * \$ C 16^{*}$ Relocation!E16*\$B\$35/\$D\$35 \\
\hline & $==^{\prime}=\$ B \$ 7^{*} \$ B \$ 3^{*} \$ B \$ 44^{*} \$ B \$ 5 * \$ B S 6^{*} \$ B \$ 8^{*} E 17^{*} R$ & $=\$ B 57^{* \$ B S 88} "$ SC17"Relocation!E17"\$B\$35/\$O\$35 \\
\hline 18 & 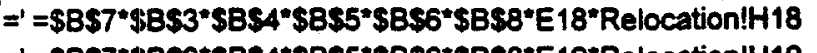 & $=\$ B S 7^{*}$ SBSB* $\$ C 18^{*}$ RelocationIE18*\$B\$35/\$D\$35 \\
\hline 19 & $=l^{\prime}=\$ B \$ 7^{*} \$ B \$ 3^{*} \$ B S 4^{*} \$ B \$ 5^{*} \$ B \$ 6^{*} \$ B S 8^{*} E 19^{*}$ Relocation!H19 & $=\$ B \$ 7^{*}$ SBS8*\$C19*Relocation!E19'\$B\$35/SD\$35 \\
\hline & $==^{\prime}=\$ B \$ 7^{*} \$ B \$ 3^{*} \$ B \$ 4^{*} \$ B \$ 5^{\circ} \$ B \$ 6^{*} \$ B \$ 8^{*} E 2$ & $=\$ B \$ 7^{*} \$ B \$ 8^{*} \$ C 20^{*}$ Relocation!E20*\$B\$35/\$D\$33 \\
\hline & 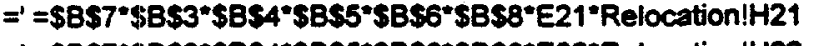 & $=\$ B \$ 7^{*}$ SBS8*\$C21*Relocation!E21*\$BS35/SD\$33 \\
\hline 22 & $==^{\prime}=\$ B \$ 7^{*} \$ B \$ 3^{*} \$ B S 4^{*} \$ B \$ 5^{*} \$ B \$ 6^{*} \$ B \$ 8^{*} E 22^{*}$ Relocation!H22 & $=\$ B \$ 7^{*} \$ B \$ 8^{*} \$ C 22^{*}$ Relocation!E22*\$B\$35/\$D\$3 S \\
\hline 23 & $={ }^{\prime}=\$ B \$ 7^{*} \$ B \$ 3^{*} \$ B \$ 4^{*} \$ B \$ 5^{*} \$ B \$ 6 * \$ B \$ 8^{*} E 23^{*}$ Relocation!H23 & $=$ SBS7* \$BS8* SC23*Relocation!E23*\$BS35/SD\$35 \\
\hline 24 & $==^{\prime}=\$ B S 7^{*} \$ B \$ 3^{*} \$ B \$ 4^{*} \$ B \$ 55^{*} \$ B \$ 6^{*} \$ B \$ 8^{*} E 24$ & $=\$ B \$ 7^{*} \$ B \$ 8^{*} \$ C 24^{*}$ Relocation!E24"\$B\$35/\$D\$3S \\
\hline & 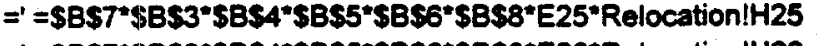 & $=\$ B \$ 7^{*}$ SBS8*\$C25*Relocation!E25*\$BS35/SD\$35 \\
\hline & 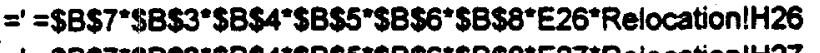 & $=\$ 8 \$ 7^{*} \$ B \$ 8^{*} \$ C 26^{*}$ Relocation!E26*\$B\$35/\$D\$35 \\
\hline & $=^{\prime}=\$ B \$ 7^{*} \$ B \$ 3^{*} \$ B \$ 4^{*} \$ B \$ 5^{*} \$ B \$ 6 * \$ B \$ 8^{*} E 27$ & $=\$ 8 \$ 7^{*} \$ B \$ 8 *$ \$C27*RelocationlE27*\$B\$35/\$D\$35 \\
\hline 28 & $==^{\prime}=\$ B \$ 7^{*} \$ B \$ 3^{*} \$ B \$ 4^{*} \$ B \$ 5^{*} \$ B \$ 6 * \$ B \$ 8^{*} E 28^{*}$ & $=\$ 8 \$ 7^{*}$ \$B\$8*\$C28*Relocation!E28*\$B\$35/\$D \$35 \\
\hline 29 & $=^{\prime}=\$ B \$ 7^{*} \$ B S \$ 3^{*} \$ B \$ 4^{*} \$ B \$ 5^{*} \$ B S 6 * \$ B S 8^{*} E 29^{*}$ Relocation!H29 & $=\$ 8 \$ 7^{*} \$ B \$ 8 * \$ C 29 *$ Relocation!E29*\$8\$35/\$D\$35 \\
\hline 30 & $=^{\prime}=\$ B \$ 7^{*}$ \$BS3*\$BS4"\$B\$5"\$B\$6"\$BS8*E30"Relocation!H30 & $=\$ B \$ 7^{*}$ \$BS8*\$C30*Relocation!E30*\$B\$35/\$D\$3 \\
\hline 31 & $=^{\prime}=\$ B \$ 7^{*} \$ B \$ 3^{*} \$ B \$ 4^{*} \$ B \$ 5^{*} \$ 8 \$ 66^{*} \$ B \$ 8^{*} E 31^{*}$ Reloca & $=\$ B \$ 7^{*}$ \$B\$8*\$C31"Relocation!E31"\$B\$35/\$D \$35 \\
\hline 32 & $==^{\prime}=\$ B \$ 7^{*} \$ B \$ 33^{*} \$ B \$ 4^{*} \$ B \$ 55^{*} \$ B \$ 66^{*} \$ B \$ 8^{*} E 32^{*} R$ & $=\$ B \$ 7^{*}$ \$BS8* $\$$ C32*Relocation!E32*\$B\$35/\$D\$35 \\
\hline 33 & $==^{\prime}=\$ B \$ 7^{*} \$ B \$ 3^{*} \$ B \$ 4^{*} \$ 8 \$ 5^{*} \$ B \$ 66^{*} \$ B \$ 8^{*} E 33^{*}$ Rel & $=\$ B \$ 7^{*} \$ 8$ S8* $\$ C 33^{*}$ Relocation!E33*\$B \$35/\$D\$35 \\
\hline$\frac{34}{35}$ & $==^{\prime}=\$ B \$ 7^{*} \$ B \$ 33^{*} \$ B \$ 4^{*} \$ B \$ 5^{*} \$ B \$ 6^{*} \$ B \$ 8^{*} E 34^{*} R$ & $=\$ 8 \$ 7^{*}$ \$B\$8*\$C34"Relocation!E34"\$B\$35/\$D\$35 \\
\hline 36 & $=\mathbf{R}$ & $=\$ B \$ 7^{*} \$ B \$ 8^{*} \$ C 36^{*}$ Relocation!E36*\$B $\$ 35 / \$ D \$ 35$ \\
\hline 37 & $=\mathbf{R}$ & $=\$ B \$ 7^{*} \$ B S 8^{*}$ \$C $37^{*}$ Relocation!E37*\$B\$35/\$D\$3 \\
\hline 38 & $=\mathbf{R}$ & $=\$ B S 7^{*}$ SBS8'SC38*Relocation!E38"\$B\$35/\$DS35 \\
\hline 39 & $=\mathbf{R}$ & $=\$ B \$ 7^{*} \$ B \$ 8^{*} \$ C 39^{*}$ Relocation!E39*\$BS35/\$D \$35 \\
\hline 40 & $=\mathbf{R}$ & $=\$ B \$ 7^{*} \$ B \$ 8^{*} \$ C 40^{*}$ Relocation!E40*\$BS35/SD\$35 \\
\hline 41 & $=\mathbf{R}$ & 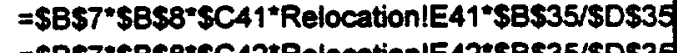 \\
\hline 42 & $=\mathbf{R}$ & 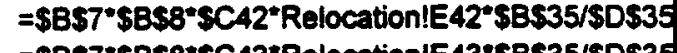 \\
\hline 43 & $=\mathbf{R}$ & $=\$ B \$ 77^{*} \$ B \$ 8^{*}$ SC43"RelocationIE43"\$B\$35/\$D \$35 \\
\hline 44 & $=R$ & $=\$ 8 \$ 7^{*}$ \$BS8*\$C44*Relocation!E44*\$B\$35/\$D\$35 \\
\hline 45 & $=\mathbf{R}$ & $=\$ 8 \$ 7^{*} \$ 8 \$ 8^{*}$ SC45"Relocation!E45"\$B\$35/\$D\$35 \\
\hline 46 & & \\
\hline 47 & $T=S U M Y$ & UM(H10:H46) \\
\hline
\end{tabular}




\begin{tabular}{|c|c|c|c|}
\hline & $\bar{A}$ & $\mathrm{~J}$ & $K$ \\
\hline 9 & & Skin Dose (mrem per uCi/m^2) & $\begin{array}{l}\text { BS Dose } \\
\text { (mrem per } \\
u C l / m^{\wedge} 2 \text { ) }\end{array}$ \\
\hline 10 & Am-241 & $7.73 E-02$ & $4.69 E+02$ \\
\hline 11 & Am-243 & 2.88E-03 & $1.44 E+01$ \\
\hline 12 & Ba-133. & $8.79 E-12$ & $5.58 E-14$ \\
\hline 13 & Ce-144 & 1.52E-05 & $9.75 E-07$ \\
\hline 14 & C -249 & $1.70 E-04$ & $3.98 E-01$ \\
\hline 15 & C & $2.08 E-03$ & $2.38 \mathrm{E}+01$ \\
\hline 16 & $\mathrm{Cm}-244$ & $3.74 E-03$ & $2.91 E+02$ \\
\hline 17 & $\mathrm{Cm}-246$ & $1.64 E-04$ & $1.61 E+01$ \\
\hline 18 & Co-60 & $6.16 E+01$ & $1.06 E-01$ \\
\hline 19 & Cs-137 & $9.37 E+\infty 0$ & $1.03 E-01$ \\
\hline 20 & H-3 & $0.00 E+\infty 0$ & $0.00 E+\infty 0$ \\
\hline 21 & Np-237 & $8.89 E-05$ & $1.16 E+\infty$ \\
\hline 22 & $P m-147$ & $3.51 E-12$ & $5.52 \mathrm{E}-09$ \\
\hline 23 & Pu-238 & $1.11 E-02$ & $5.00 E+02$ \\
\hline 24 & Pu-239 & $7.27 E-03$ & $7.20 E+02$ \\
\hline 25 & Pu mix-239/241 & $0.00 E+00$ & $0.00 E+00$ \\
\hline 26 & Pu-240 & 4.06E-03 & $1.64 E+02$ \\
\hline 27 & Pu-241 & $0.00 E+00$ & $1.63 E+02$ \\
\hline 28 & Pu pure-242 & $2.33 E-05$ & $1.01 E+00$ \\
\hline 29 & Sr-90 & $5.72 E+\infty 0$ & $9.10 E-01$ \\
\hline 30 & Te-99 & 7.81E-18 & 1.57E-16 \\
\hline 31 & Th-232 & 2.63E-07 & 2.32E-01 \\
\hline 32 & ]T-204 & $3.41 E-08$ & 4.07E-12 \\
\hline 33 & U-235 & 1.14E-05 & $1.05 E-05$ \\
\hline 34 & ]U-238 & $3.20 E-05$ & $7.51 E-04$ \\
\hline 35 & Total & & \\
\hline 36 & | $8 a-137 m$ & 6.37E+01 & \\
\hline 37 & JY-90 & $3.39 E+02$ & \\
\hline 38 & |Pr-144 & 7.93E-03 & \\
\hline 39 & Pa-233 & $3.87 E-04$ & \\
\hline 40 & Th-234 & $1.07 E-04$ & \\
\hline 41 & |Pa-234 & $2.44 E-02$ & \\
\hline 42 & Np-239 & $8.09 E-03$ & \\
\hline 43 & Th-231 & $3.22 E-06$ & \\
\hline 44 & Ra-228 & 4.35E-13 & \\
\hline 45 & |AC-228 & $2.73 E-04$ & \\
\hline 46 & & & \\
\hline 47 & 1 Total & $4.80 \mathrm{E}+02$ & $2.37 E+08$ \\
\hline
\end{tabular}




\begin{tabular}{|c|c|c|}
\hline It & $\mathrm{J}$ & $\bar{K}$ \\
\hline 9 & Skin Dose (mrem per uCi/m^2) & BS Dose (mrem per uCl/m^2) \\
\hline 10 & $E^{\prime}=\$ 8 \$ 7^{*} \$ 13 \$ 8^{*} \$ C 10^{*}$ Relocation $\mid \$ F 10^{*} \$ B \$ 35 / \$ D \$ 35$ & $=\$ B \$ 7^{*} \$ B \$ 3 * \$ B \$ 4^{*} \$ B \$ 5^{*} \$ B \$ 6^{*} \$ B \$ 8^{*}$ \$E $10^{*}$ Relocation $\$$ \$J10 \\
\hline & 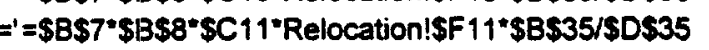 & $=\$ B \$ 7^{*} \$ B \$ 3^{*} \$ B \$ 4^{*} \$ B \$ 5^{*} \$ B \$ 6^{*} \$ B S 8^{*}$ \$E $11^{*}$ Relocation!\$J11 \\
\hline $12=$ & $=^{\prime}=\$ B \$ 7^{*} \$ B \$ 8^{*} \$ C 12^{*}$ Relocation $\$$ \$F 12*\$B\$35/\$D\$35 & $=\$ B \$ 7^{*} \$ B \$ 3^{*} \$ B \$ 4^{*} \$ B \$ 5^{*} \$ B S 66^{*} \$ B \$ 8^{*} \$ E 12^{*}$ Relocation! $\$ 112$ \\
\hline 13 & $E^{\prime}=\$ B \$ 7^{*} \$ 18 \$ 8^{*} \$ C 13^{*}$ Relocation $\$$ \$F 13*\$B\$35/\$DS35 & 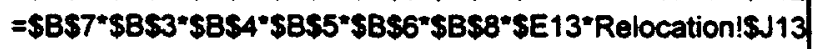 \\
\hline $14=$ & $=^{\prime}=\$ B \$ 7^{*} \$ 1 B \$ 8^{*}$ \$C14"Relocation!SF14"\$B\$35/\$D\$35 & $=\$ B \$ 7^{*}$ \$BS3*\$BS4*\$BS5*\$BS6*\$BS8*\$E14* Relocation!\$J14 \\
\hline$\frac{15}{19}=$ & $=1=$ \$BS7" SBS8+\$C15"Relocation!SF15" \$B\$35/\$D\$35 & 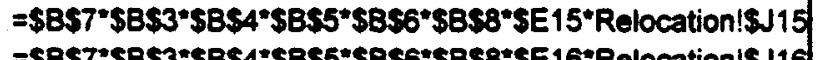 \\
\hline$\frac{16}{17}=$ & 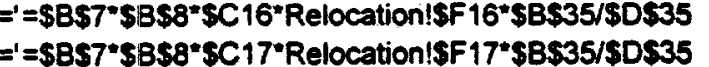 & 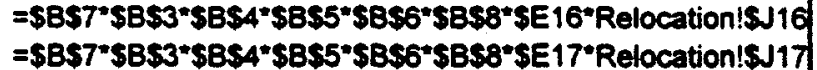 \\
\hline $18=$ & $='=\$ B \$ 7^{*}$ SBS8* SC $18^{*}$ Relocation!\$F $18^{*}$ \$B \$35/\$D\$35 & 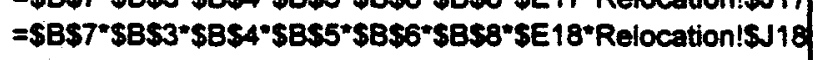 \\
\hline 19 & $={ }^{\prime}=$ \$B\$7* \$B\$8*\$C19*Relocation!\$F19*\$B \$35/\$D\$35 & 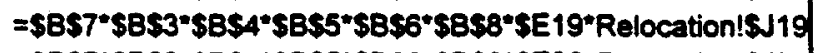 \\
\hline 20 & $==^{\prime}=\$ B \$ 7^{*} \$ B \$ 8 * \$ C 20^{*}$ Relocation!\$F20*\$B\$35/\$D\$35 & $=\$ B \$ 7^{*} \$ B \$ 33^{*} \$ B S 4^{*} \$ B \$ 55^{*} \$ B \$ 6^{*} \$ B \$ 8^{*} \$ E 20^{*}$ Relocation!\$J20 \\
\hline$\frac{21}{20}=$ & $='=$ SB\$7* \$B\$8*\$C21*Relocation!SF21"\$B\$35/\$D\$35 & 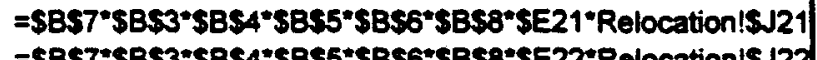 \\
\hline$\frac{22}{23}=$ & 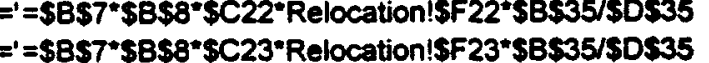 & 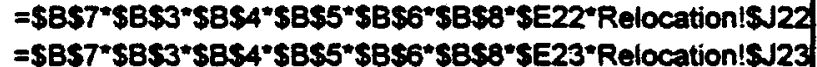 \\
\hline 24 & $=^{\prime}=\$ 8 \$ 7^{*} \$ B \$ 8^{*}$ \$C24"Relocation!SF24*\$B\$35/SD\$35 & \\
\hline 25 & $=^{\prime}=\$ B \$ 7 *$ \$BS8*\$C25*Relocation!\$F25*\$B\$35/\$D\$35 & $=\$ 8 \$ 7^{*} \$ B \$ 3 * \$ B \$ A^{*} \$ B \$ 5^{*} \$ B \$ 6 * \$ B \$ 8^{*} \$ E 25 "$ Relocation!\$J25 \\
\hline $26=$ & $=^{\prime}=\$ B \$ 7^{*} \$ B \$ 8^{*} \$ C 26 *$ Relocation!\$F26"\$B\$35/\$D\$35 & 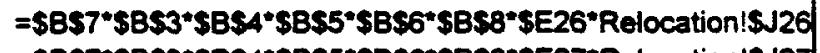 \\
\hline $27:$ & $=^{\prime}=\$ B \$ 7^{*} \$ B \$ 88^{*} \$ 27^{*}$ Relocation!\$F27*\$B\$35/\$D \$35 & 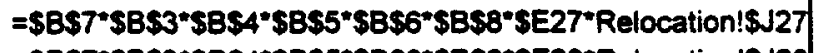 \\
\hline$\frac{28}{29}=$ & $='=\$ B \$ 7 * \$ B \$ 8 *$ * $=28 *$ Relocation!\$F28*\$B\$35/\$D\$35 & 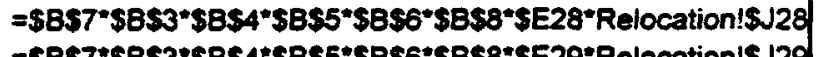 \\
\hline$\frac{29}{30}$ & 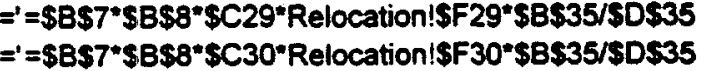 & 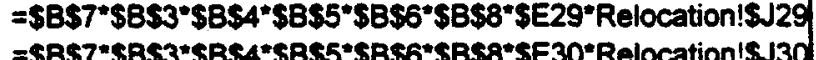 \\
\hline 31 & 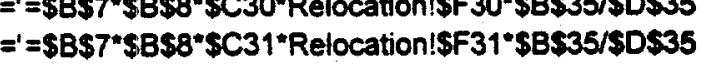 & \\
\hline 32 & $={ }^{\prime}=$ \$B\$7" $\$$ B\$8*\$C32"Relocation!\$F32*\$B\$35/\$D\$35 & 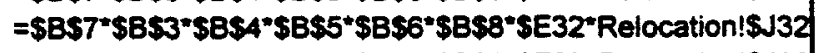 \\
\hline 33 & $==^{\prime}=\$ B \$ 7^{*} \$ B \$ 8^{*} \$ C 33^{*}$ Relocation!\$F33*\$BS35/\$D\$35 & $=\$ B \$ 7^{*} \$ B \$ 3^{*} \$ B \$ A^{*} \$ B \$ 55^{*} \$ B \$ 6^{*} \$ B \$ 8^{*} \$ E 33^{*}$ Relocation!SJ33 \\
\hline 34 & $z^{\prime}=\$ B \$ 7^{*} \$ B \$ 8^{*} \$ C 34^{*}$ Relocation!\$F34*\$B\$35/\$D\$35 & $=\$ B \$ 7^{*} \$ B \$ 33^{*} \$ B \$ 4^{*} \$ B \$ 55^{*} \$ B \$ 6^{*} \$ B \$ 8^{*} \$ E 34^{*}$ Relocation!\$J34 \\
\hline 35 & 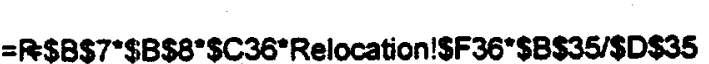 & \\
\hline 37 & 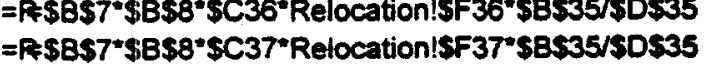 & \\
\hline 38 & 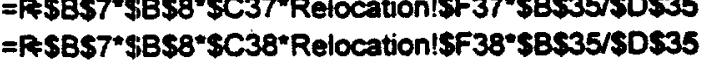 & \\
\hline 39 & $=$ PABB $\$ 7^{*} \$ B S 8 *$ SC $39^{*}$ Relocation!\$F39*\$B\$35/SD $\$ 35$ & \\
\hline 40 & $=R \$ B S 7^{*} \$ B S 8^{*}$ SC40*Relocation!\$F 40"\$B\$35/SD\$35 & \\
\hline 41 & $=$ A $\$ B S 7^{*} \$ B S 8 * \$ C 41^{*}$ Relocation!\$F41"\$BS35/SDS35 & \\
\hline 42 & $=R * \$ B \$ 7 * \$ B \$ 8 *$ \$CA2*Relocation!SF 42*\$B\$35/\$D\$35 & \\
\hline 43 & $=$ R*\$B\$7*\$BSB*\$C43*Relocation!\$F43*\$B\$35/\$D\$35 & \\
\hline 44 & $=$ R \$B\$7"\$B\$8"\$C44"Relocation!SF44"\$B\$35/\$OS35 & \\
\hline 45 & $=R \neq \$ B \$ 7^{*} \$ B \$ 88^{*} \$ C 45^{*}$ Relocation!SF45*\$BS35/SD\$35 & \\
\hline 46 & & \\
\hline 47 & $110: \sqrt{46)}$ & UM(K10:K46) \\
\hline
\end{tabular}




\begin{tabular}{|c|c|c|}
\hline & $F$ & \multicolumn{1}{c|}{ G } \\
\hline 52 & $9.60 E+00$ & DRL (uCi/m²) - PAG/(CEDE+External) \\
\hline 53 & $8.23 E+00$ & DRL (UCi/m^2) - PAG/(BS Dose + External) \\
\hline 54 & $2.08 E+02$ & DRL (uCi/m^2) - PAG/(Skin Dose) \\
\hline
\end{tabular}




\begin{tabular}{|c|c|c|}
\hline & $F$ & $\mathbf{G}$ \\
\hline 52 & $=H 3 /(647+H 47)$ & DRL (uCi/m^2) - PAG/(CEDE+Extemal) \\
\hline 53 & $=H 4 /(K 47+H 47)$ & DRL (uCi/m^2) - PAG/(BS Dose + External) \\
\hline 54 & $=H 5 / J 47$ & DRL (uCi/m^2) - PAG/(Skin Dose) \\
\hline
\end{tabular}




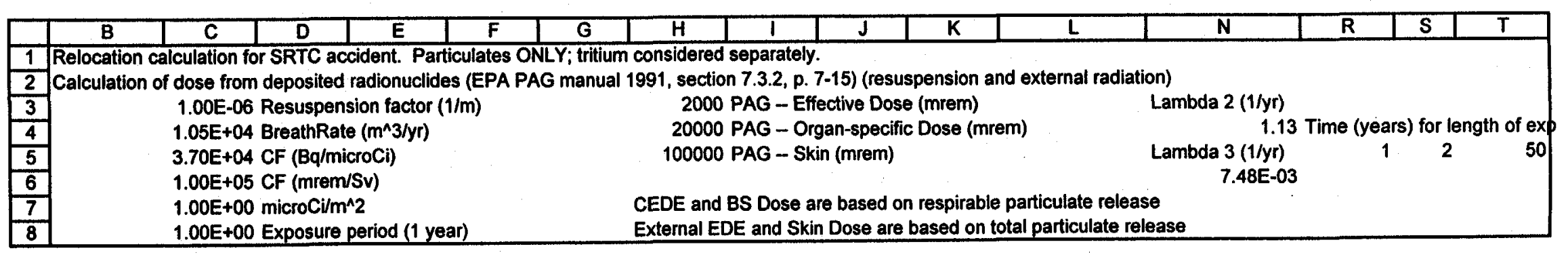




\begin{tabular}{|c|c|c|c|c|c|c|c|c|c|}
\hline & B & $\mathrm{C}$ & $\bar{D}$ & $\bar{H}$ & 1 & $N$ & $\overline{\mathbf{R}}$ & $\bar{S}$ & $T$ \\
\hline 1 & \multirow{2}{*}{\multicolumn{9}{|c|}{$\begin{array}{l}\text { Relocation calculation for S } \\
\text { Calculation of dose from de }\end{array}$}} \\
\hline 2 & & & & & & & & & \\
\hline 3 & & $=$ Relo_HIB3 & $=$ Relo_HIC3 & 2000 & \multicolumn{2}{|c|}{ PAG - Effective DoseLambda 2 (1/yr) } & \multirow{2}{*}{\multicolumn{2}{|c|}{ Time (years) for length }} & \\
\hline 4 & & $=$ Relo_H!B4 & $=$ Relo_H!C4 & 20000 & PAG - Organ-specifi & ic1.13 & & & \\
\hline 5 & & $=$ Relo_H!B5 & $=$ Relo_H!C5 & 100000 & PAG - Skin (mrem) & Lambda 3 (1/yr) & 1 & 2 & 50 \\
\hline 6 & & $=$ Relo_HIB6 & $=$ Relo_HIC6 & & & 0.00748 & & & \\
\hline 7 & & $=$ Relo_HIB7 & $=$ Relo_HIC7 & CEDE and BS Dose are & & & & & \\
\hline 8 & & $=$ Relo_HIB8 & $=$ Relo_H!C8 & External EDE and Skin & & & & & \\
\hline
\end{tabular}




\begin{tabular}{|c|c|c|c|}
\hline & B & $\mathrm{C}$ & $\bar{D}$ \\
\hline 9 & Nuclide & Total Release (Ci) & $\begin{array}{c}\text { Activity fraction } \\
\text { (Total) }\end{array}$ \\
\hline 10 & Am-241 & $3.23 E-02$ & $1.09 E-02$ \\
\hline 11 & Am-243 & $7.26 E-04$ & 2.45E-04 \\
\hline 12 & $B a-133$ & $4.04 E-13$ & $1.36 E-13$ \\
\hline 13 & $\mathrm{Ce}-144$ & 1.42E-05 & 4.8@E-06 \\
\hline 14 & Cf-249 & $9.53 E-06$ & $3.22 E-06$ \\
\hline 15 & $C f-252$ & 1.19E-02 & 4.03E-03 \\
\hline 16 & $\mathrm{Cm}-244$ & $1.53 E-02$ & $5.15 E-03$ \\
\hline 17 & $\mathrm{Cm}-246$ & 7.50E-04 & 2.53E-04 \\
\hline 18 & Co-60 & 5.40E-01 & 1.82E-01 \\
\hline 19 & Cs-137 & 8.91E-01 & 3.01E-01 \\
\hline 20 & $\mathrm{H}-3$ & $0.00 E+\infty 0$ & $0.00 \mathrm{E}+00$ \\
\hline 21 & $\mathrm{~Np}-237$ & 3.15E-05 & $1.06 E-05$ \\
\hline 22 & $\mathrm{Pm}-147$ & $1.89 E-08$ & $6.38 E-09$ \\
\hline 23 & Pu-238 & 4.15E-02 & 1.40E-02 \\
\hline 24 & Pu-239 & $6.94 E-02$ & 2.34E-02 \\
\hline 25 & Pu mix-239/241 & $0.00 E+\infty 0$ & $0.00 \mathrm{E}+00$ \\
\hline 26 & $\mathrm{Pu}-240$ & 1.59E-02 & 5.37E-03 \\
\hline 27 & $P u-241$ & 4.58E-01 & $1.55 \mathrm{E}-01$ \\
\hline 28 & Pu pure-242 & 1.10E-04 & $3.70 E-05$ \\
\hline 29 & Sr-90 & 8.84E-01 & 2.99E-01 \\
\hline 30 & Tc-99 & $2.70 E-13$ & $9.12 E-14$ \\
\hline 31 & Th-232 & $1.45 E-06$ & 4.90E-07 \\
\hline 32 & $\pi /-204$ & $6.75 E-10$ & 2.28E-10 \\
\hline 33 & U-235 & $1.34 E-06$ & 4.52E-07 \\
\hline 34 & U-238 & $1.62 E-04$ & 5.45E-05 \\
\hline 35 & Total & $2.96 \mathrm{E}+00$ & $1.00 E+00$ \\
\hline 36 & $\mathrm{Ba}-137 \mathrm{~m}$ & 8.91E-01 & 3.01E-01 \\
\hline 37 & $Y-90$ & 8.84E-01 & 2.99E-01 \\
\hline 38 & Pr-144 & $1.42 E-05$ & $4.80 E-06$ \\
\hline 39 & $\mathrm{~Pa}-233$ & 3.15E-05 & $1.06 \mathrm{E}-05$ \\
\hline 40 & Th-234 & 1.62E-04 & $5.45 E-05$ \\
\hline 41 & Pa-234 & $1.62 E-04$ & 5.45E-05 \\
\hline 42 & $\mathrm{~Np}-239$ & 7.26E-04 & 2.45E-04 \\
\hline 43 & Th-231 & 1.34E-06 & 4.52E-07 \\
\hline 44 & Ra-228 & $1.45 E-06$ & 4.90E-07 \\
\hline 45 & Ac-228 & $1.45 E-06$ & 4.90E-07 \\
\hline
\end{tabular}




\begin{tabular}{|c|c|c|c|}
\hline & $\bar{B}$ & $\mathrm{C}$ & D \\
\hline 9 & Nuclide & Total Release (Ci) & Activity fraction (Total) \\
\hline 10 & Am-241 & ='C:IEP_relocationYSourceSRTC.xIs]resp sums'!\$H10 & $=\mathrm{C} 10 / \$ \mathrm{C} \$ 35$ \\
\hline 11 & Am-243 & ='C:IEP_relocation \SourceSRTC.x|s]resp sums'!\$H11 & $=C 11 / \$ C \$ 35$ \\
\hline 12 & Ba-133 & $=' C:$ IEP_relocation VSourceSRTC.xls]resp sums'!\$H12 & $=\mathrm{C} 12 / \$ \mathrm{C} \$ 35$ \\
\hline 13 & Ce-144 & $=' C:$ IEP_relocation USourceSRTC.x|s]resp sums'|\$H13 & $=\mathrm{C} 13 / \$ \operatorname{CS} 35$ \\
\hline 14 & Cf-249 & ='C:IEP_relocation\SourceSRTC.xIs]resp sums'!\$H14 & $=\mathrm{C} 14 / \$ \mathrm{C} \$ 35$ \\
\hline 15 & Cf-252 & ='C:IEP_relocationUSourceSRTC.xIs]resp sums'!\$H15 & $=C 15 / \$ C \$ 35$ \\
\hline 16 & $\mathrm{Cm}-244$ & $=' C:$ IEP_relocation YSourceSRTC.xls\}resp sums'!\$H16 & $=C 16 / \$ C \$ 35$ \\
\hline 17 & $\mathrm{Cm}-246$ & ='C:IEP_relocation〈SourceSRTC.xis]resp sums'!\$H17 & $=C 17 / \$ C \$ 35$ \\
\hline 18 & Co-60 & $=$ ='C:LEP_relocation VSourceSRTC.xIs]resp sums'!\$H18 & $=\mathrm{C} 18 / \$ C \$ 35$ \\
\hline 19 & Cs-137 & ='C:IEP_relocationYSourceSRTC.xls]resp sums'!\$H19 & $=C 19 / \$ C \$ 35$ \\
\hline 20 & $\mathrm{H}-3$ & 0 & $=\mathrm{C} 20 / \$ C \$ 35$ \\
\hline 21 & Np-237 & $={ }^{\prime} C:$ IEP_relocationYSourceSRTC.xls]resp sums'!\$H21 & $=\mathrm{C} 21 / \$ \mathrm{C} \$ 35$ \\
\hline 22 & $P m-147$ & $=' C:$ LEP_reiocationYSSourceSRTC.xIs]resp sums'!\$H22 & $=C 22 / \$ C \$ 35$ \\
\hline 23 & Pu-238 & $=$ 'C:IEP_relocation $[$ SourceSRTC.xIs]resp sums'!\$H23 & $=\mathrm{C} 23 / \$ C \$ 35$ \\
\hline 24 & Pu-239 & $=$ ='C:IEP_relocationUSourceSRTC.xIs]resp sums'!\$H24 & $=\mathrm{C} 24 / \$ \mathrm{C} \$ 35$ \\
\hline 25 & Pu mix-239/241 & $=$ ='C:IEP_relocation\SourceSRTC.xls]resp sums'!\$H25 & $=C 25 / \$ C \$ 35$ \\
\hline 26 & $P u-240$ & $={ }^{\prime} C$ :IEP_relocationYSSourceSRTC.xIs]resp sums'!\$H26 & $=\mathrm{C} 26 / \$ C \$ 35$ \\
\hline 27 & Pu-241 & ='C:IEP_relocationYSourceSRTC.xis]resp sums'!\$H27 & $=\mathrm{C} 27 / \$ C \$ 35$ \\
\hline 28 & Pu pure-242 & 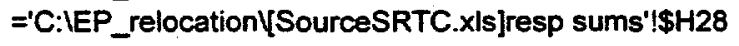 & $=C 28 / \$ C \$ 35$ \\
\hline 29 & Sr-90 & ='C:IEP_relocation)[SourceSRTC.xIs]resp sums'!\$H29 & $=C 29 / \$ C \$ 35$ \\
\hline 30 & Tc-99 & ='C:IEP_relocationYSourceSRTC.xIs]resp sums'!\$H30 & $=C 30 / \$ C \$ 35$ \\
\hline 31 & Th-232 & ='C:IEP_relocationYSourceSRTC.xis]resp sums'!\$H31 & $=\mathrm{C} 31 / \$ \mathrm{C} \$ 35$ \\
\hline 32 & TI-204 & ='C:IEP_relocationYSourceSRTC.xis]resp sums'!\$H32 & $=C 32 / \$ C \$ 35$ \\
\hline 33 & U-235 & ='C:IEP_relocationViSourceSRTC.xls]resp sums'!\$H33 & $=\mathrm{C} 33 / \$ C \$ 35$ \\
\hline 34 & $U-238$ & ='C:IEP_relocationYSourceSRTC.xls]resp sums'!\$H34 & $=\mathrm{C} 34 / \$ C \$ 35$ \\
\hline 35 & Total & $=S U M(C 10: C 34)$ & $=$ SUM(D10:D34) \\
\hline 36 & Ba-137m & $=\mathrm{C} 19$ & $=\mathrm{D} 19$ \\
\hline 37 & $Y-90$ & $=C 29$ & $=\mathrm{D} 29$ \\
\hline 38 & Pr-144 & $=\mathrm{C} 13$ & $=013$ \\
\hline 39 & $\mathrm{~Pa}-233$ & $=C 21$ & $=\mathrm{D} 21$ \\
\hline 40 & Th-234 & $=\mathrm{C} 34$ & $=\mathrm{D} 34$ \\
\hline 41 & Pa-234 & $=\mathrm{C} 34$ & $=\mathrm{D} 34$ \\
\hline 42 & Np-239 & $=C 11$ & $=D 11$ \\
\hline 43 & Th-231 & $=\mathrm{C} 33$ & $=033$ \\
\hline 44 & Fia-228 & $=C 31$ & $=\mathrm{D} 31$ \\
\hline 45 & A.C-228 & $=C 31$ & $=\mathrm{D} 31$ \\
\hline
\end{tabular}




\begin{tabular}{|c|c|c|c|}
\hline & B & $\bar{E}$ & $\bar{F}$ \\
\hline 9 & Nuclide & Respirable Release (Ci) & $\begin{array}{c}\text { Activity fraction } \\
\text { (Resp) }\end{array}$ \\
\hline 10 & Am-241 & 1.49E-02 & $5.56 E-03$ \\
\hline 11 & Am-243 & 4.55E-04 & 1.70 E-04 \\
\hline 12 & $B a-133$ & $4.04 E-13$ & $1.51 E-13$ \\
\hline 13 & $\mathrm{Ce}-144$ & $1.42 E-05$ & 5.32E-06 \\
\hline 14 & Cf-249 & $8.24 E-06$ & 3.08E-06 \\
\hline 15 & $\mathrm{Cf}-252$ & $1.19 E-02$ & 4.47E-03 \\
\hline 16 & $\mathrm{Cm}-244$ & 1.71E-02 & $6.40 E-03$ \\
\hline 17 & $\mathrm{Cm}-246$ & $4.99 E-04$ & $1.87 E-04$ \\
\hline 18 & Co-60 & 5.40E-01 & 2.02E-01 \\
\hline 19 & Cs- 137 & 8.91E-01 & $3.34 E-01$ \\
\hline 20 & $\mathrm{H}-3$ & $0.00 E+00$ & $0.00 E+00$ \\
\hline 21 & $\mathrm{~Np}-237$ & 2.44E-05 & $9.14 E-06$ \\
\hline 22 & $\mathrm{Pm}-147$ & $1.89 E-08$ & 7.07E-09 \\
\hline 23 & Pu-238 & $1.81 E-02$ & 6.77E-03 \\
\hline 24 & Pu-239 & 2.35E-02 & 8.78E-03 \\
\hline 25 & Pu mix-239 & $0.00 E+00$ & $0.00 E+00$ \\
\hline 26 & Pu-240 & 5.34E-03 & $2.00 E-03$ \\
\hline 27 & Pu-241 & 2.66E-01 & 9.97E-02 \\
\hline 28 & Pu pure-24 & 3.47E-05 & $1.30 E-05$ \\
\hline 29 & Sr-90 & $8.83 E-01$ & 3.30E-01 \\
\hline 30 & Tc-99 & $2.70 E-13$ & $1.01 E-13$ \\
\hline 31 & Th-232 & 1.44E-06 & 5.38E-07 \\
\hline 32 & TI-204 & $6.75 E-10$ & 2.53E-10 \\
\hline 33 & U-235 & 6.88E-07 & 2.57E-07 \\
\hline 34 & U-238 & 5.11E-05 & 1.91E-05 \\
\hline 35 & Total & 2.67E+00 & $1.00 E+\infty 0$ \\
\hline 36 & $\mathrm{Ba}-137 \mathrm{~m}$ & $8.91 E-01$ & 3.34E-01 \\
\hline 37 & $Y-90$ & 8.83E-01 & $3.30 E-01$ \\
\hline 38 & Pr-144 & $1.42 E-05$ & 5.32E-06 \\
\hline 39 & Pa-233 & 2.44E-05 & 9.14E-06 \\
\hline 40 & Th-234 & 5.11E-05 & $1.91 \mathrm{E}-05$ \\
\hline 41 & $\mathrm{~Pa}-234$ & 5.11E-05 & $1.91 E-05$ \\
\hline 42 & $\mathrm{~Np}-239$ & $4.55 E-04$ & $1.70 E-04$ \\
\hline 43 & Th-231 & $6.88 E-07$ & 2.57E-07 \\
\hline 44 & Ra-228 & $1.44 E-06$ & 5.38E-07 \\
\hline 45 & $A C-228$ & $1.44 E-06$ & 5.38E-07 \\
\hline
\end{tabular}




\begin{tabular}{|c|c|c|c|}
\hline & $\mathbf{B}$ & $\mathbf{E}$ & $\bar{F}$ \\
\hline 9 & Nuclide & Respirable Release (Ci) & Activity fraction (Resp) \\
\hline 10 & Am-241 & $=' C$ :IEP_relocationVSourceSRTC.x|s]resp sums'|\$F10 & $=E 10 / \$ E \$ 35$ \\
\hline 11 & Am-243 & $={ }^{\prime} C:$ IEP_relocationVSourceSRTC.xIs]resp sums'!\$F11 & $=\mathrm{E} 11 / \$ \mathrm{E} \$ 35$ \\
\hline 12 & Ba-133 & $=$ 'C:IEP_relocationYSourceSRTC.xIs]resp sums'!\$F12 & $=E 12 / \$ E \$ 35$ \\
\hline 13 & $\mathrm{Ce}-144$ & ='C:IEP_relocationYSourceSRTC.xIs]resp sums'!\$F13 & $=E 13 / \$ E \$ 35$ \\
\hline 14 & $\mathrm{Cf}-249$ & $=' C$ :IEP_relocationVSourceSRTC.xis]resp sums'!\$F14 & $=E 14 / \$ E \$ 35$ \\
\hline 15 & Cf-252 & $=' C:$ IEP_relocationUSourceSRTC.x|s]resp sums'!\$F15 & $=E 15 / \$ E \$ 35$ \\
\hline 16 & $\mathrm{Cm}-244$ & $=' C: I E P \_$relocationVSourceSRTC.x|s]resp sums' $\$$ F 16 & $=E 16 / \$ E \$ 35$ \\
\hline 17 & $\mathrm{Cm}-246$ & ='C:IEP_relocationVSSourceSRTC.xIs]resp sums'!\$F17 & $=E 17 / \$ E \$ 35$ \\
\hline 18 & Co-60 & ='C:IEP_relocationYSourceSRTC.xls]resp sums'!\$F18 & $=E 18 / \$ E \$ 35$ \\
\hline 19 & Cs-137 & ='C:IEP_relocationYSourceSRTC.xIs]resp sums'!\$F19 & $=E 19 / \$ E \$ 35$ \\
\hline 20 & $\mathrm{H}-3$ & 0 & $=E 20 / \$ E \$ 35$ \\
\hline 21 & Np-237 & $=$ 'C:IEP_relocationVSourceSRTC.xls]resp sums'!\$F21 & $=E 21 / \$ E \$ 35$ \\
\hline 22 & Pm-147 & ='C:IEP_relocationYSourceSRTC.xls]resp sums'!\$F22 & $=E 22 / \$ E \$ 35$ \\
\hline 23 & Pu-238 & ='C:IEP_relocationVSourceSRTC.xIs]resp sums'!\$F23 & $=E 23 / \$ E \$ 35$ \\
\hline 24 & Pu-239 & $=$ 'C:IEP_relocationVSourceSRTC.x|s]resp sums'!\$F24 & $=E 24 / \$ E \$ 35$ \\
\hline 25 & Pu mix-239/241 & ='C:IEP_relocationYSourceSRTC.xIs]resp sums'!\$F25 & $=E 25 / \$ E \$ 35$ \\
\hline 26 & Pu-240 & ='C:IEP_relocationYSourceSRTC.x|s]resp sums'!\$F26 & $=E 26 / \$ E \$ 35$ \\
\hline 27 & Pu-241 & ='C:IEP_relocationYSourceSRTC.xls]resp sums'!\$F27 & $=E 27 / \$ E \$ 35$ \\
\hline 28 & Pu pure-242 & ='C:IEP_relocationYSourceSRTC.xls]resp sums'!\$F28 & $=E 28 / \$ E \$ 35$ \\
\hline 29 & Sr-90 & ='C:IEP_relocationYSourceSRTC.xls]resp sums'!\$F29 & $=E 29 / \$ E \$ 35$ \\
\hline 30 & Tc-99 & ='C:IEP_relocationYSourceSRTC.xls]resp sums'!\$F30 & $=E 30 / \$ E \$ 35$ \\
\hline 31 & Th-232 & ='C:IEP_relocationYSourceSRTC.xls]resp sums'!\$F31 & $=E 31 / \$ E \$ 35$ \\
\hline 32 & TI-204 & ='C:IEP_relocationYSourceSRTC.xls]resp sums'!\$F32 & $=E 32 / \$ E \$ 35$ \\
\hline 33 & $\mathrm{U}-235$ & ='C:IEP_relocationYSourceSRTC.xls]resp sums'!\$F33 & $=E 33 / \$ E \$ 35$ \\
\hline 34 & U-238 & ='C:IEP_relocationYSourceSRTC.xls]resp sums'!\$F34 & $=E 34 / \$ E \$ 35$ \\
\hline 35 & Total & =SUM(E10:E34) & $=$ SUM(F10:F34) \\
\hline 36 & $8 a-137 m$ & $=E 19$ & $=F 19$ \\
\hline 37 & $Y-90$ & $=E 29$ & $=F 29$ \\
\hline 38 & $\operatorname{Pr}-144$ & $=E 13$ & $=F 13$ \\
\hline 39 & $\mathrm{~Pa}-233$ & $=E 21$ & $=\mathbf{F} 21$ \\
\hline 40 & Th-234 & $=E 34$ & $=$ F34 \\
\hline 41 & Pa-234 & $=E 34$ & $=F 34$ \\
\hline 42 & $\mathrm{~Np}-239$ & $=E 11$ & $=F 11$ \\
\hline 43 & Th-231 & $=E 33$ & $=F 33$ \\
\hline 44 & $R a-228$ & $=E 31$ & $=F 31$ \\
\hline 45 & Ac-228 & $=E 31$ & $=F 31$ \\
\hline
\end{tabular}




\begin{tabular}{|c|c|c|c|}
\hline & $\bar{B}$ & $\mathrm{H}$ & I \\
\hline & & $\begin{array}{c}\text { CEDE } \\
\text { (mrem per }\end{array}$ & $\begin{array}{l}\text { External } \\
\text { EDE } \\
\text { (mrem per }\end{array}$ \\
\hline 9 & Nuclide & $\left.\mathrm{uCl} / \mathrm{m}^{\wedge} 2\right)$ & $\left.u C i / m^{\wedge} 2\right)$ \\
\hline 10 & Am-241 & $1.93 E+01$ & 2.70E-02 \\
\hline 11 & Am-243 & 5.87E-01 & $1.34 E-03$ \\
\hline 12 & $B a-133$ & $8.96 E-15$ & $4.58 E-12$ \\
\hline 13 & $\mathrm{Ce}-144$ & 1.07E-05 & 5.92E-06 \\
\hline 14 & Cf-249 & 1.39E-02. & $9.36 E-05$ \\
\hline 15 & $C f-252$ & $4.89 E+00$ & $1.88 E-04$ \\
\hline 16 & $\mathrm{Cm}-244$ & $1.22 \mathrm{E}+01$ & 3.47E-04 \\
\hline 17 & $\mathrm{Cm}-246$ & 6.60E-01 & $1.54 E-05$ \\
\hline 18 & Co-60 & $3.26 E-01$ & $3.22 E+01$ \\
\hline 19 & Cs-137 & 8.26E-02 & $0.00 E+00$ \\
\hline 20 & H-3 & & \\
\hline 21 & $\mathrm{~Np}-237$ & $3.87 E-02$ & 2.85E-05 \\
\hline 22 & $P m-147$ & 1.93E-09 & $1.92 E-12$ \\
\hline 23 & Pu-238 & $2.07 E+01$ & $9.90 E-04$ \\
\hline 24 & Pu-239 & $2.95 E+01$ & 7.32E-04 \\
\hline 25 & Pu mix-239/241 & & \\
\hline 26 & $P u-240$ & $6.72 E+00$ & $3.64 E-04$ \\
\hline 27 & $P u-241$ & $6.29 E+00$ & $0.00 E+00$ \\
\hline 28 & Pu pure-242 & 4.18E-02 & 2.09E-06 \\
\hline 29 & Sr-90 & $3.32 E+00$ & $0.00 E+00$ \\
\hline 30 & TC-99 & $6.59 E-15$ & $4.72 E-18$ \\
\hline 31 & Th-232 & 6.91E-03 & $2.70 E-08$ \\
\hline 32 & TI-204 & $4.39 E-12$ & $2.26 \mathrm{E}-11$ \\
\hline 33 & U-235 & $2.48 E-04$ & $6.39 E-06$ \\
\hline 34 & U-238 & 1.77E-02 & 2.91E-06 \\
\hline 35 & Total & & \\
\hline 36 & $\mathrm{Ba}-137 \mathrm{~m}$ & & 1.43E-04 \\
\hline 37 & $Y-90$ & & $0.00 E+00$ \\
\hline 38 & Pr-144 & & $7.52 E-10$ \\
\hline 39 & Pa-233 & & 2.77E-05 \\
\hline 40 & Th-234 & & 5.46E-06 \\
\hline 41 & Pa-234 & & 1.31E-05 \\
\hline 42 & Np-239 & & 4.82E-05 \\
\hline 43 & Th-231 & & $4.01 E-09$ \\
\hline$\overline{44}$ & Ra-228 & & $2.56 E-14$ \\
\hline 45 & $A C-228$ & & $4.98 E-08$ \\
\hline 46 & & & \\
\hline 47 & Total & $1.05 E+02$ & $3.23 E+01$ \\
\hline
\end{tabular}




\begin{tabular}{|c|c|c|c|}
\hline & $\mathrm{B}$ & $\mathrm{H}$ & I \\
\hline 9 & Nuclide & CEDE (mrem per $\left.u C l / m^{\wedge} 2\right)$ & External EDE (mrem per uCi/m^2) \\
\hline 10 & Am-241 & $=\$ C \$ 7^{*} \$ C \$ 3^{*} \$ C \$ 4^{*} \$ C \$ 5^{*} \$ C \$ 6^{*}$ F $10^{*}$ Relocation!H10*O10 & $=\$ C \$ 7^{*} \$ D 10^{*}$ Relocation!E10*\$C\$35/\$E $\$ 35^{*} 010$ \\
\hline 11 & Am-243 & $=\$ C \$ 7^{*} \$ C \$ 3^{*} \$ C \$ 4^{*} \$ C \$ 5^{*} \$ C \$ 6^{*}$ F11*Relocation!H11*011 & $=\$ C \$ 7^{*} \$ D 11^{*}$ Relocation!E11*\$C $\$ 35 / \$ E \$ 35^{*} 011$ \\
\hline 12 & Ba-133 & $=\$ C \$ 7^{*} \$ C \$ 3^{*} \$ C \$ 4^{*} \$ C \$ 5^{*} \$ C \$ 6^{*} F 12^{*}$ Relocation!H12*O12 & $=\$ C \$ 7^{*} \$ D 12^{\star}$ Relocation!E12*\$C\$35/\$E $\$ 35^{\star} \mathrm{O} 12$ \\
\hline 13 & Ce-144 & $=\$ C \$ 7^{*} \$ C \$ 3^{*} \$ C \$ 4^{*} \$ C \$ 5^{*} \$ C \$ 6^{*} F 13^{*}$ Relocation!H13*013 & $=\$ C \$ 7^{*} \$ D 13^{*}$ Relocation!E13*\$C $\$ 35 / \$ E \$ 35^{*} 013$ \\
\hline 14 & CF-249 & $=\$ C \$ 7^{*} \$ C \$ 3^{*} \$ C \$ 4^{*} \$ C \$ 5^{*} \$ C \$ 6^{*} F 14^{*}$ Relocation!H14*O14 & $=\$ C \$ 7^{*} \$ D 14^{*}$ Relocation!E14*\$C\$35/\$E $\$ 35^{*} 014$ \\
\hline 15 & Cf-252 & $=\$ C \$ 7^{*} \$ C \$ 3^{*} \$ C \$ 4^{*} \$ C \$ 5^{*} \$ C \$ 6^{*} F 15^{*}$ Relocation!H15*015 & $=\$ C \$ 7^{*} \$ D 15^{*}$ Relocation!E15*\$C\$35/\$E\$35*015 \\
\hline 16 & $\mathrm{Cm}-244$ & $=\$ C \$ 7^{*} \$ C \$ 3^{*} \$ C \$ 4^{*} \$ C \$ 5^{*} \$ C \$ 6^{*} F 16^{*}$ Relocation!H16*016 & $=\$ C \$ 7^{*} \$ D 16^{*}$ Relocation!E16*\$C $\$ 35 / \$ E \$ 35^{\star} 016$ \\
\hline 17 & $\mathrm{Cm}-246$ & $=\$ C \$ 7^{*} \$ C \$ 3^{*} \$ C \$ 4^{*} \$ C \$ 5^{*} \$ C \$ 6^{*} F 17^{*}$ Relocation!H17*017 & $=\$ C \$ 7^{*} \$ D 17^{*}$ Relocation!E17*\$C\$35/\$E\$35*017 \\
\hline 18 & Co-60 & $=\$ C \$ 7^{*} \$ C \$ 3^{*} \$ C \$ 4^{*} \$ C \$ 5^{*} \$ C \$ 6^{*} F 18^{*}$ Relocation!H18*018 & $=\$ C \$ 7^{*} \$ D 18^{*}$ Relocation!E18*\$C\$35/\$E $\$ 35^{*} 018$ \\
\hline 19 & Cs-137 & $=\$ C \$ 7^{*} \$ C \$ 3^{*} \$ C \$ 4^{*} \$ C \$ 5^{*} \$ C \$ 6^{*} F 19^{*}$ Relocation!H19*019 & $=\$ C \$ 7^{*} \$ D 19^{*}$ Relocation!E19*\$C\$35/\$E $\$ 35^{*} 019$ \\
\hline 20 & H-3 & & \\
\hline 21 & Np-237 & $=\$ C \$ 7^{*} \$ C \$ 3^{*} \$ C \$ 4^{*} \$ C \$ 5^{*} \$ C \$ 6^{*} F 21^{*}$ Relocation! $H 21^{*} \mathrm{O} 21$ & $=\$ C \$ 7^{*} \$ D 21^{*}$ Relocation!E21*\$C $\$ 35 / \$ E \$ 35^{*} \mathrm{O} 21$ \\
\hline 22 & Pm-147 & $=\$ C \$ 7^{*} \$ C \$ 3^{*} \$ C \$ 4^{*} \$ C \$ 5^{*} \$ C \$ 6^{*} F 22^{*}$ Relocation!H22*O22 & $=\$ C \$ 7^{*} \$ D 22^{*}$ Relocation!E22*\$C $\$ 35 / \$ E \$ 35^{*} 022$ \\
\hline 23 & Pu-238 & $=\$ C \$ 7^{*} \$ C \$ 3^{*} \$ C \$ 4^{*} \$ C \$ 5^{*} \$ C \$ 6^{*} F 23^{*}$ Relocation $1 \mathrm{H} 23^{*} \mathrm{O} 23$ & $=\$ C \$ 7^{*} \$ D 23^{*}$ Relocation!E23*\$C $\$ 35 / \$ E \$ 35^{*} 023$ \\
\hline 24 & Pu-239 & $=\$ C \$ 7^{*} \$ C \$ 3^{*} \$ C \$ 4^{*} \$ C \$ 5^{*} \$ C \$ 6^{*} \mathrm{~F} 24^{*}$ Relocation!H24*O24 & $=\$ C \$ 7^{*} \$ D 24^{*}$ Relocation!E24*\$C\$35/\$E\$35*O24 \\
\hline 25 & Pu mix-239/241 & & \\
\hline 26 & Pu-240 & 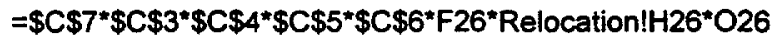 & $=\$ C \$ 7^{*} \$ D 26^{*}$ Relocation!E26*\$C $\$ 35 / \$ E \$ 35^{*} \mathrm{O} 26$ \\
\hline 27 & Pu-241 & $=\$ C \$ 7^{*} \$ C \$ 3^{*} \$ C \$ 4^{*} \$ C \$ 5^{*} \$ C \$ 6^{*} F 27^{*}$ Relocation!H27*027 & $=\$ C \$ 7^{\star} \$ D 27^{*}$ Relocation!E27*\$C\$35/\$E\$35*O27 \\
\hline 28 & Pu pure-242 & $=\$ C \$ 7^{*} \$ C \$ 3^{*} \$ C \$ 4^{*} \$ C \$ 5^{*} \$ C \$ 6^{*} F 28^{*}$ Relocation!H28*O28 & $=\$ C \$ 7^{*} \$ D 28^{*}$ Relocation!E28*\$C $\$ 35 / \$ E \$ 35^{*} \mathrm{O} 28$ \\
\hline 29 & Sr-90 & $=\$ C \$ 7^{*} \$ C \$ 3^{*} \$ C \$ 4^{*} \$ C \$ 5^{*} \$ C \$ 6^{*}$ F29*Relocation!H29*O29 & $=\$ C \$ 7^{*} \$ D 29^{*}$ Relocation!E29*\$C $\$ 35 / \$ E \$ 35^{*} \mathrm{O} 29$ \\
\hline 30 & TC-99 & $=\$ C \$ 7^{*} \$ C \$ 3^{*} \$ C \$ 4^{*} \$ C \$ 5^{*} \$ C \$ 6^{*} \mathrm{~F} 30^{*}$ Relocation!H30*O30 & $=\$ C \$ 7^{*} \$ D 30^{*}$ Relocation!E30*\$C $\$ 35 / \$ E \$ 35^{*} 030$ \\
\hline 31 & Th-232 & $=\$ C \$ 7^{*} \$ C \$ 3^{*} \$ C \$ 4^{*} \$ C \$ 5^{*} \$ C \$ 6^{*} F 31^{*}$ Relocation!H31*O31 & $=\$ C \$ 7^{*} \$ D 31^{*}$ Relocation!E31*\$C $\$ 35 / \$ E \$ 35^{*} \mathrm{O} 31$ \\
\hline 32 & TI-204 & $=\$ C \$ 7^{*} \$ C \$ 3^{*} \$ C \$ 4^{*} \$ C \$ 5^{*} \$ C \$ 6^{*} F 32^{*}$ Relocation!H32*O32 & $=\$ C \$ 7^{*} \$ D 32^{*}$ Relocation!E32*\$C\$35/\$E\$35*O32 \\
\hline 33 & U-235 & $=\$ C \$ 7^{*} \$ C \$ 3^{*} \$ C \$ 4^{*} \$ C \$ 5^{*} \$ C \$ 6^{*} F 33^{*}$ Relocation!H33*O33 & $=\$ C \$ 7^{*} \$ D 33^{*}$ Relocation!E33*\$C\$35/\$E $\$ 35^{*} 033$ \\
\hline 34 & U-238 & $=\$ C \$ 7^{*} \$ C \$ 3^{*} \$ C \$ 4^{*} \$ C \$ 5^{*} \$ C \$ 6^{*} \mathrm{~F} 34^{*}$ Relocation!H34*O34 & $=\$ C \$ 7^{*} \$ D 34^{*}$ Relocation!E34*\$C $\$ 35 / \$ E \$ 35^{*} 034$ \\
\hline 35 & Total & & \\
\hline 36 & $\mathrm{Ba}-137 \mathrm{~m}$ & & $=\$ C \$ 7^{*} \$ D 36^{*}$ Relocation!E36*\$C $\$ 35 / \$ E \$ 35^{*} 036$ \\
\hline 37 & $Y-90$ & & $=\$ C \$ 7^{*} \$ D 37^{*}$ Relocation!E37*\$C\$35/\$E\$35*037 \\
\hline 38 & Pr-144 & & $=\$ C \$ 7^{*} \$ D 38^{*}$ Relocation!E38*\$C $\$ 35 / \$ E \$ 35^{*} 038$ \\
\hline 39 & $\mathrm{~Pa}-233$ & & $=\$ C \$ 7^{*} \$ D 39^{*}$ Relocation!E39*\$C $\$ 35 / \$ E \$ 35^{*} 039$ \\
\hline 40 & Th-234 & & $=\$ C \$ 7^{*} \$ D 40^{*}$ Relocation!E $40^{*} \$ C \$ 35 / \$ E \$ 35^{*} 040$ \\
\hline 41 & Pa-234 & & $=\$ C \$ 7^{*} \$ D 41^{*}$ Relocation!E41*\$C $\$ 35 / \$ E \$ 35^{*} 041$ \\
\hline 42 & Np-239 & & $=\$ C \$ 7^{*} \$ D 42^{*}$ Relocation!E42*\$C $\$ 35 / \$ E \$ 35^{*} 042$ \\
\hline 43 & Th-231 & & $=\$ C \$ 7^{*} \$ D 43^{*}$ Relocation!E43*\$C $\$ 35 / \$ E \$ 35^{*} 043$ \\
\hline 44 & Ra-228 & & $=\$ C \$ 7^{*} \$ D 44^{*}$ Relocation!E $44^{*} \$ C \$ 35 / \$ E \$ 35^{*} O 44$ \\
\hline 45 & Ac-228 & & $=\$ C \$ 7^{*} \$ D 45^{*}$ Relocation!E $45^{\star} \$ C \$ 35 / \$ E \$ 35^{*}$ O45 \\
\hline 46 & & & \\
\hline 47 & Total & $=$ SUM $(H 10: H 46)$ & $=\operatorname{SUM}(110: 146)$ \\
\hline
\end{tabular}




\begin{tabular}{|c|c|c|c|}
\hline & $\bar{B}$ & $\bar{K}$ & L \\
\hline 9 & Nuclide & $\begin{array}{c}\text { Skin Dose (mrem per } \\
\left.u C i / m^{\wedge} 2\right)\end{array}$ & BS Dose (mrem per uCl//m^2) \\
\hline 10 & Am-241 & 5.77E-02 & $3.50 E+02$ \\
\hline 11 & Am-243 & 2.15E-03 & $1.07 E+01$ \\
\hline 12 & $B a-133$ & $6.36 E-12$ & 4.04E-14 \\
\hline 13 & $\mathrm{Ce}-144$ & $7.83 E-06$ & 5.02E-07 \\
\hline 14 & Cf-249 & 1.27E-04 & 2.97E-01 \\
\hline 15 & $C f-252$ & $1.38 E-03$ & $1.58 E+01$ \\
\hline 16 & $\mathrm{Cm}-244$ & 2.74E-03 & $2.13 E+02$ \\
\hline 17 & $\mathrm{Cm}-246$ & $1.22 \mathrm{E}-04$ & $1.20 E+01$ \\
\hline 18 & Co-60 & $4.33 E+01$ & 7.45E-02 \\
\hline 19 & Cs-137 & $6.92 E+00$ & 7.60E-02 \\
\hline 20 & $\mathrm{H}-3$ & & \\
\hline 21 & Np-237 & $6.63 E-05$ & 8.67E-01 \\
\hline 22 & Pm-147 & 2.33E-12 & 3.67E-09 \\
\hline 23 & Pu-238 & 8.28E-03 & $3.72 E+02$ \\
\hline 24 & Pu-239 & $5.42 E-03$ & $5.37 E+02$ \\
\hline 25 & Pu mix-239/241 & & \\
\hline 26 & Pu-240 & $3.03 E-03$ & $1.22 E+02$ \\
\hline 27 & Pu-241 & $0.00 E+\infty$ & $1.19 E+02$ \\
\hline 28 & Pu pure-242 & 1.74E-05 & 7.56E-01 \\
\hline 29 & Sr-90 & $4.22 E+00$ & $6.72 E-01$ \\
\hline 30 & Tc-99 & $5.83 E-18$ & 1.17E-16 \\
\hline 31 & Th-232 & 1.96E-07 & 1.73E-01 \\
\hline 32 & TI-204 & 2.35E-08 & $2.80 E-12$ \\
\hline 33 & $U-235$ & $8.48 E-06$ & 7.83E-06 \\
\hline 34 & U-238 & 2.39E-05 & $5.60 \mathrm{E}-04$ \\
\hline 35 & Total & & \\
\hline 36 & Ba-137m & 4.46E-04 & \\
\hline 37 & $Y-90$ & $3.55 E+00$ & \\
\hline 38 & Pr-144 & $3.76 E-07$ & \\
\hline 39 & Pa-233 & 3.85E-05 & \\
\hline 40 & Th-234 & 9.57E-06 & \\
\hline 41 & Pa-234 & 2.69E-05 & \\
\hline 42 & Np-239 & 7.49E-05 & \\
\hline 43 & Th-231. & 1.35E-08 & \\
\hline 44 & Ra-228 & $3.08 E-13$ & \\
\hline 45 & $A C-228$ & 2.76E-07 & \\
\hline 46 & & & \\
\hline 47 & Total & $5.81 E+01$ & $1.75 E+03$ \\
\hline
\end{tabular}




\begin{tabular}{|c|c|c|c|}
\hline & $\mathbf{B}$ & $\mathrm{K}$ & $L$ \\
\hline 9 & Nuclide & Skin Dose (mrem per uCi/m^2) & BS Dose (mrem per uCl/m^2) \\
\hline 10 & Am-241 & $=\$ C \$ 7^{*} \$ D 10^{*}$ Relocation $\mid \$ F 10^{*} \$ C \$ 35 / \$ E \$ 35^{*} 010$ & $=\$ C \$ 7^{*} \$ C \$ 3^{*} \$ C \$ 4^{*} \$ C \$ 5^{*} \$ C \$ 6^{*} \$ F 10^{*}$ Relocation! $\$ 10^{*} 01 \phi$ \\
\hline 11 & Am-243 & $=\$ C \$ 7^{*} \$ D 11^{*}$ Relocation! $\$ F 11^{*} \$ C \$ 35 / \$ E \$ 35^{*} 011$ & $=\$ C \$ 7^{*} \$ C \$ 3^{*} \$ C \$ 4^{*} \$ C \$ 5^{*} \$ C \$ 6^{*} \$ F 11^{*}$ Relocation! $\$ 11^{*} O 1$ \\
\hline 12 & $\mathrm{Ba}-133$ & $=\$ C \$ 7 * \$ D 12 *$ Relocation!\$F12*\$C $\$ 35 / \$ E \$ 35^{*} 012$ & $=\$ C \$ 7^{*} \$ C \$ 3^{*} \$ C \$ 4^{*} \$ C \$ 5^{*} \$ C \$ 6^{*} \$ F 12^{*}$ Relocation! $\$ 112^{*} 012$ \\
\hline 13 & $\mathrm{Ce}-144$ & $=\$ C \$ 7^{*} \$ D 13^{*}$ Relocation!\$F13*\$C\$35/\$E\$35*013 & $=\$ C \$ 7^{*} \$ C \$ 3^{*} \$ C \$ 4^{*} \$ C \$ 5^{*} \$ C \$ 6^{*} \$ F 13^{*}$ Relocation! $\left.\$ 13^{*} 01\right\}$ \\
\hline 14 & $\mathrm{Ct}-249$ & $=\$ C \$ 7^{*} \$ D 14^{*}$ Relocation!\$F14*\$C $35 / \$ E \$ 35^{*} 014$ & $=\$ C \$ 7^{*} \$ C \$ 3^{*} \$ C \$ 4^{*} \$ C \$ 5^{*} \$ C \$ 6^{*} \$ F 14^{*}$ Relocation $\mid \$ J 14^{*} 01$ \\
\hline 15 & Cf-252 & $=\$ C \$ 7^{*} \$ D 15^{*}$ Relocation!\$F15*\$C $\$ 35 / \$ E \$ 35^{*} \mathrm{O} 15$ & $=\$ C \$ 7^{*} \$ C \$ 3^{*} \$ C \$ 4^{*} \$ C \$ 5^{*} \$ C \$ 6^{*} \$ F 15^{*}$ Relocation $\mid \$ 15^{*} 01 \$$ \\
\hline 16 & $\mathrm{Cm}-244$ & $=\$ C \$ 7^{*} \$ D 16^{*}$ Relocation $\mid \$ F 16^{*} \$ C \$ 35 / \$ E \$ 35^{\star} \mathrm{O} 16$ & $=\$ C \$ 7^{*} \$ C \$ 3^{*} \$ C \$ 4^{*} \$ C \$ 5^{*} \$ C \$ 6^{*} \$ F 16^{*}$ Relocation $1 \$ 16^{*} 01$ \\
\hline 17 & $\mathrm{Cm}-246$ & $=\$ C \$ 7^{*} \$ D 17^{*}$ Relocation!\$F17*\$C\$35/\$E\$35*017 & $=\$ C \$ 7^{\star} \$ C \$ 3^{\star} \$ C \$ 4^{\star} \$ C \$ 5^{\star} \$ C \$ 6^{*} \$ F 17^{\star}$ Relocation! $\$ J 17^{*} 017$ \\
\hline 18 & Co-60 & $=\$ C \$ 7^{*} \$ D 18^{*}$ Relocation!\$F $18^{*} \$ C \$ 35 / \$ E \$ 35^{*} 018$ & $=\$ C \$ 7^{*} \$ C \$ 3^{*} \$ C \$ 4^{*} \$ C \$ 5^{*} \$ C \$ 6^{*} \$ F 18^{*}$ Relocation! $\$ J 18^{*} 01 \beta$ \\
\hline 19 & Cs-137 & $=\$ C \$ 7^{\star} \$ D 19^{*}$ Relocation!\$F19*\$C\$35/\$E $\$ 35^{\star} 019$ & $=\$ C \$ 7^{*} \$ C \$ 3^{*} \$ C \$ 4^{*} \$ C \$ 5^{*} \$ C \$ 6^{*} \$ F 19^{*}$ Relocation $\$ \mathrm{~J} 19^{*} 01$ \\
\hline 20 & $\mathrm{H}-3$ & & \\
\hline 21 & Np-237 & $=\$ C \$ 7^{\star} \$ D 21 *$ Relocation!\$F21*\$C\$35/\$E \$35*O21 & $=\$ C \$ 7^{*} \$ C \$ 3^{*} \$ C \$ 4^{*} \$ C \$ 5^{*} \$ C \$ 6^{*} \$ F 21^{*}$ Relocation $1 \$ 21^{*} \mathrm{O} 2$ \\
\hline 22 & Pm-147 & $=\$ C \$ 7^{\star} \$ D 22^{*}$ Relocation!\$F22*\$C $\$ 35 / \$ E \$ 35^{\star} \mathrm{O} 22$ & $=\$ C \$ 7^{\star} \$ C \$ 3^{\star} \$ C \$ 4^{\star} \$ C \$ 5^{*} \$ C \$ 6^{*} \$ F 22^{*}$ Relocation! $\$ 22^{*} \mathrm{O} 2$ \\
\hline 23 & $\mathrm{Pu}-238$ & $=\$ C \$ 7^{*} \$ D 23^{*}$ Relocation!\$F23*\$C $\$ 35 / \$ E \$ 35^{*} \mathrm{O} 23$ & $=\$ C \$ 7^{*} \$ C \$ 3^{*} \$ C \$ 4^{*} \$ C \$ 5^{*} \$ C \$ 6^{*} \$ F 23^{*}$ Relocation $\$$ J $23^{*} \mathrm{O} 2$ \\
\hline 24 & Pu-239 & $=\$ C \$ 7^{*} \$ D 24^{\star}$ Relocation!\$F24*\$C $\$ 35 / \$ E \$ 35^{\star} \mathrm{O} 24$ & $=\$ C \$ 7^{*} \$ C \$ 3^{*} \$ C \$ 4^{*} \$ C \$ 5^{*} \$ C \$ 6^{*} \$ F 24^{*}$ Relocation! $\$ 24^{*} \mathrm{O} 2$ \\
\hline 25 & Pu mix-239/241 & & \\
\hline 26 & Pu-240 & $=\$ C \$ 7^{*} \$ D 26^{*}$ Relocation!\$F26*\$C $\$ 35 / \$ E \$ 35^{*}$ O26 & $=\$ C \$ 7^{*} \$ C \$ 3^{*} \$ C \$ 4^{*} \$ C \$ 5^{*} \$ C \$ 6^{*} \$ F 26^{*}$ Relocation $1 \$ 26^{*} \mathrm{O} 2$ \\
\hline 27 & Pu-241 & $=\$ C \$ 7^{\star} \$ D 27^{\star}$ Relocation!\$F27*\$C $\$ 35 / \$ E \$ 35^{\star} \mathrm{O} 27$ & $=\$ C \$ 7^{\star} \$ C \$ 3^{\star} \$ C \$ 4^{*} \$ C \$ 5^{*} \$ C \$ 6^{*} \$ F 27^{\star}$ Relocation $1 \$ 27^{\star} \mathrm{O} 2$ \\
\hline 28 & Pu pure-242 & $=\$ C \$ 7^{\star} \$ D 28^{*}$ Relocation!\$F28*\$C\$35/\$E\$35*O28 & $=\$ C \$ 7^{*} \$ C \$ 3^{*} \$ C \$ 4^{*} \$ C \$ 5^{*} \$ C \$ 6^{*} \$ F 28^{*}$ Relocation! $\$ 28^{*} O 2$ \\
\hline 29 & Sr-90 & $=\$ C \$ 7 * \$ D 29 *$ Relocation!\$F29*\$C \$35/\$E \$35*O29 & $=\$ C \$ 7^{*} \$ C \$ 3^{*} \$ C \$ 4^{*} \$ C \$ 5^{*} \$ C \$ 6^{*} \$ F 29^{*}$ Relocation $1 \$ \mathrm{~J} 29^{*} 02$ \\
\hline 30 & Tc-99 & $=\$ C \$ 7^{\star} \$ D 30^{*}$ Relocation!\$F30*\$C\$35/\$E $\$ 35^{*} \mathrm{O} 30$ & $=\$ C \$ 7^{*} \$ C \$ 3^{*} \$ C \$ 4^{*} \$ C \$ 5^{*} \$ C \$ 6^{*} \$ F 30^{*}$ Relocation! $\$ J 30^{*} 03$ \\
\hline 31 & Th-232 & $=\$ C \$ 7 * \$ D 31 *$ Relocation!\$F31*\$C\$35/\$E $\$ 35^{*} \mathrm{O} 31$ & $=\$ C \$ 7^{*} \$ C \$ 3^{*} \$ C \$ 4^{*} \$ C \$ 5^{*} \$ C \$ 6^{*} \$ F 31^{*}$ Relocation $\mid \$ J 31^{*} O 3$ \\
\hline 32 & TI-204 & $=\$ C \$ 7^{\star} \$ D 32^{*}$ Relocation!\$F32*\$C\$35/\$E $\$ 35^{\star} \mathrm{O} 32$ & $=\$ C \$ 7^{*} \$ C \$ 3^{*} \$ C \$ 4^{*} \$ C \$ 5^{*} \$ C \$ 6^{*} \$ F 32^{*}$ Relocation! $\$ 32^{*} 03$ \\
\hline 33 & U-235 & $=\$ C \$ 7^{*} \$ D 33^{*}$ Relocation!\$F33*\$C\$35/\$E\$35*O33 & $=\$ C \$ 7^{*} \$ C \$ 3^{*} \$ C \$ 4^{*} \$ C \$ 5^{*} \$ C \$ 6^{*} \$ F 33^{*}$ Relocation $\mid \$ J 33^{*} O 3$ \\
\hline 34 & $\mathrm{U}-238$ & $=\$ C \$ 7^{*} \$ D 34 *$ Relocation!\$F34*\$C\$35/\$E \$35*O34 & $=\$ C \$ 7^{*} \$ C \$ 3^{*} \$ C \$ 4^{*} \$ C \$ 5^{*} \$ C \$ 6^{*} \$ F 34^{*}$ Relocation! $\$ 34^{*} \mathrm{O} 3$ \\
\hline$\overline{35}$ & Total & & \\
\hline 36 & $\mathrm{Ba}-137 \mathrm{~m}$ & $=\$ C \$ 7^{*} \$ D 36^{*}$ Relocation!\$F36*\$C\$35/\$E $\$ 35^{*} 036$ & \\
\hline 37 & $Y-90$ & $=\$ C \$ 7^{\star} \$ D 37^{*}$ Relocation!\$F37*\$C\$35/\$E \$35*037 & \\
\hline 38 & $\operatorname{Pr}-144$ & $=\$ C \$ 7^{*} \$ D 38^{*}$ Relocation!\$F38*\$C\$35/\$E $\$ 35^{*} 038$ & \\
\hline 39 & Pa-233 & $=\$ C \$ 7^{*} \$ D 39 *$ Relocation!\$F39*\$C\$35/\$E\$35*039 & \\
\hline 40 & Th-234 & $=\$ C \$ 7 * \$ D 40^{*}$ Relocation!\$F40*\$C $\$ 35 / \$ E \$ 35^{*} 040$ & \\
\hline 41 & $\mathrm{~Pa}-234$ & $=\$ C \$ 7^{*} \$ D 41^{*}$ Relocation!\$F41*\$C\$35/\$E\$35*041 & $\cdot$ \\
\hline 42 & $N p-239$ & $=\$ C \$ 7^{*} \$ D 42^{*}$ Relocation!\$F $42^{*} \$ C \$ 35 / \$ E \$ 35^{*} 042$ & \\
\hline 43 & Th-231 & $=\$ C \$ 7^{*} \$ D 43^{*}$ Relocation!\$F $43^{*} \$ C \$ 35 / \$ E \$ 35^{*} \mathrm{O} 43$ & \\
\hline$\overline{44}$ & Ra-228 & $=\$ C \$ 7^{*} \$ D 44^{*}$ Relocation!\$F $44^{*} \$ C \$ 35 / \$ E \$ 35^{\star}$ O44 & \\
\hline 45 & Ac-228 & $=\$ C \$ 7^{*} \$ 045^{*}$ Relocation!\$F45*\$C \$35/\$E\$35*045 & \\
\hline 46 & & & \\
\hline 47 & Total & $=S U M(K 10: K 46)$ & $=\operatorname{SUM}(\mathrm{L} 10: \mathrm{L} 46)$ \\
\hline
\end{tabular}




\begin{tabular}{|c|c|c|c|}
\hline & $\bar{B}$ & $\bar{M}$ & $\overline{\mathbf{N}}$ \\
\hline 9 & Nuclide & Halflife (years) & Lambda1 \\
\hline 10 & Am-241 & $4,580 E+02$ & $1.51 E-03$ \\
\hline 11 & $A m-243$ & $7.950 E+03$ & 8.72E-05 \\
\hline 12 & $\mathrm{Ba}-133$ & $1.050 E+01$ & 6.60E-02 \\
\hline 13 & $\mathrm{Ce}-144$ & $7.781 E-01$ & 8.91E-01 \\
\hline 14 & Cf-249 & $3.600 E+02$ & $1.93 E-03$ \\
\hline 15 & Cf-252 & $2.646 \mathrm{E}+00$ & 2.62E-01 \\
\hline 16 & $\mathrm{Cm}-244$ & $1.760 E+01$ & 3.94E-02 \\
\hline 17 & $\mathrm{Cm}-246$ & $5.500 E+03$ & $1.26 E-04$ \\
\hline 18 & Co-60 & $5.260 E+00$ & 1.32E-01 \\
\hline 19 & Cs-137 & $3.000 E+01$ & 2.31E-02 \\
\hline 20 & $\mathrm{H}-3$ & $1.230 E+01$ & 5.63E-02 \\
\hline 21 & $\mathrm{~Np}-237$ & $2.140 E+06$ & 3.24E-07 \\
\hline 22 & Pm-147 & $2.620 E+00$ & 2.65E-01 \\
\hline 23 & Pu-238 & $8.640 E+01$ & $8.02 E-03$ \\
\hline 24 & Pu-239 & $2.439 E+04$ & 2.84E-05 \\
\hline 25 & Pu mix-239/241 & & \\
\hline 26 & Pu-240 & $6.580 E+03$ & 1.05E-04 \\
\hline 27 & Pu-241 & $1.320 E+01$ & $5.25 E-02$ \\
\hline 28 & Pu pure-242 & $3.790 E+05$ & 1.83E-06 \\
\hline 29 & Sr-90 & $2.810 E+01$ & 2.47E-02 \\
\hline 30 & Tc-99 & 2.120E+05 & 3.27E-06 \\
\hline 31 & Th-232 & $1.400 E+10$ & 4.95E-11 \\
\hline 32 & TI-204 & $3.800 E+00$ & $1.82 E-01$ \\
\hline 33 & U-235 & $7.100 E+08$ & $9.76 E-10$ \\
\hline 34 & U-238 & $4.510 E+09$ & $1.54 \mathrm{E}-10$ \\
\hline 35 & Total & & \\
\hline 36 & $\mathrm{Ba}-137 \mathrm{~m}$ & 4.855E-06 & $1.43 E+05$ \\
\hline 37 & $Y-90$ & 7.317E-03 & $9.47 E+01$ \\
\hline 38 & Pr-144 & $3.288 E-05$ & 2.11E+04 \\
\hline 39 & Pa-233 & 7.397E-02 & $9.37 E+00$ \\
\hline 40 & Th-234 & $6.603 E-02$ & $1.05 E+01$ \\
\hline 41 & $\mathrm{~Pa}-234$ & 7.648E-04 & $9.06 E+02$ \\
\hline 42 & Np-239 & $6.452 E-03$ & 1.07E+02 \\
\hline 43 & Th-231 & 2.913E-03 & $2.38 E+02$ \\
\hline 44 & $R a-228$ & $5.750 E+00$ & $1.21 E-01$ \\
\hline 45 & Ac-228 & $6.998 \mathrm{E}-04$ & $9.90 E+02$ \\
\hline
\end{tabular}




\begin{tabular}{|c|c|c|c|}
\hline & $\bar{B}$ & $M$ & $\mathbf{N}$ \\
\hline 9 & Nuclide & Halflife (years) & Lambda1 \\
\hline 10 & Am-241 & $=T_{\text {_halfic } 2}$ & $=0.693 / \mathrm{M} 10$ \\
\hline 11 & Am-243 & $=T$ halfic3 & $=0.693 / \mathrm{M} 11$ \\
\hline 12 & Ba-133 & $=T_{-}$half $1 \mathrm{C}_{4}$ & $=0.693 / \mathrm{M} 12$ \\
\hline 13 & Ce-144 & $=T$ half!C5 & $=0.693 / \mathrm{M} 13$ \\
\hline 14 & Cf-249 & $=T$ half!C6 & $=0.693 / \mathrm{M} 14$ \\
\hline 15 & Cf-252 & $=T$ _half!C7 & $=0.693 / \mathrm{M} 15$ \\
\hline 16 & $\mathrm{Cm}-244$ & $=T_{-}$half!C8 & $=0.693 / \mathrm{M} 16$ \\
\hline 17 & $\mathrm{Cm}-246$ & $=T_{-}$half!C9 & $=0.693 / \mathrm{M} 17$ \\
\hline 18 & Co-60 & $=T$ _half!C10 & $=0.693 / \mathrm{M} 18$ \\
\hline 19 & Cs-137 & $=T$ half!C11 & $=0.693 / \mathrm{M} 19$ \\
\hline 20 & $\mathrm{H}-3$ & $=T$ _half!C12 & $=0.693 / \mathrm{M} 20$ \\
\hline 21 & $\mathrm{~Np}-237$ & $=T$ halflC13 & $=0.693 / \mathrm{M} 21$ \\
\hline 22 & Pm-147 & $=T_{\text {_halflC14 }}$ & $=0.693 / \mathrm{M} 22$ \\
\hline 23 & Pu-238 & $=T$ _halflC15 & $=0.693 / \mathrm{M} 23$ \\
\hline 24 & Pu-239 & $=T$ _half!C16 & $=0.693 / \mathrm{M} 24$ \\
\hline 25 & Pu mix-239/241 & & \\
\hline 26 & Pu-240 & $=T$ _half!C18 & $=0.693 / \mathrm{M} 26$ \\
\hline 27 & Pu-241 & $=T_{\text {_half }}$ C 19 & $=0.693 / \mathrm{M} 27$ \\
\hline 28 & Pu pure-242 & $=T_{\text {_halfic }} 20$ & $=0.693 / \mathrm{M} 28$ \\
\hline 29 & Sr-90 & $=T$ _half!C21 & $=0.693 / \mathrm{M} 29$ \\
\hline 30 & Tc-99 & $=T$ half!C22 & $=0.693 / \mathrm{M} 30$ \\
\hline 31 & Th-232 & $=T$ half $! C 23$ & $=0.693 / \mathrm{M} 31$ \\
\hline 32 & T1-204 & $=T_{\text {_half }} \mathrm{C} 24$ & $=0.693 / \mathrm{M} 32$ \\
\hline 33 & U-235 & $=T$ T_half!C25 & $=0.693 / \mathrm{M} 33$ \\
\hline 34 & $\mathrm{U}-238$ & $=T$ half!C26 & $=0.693 / \mathrm{M} 34$ \\
\hline 35 & Total & & \\
\hline 36 & Ba-137m & $=T$ half!C28 & $=0.693 / \mathrm{M} 36$ \\
\hline 37 & $Y-90$ & $=T$ _half!C29 & $=0.693 / \mathrm{M} 37$ \\
\hline 38 & Pr-144 & $=T$ _half!C30 & $=0.693 / \mathrm{M} 38$ \\
\hline 39 & $\mathrm{~Pa}-233$ & $=$ T_half!C31 & $=0.693 / \mathrm{M} 39$ \\
\hline 40 & Th-234 & $=T^{-}$half!C32 & $=0.693 / \mathrm{M} 40$ \\
\hline 41 & Pa-234 & $=T_{-}$half $\mathrm{C} 33$ & $=0.693 / \mathrm{M} 41$ \\
\hline 42 & $\mathrm{~Np}-239$ & $=T$ _half!C34 & $=0.693 / \mathrm{M} 42$ \\
\hline 43 & Th-231 & $=T^{-}$half!C35 & $=0.693 / \mathrm{M} 43$ \\
\hline 44 & Ra-228 & $=T$-half!C36 & $=0.693 / \mathrm{M} 44$ \\
\hline 45 & Ac-228 & $=T$ half!C37 & $=0.693 / \mathrm{M} 45$ \\
\hline
\end{tabular}




\begin{tabular}{|c|c|c|}
\hline & $\bar{B}$ & 0 \\
\hline 9 & Nuclide & Weathering Factor (1 year) \\
\hline 10 & Am-241 & $7.455 E-01$ \\
\hline 11 & Am-243 & $7.460 \mathrm{E}-01$ \\
\hline 12 & $\mathrm{Ba}-133$ & $7.242 E-01$ \\
\hline 13 & $\mathrm{Ce}-144$ & 5.146E-01 \\
\hline 14 & $C f-249$ & $7.454 E-01$ \\
\hline 15 & Ct-252 & $6.644 E-01$ \\
\hline 16 & $\mathrm{Cm}-244$ & $7.329 E-01$ \\
\hline 17 & $\mathrm{Cm}-246$ & $7.460 E-01$ \\
\hline 18 & Co-60 & 7.033E-01 \\
\hline 19 & Cs-137 & 7.383E-01 \\
\hline 20 & $\mathrm{H}-3$ & 7.273E-01 \\
\hline 21 & Np-237 & $7.460 E-01$ \\
\hline 22 & $P m-147$ & 6.637E-01 \\
\hline 23 & Pu-238 & 7.433E-01 \\
\hline 24 & Pu-239 & $7.460 E-01$ \\
\hline 25 & Pu mix-239/241 & \\
\hline 26 & Pu-240 & $7.460 E-01$ \\
\hline 27 & $P u-241$ & $7.286 E-01$ \\
\hline 28 & Pu pure-242 & $7.460 E-01$ \\
\hline 29 & Sr-90 & $7.378 E-01$ \\
\hline 30 & Tc-99 & $7.460 E-01$ \\
\hline 31 & Th-232 & $7.460 \mathrm{E}-01$ \\
\hline 32 & TI-204 & 6.878E-01 \\
\hline 33 & U-235 & $7.460 \mathrm{E}-01$ \\
\hline 34 & $U-238$ & 7.460 E-01 \\
\hline 35 & Total & \\
\hline 36 & $\mathrm{Ba}-137 \mathrm{~m}$ & $7.006 \mathrm{E}-06$ \\
\hline 37 & $Y-90$ & $1.048 E-02$ \\
\hline 38 & Pr-144 & 4.744E-05 \\
\hline 39 & Pa-233 & 9.947E-02 \\
\hline 40 & Th-234 & $8.942 E-02$ \\
\hline 41 & $\mathrm{~Pa}-234$ & 1.103E-03 \\
\hline 42 & Np-239 & 9.249E-03 \\
\hline 43 & Th-231 & 4.191E-03 \\
\hline 44 & $\operatorname{Ra}-228$ & $7.068 E-01$ \\
\hline 45 & Ac-228 & $1.009 \mathrm{E}-03$ \\
\hline
\end{tabular}




\begin{tabular}{|c|c|c|}
\hline & $\mathbf{B}$ & $\mathrm{O}$ \\
\hline 9 & Nuclide & Weathering Factor (1 year) \\
\hline 10 & Am-241 & $=\left(0.63 /(\$ N 10+\$ N \$ 4)^{*}\left(1-E X P\left(-(\$ N 10+\$ N \$ 4)^{*} \$ R \$ 5\right)\right)\right)+\left((0.37 /(\$ N 10+\$ N \$ 6))^{*}\left(1-E X P\left(-(\$ N 10+\$ N \$ 6)^{*} \$ R \$ 5\right)\right)\right)$ \\
\hline 11 & Am-243 & $=\left(0.63 /(\$ N 11+\$ N \$ 4)^{*}\left(1-E X P\left(-(\$ N 11+\$ N \$ 4)^{*} \$ R \$ 5\right)\right)\right)+\left((0.37 /(\$ N 11+\$ N \$ 6))^{*}\left(1-E X P\left(-(\$ N 11+\$ N \$ 6)^{*} \$ R \$ 5\right)\right)\right)$ \\
\hline 12 & Ba-133 & $=\left(0.63 /(\$ N 12+\$ N \$ 4)^{*}\left(1-\operatorname{EXP}\left(-(\$ N 12+\$ N \$ 4)^{\star} \$ R \$ 5\right)\right)\right)+\left((0.37 /(\$ N 12+\$ N \$ 6))^{*}\left(1-\operatorname{EXP}\left(-(\$ N 12+\$ N \$ 6)^{\star} \$ R \$ 5\right)\right)\right)$ \\
\hline 13 & Ce-144 & $=\left(0.63 /(\$ N 13+\$ N \$ 4)^{*}\left(1-\operatorname{EXP}\left(-(\$ N 13+\$ N \$ 4)^{*} \$ R \$ 5\right)\right)\right)+\left((0.37 /(\$ N 13+\$ N \$ 6))^{*} \cdot\left(1-\operatorname{EXP}\left(-(\$ N 13+\$ N \$ 6)^{*} \$ R \$ 5\right)\right)\right)$ \\
\hline 14 & Cf-249 & $=\left(0.63 /(\$ N 14+\$ N \$ 4)^{*}\left(1-\operatorname{EXP}\left(-(\$ N 14+\$ N \$ 4)^{*} \$ R \$ 5\right)\right)\right)+\left((0.37 /(\$ N 14+\$ N \$ 6))^{*}\left(1-E X P\left(-(\$ N 14+\$ N \$ 6)^{*} \$ R \$ 5\right)\right)\right)$ \\
\hline 15 & Cf-252 & $=\left(0.63 /(\$ N 15+\$ N \$ 4)^{*}\left(1-\operatorname{EXP}\left(-(\$ N 15+\$ N \$ 4)^{*} \$ R \$ 5\right)\right)\right)+\left((0.37 /(\$ N 15+\$ N \$ 6))^{*}\left(1-E X P\left(-(\$ N 15+\$ N \$ 6)^{*} \$ R \$ 5\right)\right)\right)$ \\
\hline 16 & $\mathrm{Cm}-244$ & $=\left(0.63 /(\$ N 16+\$ N \$ 4)^{*}\left(1-E X P\left(-(\$ N 16+\$ N \$ 4)^{*} \$ R \$ 5\right)\right)\right)+\left((0.37 /(\$ N 16+\$ N \$ 6))^{*}\left(1-E X P\left(-(\$ N 16+\$ N \$ 6)^{*} \$ R \$ 5\right)\right)\right)$ \\
\hline 17 & $\mathrm{Cm}-246$ & $=\left(0.63 /(\$ N 17+\$ N \$ 4)^{*}\left(1-E X P\left(-(\$ N 17+\$ N \$ 4)^{*} \$ R \$ 5\right)\right)\right)+\left((0.37 /(\$ N 17+\$ N \$ 6))^{*}\left(1-E X P\left(-(\$ N 17+\$ N \$ 6)^{*} \$ R \$ 5\right)\right)\right)$ \\
\hline 18 & Co-60 & $=\left(0.63 /(\$ N 18+\$ N \$ 4)^{*}\left(1-E X P\left(-(\$ N 18+\$ N \$ 4)^{*} \$ R \$ 5\right)\right)\right)+\left((0.37 /(\$ N 18+\$ N \$ 6))^{*}\left(1-E X P\left(-(\$ N 18+\$ N \$ 6)^{*} \$ R \$ 5\right)\right)\right)$ \\
\hline 19 & Cs-137 & $=\left(0.63 /(\$ N 19+\$ N \$ 4)^{*}\left(1-\operatorname{EXP}\left(-(\$ N 19+\$ N \$ 4)^{*} \$ R \$ 5\right)\right)\right)+\left((0.37 /(\$ N 19+\$ N \$ 6))^{*}\left(1-\operatorname{EXP}\left(-(\$ N 19+\$ N \$ 6)^{*} \$ R \$ 5\right)\right)\right)$ \\
\hline 20 & H-3 & $=\left(0.63 /(\$ N 20+\$ N \$ 4)^{*}\left(1-E X P\left(-(\$ N 20+\$ N \$ 4)^{*} \$ R \$ 5\right)\right)\right)+\left((0.37 /(\$ N 20+\$ N \$ 6))^{*}\left(1-E X P\left(-(\$ N 20+\$ N \$ 6)^{*} \$ R \$ 5\right)\right)\right)$ \\
\hline 21 & Np-237 & $=\left(0.63 /(\$ N 21+\$ N \$ 4)^{*}\left(1-E X P\left(-(\$ N 21+\$ N \$ 4)^{*} \$ R \$ 5\right)\right)\right)+\left((0.37 /(\$ N 21+\$ N \$ 6))^{*}\left(1-E X P\left(-(\$ N 21+\$ N \$ 6)^{*} \$ R \$ 5\right)\right)\right)$ \\
\hline 22 & Pm-147 & $=\left(0.63 /(\$ N 22+\$ N \$ 4)^{*}\left(1-E X P\left(-(\$ N 22+\$ N \$ 4)^{*} \$ R \$ 5\right)\right)\right)+\left((0.37 /(\$ N 22+\$ N \$ 6))^{*}\left(1-E X P\left(-(\$ N 22+\$ N \$ 6)^{*} \$ R \$ 5\right)\right)\right)$ \\
\hline 23 & Pu-238 & $=\left(0.63 /(\$ N 23+\$ N \$ 4)^{*}\left(1-E X P\left(-(\$ N 23+\$ N \$ 4)^{*} \$ R \$ 5\right)\right)\right)+\left((0.37 /(\$ N 23+\$ N \$ 6))^{*}\left(1-E X P\left(-(\$ N 23+\$ N \$ 6)^{*} \$ R \$ 5\right)\right)\right)$ \\
\hline 24 & Pu-239 & $=\left(0.63 /(\$ N 24+\$ N \$ 4)^{*}\left(1-\operatorname{EXP}\left(-(\$ N 24+\$ N \$ 4)^{*} \$ R \$ 5\right)\right)\right)+\left((0.37 /(\$ N 24+\$ N \$ 6))^{*}\left(1-E X P\left(-(\$ N 24+\$ N \$ 6)^{*} \$ R \$ 5\right)\right)\right)$ \\
\hline 25 & Pu mix-239/241 & \\
\hline 26 & Pu-240 & $=\left(0.63 /(\$ N 26+\$ N \$ 4)^{*}\left(1-E X P\left(-(\$ N 26+\$ N \$ 4)^{*} \$ R \$ 5\right)\right)\right)+\left((0.37 /(\$ N 26+\$ N \$ 6))^{*}\left(1-E X P\left(-(\$ N 26+\$ N \$ 6)^{*} \$ R \$ 5\right)\right)\right)$ \\
\hline 27 & Pu-241 & $=\left(0.63 /(\$ N 27+\$ N \$ 4)^{*}\left(1-E X P\left(-(\$ N 27+\$ N \$ 4)^{*} \$ R \$ 5\right)\right)\right)+\left((0.37 /(\$ N 27+\$ N \$ 6))^{*}\left(1-E X P\left(-(\$ N 27+\$ N \$ 6)^{*} \$ R \$ 5\right)\right)\right)$ \\
\hline 28 & Pu pure-242 & $=\left(0.63 /(\$ N 28+\$ N \$ 4)^{*}\left(1-E X P\left(-(\$ N 28+\$ N \$ 4)^{*} \$ R \$ 5\right)\right)\right)+\left((0.37 /(\$ N 28+\$ N \$ 6))^{*}\left(1-E X P\left(-(\$ N 28+\$ N \$ 6)^{*} \$ R \$ 5\right)\right)\right)$ \\
\hline 29 & Sr-90 & $=\left(0.63 /(\$ N 29+\$ N \$ 4)^{*}\left(1-E X P\left(-(\$ N 29+\$ N \$ 4)^{*} \$ R \$ 5\right)\right)\right)+\left((0.37 /(\$ N 29+\$ N \$ 6))^{*}\left(1-E X P\left(-(\$ N 29+\$ N \$ 6)^{*} \$ R \$ 5\right)\right)\right)$ \\
\hline 30 & Tc-99 & $=\left(0.63 /(\$ N 30+\$ N \$ 4)^{*}\left(1-\operatorname{EXP}\left(-(\$ N 30+\$ N \$ 4)^{\star} \$ R \$ 5\right)\right)\right)+\left((0.37 /(\$ N 30+\$ N \$ 6))^{*}\left(1-E X P\left(-(\$ N 30+\$ N \$ 6)^{*} \$ R \$ 5\right)\right)\right)$ \\
\hline 31 & Th-232 & $=\left(0.63 /(\$ N 31+\$ N \$ 4)^{*}\left(1-\operatorname{EXP}\left(-(\$ N 31+\$ N \$ 4)^{*} \$ R \$ 5\right)\right)\right)+\left((0.37 /(\$ N 31+\$ N \$ 6))^{*}\left(1-\operatorname{EXP}\left(-(\$ N 31+\$ N \$ 6)^{*} \$ R \$ 5\right)\right)\right)$ \\
\hline 32 & $\mathrm{TI}-204$ & $=\left(0.63 /(\$ N 32+\$ N \$ 4)^{*}\left(1-\operatorname{EXP}\left(-(\$ N 32+\$ N \$ 4)^{*} \$ R \$ 5\right)\right)\right)+\left((0.37 /(\$ N 32+\$ N \$ 6))^{*}\left(1-\operatorname{EXP}\left(-(\$ N 32+\$ N \$ 6)^{*} \$ R \$ 5\right)\right)\right)$ \\
\hline 33 & $U-235$ & $=\left(0.63 /(\$ N 33+\$ N \$ 4)^{*}\left(1-\operatorname{EXP}\left(-(\$ N 33+\$ N \$ 4)^{*} \$ R \$ 5\right)\right)\right)+\left((0.37 /(\$ N 33+\$ N \$ 6))^{*}\left(1-E X P\left(-(\$ N 33+\$ N \$ 6)^{*} \$ R \$ 5\right)\right)\right)$ \\
\hline 34 & $\mathrm{U}-238$ & $=\left(0.63 /(\$ N 34+\$ N \$ 4)^{\star}\left(1-E X P\left(-(\$ N 34+\$ N \$ 4)^{*} \$ R \$ 5\right)\right)\right)+\left((0.37 /(\$ N 34+\$ N \$ 6))^{*}\left(1-E X P\left(-(\$ N 34+\$ N \$ 6)^{*} \$ R \$ 5\right)\right)\right)$ \\
\hline 35 & Total & \\
\hline 36 & $\mathrm{Ba}-137 \mathrm{~m}$ & $=\left(0.63 /(\$ N 36+\$ N \$ 4)^{*}\left(1-\operatorname{EXP}\left(-(\$ N 36+\$ N \$ 4)^{*} \$ R \$ 5\right)\right)\right)+\left((0.37 /(\$ N 36+\$ N \$ 6))^{*}\left(1-E X P\left(-(\$ N 36+\$ N \$ 6)^{*} \$ R \$ 5\right)\right)\right)$ \\
\hline 37 & $Y-90$ & $=\left(0.63 /(\$ N 37+\$ N \$ 4)^{*}\left(1-\operatorname{EXP}\left(-(\$ N 37+\$ N \$ 4)^{*} \$ R \$ 5\right)\right)\right)+\left((0.37 /(\$ N 37+\$ N \$ 6))^{*}\left(1-E X P\left(-(\$ N 37+\$ N \$ 6)^{*} \$ R \$ 5\right)\right)\right)$ \\
\hline 38 & Pr-144 & $=\left(0.63 /(\$ N 38+\$ N \$ 4)^{*}\left(1-E X P\left(-(\$ N 38+\$ N \$ 4)^{*} \$ R \$ 5\right)\right)\right)+\left((0.37 /(\$ N 38+\$ N \$ 6))^{*}\left(1-E X P\left(-(\$ N 38+\$ N \$ 6)^{*} \$ R \$ 5\right)\right)\right)$ \\
\hline 39 & Pa-233 & $=\left(0.63 /(\$ N 39+\$ N \$ 4)^{*}\left(1-\operatorname{EXP}\left(-(\$ N 39+\$ N \$ 4)^{*} \$ R \$ 5\right)\right)\right)+\left((0.37 /(\$ N 39+\$ N \$ 6))^{\star}\left(1-E X P\left(-(\$ N 39+\$ N \$ 6)^{*} \$ R \$ 5\right)\right)\right)$ \\
\hline 40 & Th-234 & $=\left(0.63 /(\$ N 40+\$ N \$ 4)^{*}\left(1-E X P\left(-(\$ N 40+\$ N \$ 4)^{*} \$ R \$ 5\right)\right)\right)+\left((0.37 /(\$ N 40+\$ N \$ 6))^{*}\left(1-E X P\left(-(\$ N 40+\$ N \$ 6)^{*} \$ R \$ 5\right)\right)\right)$ \\
\hline 41 & Pa-234 & $=\left(0.63 /(\$ N 41+\$ N \$ 4)^{*}\left(1-E X P\left(-(\$ N 41+\$ N \$ 4)^{*} \$ R \$ 5\right)\right)\right)+\left((0.37 /(\$ N 41+\$ N \$ 6))^{*}\left(1-E X P\left(-(\$ N 41+\$ N \$ 6)^{*} \$ R \$ 5\right)\right)\right)$ \\
\hline 42 & Np-239 & $=\left(0.63 /(\$ N 42+\$ N \$ 4)^{*}\left(1-\operatorname{EXP}\left(-(\$ N 42+\$ N \$ 4)^{*} \$ R \$ 5\right)\right)\right)+\left((0.37 /(\$ N 42+\$ N \$ 6))^{*}\left(1-E X P\left(-(\$ N 42+\$ N \$ 6)^{*} \$ R \$ 5\right)\right)\right)$ \\
\hline 43 & Th-231 & $=\left(0.63 /(\$ N 43+\$ N \$ 4)^{*}\left(1-E X P\left(-(\$ N 43+\$ N \$ 4)^{*} \$ R \$ 5\right)\right)\right)+\left((0.37 /(\$ N 43+\$ N \$ 6))^{*}\left(1-E X P\left(-(\$ N 43+\$ N \$ 6)^{\star} \$ R \$ 5\right)\right)\right)$ \\
\hline 44 & Ra-228 & $=\left(0.63 /(\$ N 44+\$ N \$ 4)^{*}\left(1-E X P\left(-(\$ N 44+\$ N \$ 4)^{*} \$ R \$ 5\right)\right)\right)+\left((0.37 /(\$ N 44+\$ N \$ 6))^{*}\left(1-E X P\left(-(\$ N 44+\$ N \$ 6)^{\star} \$ R \$ 5\right)\right)\right)$ \\
\hline 45 & Ac-228 & $=\left(0.63 /(\$ N 45+\$ N \$ 4)^{\star}\left(1-\operatorname{EXP}\left(-(\$ N 45+\$ N \$ 4)^{\star} \$ R \$ 5\right)\right)\right)+\left((0.37 /(\$ N 45+\$ N \$ 6))^{*}\left(1-E X P\left(-(\$ N 45+\$ N \$ 6)^{*} \$ R \$ 5\right)\right)\right)$ \\
\hline
\end{tabular}




\begin{tabular}{|l|ll|}
\hline & $\mathrm{F}$ & \multicolumn{1}{c|}{} \\
\hline 52 & $1.46 E+01$ & DRL (uCi/m^2) - PAG/(CEDE+External) \\
\cline { 1 - 1 } 53 & $1.12 E+01$ & DRL (uCi/m^2) - PAG/(BS Dose + External) \\
\cline { 1 - 1 } 54 & $1.72 E+03$ & DRL (uCi/m^2) - PAG/(Skin Dose) \\
\hline
\end{tabular}




\begin{tabular}{|c|c|c|}
\hline & $F$ & $\bar{H}$ \\
\hline 52 & $=\mathrm{H} 3 /(\mathrm{H} 47+147)$ & DRL (uCi/m^2) - PAG/(CEDE+External) \\
\hline 53 & $=H 4 /(L 47+147)$ & DRL (uCi/m^2) - PAG/(BS Dose + External) \\
\hline 54 & $=\mathrm{H} 5 / \mathrm{K} 47$ & DRL (uCi/m^2) - PAG/(Skin Dose) \\
\hline
\end{tabular}




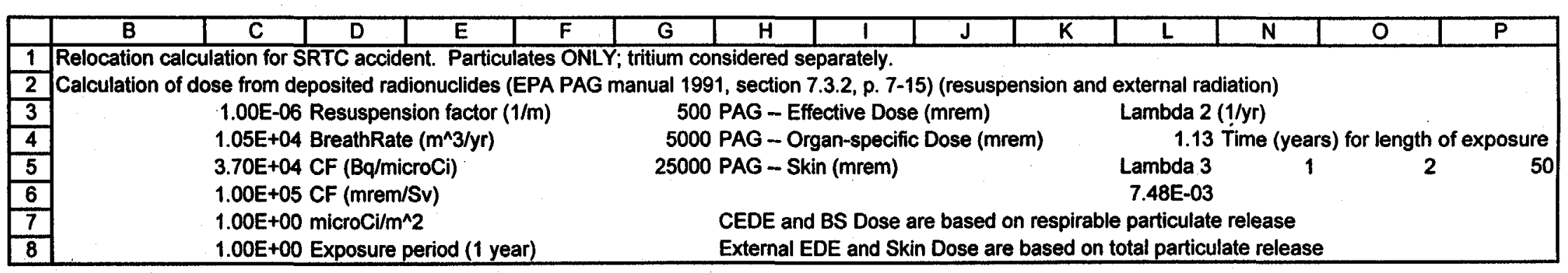




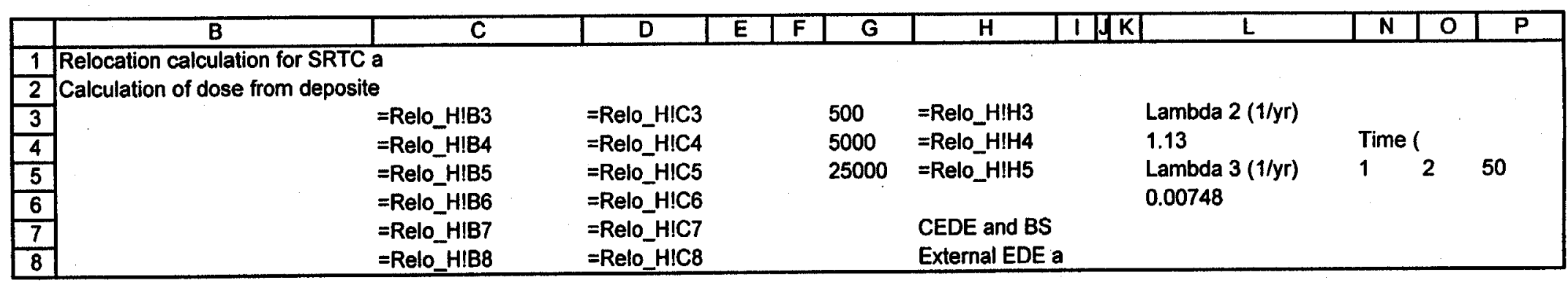




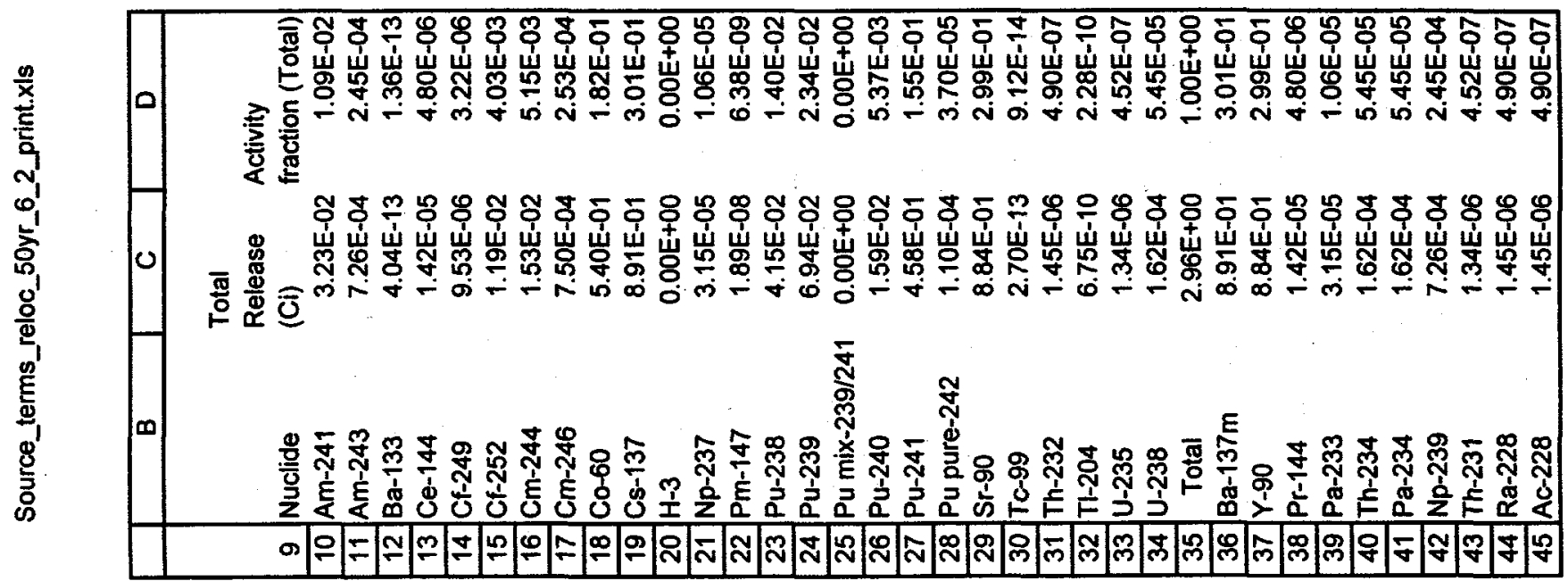




\begin{tabular}{|c|c|c|c|}
\hline & $\mathbf{B}$ & $\mathbf{C}$ & D \\
\hline 9 & Nuclide & Total Release (Ci) & $\begin{array}{l}\text { Activity fraction } \\
\text { (Total) }\end{array}$ \\
\hline 10 & Am-241 & ='C:IEP_relocationVSourceSRTC.xls]resp sums' $\$$ \$H10 & $=\mathrm{C} 10 / \$ \mathrm{C} \$ 35$ \\
\hline 11 & Am-243 & ='C:IEP_relocationYSourceSRTC.xIs]resp sums'I\$H11 & $=C 11 / \$ C \$ 35$ \\
\hline 12 & Ba-133 & $=' C: I E P$ relocation丩SourceSRTC.xls]resp sums'!\$H12 & $=\mathrm{C} 12 / \$ \mathrm{C} \$ 35$ \\
\hline 13 & $\mathrm{Ce}-144$ & $={ }^{\prime} C:$ :EP_relocation $[S o u r c e S R T C . x \mid s]$ resp sums $! \$ H 13$ & $=\mathrm{C} 13 / \$ C \$ 35$ \\
\hline 14 & Cf-249 & ='C:IEP_relocationYSourceSRTC.xIs]resp sums'!\$H14 & $=\mathrm{C} 14 / \$ \mathrm{C} \$ 35$ \\
\hline 15 & Cf-252 & ='C:IEP_relocationVSourceSRTC.xls]resp sums'!\$H15 & $=\mathrm{C} 15 / \$ C \$ 35$ \\
\hline 16 & $\mathrm{Cm}-244$ & $=' C: I E P \_$relocationYSourceSRTC.xIs]resp sums'I\$H16 & $=\mathrm{C} 16 / \$ C \$ 35$ \\
\hline 17 & $\mathrm{Cm}-246$ & ='C:IEP_relocationYSourceSRTC.xls]resp sums'I\$H17 & $=C 17 / \$ C \$ 35$ \\
\hline 18 & Co-60 & $={ }^{\prime} C:$ IEP_relocationy[SourceSRTC.xis]resp sums'!\$H18 & $=\mathrm{C} 18 / \$ \mathrm{C} \$ 35$ \\
\hline 19 & Cs-137 & ='C:IEP_relocation YSourceSRTC.xIs]resp sums'I\$H19 & $=\mathrm{C} 19 / \$ \mathrm{C} \$ 35$ \\
\hline 20 & $\mathrm{H}-3$ & 0 & $=\mathrm{C} 20 / \$ C \$ 35$ \\
\hline 21 & $\mathrm{~Np}-237$ & $={ }^{\prime} \mathrm{C}: \mathrm{IEP}$ _relocationySourceSRTC.xIs]resp sums'!\$H21 & $=\mathrm{C} 21 / \$ \mathrm{C} \$ 35$ \\
\hline 22 & Pm-147 & $=' C:$ IEP_relocation $[$ SourceSRTC.x|s]resp sums'!\$H22 & $=\mathrm{C} 22 / \$ \mathrm{C} \$ 35$ \\
\hline 23 & Pu-238 & ='C:IEP_relocationVSourceSRTC.x|s]resp sums'!\$H23 & $=\mathrm{C} 23 / \$ \mathrm{C} \$ 35$ \\
\hline 24 & Pu-239 & $=$ ='C:IEP_relocationYSourceSRTC.xIs]resp sums'!\$H24 & $=\mathrm{C} 24 / \$ C \$ 35$ \\
\hline 25 & Pu mix-239/241 & $=' C: \mid E P \_$relocationYSourceSRTC.xls]resp sums'I\$H25 & $=\mathrm{C} 25 / \$ C \$ 35$ \\
\hline 26 & Pu-240 & ='C:IEP_relocationYSourceSRTC.xIs]resp sums'!\$H26 & $=\mathrm{C} 26 / \$ C \$ 35$ \\
\hline 27 & Pu-241 & $=' C:$ IEP_relocation $[$ SourceSRTC.xls]resp sums'!\$H27 & $=\mathrm{C} 27 / \$ \mathrm{C} \$ 35$ \\
\hline 28 & Pu pure-242 & ='C:IEP_relocationUSourceSRTC.xls]resp sums'!\$H28 & $=\mathrm{C} 28 / \$ C \$ 35$ \\
\hline 29 & Sr-90 & $={ }^{\prime}$ C:IEP_relocationVSourceSRTC.xIs]resp sums $\mid \$ H 29$ & $=\mathrm{C} 29 / \$ C \$ 35$ \\
\hline 30 & Tc-99 & $=' C:$ IEP_relocation $\backslash$ SourceSRTC.xis]resp sums' $1 \$ \mathrm{H} 30$ & $=\mathrm{C} 30 / \$ C \$ 35$ \\
\hline 31 & Th-232 & $=$ ='C:IEP_relocationYSourceSRTC.xIs]resp sums'!\$H31 & $=\mathrm{C} 31 / \mathrm{C} \$ 35$ \\
\hline 32 & TI-204 & ='C:IEP_relocationYSourceSRTC.xIs]resp sums'I\$H32 & $=\mathrm{C} 32 / \$ \mathrm{C} \$ 35$ \\
\hline 33 & U-235 & ='C:IEP_relocationV(SourceSRTC.xIs]resp sums'!\$H33 & $=\mathrm{C} 33 / \$ C \$ 35$ \\
\hline 34 & $\mathrm{U}-238$ & $=$ 'C:IEP_relocationVSourceSRTC.xIs]resp sums'!\$H34 & $=\mathrm{C} 34 / \$ \mathrm{C} \$ 35$ \\
\hline 35 & Total & $=\operatorname{SUM}(\overline{\mathrm{C}} 10: \mathrm{C} 34)$ & $=S U M(D 10: D 34)$ \\
\hline 36 & Ba-137m & $=\mathrm{C} 19$ & $=\mathrm{D} 19$ \\
\hline 37 & $Y-90$ & $=\mathrm{C} 29$ & $=\mathrm{D} 29$ \\
\hline 38 & Pr-144 & $=\mathrm{C} 13$ & $=\mathrm{D} 13$ \\
\hline 39 & Pa-233 & $=\mathrm{C} 21$ & $=\mathrm{D} 21$ \\
\hline 40 & Th-234 & $=C 34$ & $=\mathrm{D} 34$ \\
\hline 41 & $\mathrm{~Pa}-234$ & $=C 34$ & $=\mathrm{D} 34$ \\
\hline 42 & $\mathrm{~Np}-239$ & $=\mathrm{C} 11$ & $=011$ \\
\hline 43 & Th-231 & $=\mathrm{C} 33$ & $=\mathrm{D} 33$ \\
\hline 44 & Ra-228 & $=\mathrm{C} 31$ & $=\mathrm{D} 31$ \\
\hline 45 & $A C-228$ & $=\mathrm{C} 31$ & $=\mathrm{D} 31$ \\
\hline
\end{tabular}




\begin{tabular}{|c|c|c|c|}
\hline & B & $\bar{E}$ & $\mathbf{F}$ \\
\hline 9 & Nuclide ( & $\begin{array}{l}\text { Respirable Release' } \\
\text { (Ci) }\end{array}$ & $\begin{array}{l}\text { Activity fraction } \\
\text { (Resp) }\end{array}$ \\
\hline 10 & Am-241 & $1.49 E-02$ & 5.56E-03 \\
\hline 11 & Am-243 & 4.55E-04 & 1.70E-04 \\
\hline 12 & $\mathrm{Ba}-133$ & $4.04 E-13$ & $1.51 \mathrm{E}-13$ \\
\hline 13 & $\mathrm{Ce}-144$ & $1.42 E-05$ & 5.32E-06 \\
\hline$\overline{14}$ & Cf-249 & $8.24 E-06$ & 3.08E-06 \\
\hline 15 & Cf-252 & 1.19E-02 & 4.47E-03 \\
\hline 16 & $\mathrm{Cm}-244$ & 1.71E-02 & $6.40 E-03$ \\
\hline 17 & $\mathrm{Cm}-246$ & 4.99E-04 & $1.87 E-04$ \\
\hline 18 & Co-60 & $5.40 E-01$ & $2.02 E-01$ \\
\hline 19 & Cs-137 & 8.91E-01 & 3.34E-01 \\
\hline 20 & $H-3$ & $0.00 \mathrm{E}+00$ & $0.00 E+00$ \\
\hline 21 & Np-237 & 2.44E-05 & 9.14E-06 \\
\hline 22 & $\mathrm{Pm}-147$ & $1.89 E-08$ & 7.07E-09 \\
\hline 23 & Pu-238 & $1.81 \mathrm{E}-02$ & 6.77E-03 \\
\hline 24 & $P u-239$ & 2.35E-02 & 8.78E-03 \\
\hline 25 & Pu mix-2 & $0.00 E+00$ & $0.00 E+00$ \\
\hline 26 & Pu-240 & 5.34E-03 & 2.00E-03 \\
\hline 27 & $P u-241$ & 2.66E-01 & 9.97E-02 \\
\hline 28 & Pu pure- & 3.47E-05 & 1.30E-05 \\
\hline 29 & Sr-90 & 8.83E-01 & 3.30E-01 \\
\hline 30 & Tc-99 & $2.70 E-13$ & $1.01 E-13$ \\
\hline 31 & Th-232 & 1.44E-06 & 5.38E-07 \\
\hline 32 & TI-204 & $6.75 \mathrm{E}-10$ & 2.53E-10 \\
\hline 33 & U-235 & $6.88 \mathrm{E}-07$ & 2.57E-07 \\
\hline 34 & U-238 & 5.11E-05 & 1.91E-05 \\
\hline 35 & Total & $2.67 E+00$ & $1.00 E+00$ \\
\hline 36 & $\mathrm{Ba}-137 \mathrm{~m}$ & 8.91E-01 & 3.34E-01 \\
\hline 37 & $Y-90$ & 8.83E-01 & 3.30E-01 \\
\hline 38 & Pr-144 & 1.42E-05 & 5.32E-06 \\
\hline 39 & Pa-233 & 2.44E-05 & $9.14 E-06$ \\
\hline 40 & Th-234 & 5.11E-05 & 1.91E-05 \\
\hline 41 & Pa-234 & 5.11E-05 & 1.91E-05 \\
\hline 42 & $\mathrm{~Np}-239$ & 4.55E-04 & 1.70E-04 \\
\hline 43 & Th-231 & $6.88 \mathrm{E}-07$ & 2.57E-07 \\
\hline 44 & Ra-228 & $1.44 E-06$ & 5.38E-07 \\
\hline 45 & Ac-228 & $1.44 \mathrm{E}-06$ & 5.38E-07 \\
\hline
\end{tabular}




\begin{tabular}{|c|c|c|c|}
\hline & $\bar{B}$ & $\mathbf{E}$ & $\bar{F}$ \\
\hline & & & . \\
\hline 9 & Nuclide & Respirable Release (Ci) & Activity fraction (Resp) \\
\hline 10 & Am-241 & $=$ 'C:IEP_relocation \SourceSRTC.xis\}resp sums'!\$F10 & $=\mathrm{E} 10 / \$ \mathrm{E} \$ 35$ \\
\hline 11 & Am-243 & $=' C$ :IEP_relocation YSourceSRTC.xIs]resp sums'!\$F11 & $=E 11 / \$ E \$ 35$ \\
\hline 12 & Ba-133 & $=' C$ :IEP_relocation YSourceSRTC.x|s]resp sums'!\$F12 & $=E 12 / \$ E \$ 35$ \\
\hline 13 & $\mathrm{Ce}-144$ & $=$ 'C:IEP_relocationYSourceSRTC.xIs]resp sums' $1 \$ F 13$ & $=E 13 / \$ E \$ 35$ \\
\hline 14 & Cf-249 & $=$ 'C:IEP_relocationYSourceSRTC.xIs]resp sums'I\$F14 & $=E 14 / \$ E \$ 35$ \\
\hline 15 & Cf-252 & $=' C$ :IEP_relocationYSourceSRTC.xIs]resp sums'I\$F 15 & $=\mathrm{E} 15 / \$ E \$ 35$ \\
\hline 16 & $\mathrm{Cm}-244$ & $={ }^{\prime} \mathrm{C}: \mathrm{IEP}$ _relocation YSourceSRTC.xIs]resp sums'!\$F16 & $=\mathrm{E} 16 / \$ \mathrm{E} \$ 35$ \\
\hline 17 & $\mathrm{Cm}-246$ & $=$ 'C:IEP_relocationYSourceSRTC.xls]resp sums'I\$F17 & $=E 17 / \$ E \$ 35$ \\
\hline 18 & Co-60 & $={ }^{\prime} \mathrm{C}:$ IEP_relocation YSourceSRTC.xIs]resp sums'!\$F18 & $=\mathrm{E} 18 / \$ E \$ 35$ \\
\hline 19 & Cs-137 & ='C:IEP_relocationYSourceSRTC.xIs]resp sums'!\$F19 & $=E 19 / \$ E \$ 35$ \\
\hline 20 & $\mathrm{H}-3$ & 0 & $=E 20 / \$ E \$ 35$ \\
\hline 21 & Np-237 & $=' C$ :IEP_relocationUSourceSRTC.xls]resp sums' $\$ F 21$ & $=\mathrm{E} 21 / \$ \mathrm{E} \$ 35$ \\
\hline 22 & Pm-147 & $={ }^{\prime} \mathrm{C}:$ IEP_relocation $\backslash$ SourceSRTC.xIs]resp sums' $\$$ \$F22 & $=E 22 / \$ E \$ 35$ \\
\hline 23 & Pu-238 & $={ }^{\prime}$ C:IEP_relocationYSourceSRTC.xIs]resp sums'!\$F23 & $=E 23 / \$ E \$ 35$ \\
\hline 24 & Pu-239 & $=' C$ :IEP_relocation\[SourceSRTC.x|s]resp sums'I\$F24 & $=E 24 / \$ E \$ 35$ \\
\hline 25 & Pu mix-239/241 & $={ }^{\prime} \mathrm{C}$ :IEP_relocationYSourceSRTC.xis]resp sums'I\$F25 & $=E 25 / \$ E \$ 35$ \\
\hline 26 & Pu-240 & $={ }^{\prime}$ C:IEP_relocationYSourceSRTC.xis]resp sums'!\$F26 & $=E 26 / \$ E \$ 35$ \\
\hline 27 & Pu-241 & $=' C:$ IEP_relocationYSourceSRTC.xIs]resp sums' $1 \$ F 27$ & $=E 27 / \$ E \$ 35$ \\
\hline 28 & Pu pure-242 & 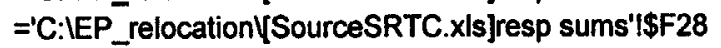 & $=E 28 / \$ E \$ 35$ \\
\hline 29 & Sr-90 & ='C:IEP_relocationYSourceSRTC.xIs]resp sums'!\$F29 & $=E 29 / \$ E \$ 35$ \\
\hline 30 & Tc-99 & $=' C$ :IEP_relocationY̧SourceSRTC.XIs]resp sums'!\$F30 & $=E 30 / \$ E \$ 35$ \\
\hline 31 & Th-232 & $=$ C:IEP_relocation YSourceSRTC.xIs]resp sums'!\$F31 & $=\mathrm{E} 31 /$ \$E\$35 \\
\hline 32 & TI-204 & ='C:IEP_relocationYSourceSRTC.xIs]resp sums'I\$F32 & $=E 32 / \$ E \$ 35$ \\
\hline 33 & U-235 & $=' C$ :IEP_relocation\[SourceSRTC.XIs]resp sums'!\$F33 & $=E 33 / \$ E \$ 35$ \\
\hline 34 & $\mathrm{U}-238$ & ='C:IEP_relocationVSourceSRTC.xIs]resp sums'I\$F34 & $=E 34 / \$ E \$ 35$ \\
\hline 35 & Total & $=S U M(\bar{E} 10: E 34)$ & $=$ SUM(F10:F34) \\
\hline 36 & $\mathrm{Ba}-137 \mathrm{~m}$ & $=\mathrm{E} 19$ & $=F 19$ \\
\hline 37 & $Y-90$ & $=E 29$ & $=F 29$ \\
\hline 38 & Pr-144 & $=E 13$ & $=F 13$ \\
\hline 39 & Pa-233 & $=E 21$ & $=F 21$ \\
\hline 40 & Th-234 & $=E 34$ & $=F 34$ \\
\hline 41 & Pa-234 & $=E 34$ & $=F 34$ \\
\hline 42 & Np-239 & $=E 11$ & $=F 11$ \\
\hline 43 & Th-231 & $=E 33$ & $=F 33$ \\
\hline 44 & Ra-228 & $=E 31$ & $=F 31$ \\
\hline 45 & AC-228 & $=E 31$ & $=F 31$ \\
\hline
\end{tabular}




\begin{tabular}{|c|c|c|c|c|}
\hline & & 3 & $\mathrm{H}$ & $T$ \\
\hline 9 & Nuclide & & $\begin{array}{l}\text { CEDE (mrem per } \\
\mathrm{uCl} / \mathrm{m}^{\wedge} 2 \text { ) }\end{array}$ & 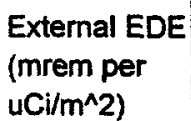 \\
\hline 10 & Am-241 & & $3.20 E+01$ & 4.46E-02 \\
\hline 11 & Am-243 & & 9.72E-01 & 2.22E-03 \\
\hline 12 & $\mathrm{Ba}-133$ & & $1.44 E-14$ & $7.38 E-12$ \\
\hline 13. & $\mathrm{Ce}-144$ & & 1.36E-05 & 7.47E-06 \\
\hline 14 & Cf-249 & & $2.30 E-02$ & $1.55 \mathrm{E}-0.4$ \\
\hline 15 & $\mathrm{C}-252$ & • & $7.34 E+00$ & 2.82E-04 \\
\hline 16 & $\mathrm{Cm}-244$ & & $1.99 E+01$ & $5.65 E-0.4$ \\
\hline 17 & $\mathrm{Cm}-246$ & & $1.09 E+00$ & 2.54E-05 \\
\hline 18 & Co-60 & & 5.12E-01 & 5.07E+01 \\
\hline 19 & Cs-137 & & 1.35E-01 & $0.00 E+\infty$ \\
\hline 20 & $H-3$ & & $0.00 E+00$ & $0.00 E+00$ \\
\hline 21 & $\mathrm{~Np}-237$ & & $6.40 E-02$ & 4.71E-05 \\
\hline 22 & Pm-147 & & 2.90E-09 & 2.88E-12 \\
\hline 23 & $\mathrm{Pu}-238$ & & $3.42 E+01$ & $1.63 E-03$ \\
\hline 24 & Pu-239 & & $4.88 \mathrm{E}+01$ & 1.21E-03 \\
\hline 25 & Pu mix-239/241 & & $0.00 E+00$ & $0.00 E+00$ \\
\hline 26 & Pu-240 & & $1.11 E+01$ & $6.02 E-04$ \\
\hline 27 & Pu-241 & & $1.02 E+01$ & $0.00 E+00$ \\
\hline 28 & Pu pure-242 & & 6.91E-02 & $3.45 E-06$ \\
\hline 29 & Sr-90 & & $5.44 E+\infty$ & $0.00 E+00$ \\
\hline 30 & Tc-99 & & $1.09 E-14$ & $7.80 \mathrm{E}-18$ \\
\hline 31 & Th-232 & & $1.14 E-02$ & 4.46E-08 \\
\hline 32 & TI-204 & & 6.77E-12 & $3.48 \mathrm{E}-11$ \\
\hline 33 & U-235 & & $4.09 E-04$ & $1.06 \mathrm{E}-05$ \\
\hline 34 & $U-238$ & & 2.93E-02 & 4.82E-06 \\
\hline 35 & Total & & & \\
\hline 36 & $B a-137 m$ & & & $1.43 E-04$ \\
\hline 37 & $Y-90$ & & & $0.00 E+00$ \\
\hline 38 & Pr-144 & & & $7.52 \mathrm{E}-10$ \\
\hline 39 & $\mathrm{~Pa}-233$ & & & 2.77E-05 \\
\hline 40 & Th-234 & & & $5.46 \mathrm{E}-06$ \\
\hline 41 & $\mathrm{~Pa}-234$ & & & 1.31E-05 \\
\hline 42 & $\mathrm{~Np}-239$ & & & 4.82E-05 \\
\hline 43 & Th-231 & & & 4.01E-09 \\
\hline 44 & Ra-228 & & & 4.04E-14 \\
\hline 45 & $A C-228$ & & & 4.98E-08 \\
\hline 46 & 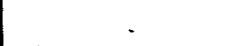 & & & \\
\hline 47 & Total & & $1.72 E+02$ & $5.07 E+01$ \\
\hline 48 & & & & \\
\hline 49 & Dose in secor & ear following release: & 6.70E+01 & $1.84 E+01$ \\
\hline
\end{tabular}




\begin{tabular}{|c|c|c|c|}
\hline & $\bar{B}$ & $\mathrm{H}$ & 1 \\
\hline 9 & Nuclide & CEDE (mrem per $\left.u C l / m^{\wedge} 2\right)$ & External EDE (mrem per uCi/m^2) \\
\hline 10 & Am-241 & $=\$ C \$ 7^{*} \$ C \$ 3^{*} \$ C \$ 4^{*} \$ C \$ 5^{*} \$ C \$ 6^{*} F 10^{*}$ Relocation!H10*P10 & $=\$ C \$ 7^{*} \$ D 10^{*}$ Relocation!E10*\$C $\$ 35 / \$ E \$ 35^{*} \mathrm{P} 10$ \\
\hline 11 & $A m-243$ & $=\$ C \$ 7^{*} \$ C \$ 3^{*} \$ C \$ 4^{*} \$ C \$ 5^{*} \$ C \$ 6^{*}$ F11* Relocation!H11*P11 & $=\$ C \$ 7^{*}$ \$D11*Relocation!E11*\$C $\$ 35 / \$ E \$ 35 * P 11$ \\
\hline 12 & $\mathrm{Ba}-133$ & $=\$ C \$ 7^{*} \$ C \$ 3^{*} \$ C \$ 4^{*} \$ C \$ 5^{*} \$ C \$ 6^{*} F 12^{*}$ Relocation!H12*P12 & $=\$ C \$ 7^{*} \$ D 12^{*}$ Relocation!E12*\$C $\$ 35 / \$ E \$ 35^{*} \mathrm{P} 12$ \\
\hline 13 & $\mathrm{Ce}-144$ & $=\$ C \$ 7^{*} \$ C \$ 3^{*} \$ C \$ 4^{*} \$ C \$ 5^{*} \$ C \$ 6^{*} F 13^{*}$ Relocation!H13*P13 & $=\$ C \$ 7^{*} \$ D 13^{*}$ Relocation!E13*\$C $\$ 35 / \$ E \$ 35^{*} \mathrm{P} 13$ \\
\hline 14 & Cf-249 & $=\$ C \$ 7^{*} \$ C \$ 3^{*} \$ C \$ 4^{*} \$ C \$ 5^{*} \$ C \$ 6^{*} F 14^{*}$ Relocation!H14* $\mathrm{P} 14$ & $=\$ C \$ 7^{*} \$ D 14^{*}$ Relocation!E14*\$C $\$ 35 / \$ E \$ 35^{*} P 14$ \\
\hline 15 & Cf-252 & $=\$ C \$ 7^{*} \$ C \$ 3^{*} \$ C \$ 4^{*} \$ C \$ 5^{*} \$ C \$ 6^{*} F 15^{*}$ Relocation!H15*P15 & $=\$ C \$ 7^{*} \$ D 15^{*}$ Relocation!E15*\$C $\$ 35 / \$ E \$ 35^{*} P 15$ \\
\hline 16 & $\mathrm{Cm}-244$ & $=\$ C \$ 7^{*} \$ C \$ 3^{*} \$ C \$ 4^{*} \$ C \$ 5^{*} \$ C \$ 6^{*} F 16^{*}$ Relocation!H16*P16 & $=\$ C \$ 7^{*} \$ D 16^{*}$ Relocation!E16*\$C $\$ 35 / \$ E \$ 35^{*} P 16$ \\
\hline 17 & $\mathrm{Cm}-246$ & $=\$ C \$ 7^{*} \$ C \$ 3^{*} \$ C \$ 4^{*} \$ C \$ 5^{*} \$ C \$ 6^{*} F 17^{*}$ Relocation!H17*P17 & $=\$ C \$ 7^{*} \$ D 17^{*}$ Relocation!E17*\$C $\$ 35 / \$ E \$ 35^{*} \mathrm{P} 17$ \\
\hline 18 & Co-60 & $=\$ C \$ 7^{*} \$ C \$ 3^{*} \$ C \$ 4^{*} \$ C \$ 5^{*} \$ C \$ 6^{*} F 18^{*}$ Relocation!H18*P18 & $=\$ C \$ 7^{*} \$ D 18^{*}$ RelocationIE18*\$C $\$ 35 / \$ E \$ 35^{*} \mathrm{P} 18$ \\
\hline 19 & Cs-137 & $=\$ C \$ 7^{*} \$ C \$ 3^{*} \$ C \$ 4^{*} \$ C \$ 5^{*} \$ C \$ 6^{*} F 19^{*}$ Relocation!H19*P19 & $=\$ C \$ 7^{*} \$ D 19 *$ Relocation!E19*\$C\$35/\$E\$35*P19 \\
\hline 20 & $\mathrm{H}-3$ & $=\$ C \$ 7^{*} \$ C \$ 3^{*} \$ C \$ 4^{*} \$ C \$ 5^{*} \$ C \$ 6^{*} F 20^{*}$ Relocation!H20*P20 & $=\$ C \$ 7^{*} \$ D 20^{*}$ Relocation!E20*\$C $\$ 35 / \$ E \$ 35 * P 20$ \\
\hline 21 & Np-237 & $=\$ C \$ 7^{*} \$ C \$ 3^{*} \$ C \$ 4^{*} \$ C \$ 5^{*} \$ C \$ 6^{*} F 21^{*}$ Relocation!H21*P21 & $=\$ C \$ 7^{*} \$ D 21^{*}$ Relocation!E21*\$C $\$ 35 / \$ E \$ 35^{*} \mathrm{P} 21$ \\
\hline 22 & Pm-147 & $=\$ C \$ 7^{*} \$ C \$ 3^{*} \$ C \$ 4^{*} \$ C \$ 5^{*} \$ C \$ 6^{*} \mathrm{~F} 22^{*}$ Relocation!H22*P22 & $=\$ C \$ 7^{*} \$ D 22^{*}$ Relocation!E22*\$C $\$ 35 / \$ E \$ 35^{*} P 22$ \\
\hline 23 & Pu-238 & $=\$ C \$ 7^{*} \$ C \$ 3^{*} \$ C \$ 4^{*} \$ C \$ 5^{*} \$ C \$ 6^{*} F 23^{*}$ Relocation!H23*P23 & $=\$ C \$ 7^{*} \$ D 23^{*}$ Relocation!E23*\$C\$35/\$E $\$ 35^{*} \mathrm{P} 23$ \\
\hline 24 & Pu-239 & $=\$ C \$ 7^{*} \$ C \$ 3^{*} \$ C \$ 4^{*} \$ C \$ 5^{*} \$ C \$ 6^{*} F 24^{*}$ Relocation!H24*P24 & $=\$ C \$ 7^{*} \$ D 24^{*}$ Relocation!E24*\$C $\$ 35 / \$ E \$ 35^{*} P 24$ \\
\hline 25 & Pu mix-239/241 & $=\$ C \$ 7^{*} \$ C \$ 3^{*} \$ C \$ 4^{*} \$ C \$ 5^{*} \$ C \$ 6^{*} F 25^{*}$ Relocation!H25*25 & $=\$ C \$ 7^{*} \$ D 25^{*}$ Relocation!E25*\$C\$35/\$E\$35*P25 \\
\hline 26 & Pu-240 & $=\$ C \$ 7^{*} \$ C \$ 3^{*} \$ C \$ 4^{*} \$ C \$ 5^{*} \$ C \$ 6^{*} F 26^{*}$ Relocation!H26*26 & $=\$ C \$ 7^{*} \$ D 26^{*}$ Relocation!E26*\$C $\$ 35 / \$ E \$ 35^{*} P 26$ \\
\hline 27 & Pu-241 & $=\$ C \$ 7^{*} \$ C \$ 3^{*} \$ C \$ 4^{*} \$ C \$ 5^{*} \$ C \$ 6^{*} F 27^{*}$ Relocation!H27*P27 & $=\$ C \$ 7^{*} \$ D 27^{*}$ Relocation!E27 $\$ C \$ 35 /$ SE $\$ 35^{*} P 27$ \\
\hline 28 & Pu pure-242 & $=\$ C \$ 7^{*} \$ C \$ 3^{*} \$ C \$ 4^{*} \$ C \$ 5^{*} \$ C \$ 6^{*} F 28^{*}$ Relocation!H28*P28 & $=\$ C \$ 7^{*} \$ D 28^{*}$ Relocation!E28*\$C $\$ 35 / \$ E \$ 35^{*} \mathrm{P} 28$ \\
\hline 29 & Sr-90 & $=\$ C \$ 7^{*} \$ C \$ 3^{*} \$ C \$ 4^{*} \$ C \$ 5^{*} \$ C \$ 6^{*} F 29^{*}$ Relocation!H29*P29 & $=\$ C \$ 7^{*} \$ D 29^{*}$ Relocation!E29*\$C\$35/\$E $\$ 35^{*} P 29$ \\
\hline 30 & Ta-99 & $=\$ C \$ 7^{*} \$ C \$ 3^{*} \$ C \$ 4^{*} \$ C \$ 5^{*} \$ C \$ 6^{*} F 30^{*}$ Relocation!H30*P30 & $=\$ C \$ 7 * \$ D 30 *$ Relocation!E30*\$C\$35/\$E $\$ 35 * P 30$ \\
\hline 31 & Th-232 & $=\$ C \$ 7^{*} \$ C \$ 3^{*} \$ C \$ 4^{*} \$ C \$ 5^{*} \$ C \$ 6^{*} F 31^{*}$ Relocation!H31*P31 & $=\$ C \$ 7^{*} \$ D 31^{*}$ Relocation!E31*\$C\$35/\$E $\$ 35^{*} P 31$ \\
\hline 32 & TI-204 & $=\$ C \$ 7^{*} \$ C \$ 3^{*} \$ C \$ 4^{*} \$ C \$ 5^{*} \$ C \$ 6^{*} F 32^{*}$ Relocation!H32*P32 & $=\$ C \$ 7^{*} \$ D 32^{*}$ Relocation!E32*\$C\$35/\$E $\$ 35^{*} P 32$ \\
\hline 33 & U-235 & $=\$ C \$ 7^{*} \$ C \$ 3^{*} \$ C \$ 4^{*} \$ C \$ 5^{*} \$ C \$ 6^{*} F 33^{*}$ Relocation $1 H 33^{*} P 33$ & $=\$ C \$ 7^{*} \$ D 33^{*}$ Relocation!E33*\$C\$35/\$E $\$ 35^{*} \mathrm{P} 33$ \\
\hline 34 & U-238 & $=\$ C \$ 7^{*} \$ C \$ 3^{*} \$ C \$ 4^{*} \$ C \$ 5^{*} \$ C \$ 6^{*} F 34^{*}$ Relocation!H34*P34 & $=\$ C \$ 7^{*} \$ D 34^{*}$ Relocation!E34*\$C\$35/\$E $\$ 35^{*} P 34$ \\
\hline 35 & Total & & \\
\hline 36 & Ba-137m & & $=\$ C \$ 7^{*} \$ D 36^{*}$ Relocation!E36*\$C $\$ 35 / \$ E \$ 35^{*} \mathrm{P} 36$ \\
\hline 37 & Y-90 & & $=\$ C \$ 7^{*} \$ D 37^{*}$ Relocation!E37*\$C $\$ 35 / \$ E \$ 35^{* P 37}$ \\
\hline 38 & Pr-144 & & $=\$ C \$ 7 * \$ D 38^{*}$ Relocation!E38*\$C $\$ 35 / \$ E \$ 35^{*} \mathrm{P} 38$ \\
\hline 39 & $\mathrm{~Pa}-233$ & & $=\$ C \$ 7^{*} \$ D 39^{*}$ Relocation!E39*\$C\$35/\$E $\$ 35^{*} P 39$ \\
\hline 40 & Th-234 & & $=\$ C \$ 7^{*} \$ D 40^{*}$ Relocation!E $40^{*} \$ C \$ 35 / \$ E \$ 35^{*} P 40$ \\
\hline 41 & $\mathrm{~Pa}-234$ & & $=\$ C \$ 7^{*} \$ D 41^{*}$ Relocation!E $41^{*} \$ C \$ 35 / \$ E \$ 35^{*} P 41$ \\
\hline 42 & Np-239 & & $=\$ C \$ 7^{*} \$ D 42^{*}$ Relocation!E42*\$C $\$ 35 / \$ E \$ 35^{*} \mathrm{P} 42$ \\
\hline 43 & Th-231 & & $=\$ C \$ 7^{*} \$ D 43^{*}$ Relocation!E $43^{*} \$ C \$ 35 / \$ E \$ 35^{*}$ P43 \\
\hline 44 & Ra-228 & & $=\$ C \$ 7^{*} \$ D 44^{*}$ Relocation!E44*\$C $\$ 35 / \$ E \$ 35^{*}$ P44 \\
\hline 45 & Ac-228 & & $=\$ C \$ 7 * \$ D 45^{*}$ Relocation!E $45^{*} \$ C \$ 35 / \$ E \$ 35^{*} \mathrm{P} 45$ \\
\hline 46 & & & \\
\hline 47 & Total & $=\mathrm{SUM}(\mathrm{H} 10: \mathrm{H} 46)$ & $=S U M(110: 146)$ \\
\hline 48 & & & \\
\hline 49 & \multicolumn{2}{|c|}{ Dose in seco:H47-'Relo_SRTC 1yr!H47 } & $=147-$ Relo_SRTC 1yr'!147 \\
\hline
\end{tabular}




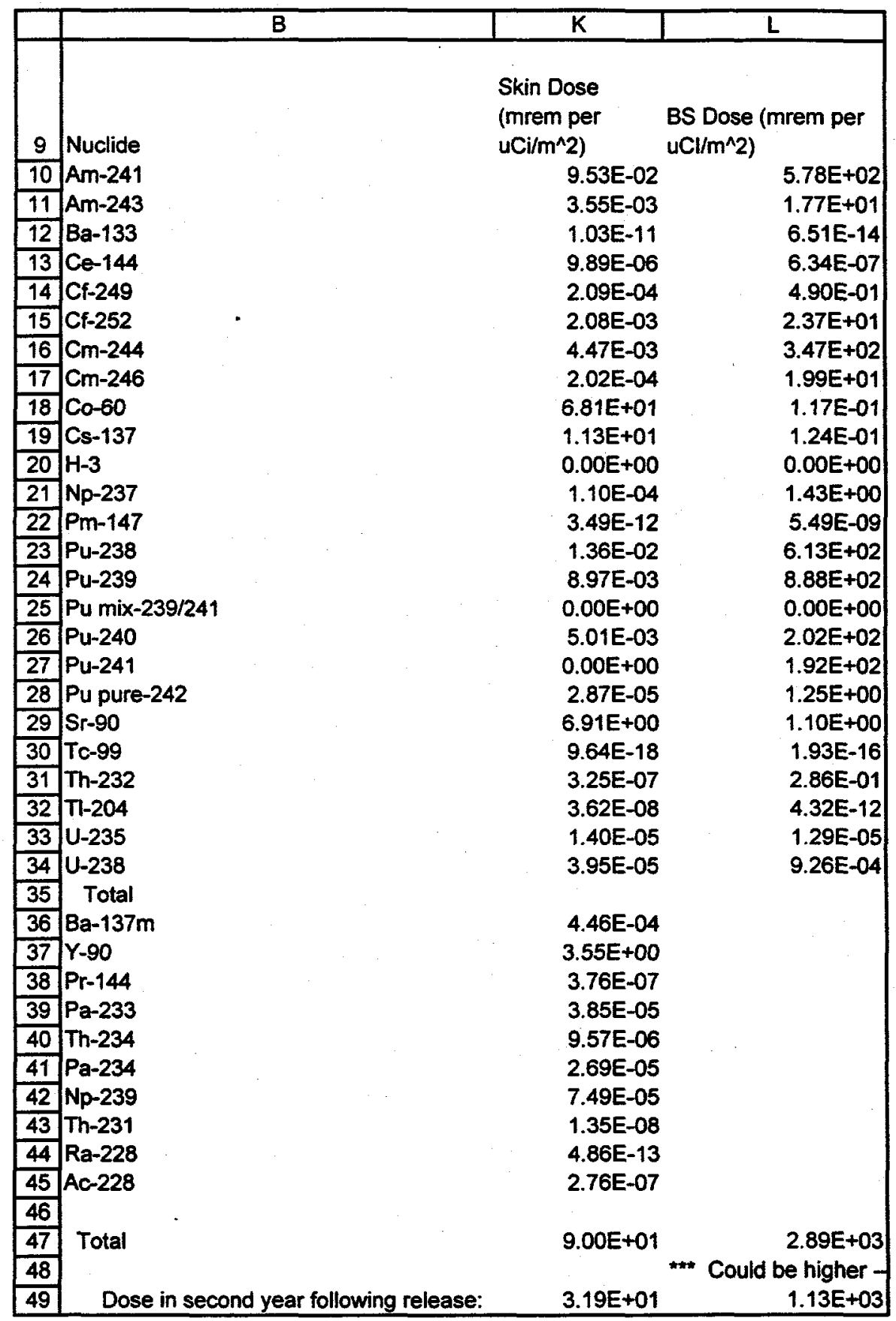




\begin{tabular}{|c|c|c|c|}
\hline & $\mathrm{B}$ & $\bar{K}$ & $\underline{L}$ \\
\hline 9 & Nuclide & Skin Dose (mrem per $u \mathrm{Ci}^{\prime} / \mathrm{m}^{\wedge} 2$ ) & BS Dose (mrem per $\left.u C l / m^{\wedge} 2\right)$ \\
\hline 10 & Am-241 & $=\$ C \$ 7^{*} \$ D 10^{*}$ Relocation! $\$ F 10^{*} \$ C \$ 35 / \$ E \$ 35^{*} P 10$ & $=\$ C \$ 7^{*} \$ C \$ 3^{*} \$ C \$ 4^{*} \$ C \$ 5^{*} \$ C \$ 6^{*} \$ F 10^{*}$ Relocation $\$ J 10^{*} \mathrm{P} 10$ \\
\hline 11 & Am-243 & $=\$ C \$ 7^{*} \$ D 11 *$ Relocation! $\$ F 11 * \$ C \$ 35 / \$ E \$ 35^{*} P 11$ & $=\$ C \$ 7^{*} \$ C \$ 3^{*} \$ C \$ 4^{*} \$ C \$ 5^{*} \$ C \$ 6^{*} \$ F 11^{*}$ Relocation! $\$ 111^{* P 11}$ \\
\hline 12 & Ba-133 & $=\$ C \$ 7^{*} \$ D 12^{*}$ Relocation! $\$ F 12^{*} \$ C \$ 35 / \$ E \$ 35^{*} P 12$ & $=\$ C \$ 7^{*} \$ C \$ 3^{*} \$ C \$ 4^{*} \$ C \$ 5^{*} \$ C \$ 6^{*} \$ F 12^{*}$ Relocation! $\$ J 12^{*} \mathrm{P} 12$ \\
\hline 13 & Ce-144 & $=\$ C \$ 7^{*} \$ D 13^{*}$ Relocation!\$F13*\$C\$35/\$E\$35*P13 & $=\$ C \$ 7^{*} \$ C \$ 3^{*} \$ C \$ 4^{*} \$ C \$ 5^{*} \$ C \$ 6^{*} \$ F 13^{*}$ Relocation! $\$ 13^{*} P 13$ \\
\hline 14 & CF-249 & $=\$ C \$ 7^{*} \$ D 14^{*}$ Relocation!\$F14*\$C\$35/\$E\$35*P14 & $=\$ C \$ 7^{*} \$ C \$ 3^{*} \$ C \$ 4^{*} \$ C \$ 5^{*} \$ C \$ 6^{*} \$ F 14^{*}$ Relocation! $\$ 14^{*} \mathrm{P} 14$ \\
\hline 15 & Cf-252 & $=\$ C \$ 7^{*} \$ D 15^{*}$ Relocation!\$F $15^{*} \$ C \$ 35 / \$ E \$ 35^{\star} P 15$ & $=\$ C \$ 7^{*} \$ C \$ 3^{*} \$ C \$ 4^{*} \$ C \$ 5^{*} \$ C \$ 6^{*} \$ F 15^{*}$ Relocation!\$J15*P15 \\
\hline 16 & $\mathrm{Cm}-244$ & $=\$ C \$ 7^{*} \$ D 16^{*}$ Relocation!\$F16*\$C $\$ 35 / \$ E \$ 35^{*}$ P 16 & $=\$ C \$ 7^{*} \$ C \$ 3^{*} \$ C \$ 4^{*} \$ C \$ 5^{*} \$ C \$ 6^{*} \$ F 16^{*}$ Relocation!\$J16*P16 \\
\hline 17 & $\mathrm{Cm}-246$ & $=\$ C \$ 7^{*} \$ D 17^{*}$ Relocation!\$F17*\$C\$35/\$E\$35*P17 & $=\$ C \$ 7^{*} \$ C \$ 3^{*} \$ C \$ 4^{*} \$ C \$ 5^{*} \$ C \$ 6^{*} \$ F 17^{*}$ Relocation $1 \$ 17^{*} P 17$ \\
\hline 18 & Co-60 & $=\$ C \$ 7^{*} \$ D 18^{*}$ Relocation!\$F18*\$C\$35/\$E\$35*P18 & $=\$ C \$ 7^{*} \$ C \$ 3^{*} \$ C \$ 4^{*} \$ C \$ 5^{*} \$ C \$ 6^{*} \$ F 18^{*}$ Relocation $\$ N 18^{*} P 18$ \\
\hline 19 & Cs-137 & $=\$ C \$ 7^{*} \$ D 19^{*}$ Relocation!\$F $19 * \$ C \$ 35 / \$ E \$ 35^{*} \mathrm{P} 19$ & $=\$ C \$ 7^{*} \$ C \$ 3^{*} \$ C \$ 4^{*} \$ C \$ 5^{*} \$ C \$ 6^{*} \$ F 19^{*}$ Relocation!\$J19*P19 \\
\hline 20 & $\mathrm{H}-3$ & $=\$ C \$ 7^{*} \$ D 20^{*}$ Relocation! $\$ F 20^{*} \$ C \$ 35 / \$ E \$ 35^{*} P 20$ & $=\$ C \$ 7^{*} \$ C \$ 3^{*} \$ C \$ 4^{*} \$ C \$ 5^{*} \$ C \$ 6^{*} \$ F 20^{*}$ Relocation $1 \$ 22^{*} \mathrm{P} 20$ \\
\hline 21 & No-237 & $=\$ C \$ 7^{*} \$ D 21^{*}$ Relocation!\$F21*\$C\$35/\$E\$35*P21 & $=\$ C \$ 7^{*} \$ C \$ 3^{*} \$ C \$ 4^{*} \$ C \$ 5^{*} \$ C \$ 6^{*} \$ F 21^{*}$ Relocation! $\$ 22^{*} P 21$ \\
\hline 22 & $\mathrm{Pm}-147$ & $=\$ C \$ 7^{*} \$ D 22^{*}$ Relocation! $\$ 22^{*} \$ C \$ 35 / \$ E \$ 35^{*} P 22$ & $=\$ C \$ 7^{*} \$ C \$ 3^{*} \$ C \$ 4^{*} \$ C \$ 5^{*} \$ C \$ 6^{*} \$ F 22^{*}$ Relocation! $\$ 122^{*} \mathrm{P} 22$ \\
\hline 23 & Pu-238 & $=\$ C \$ 7^{*} \$ D 23^{*}$ Relocation! $\$ F 23^{*} \$ C \$ 35 / \$ E \$ 35^{*} P 23$ & $=\$ C \$ 7^{*} \$ C \$ 3^{*} \$ C \$ 4^{*} \$ C \$ 5^{*} \$ C \$ 6^{*} \$ F 23^{*}$ Relocation!\$J23*P23 \\
\hline 24 & Pu-239 & $=\$ C \$ 7^{*} \$ D 24^{*}$ Relocation!\$F24*\$C\$35/\$E $\$ 35^{*}$ P24 & $=\$ C \$ 7^{*} \$ C \$ 3^{*} \$ C \$ 4^{*} \$ C \$ 5^{*} \$ C \$ 6^{*} \$ F 24^{*}$ Relocation! $\$ 24^{*} \mathrm{P} 24$ \\
\hline 25 & Pu mix-239/24 & $4=\$ C \$ 7^{*} \$ D 25^{*}$ Relocation $\$ F 25 * \$ C \$ 35 / \$ E \$ 35^{*}$ P25 & $=\$ C \$ 7^{*} \$ C \$ 3^{*} \$ C \$ 4^{*} \$ C \$ 5^{*} \$ C \$ 6^{*} \$ F 25^{*}$ Relocation $\mid \$ J 25^{*} P 25$ \\
\hline 26 & Pu-240 & $=\$ C \$ 7^{*} \$ D 26^{*}$ Relocation $\$$ \$F $26^{*} \$ C \$ 35 / \$ E \$ 35^{*} P 26$ & $=\$ C \$ 7^{*} \$ C \$ 3^{*} \$ C \$ 4^{*} \$ C \$ 5^{*} \$ C \$ 6^{*} \$ F 26^{*}$ Relocation $! \$ 26^{*} P 26$ \\
\hline 27 & Pu-241 & $=\$ C \$ 7^{*} \$ D 27^{*}$ Relocation! $\$ F 27^{*} \$ C \$ 35 / \$ E \$ 35^{*} P 27$ & $=\$ C \$ 7^{*} \$ C \$ 3^{*} \$ C \$ 4^{*} \$ C \$ 5^{*} \$ C \$ 6^{*} \$ 27^{*}$ Relocation $\mid \$ 27^{*} \mathrm{P} 27$ \\
\hline 28 & Pu pure-242 & $=\$ C \$ 7^{*} \$ D 28^{*}$ Relocation!\$F28*\$C $\$ 35 / \$ E \$ 35^{*} P 28$ & $=\$ C \$ 7^{*} \$ C \$ 3^{*} \$ C \$ 4^{*} \$ C \$ 5^{*} \$ C \$ 6^{*} \$ F 28^{*}$ Relocation $\$ J 28^{*} P 28$ \\
\hline 29 & Sr-90 & $=\$ C \$ 7^{*} \$ D 29^{*}$ Relocation $\$ F 29^{*} \$ C \$ 35 / \$ E \$ 35^{*} P 29$ & $=\$ C \$ 7^{*} \$ C \$ 3^{*} \$ C \$ 4^{*} \$ C \$ 5^{*} \$ C \$ 6^{*} \$ F 29^{*}$ Relocation! $\$ J 29^{*} P 29$ \\
\hline 30 & |Tc-99 & $=\$ C \$ 7^{*} \$ D 30^{*}$ Relocation $1 \$ F 30^{*} \$ C \$ 35 / \$ E \$ 35^{*} \mathrm{P} 30$ & $=\$ C \$ 7^{*} \$ C \$ 3^{*} \$ C \$ 4^{*} \$ C \$ 5^{*} \$ C \$ 6^{*} \$ F 30^{*}$ Relocation $\mid \$ 30^{*} \mathrm{P} 30$ \\
\hline 31 & Th-232 & $=\$ C \$ 7 * \$ D 31 *$ Relocation $\$$ \$F $31 * \$ C \$ 35 / \$ E \$ 35 * P 31$ & $=\$ C \$ 7^{*} \$ C \$ 3^{*} \$ C \$ 4^{*} \$ C \$ 5^{*} \$ C \$ 6^{*} \$ F 31^{*}$ Relocation!\$131*P31 \\
\hline 32 & $\mathrm{TI}-204$ & $=\$ C \$ 7^{*} \$ D 32^{*}$ Relocation! $\$ F 32^{*} \$ C \$ 35 / \$ E \$ 35^{*} P 32$ & $=\$ C \$ 7^{*} \$ C \$ 3^{*} \$ C \$ 4^{*} \$ C \$ 5^{*} \$ C \$ 6^{*} \$ F 32^{*}$ Relocation! $\$ J 32^{*} P 32$ \\
\hline 33 & U-235 & $=\$ C \$ 7^{*} \$ D 33^{*}$ Relocation!\$F33*\$C\$35/\$E $\$ 35^{*} \mathrm{P} 33$ & $=\$ C \$ 7^{*} \$ C \$ 3^{*} \$ C \$ 4^{*} \$ C \$ 5^{*} \$ C \$ 6^{*} \$ F 33^{*}$ Relocation $\mid \$ J 33^{*} P 33$ \\
\hline 34 & U-238 & $=\$ C \$ 7^{*} \$ D 34^{*}$ Relocation $\$$ \$F $34^{*} \$ C \$ 35 / \$ E \$ 35^{*} P 34$ & $=\$ C \$ 7^{*} \$ C \$ 3^{*} \$ C \$ 4^{*} \$ C \$ 5^{*} \$ C \$ 6^{*} \$ F 34^{*}$ Relocation! $\$ J 34^{*} P 34$ \\
\hline 35 & Total & & \\
\hline 36 & Ba-137m & $=\$ C \$ 7^{*} \$ D 36^{*}$ Relocation!\$F36*\$C $\$ 35 / \$ E \$ 35^{*} P 36$ & \\
\hline 37 & $\mathrm{Y}-90$ & $=\$ C \$ 7^{*} \$ D 37^{*}$ Relocation!\$F37*\$C\$35/\$E\$35*P37 & \\
\hline 38 & Pr-144 & $=\$ C \$ 7^{*} \$ D 38^{*}$ Relocation! $\$ F 38^{*} \$ C \$ 35 / \$ E \$ 35^{*} P 38$ & \\
\hline 39 & Pa-233 & $=\$ C \$ 7^{*} \$ D 39^{*}$ Relocation $1 \$ F 39^{*} \$ C \$ 35 / \$ E \$ 35^{*} P 39$ & · \\
\hline 40 & Th-234 & $=\$ C \$ 7^{*} \$ D 40^{*}$ Relocation $\$ F 40^{*} \$ C \$ 35 / \$ E \$ 35^{*} P 40$ & \\
\hline 41 & $\mathrm{~Pa}-234$ & $=\$ C \$ 7^{*} \$ D 41^{*}$ Relocation $1 \$ 44^{*} \$ C \$ 35 / \$ E \$ 35^{*} P 41$ & \\
\hline 42 & Np-239 & $=\$ C \$ 7^{*} \$ D 42^{*}$ Relocation!\$F $42^{*} \$ C \$ 35 / \$ E \$ 35^{*} P 42$ & \\
\hline 43 & Th-231 & $=\$ C \$ 7^{*} \$ D 43^{*}$ Relocation!\$F $43^{*} \$ C \$ 35 / \$ E \$ 35^{*} \mathrm{P} 43$ & \\
\hline 44 & Ra-228 & $=\$ C \$ 7^{*} \$ D 44^{*}$ Relocation!\$F44*\$C $\$ 35 / \$ E \$ 35^{*} P 44$ & \\
\hline 45 & Ac-228 & $=\$ C \$ 7^{*} \$ D 45^{*}$ Relocation $1 \$ F 45^{*} \$ C \$ 35 / \$ E \$ 35^{*} P 45$ & \\
\hline 46 & & & \\
\hline 47 & Total & $=$ SUM(K10:K46) & $=S U M(L 10: L 46)$ \\
\hline 48 & & & *** Could be higher - DCFs were taken based on limiti \\
\hline 49 & Dose in se & $=K 47-'$ Relo_SRTC 1yr!K47 & $=$ L47-'Relo_SRTC 1yr!L47 \\
\hline
\end{tabular}




\begin{tabular}{|c|c|c|c|}
\hline & $\bar{B}$ & $M$ & $\bar{N}$ \\
\hline 9 & Nuclide & Halflife (years) & Lambda1 \\
\hline 10 & Am-241 & 458 & 0.0015131 \\
\hline 11 & $A m-243$ & 7950 & $8.71698 E-05$ \\
\hline 12 & $B a-133$ & 10.5 & 0.066 \\
\hline 13 & $C e-144$ & 0.778082192 & 0.890651408 \\
\hline 14 & $C f-249$ & 360 & 0.001925 \\
\hline 15 & Cf-252 & 2.646 & 0.261904762 \\
\hline 16 & $\mathrm{Cm}-244$ & 17.6 & 0.039375 \\
\hline 17 & $\mathrm{Cm}-246$ & 5500 & 0.000126 \\
\hline 18 & Co-60 & 5.26 & 0.131749049 \\
\hline 19 & Cs-137 & 30 & 0.0231 \\
\hline 20 & $\mathrm{H}-3$ & 12.3 & 0.056341463 \\
\hline 21 & $\mathrm{~Np}-237$ & 2140000 & $3.23832 E-07$ \\
\hline 22 & Pm-147 & 2.62 & 0.264503817 \\
\hline 23 & Pu-238 & 86.4 & 0.008020833 \\
\hline 24 & Pu-239 & 24390 & $2.84133 E-05$ \\
\hline 25 & Pu mix-239/241 & & \\
\hline 26 & Pu-240 & 6580 & 0.000105319 \\
\hline 27 & Pu-241 & 13.2 & 0.0525 \\
\hline 28 & Pu pure-242 & 379000 & $1.8285 E-06$ \\
\hline 29 & Sr-90 & 28.1 & 0.024661922 \\
\hline 30 & TC-99 & 212000 & $3.26887 E-06$ \\
\hline 31 & Th-232 & 14000000000 & 4.95E-11 \\
\hline 32 & $T 1-204$ & 3.8 & 0.182368421 \\
\hline 33 & U-235 & 710000000 & $9.76056 \mathrm{E}-10$ \\
\hline 34 & U-238 & 4510000000 & 1.53659E-10 \\
\hline 35 & Total & & \\
\hline 36 & $\mathrm{Ba}-137 \mathrm{~m}$ & 4.8554E-06 & 142727.5862 \\
\hline 37 & $Y-90$ & 0.007317352 & 94.70639626 \\
\hline 38 & Pr-144 & 3.28767E-05 & 21078.75 \\
\hline 39 & Pa-233 & 0.073972603 & 9.368333333 \\
\hline 40 & Th-234 & 0.066027397 & 10.49564315 \\
\hline 41 & $\mathrm{~Pa}-234$ & 0.00076484 & 906.0716418 \\
\hline 42 & $N p-239$ & 0.006452055 & 107.4076433 \\
\hline 43 & Th-231 & 0.002913242 & 237.8793103 \\
\hline 44 & $\operatorname{Ra}-228$ & 5.75 & 0.120521739 \\
\hline 45 & $A c-228$ & 0.000699772 & 990.3230016 \\
\hline
\end{tabular}




\begin{tabular}{|c|c|c|c|c|c|}
\hline & B & $\bar{M}$ & & $\mathbf{N}$ & \\
\hline 9 & Nuclide & Halfifife (years) & Lambda1 & & \\
\hline 10 & Am-241 & $=T$ half!C2 & $=0.693 / \mathrm{M} 10$ & & \\
\hline 11 & Am-243 & $=T$ half!C3 & $=0.693 / \mathrm{M} 11$ & & \\
\hline 12 & $\mathrm{Ba}-133$ & $=T$ halflC4 & $=0.693 / \mathrm{M} 12$ & & \\
\hline 13 & Ce-144 & $=T_{-}$halfiC5 & $=0.693 / \mathrm{M} 13$ & & - \\
\hline 14 & Cf-249 & $=T_{-}$half!C6 & $=0.693 / M_{14}$ & & \\
\hline 15 & Cf-252 & $=T^{-}$halfic7 & $=0.693 / \mathrm{M} 15$ & & \\
\hline 16 & $\mathrm{Cm}-244$ & $=T \_$half!C8 & $=0.693 / \mathrm{M} 16$ & & \\
\hline 17 & $\mathrm{Cm}-246$ & =T_half!C9 & $=0.693 / \mathrm{M} 17$ & & \\
\hline 18 & Co-60 & $=T_{-}$half $1 \mathrm{C} 10$ & $=0.693 / \mathrm{M} 18$ & & \\
\hline 19 & Cs-137 & $=T$ half!C11 & $=0.693 / \mathrm{M} 19$ & & \\
\hline 20 & $H-3$ & $=T_{\text {_half! }}$ C12 & $=0.693 / \mathrm{M} 20$ & . & \\
\hline 21 & Np-237 & $=T_{\text {_halfl }} \mathrm{C} 13$ & $=0.693 / \mathrm{M} 21$ & & \\
\hline 22 & Pm-147 & $=T$ half!C14 & $=0.693 / \mathrm{M} 22$ & & \\
\hline 23 & Pu-238 & $=T_{\text {_halfl }} \mathrm{C} 15$ & $=0.693 / \mathrm{M} 23$ & & \\
\hline 24 & $P u-239$ & $=T$ _half!C16 & $=0.693 / \mathrm{M} 24$ & & \\
\hline 25 & Pu mix-239/241 & & & & \\
\hline 26 & Pu-240 & $=T_{\text {_half }} \mathrm{C} 18$ & $=0.693 / \mathrm{M} 26$ & & \\
\hline 27 & Pu-241 & $=T$ halfic19 & $=0.693 / \mathrm{M} 27$ & & \\
\hline 28 & Pu pure-242 & $=T$ half $! C 20$ & $=0.693 / \mathrm{M} 28$ & & \\
\hline 29 & Sr-90 & $=T$ half!C21 & $=0.693 / \mathrm{M} 29$ & & \\
\hline 30 & Tc-99 & $=T_{\text {_half }} \mathrm{C} 22$ & $=0.693 / \mathrm{M} 30$ & & \\
\hline 31 & Th-232 & $=T$ _half!C23 & $=0.693 / \mathrm{M} 31$ & & \\
\hline 32 & TI-204 & $=T$ Thalf!C24 & $=0.693 / \mathrm{M} 32$ & & \\
\hline 33 & $U-235$ & $=T_{\text {_half }} \mathrm{C} 25$ & $=0.693 / \mathrm{M} 33$ & & \\
\hline 34 & U-238 & $=T$ _half!C26 & $=0.693 / \mathrm{M} 34$ & & \\
\hline 35 & Total & & & & \\
\hline 36 & Ba-137m & $=T$ _half!C28 & $=0.693 / \mathrm{M} 36$ & & \\
\hline 37 & $Y-90$ & $=T_{\text {__half }}$ C29 & $=0.693 / \mathrm{M} 37$ & & \\
\hline 38 & Pr-144 & $=T$ _half!C30 & $=0.693 / \mathrm{M} 38$ & & \\
\hline 39 & Pa-233 & $=T$ _half!C31 & $=0.693 / \mathrm{M} 39$ & & \\
\hline 40 & Th-234 & $=T$ half!C32 & $=0.693 / \mathrm{M} 40$ & & \\
\hline 41 & Pa-234 & $=T$ _half!C33 & $=0.693 / \mathrm{M} 41$ & & \\
\hline 42 & Np-239 & $=T_{\text {T_half!C } 34}$ & $=0.693 / \mathrm{M} 42$ & & \\
\hline 43 & Th-231 & $=T$-half!C35 & $=0.693 / \mathrm{M} 43$ & & \\
\hline 44 & Ra-228 & $=T$ _half!C36 & $=0.693 / \mathrm{M} 44$ & & \\
\hline 45 & AC-228 & $=T^{-}$half!C37 & $=0.693 / \mathrm{M} 45$ & & \\
\hline
\end{tabular}




\begin{tabular}{|c|c|c|}
\hline & $\bar{B}$ & $P$ \\
\hline 9 & Nuclide & $\begin{array}{l}\text { Weathering } \\
\text { Factor ( } 2 \text { years) }\end{array}$ \\
\hline 10 & $A m-241$ & $1.232 E+00$ \\
\hline 11 & Am-243 & $1.234 E+00$ \\
\hline 12 & $\mathrm{Ba}-133$ & $1.167 \mathrm{E}+00$ \\
\hline 13 & Ce-144 & - $\quad 6.499 E-01$ \\
\hline 14 & Cf-249 & $1.232 E+00$ \\
\hline 15 & Cf-252 & 9.968E-01 \\
\hline 16 & $\mathrm{Cm}-244$ & 1.193E+00 \\
\hline 17 & $\mathrm{Cm}-246$ & $1.234 E+00$ \\
\hline 18 & Co-60 & $1.105 E+00$ \\
\hline 19 & Cs-137 & $1.210 E+00$ \\
\hline 20 & $H-3$ & $1.176 \mathrm{E}+00$ \\
\hline 21 & $\mathrm{~Np}-237$ & $1.234 E+00$ \\
\hline 22 & $\mathrm{Pm}-147$ & 9.948E-01 \\
\hline 23 & Pu-238 & $1.225 E+00$ \\
\hline 24 & $P u-239$ & $1.234 E+00$ \\
\hline 25 & Pu mix-239/241 & \\
\hline 26 & Pu-240 & $1.234 E+00$ \\
\hline 27 & $P u-241$ & 1.180E+O0 \\
\hline 28 & Pu pure-242 & $1.234 E+00$ \\
\hline 29 & Sr-90 & $1.208 \mathrm{E}+00$ \\
\hline 30 & TC-99 & $1.234 E+00$ \\
\hline 31 & Th-232 & $1.234 E+00$ \\
\hline 32 & TI-204 & $1.061 E+00$ \\
\hline 33 & $\mathrm{U}-235$ & $1.234 E+00$ \\
\hline 34 & U-238 & $1.234 E+00$ \\
\hline 35 & Total & \\
\hline 36 & $\mathrm{Ba}-137 \mathrm{~m}$ & 7.006E-06 \\
\hline 37 & $Y-90$ & 1.048E-02 \\
\hline 38 & Pr-144 & 4.744E-05 \\
\hline 39 & $\mathrm{~Pa}-233$ & 9.947E-02 \\
\hline 40 & Th-234 & $8.942 E-02$ \\
\hline 41 & Pa-234 & 1.103E-03 \\
\hline 42 & $N p-239$ & $9.249 \mathrm{E}-03$ \\
\hline 43 & Th-231 & 4.191E-03 \\
\hline 44 & Ra-228 & $1.115 E+00$ \\
\hline 45 & Ac-228 & $1.009 E-03$ \\
\hline
\end{tabular}




\begin{tabular}{|c|c|c|}
\hline & $\bar{B}$ & $P$ \\
\hline 9 & Nuclide & Weathering Factor ( 2 years) \\
\hline 10 & Am-241 & $=\left(0.63 /(\$ N 10+\$ L \$ 4)^{*}\left(1-\operatorname{EXP}\left(-(\$ N 10+\$ L \$ 4)^{*} \$ O \$ 5\right)\right)\right)+\left((0.37 /(\$ N 10+\$ L \$ 6))^{*}\left(1-\operatorname{EXP}\left(-(\$ N 10+\$ L \$ 6)^{*} \$ 0 \$ 5\right)\right)\right)$ \\
\hline 11 & Am-243 & $=\left(0.63 /(\$ N 11+\$ L \$ 4)^{*}\left(1-\operatorname{EXP}\left(-(\$ N 11+\$ L \$ 4)^{*} \$ O \$ 5\right)\right)\right)+\left((0.37 /(\$ N 11+\$ L \$ 6))^{*}\left(1-\operatorname{EXP}\left(-(\$ N 11+\$ L \$ 6)^{*} \$ O \$ 5\right)\right)\right)$ \\
\hline 12 & Ba-133 & $=\left(0.63 /(\$ N 12+\$ L \$ 4)^{*}\left(1-\operatorname{EXP}\left(-(\$ N 12+\$ L \$ 4)^{*} \$ O \$ 5\right)\right)\right)+\left((0.37 /(\$ N-12+\$ L \$ 6))^{*}\left(1-\operatorname{EXP}\left(-(\$ N 12+\$ L \$ 6)^{*} \$ O \$ 5\right)\right)\right)$ \\
\hline 13 & $\mathrm{Ce}-144$ & $=\left(0.63 /(\$ N 13+\$ L \$ 4)^{*}\left(1-\operatorname{EXP}\left(-(\$ N 13+\$ L \$ 4)^{*} \$ O \$ 5\right)\right)\right)+\left((0.37 /(\$ N 13+\$ L \$ 6))^{*}\left(1-\operatorname{EXP}\left(-(\$ N 13+\$ L \$ 6)^{*} \$ O \$ 5\right)\right)\right)$ \\
\hline 14 & Cf-249 & $=\left(0.63 /(\$ N 14+\$ L \$ 4)^{*}\left(1-\operatorname{EXP}\left(-(\$ N 14+\$ L \$ 4)^{*} \$ O \$ 5\right)\right)\right)+\left((0.37 /(\$ N 14+\$ L \$ 6))^{*}\left(1-\operatorname{EXP}\left(-(\$ N 14+\$ L \$ 6)^{*} \$ O \$ 5\right)\right)\right)$ \\
\hline 15 & Cf-252 & $=\left(0.63 /(\$ N 15+\$ L \$ 4)^{*}\left(1-\operatorname{EXP}\left(-(\$ N 15+\$ L \$ 4)^{*} \$ O \$ 5\right)\right)\right)+\left((0.37 /(\$ N 15+\$ L \$ 6))^{*}\left(1-\operatorname{EXP}\left(-(\$ N 15+\$ L \$ 6)^{*} \$ O \$ 5\right)\right)\right)$ \\
\hline 16 & $\mathrm{Cm}-244$ & $=\left(0.63 /(\$ N 16+\$ L \$ 4)^{*}\left(1-\operatorname{EXP}\left(-(\$ N 16+\$ L \$ 4)^{*} \$ O \$ 5\right)\right)\right)+\left((0.37 /(\$ N 16+\$ L \$ 6))^{*}\left(1-\operatorname{EXP}\left(-(\$ N 16+\$ L \$ 6)^{*} \$ O 55\right)\right)\right)$ \\
\hline 17 & $\mathrm{Cm}-246$ & $=\left(0.63 /(\$ N 17+\$ L \$ 4)^{*}\left(1-\operatorname{EXP}\left(-(\$ N 17+\$ L \$ 4)^{*} \$ O \$ 5\right)\right)\right)+\left((0.37 /(\$ N 17+\$ L \$ 6))^{*}\left(1-\operatorname{EXP}\left(-(\$ N 17+\$ L \$ 6)^{*} \$ O \$ 5\right)\right)\right)$ \\
\hline 18 & Co-60 & $=\left(0.63 /(\$ N 18+\$ L \$ 4)^{*}\left(1-\operatorname{EXP}\left(-(\$ N 18+\$ L \$ 4)^{*} \$ O \$ 5\right)\right)\right)+\left((0.37 /(\$ N 18+\$ L \$ 6))^{*}\left(1-\operatorname{EXP}\left(-(\$ N 18+\$ L \$ 6)^{*} \$ O 55\right)\right)\right)$ \\
\hline 19 & Cs-137 & $=\left(0.63 /(\$ N 19+\$ L \$ 4)^{*}\left(1-\operatorname{EXP}\left(-(\$ N 19+\$ L \$ 4)^{*} \$ 0 \$ 5\right)\right)\right)+\left((0.37 /(\$ N 19+\$ L \$ 6))^{*}\left(1-\operatorname{EXP}\left(-(\$ N 19+\$ L \$ 6)^{*} \$ O \$ 5\right)\right)\right)$ \\
\hline 20 & $\mathrm{H}-3$ & $=\left(0.63 /(\$ N 20+\$ L \$ 4)^{*}\left(1-\operatorname{EXP}\left(-(\$ N 20+\$ L \$ 4)^{*} \$ O \$ 5\right)\right)\right)+\left((0.37 /(\$ N 20+\$ L \$ 6))^{*}\left(1-\operatorname{EXP}\left(-(\$ N 20+\$ L \$ 6)^{*} \$ O \$ 5\right)\right)\right)$ \\
\hline 21 & Np-237 & $=\left(0.63 /(\$ N 21+\$ L \$ 4)^{*}\left(1-\operatorname{EXP}\left(-(\$ N 21+\$ L \$ 4)^{*} \$ O \$ 5\right)\right)\right)+\left((0.37 /(\$ N 21+\$ L \$ 6))^{*}\left(1-\operatorname{EXP}\left(-(\$ N 21+\$ L \$ 6)^{*} \$ O \$ 5\right)\right)\right)$ \\
\hline 22 & $\mathrm{Pm}-147$ & $=\left(0.63 /(\$ N 22+\$ L \$ 4)^{*}\left(1-\operatorname{EXP}\left(-(\$ N 22+\$ L \$ 4)^{*} \$ O \$ 5\right)\right)\right)+\left((0.37 /(\$ N 22+\$ L \$ 6))^{*}\left(1-\operatorname{EXP}\left(-(\$ N 22+\$ L \$ 6)^{*} \$ O \$ 5\right)\right)\right)$ \\
\hline 23 & Pu-238 & $=\left(0.63 /(\$ N 23+\$ L \$ 4)^{*}\left(1-\operatorname{EXP}\left(-(\$ N 23+\$ L \$ 4)^{*} \$ O \$ 5\right)\right)\right)+\left((0.37 /(\$ N 23+\$ L \$ 6))^{*}\left(1-\operatorname{EXP}\left(-(\$ N 23+\$ L \$ 6)^{*} \$ O \$ 5\right)\right)\right)$ \\
\hline$\frac{24}{25}$ & $\begin{array}{l}\text { Pu-239 } \\
\text { Pu mix-239/241 }\end{array}$ & $=\left(0.63 /(\$ N 24+\$ L \$ 4)^{*}(1-\operatorname{EXP}(-(\$ N 24+\$ L \$ 4) * \$ O \$ 5))\right)+\left((0.37 /(\$ N 24+\$ L \$ 6))^{*}(1-\operatorname{EXP}(-(\$ N 24+\$ L \$ 6) * \$ O \$ 5))\right)$ \\
\hline 26 & Pu-240 & $=\left(0.63 /(\$ N 26+\$ L \$ 4)^{*}\left(1-\operatorname{EXP}\left(-(\$ N 26+\$ L \$ 4)^{*} \$ 0 \$ 5\right)\right)\right)+\left((0.37 /(\$ N 26+\$ L \$ 6))^{*}\left(1-\operatorname{EXP}\left(-(\$ N 26+\$ L \$ 6)^{*} \$ 0 \$ 5\right)\right)\right)$ \\
\hline 27 & Pu-241 & $=\left(0.63 /(\$ N 27+\$ L \$ 4)^{*}\left(1-\operatorname{EXP}\left(-(\$ N 27+\$ L \$ 4)^{*} \$ O \$ 5\right)\right)\right)+\left((0.37 /(\$ N 27+\$ L \$ 6))^{*}\left(1-\operatorname{EXP}\left(-(\$ N 27+\$ L \$ 6)^{*} \$ 0 \$ 5\right)\right)\right)$ \\
\hline 28 & Pu pure-242 & $=\left(0.63 /(\$ N 28+\$ L \$ 4)^{*}\left(1-\operatorname{EXP}\left(-(\$ N 28+\$ L \$ 4)^{*} \$ O \$ 5\right)\right)\right)+\left((0.37 /(\$ N 28+\$ L \$ 6))^{*}\left(1-\operatorname{EXP}\left(-(\$ N 28+\$ L \$ 6)^{*} \$ O \$ 5\right)\right)\right)$ \\
\hline 29 & Sr-90 & $=\left(0.63 /(\$ N 29+\$ L \$ 4)^{*}(1-\operatorname{EXP}(-(\$ N 29+\$ L \$ 4) * \$ O \$ 5))\right)+\left((0.37 /(\$ N 29+\$ L \$ 6))^{*}\left(1-\operatorname{EXP}\left(-(\$ N 29+\$ L \$ 6)^{*} \$ 0 \$ 5\right)\right)\right)$ \\
\hline 30 & TC-99 & $=\left(0.63 /(\$ N 30+\$ L \$ 4)^{*}\left(1-\operatorname{EXP}\left(-(\$ N 30+\$ L \$ 4)^{*} \$ O \$ 5\right)\right)\right)+\left((0.37 /(\$ N 30+\$ L \$ 6))^{*}\left(1-\operatorname{EXP}\left(-(\$ N 30+\$ L \$ 6)^{*} \$ O \$ 5\right)\right)\right)$ \\
\hline 31 & Th-232 & $=\left(0.63 /(\$ N 31+\$ L \$ 4)^{*}\left(1-\operatorname{EXP}\left(-(\$ N 31+\$ L \$ 4)^{*} \$ O \$ 5\right)\right)\right)+\left((0.37 /(\$ N 31+\$ L \$ 6))^{*}\left(1-\operatorname{EXP}\left(-(\$ N 31+\$ L \$ 6)^{*} \$ O \$ 5\right)\right)\right)$ \\
\hline 32 & TI-204 & $=\left(0.63 /(\$ N 32+\$ L \$ 4)^{*}(1-\operatorname{EXP}(-(\$ N 32+\$ L \$ 4) * \$ O \$ 5))\right)+\left((0.37 /(\$ N 32+\$ L \$ 6))^{*}(1-E X P(-(\$ N 32+\$ L \$ 6) * \$ O \$ 5))\right)$ \\
\hline 33 & U-235 & $=\left(0.63 /(\$ N 33+\$ L \$ 4)^{*}\left(1-\operatorname{EXP}\left(-(\$ N 33+\$ L \$ 4)^{*} \$ O \$ 5\right)\right)\right)+\left((0.37 /(\$ N 33+\$ L \$ 6))^{*}\left(1-\operatorname{EXP}\left(-(\$ N 33+\$ L \$ 6)^{*} \$ O \$ 5\right)\right)\right)$ \\
\hline 34 & U-238 & $=\left(0.63 /(\$ N 34+\$ L \$ 4)^{*}\left(1-\operatorname{EXP}\left(-(\$ N 34+\$ L \$ 4)^{*} \$ O \$ 5\right)\right)\right)+\left((0.37 /(\$ N 34+\$ L \$ 6))^{*}\left(1-\operatorname{EXP}\left(-(\$ N 34+\$ L \$ 6)^{*} \$ O \$ 5\right)\right)\right)$ \\
\hline 35 & Total & \\
\hline 36 & $\mathrm{Ba}-137 \mathrm{~m}$ & $=\left(0.63 /(\$ N 36+\$ L \$ 4)^{*}\left(1-\operatorname{EXP}\left(-(\$ N 36+\$ L \$ 4)^{*} \$ O \$ 5\right)\right)\right)+\left((0.37 /(\$ N 36+\$ L \$ 6))^{*}\left(1-\operatorname{EXP}\left(-(\$ N 36+\$ L \$ 6)^{*} \$ O \$ 5\right)\right)\right)$ \\
\hline 37 & $\mathrm{Y}-90$ & $=\left(0.63 /(\$ N 37+\$ L \$ 4)^{*}\left(1-\operatorname{EXP}\left(-(\$ N 37+\$ L \$ 4)^{*} \$ O \$ 5\right)\right)\right)+\left((0.37 /(\$ N 37+\$ L \$ 6))^{*}\left(1-\operatorname{EXP}\left(-(\$ N 37+\$ L \$ 6)^{*} \$ O \$ 5\right)\right)\right)$ \\
\hline 38 & Pr-144 & $=\left(0.63 /(\$ N 38+\$ L \$ 4)^{*}\left(1-\operatorname{EXP}\left(-(\$ N 38+\$ L \$ 4)^{*} \$ O \$ 5\right)\right)\right)+\left((0.37 /(\$ N 38+\$ L \$ 6))^{*}(1-\operatorname{EXP}(-(\$ N 38+\$ L \$ 6) * \$ O \$ 5))\right)$ \\
\hline 39 & Pa-233 & $=\left(0.63 /(\$ N 39+\$ L \$ 4)^{*}\left(1-\operatorname{EXP}\left(-(\$ N 39+\$ L \$ 4)^{*} \$ O \$ 5\right)\right)\right)+\left((0.37 /(\$ N 39+\$ L \$ 6))^{*}\left(1-\operatorname{EXP}\left(-(\$ N 39+\$ L \$ 6)^{*} \$ 0 \$ 5\right)\right)\right)$ \\
\hline 40 & Th-234 & $=\left(0.63 /(\$ N 40+\$ L \$ 4)^{*}\left(1-\operatorname{EXP}\left(-(\$ N 40+\$ L \$ 4)^{*} \$ O \$ 5\right)\right)\right)+\left((0.37 /(\$ N 40+\$ L \$ 6))^{*}\left(1-\operatorname{EXP}\left(-(\$ N 40+\$ L \$ 6)^{*} \$ O \$ 5\right)\right)\right)$ \\
\hline 41 & Pa-234 & $=\left(0.63 /(\$ N 41+\$ L \$ 4)^{*}\left(1-\operatorname{EXP}\left(-(\$ N 41+\$ L \$ 4)^{*} \$ O \$ 5\right)\right)\right)+\left((0.37 /(\$ N 41+\$ L \$ 6))^{*}\left(1-\operatorname{EXP}\left(-(\$ N 41+\$ L \$ 6)^{*} \$ O \$ 5\right)\right)\right)$ \\
\hline 42 & Np-239 & $=\left(0.63 /(\$ N 42+\$ L \$ 4)^{*}\left(1-\operatorname{EXP}\left(-(\$ N 42+\$ L \$ 4)^{*} \$ 0 \$ 5\right)\right)\right)+\left((0.37 /(\$ N 42+\$ L \$ 6))^{*}(1-\operatorname{EXP}(-(\$ N 42+\$ L \$ 6) * \$ 0 \$ 5))\right)$ \\
\hline 43 & Th-231 & $=\left(0.63 /(\$ N 43+\$ L \$ 4)^{*}\left(1-\operatorname{EXP}\left(-(\$ N 43+\$ L \$ 4)^{*} \$ 0 \$ 5\right)\right)\right)+\left((0.37 /(\$ N 43+\$ L \$ 6))^{*}\left(1-\operatorname{EXP}\left(-(\$ N 43+\$ L \$ 6)^{*} \$ O \$ 5\right)\right)\right)$ \\
\hline 44 & Ra-228 & $=\left(0.63 /(\$ N 44+\$ L \$ 4)^{*}\left(1-\operatorname{EXP}\left(-(\$ N 44+\$ L \$ 4)^{*} \$ 0 \$ 5\right)\right)\right)+\left((0.37 /(\$ N 44+\$ L \$ 6))^{*}\left(1-\operatorname{EXP}\left(-(\$ N 44+\$ L \$ 6)^{*} \$ O \$ 5\right)\right)\right)$ \\
\hline 45 & AC-228 & $=\left(0.63 /(\$ N 45+\$ L \$ 4)^{*}\left(1-\operatorname{EXP}\left(-(\$ N 45+\$ L \$ 4)^{*} \$ 0 \$ 5\right)\right)\right)+\left((0.37 /(\$ N 45+\$ L \$ 6))^{*}\left(1-\operatorname{EXP}\left(-(\$ N 45+\$ L \$ 6)^{*} \$ O \$ 5\right)\right)\right)$ \\
\hline
\end{tabular}




\begin{tabular}{|c|c|c|}
\hline & $\bar{G}$ & $\bar{H}$ \\
\hline 53 & $5.85 E+00$ & DRL (uCi/m^2) - PAG/(CEDE+External) \\
\hline 54 & $4.34 E+00$ & DRL (uCi/m^2) - PAG/(BS Dose + External) \\
\hline 55 & $7.83 E+02$ & DRL (UCi/m^2) - PAG/(Skin Dose) \\
\hline
\end{tabular}




\begin{tabular}{|c|ll|}
\hline & \multicolumn{1}{|c|}{$G$} & \multicolumn{1}{c|}{} \\
\hline 53 & $=G 3 /(H 49+149)$ & DRL $\left(u C i / m^{\wedge} 2\right)-P A G /(C E D E+E x t e r n a l)$ \\
54 & $=G 4 /(L 49+149)$ & DRL $\left(u C i / m^{\wedge} 2\right)-P A G /(B S$ Dose + External) \\
\cline { 1 - 2 } 55 & $=G 5 / K 49$ & DRL $\left(u C i / m^{\wedge} 2\right)-P A G /($ Skin Dose $)$ \\
\hline
\end{tabular}




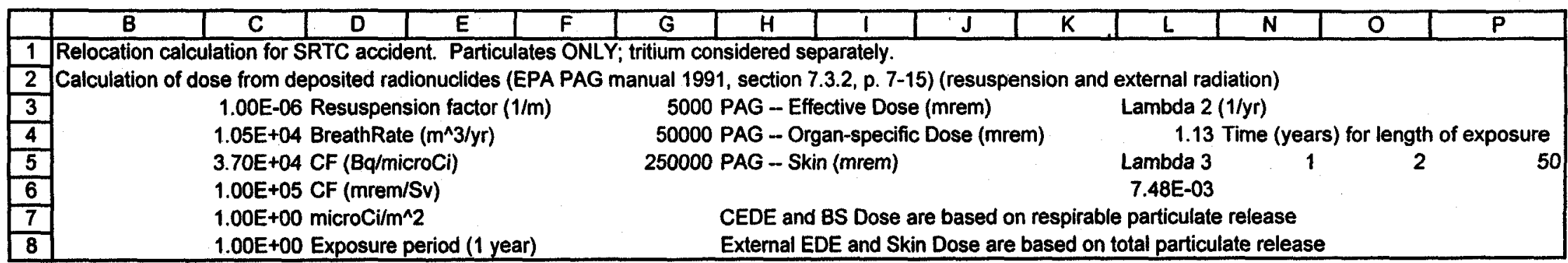




\begin{tabular}{|c|c|c|c|c|c|c|c|c|c|}
\hline & $\bar{B}$ & C & $\bar{D}$ & $\bar{G}$ & $\mathrm{H}$ & $\mathbf{L}$ & $\mathbf{N}$ & 0 & $\bar{P}$ \\
\hline 1 & \multirow{2}{*}{\multicolumn{9}{|c|}{ Relocation calculation for SRTC a }} \\
\hline 2 & & & & & & & & & \\
\hline 3 & & $=$ Relo_HIB3 & $=$ Relo_HIC3 & 5000 & =Relo_H!H3 & Lambda 2 (1/yr) & & & \\
\hline 4 & & $=$ Relo_H!B4 & $=$ Relo_H!C4 & 50000 & $=$ Relo_H!H4 & 1.13 & \multicolumn{3}{|l|}{ Time (years) for length } \\
\hline 5 & & $=$ Relo_HIB5 & $=$ Relo_H!C5 & $250000^{*}$ & $=$ Relo_H!H5 & Lambda $3(1 / y r)$ & 1 & 2 & 50 \\
\hline 6 & & $=$ Relo_H!B6 & $=$ Relo_H!C6 & & & 0.00748 & . & & \\
\hline 7 & & $=$ Relo_H!B7 & $=$ Relo_H!C7 & & CEDE and BS Dose a & & & & \\
\hline 8 & & $=$ Relo-H!B8 & $=$ Relo_H!C8 & & External EDE and Ski & & & & \\
\hline
\end{tabular}




\begin{tabular}{|c|c|c|c|}
\hline & $B$ & C & $\bar{D}$ \\
\hline 9 & Nuclide & $\begin{array}{l}\text { Total } \\
\text { Release } \\
\text { (Ci) }\end{array}$ & $\begin{array}{l}\text { Activity } \\
\text { fraction } \\
\text { (Total) }\end{array}$ \\
\hline 10 & Am-241 & 3.23E-02 & $1.09 \mathrm{E}-02$ \\
\hline 11 & Am-243 & $7.26 \mathrm{E}-04$ & $2.45 \mathrm{E}-0$. \\
\hline 12 & |Ba-133 & $4.04 \mathrm{E}-13$ & 1.36E-13 \\
\hline 13 & |ce-144 & 1.42E-05 & 4.80E-06 \\
\hline 14 & Cf-249 & 9.53E-06 & $3.22 E-06$ \\
\hline 15 & Cf-252 & $1.19 E-02$ & 4.03E-03 \\
\hline 16 & $\mathrm{Cm}-244$ & $1.53 \mathrm{E}-02$ & $5.15 \mathrm{E}-0 \mathrm{~s}$ \\
\hline 17 & $\mathrm{Cm}-246$ & $7.50 E-04$ & $2.53 E-04$ \\
\hline 18 & Co-60 & $40 \mathrm{E}-01$ & $1.82 \mathrm{E}-01$ \\
\hline 19 & Cs-1 & $91 \mathrm{E}-01$ & $3.01 E-01$ \\
\hline 20 & H-3 & $0.00 E+00$ & $0.00 E+O C$ \\
\hline 21 & Np-237 & 3.15E-05 & $1.06 \mathrm{E}-05$ \\
\hline 22 & $\mathrm{Pm}-147$ & $89 E-08$ & $6.38 \mathrm{E}-0 \mathrm{~s}$ \\
\hline 23 & Pu-238 & $15 \mathrm{E}-02$ & $1.40 E-0$ \\
\hline 24 & Pu-239 & $6.94 E-02$ & $2.34 \mathrm{E}-02$ \\
\hline 25 & Pu mix-239/241 & $0.00 E+00$ & $0.00 E+00$ \\
\hline 26 & Pu-240 & $1.59 \mathrm{E}-02$ & 5.37E-03 \\
\hline 27 & Pu-241 & $4.58 \mathrm{E}-01$ & $1.55 \mathrm{E}-01$ \\
\hline 28 & Pu pure-242 & 1.10E-04 & $3.70 E-0$ \\
\hline 29 & Sr-90 & $8.84 E-01$ & $2.99 \mathrm{E}-01$ \\
\hline 30 & TC-99 & $70 E-13$ & $9.12 \mathrm{E}-14$ \\
\hline 31 & Th-232 & $E-06$ & $4.90 E-0$ \\
\hline 32 & T1-20 & $75 \mathrm{E}-10$ & $2.28 \mathrm{E}-1$ \\
\hline 33 & U-235 & $.34 E-06$ & 4.52E-07 \\
\hline 34 & U-238 & $1.62 \mathrm{E}-04$ & 5.45E-05 \\
\hline 35 & Total & $2.96 \mathrm{E}+00$ & 1.00E+OC \\
\hline 36 & Ba-137m & $8.91 E-01$ & $3.01 E-0$ \\
\hline 37 & $Y-90$ & $E-01$ & $2.99 \mathrm{E}-0$ \\
\hline 38 & Pr-144 & $1.42 E-05$ & 4.80E-06 \\
\hline 39 & $\mathrm{~Pa}-233$ & $15 E-05$ & $1.06 \mathrm{E}-05$ \\
\hline 40 & Th-2: & $=-04$ & 5.45E-05 \\
\hline 41 & Pa-234 & $1.62 \mathrm{E}-04$ & 5.45E-05 \\
\hline 42 & Np-239 & $7.26 \mathrm{E}-04$ & $2.45 E-04$ \\
\hline 43 & Th-231 & $1.34 \mathrm{E}-06$ & $4.52 E-07$ \\
\hline 44 & Ra-228 & $1.45 \mathrm{E}-06$ & 4.90E-C \\
\hline & AC-228 & $1.45 \mathrm{E}-06$ & 4.90E-07 \\
\hline
\end{tabular}




\begin{tabular}{|c|c|c|c|}
\hline & B & $\mathrm{C}$ & D \\
\hline 9 & Nuclide & Total Release (Ci) & $\begin{array}{l}\text { Activity fraction } \\
\text { (Total) }\end{array}$ \\
\hline 10 & Am-241 & $={ }^{\prime C}$ : $\mid$ EP_relocation $\left.Y S o u r c e S R T C . x \mid s\right]$ resp sums $\mid \$ H 10$ & $=\mathrm{C} 10 / \$ \mathrm{C} \$ 35$ \\
\hline 11. & Am-243 & $={ }^{\prime} \mathrm{C}:$ IEP_relocationYSourceSRTC .xIs]resp sums'!\$H11 & $=\mathrm{C} 11 / \$ \mathrm{C} \$ 35$ \\
\hline 12 & $\mathrm{Ba}-133$ & $=' C:$ :LE_relocationYSourceSRTC.xIs]resp sums'!\$H12 & $=\mathrm{C} 12 / \$ \mathrm{C} \$ 35$ \\
\hline 13 & $\mathrm{Ce}-144$ & $=' C:$ IEP_relocationVSourceSRTC.xIs]resp sums'!\$H13 & $=\mathrm{C} 13 / \$ \mathrm{C} \$ 35$ \\
\hline 14 & Cf-249 & $=' C:$ IEP_relocationYSourceSRTC.xls]resp sums'!\$H14 & $=C 14 / \$ C \$ 35$ \\
\hline 15 & Cf-252 & ='C:IEP_relocationYSourceSRTC.xls]resp sums'!\$H15 & $=\mathrm{C} 15 / \$ \mathrm{C} \$ 35$ \\
\hline 16 & $\mathrm{Cm}-244$ & $=' C:$ IEP_relocation YSourceSRTC.xIs]resp sums'!\$H16 & $=C 16 / \$ C \$ 35$ \\
\hline 17 & $\mathrm{Cm}-246$ & ='C:IEP_relocationYSourceSRTC.xis]resp sums'!\$H17 & $=C 17 / \$ C \$ 35$ \\
\hline 18 & Co-60 & ='C:LEP_relocationYSourceSRTC.xls]resp sums'!\$H18 & $=C 18 / \$ C \$ 35$ \\
\hline 19 & Cs-137 & ='C:IEP_relocation YSourceSRTC.xis]resp sums'!\$H19 & $=\mathrm{C} 19 / \$ \mathrm{C} \$ 35$ \\
\hline 20 & $\mathrm{H}-3$ & 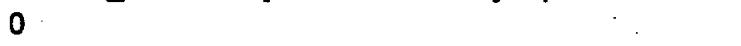 & $=\mathrm{C} 20 / \$ C \$ 35$ \\
\hline 21 & Np-237 & $=' C: \mid$ IEP_relocationYSourceSRTC.x|s]resp sums' $\mid \$ H 21$ & $=C 21 / \$ C \$ 35$ \\
\hline 22 & $P m-147$ & ='C:LEP_relocationYSourceSRTC.x|s]resp sums'!\$H22 & $=\mathrm{C} 22 / \$ C \$ 35$ \\
\hline 23. & Pu-238 & ='C:IEP_relocationYSourceSRTC.x|s]resp sums'!\$H23 & $=\mathrm{C} 23 / \$ C \$ 35$ \\
\hline 24 & Pu-239 & ='C:IEP_relocation YSourceSRTC.x|s]resp sums'!\$H24 & $=\mathrm{C} 24 / \$ \mathrm{C} \$ 35$ \\
\hline 25 & Pu mix-239/241 & ='C:LEP_relocationYSourceSRTC.x|s]resp sums'!\$H25 & $=C 25 / \$ C \$ 35$ \\
\hline 26 & Pu-240 & ='C:IEP_relocationVSourceSRTC.xis]resp sums'!\$H26 & $=\mathrm{C} 26 / \$ \mathrm{C} \$ 35$ \\
\hline 27. & Pu-241 & ='C:IEP_relocationYSSourceSRTC.xIs]resp sums' $\mid \$ H 27$ & $=C 27 / \$ C \$ 35$ \\
\hline 28 & Pu pure-242 & ='C:IEP_relocationYSourceSRTC.xls]resp sums'!\$H28 & $=\mathrm{C} 28 / \$ \mathrm{C} \$ 35$ \\
\hline 29 & Sr-90 & $={ }^{\prime} C$ :LEP_relocation $Y$ SourceSRTC.xis]resp sums' $\$$ H29 & $=\mathrm{C} 29 / \$ \mathrm{C} \$ 35$ \\
\hline 30 & Tc-99 & ='C:IEP_relocationYSourceSRTC.xIs]resp sums'!\$H30 & $=\mathrm{C} 30 / \$ C \$ 35$ \\
\hline 31. & Th-232 & ='C:IEP_relocationYSourceSRTC.xls]resp sums'!\$H31 & $=C 31 / \$ C \$ 35$ \\
\hline 32 & TI-204 & ='C:IEP_relocationVSourceSRTC.xIs]resp sums'!\$H32 & $=C 32 / \$ C \$ 35$ \\
\hline 33 & $\mathrm{U}-235$ & ='C:IEP_relocationYSourceSRTC.xis]resp sums'!\$H33 & $=\mathrm{C} 33 / \$ \mathrm{C} \$ 35$ \\
\hline 34 & $\mathrm{U}-238$ & $={ }^{\prime} \mathrm{C}:$ :LP_relocationYSourceSRTC.xls]resp sums'!\$H34 & $=\mathrm{C} 34 / \$ C \$ 35$ \\
\hline 35 & Total & $=S U M(C 10: C 34)$ & $=\operatorname{SUM}(\mathrm{D} 10: \mathrm{D} 34)$ \\
\hline 36 & $\mathrm{Ba}-137 \mathrm{~m}$ & $=\mathrm{C} 19$ & $=\mathrm{D} 19$ \\
\hline 37 & $Y-90$ & $=\mathrm{C} 29$ & $=\mathrm{D} 29$ \\
\hline 38 & Pr-144 & $=\mathrm{C} 13$ & $=\mathrm{D} 13$ \\
\hline 39 & Pa-233 & $=\mathrm{C} 21$ & $=\mathrm{D} 21$ \\
\hline 40 & Th-234 & $=\mathrm{C} 34$ & $=\mathrm{D} 34$ \\
\hline 41 & Pa-234 & $=\mathrm{C} 34$ & $=\mathrm{D} 34$ \\
\hline 42 & Np-239 & $=\mathrm{C} 11$ & $=D 11$ \\
\hline 43 & Th-231 & $=\mathrm{C} 33$ & $=\mathrm{D} 33$ \\
\hline 44 & Ra-228 & $=C 31$ & $=\mathrm{D} 31$ \\
\hline 45 & $A C-228$ & $=\mathrm{C} 31$ & $=\mathrm{D} 31$ \\
\hline
\end{tabular}




\begin{tabular}{|c|c|c|c|}
\hline & $B$ & $E$ & $F$ \\
\hline 9 & Nuclide & $\begin{array}{l}\text { Respirable } \\
\text { Release } \\
\text { (Ci) }\end{array}$ & $\begin{array}{l}\text { Activity } \\
\text { fraction } \\
\text { (Resp) }\end{array}$ \\
\hline 10 & Am-241 & $1.49 E-02$ & $5.56 \mathrm{E}-0$ \\
\hline 11 & Am-243 & 4.55E-04 & $1.70 E-04$ \\
\hline 12 & Ba-133 & $4.04 \mathrm{E}-13$ & $1.51 E-13$ \\
\hline 13 & $\mathrm{Ce}-144$ & $1.42 E-05$ & $5.32 E-06$ \\
\hline 14 & Cf-249 & $8.24 E-06$ & $3.08 E-0$ \\
\hline 15 & Cf-252 & 1.19E-02 & 4.47E-03 \\
\hline 16 & $\mathrm{Cm}-244$ & $1.71 E-02$ & $6.40 E-03$ \\
\hline 17 & $\mathrm{Cm}-246$ & 4.99E-04 & $1.87 E-04$ \\
\hline 18 & $C_{0}-60$ & 40E-01 & $2.02 E-01$ \\
\hline 19 & Cs-137 & $8.91 E-01$ & 3.34E-01 \\
\hline 20 & $\mathrm{H}-3$ & $0.00 E+00$ & $0.00 E+00$ \\
\hline 21 & Np-237 & 2.44E-05 & $9.14 E-06$ \\
\hline 22 & Pm-147 & $9 E-08$ & $7.07 \mathrm{E}-0$ \\
\hline 23 & Pu-238 & $1.81 E-02$ & $6.77 \mathrm{E}-03$ \\
\hline 24 & Pu-239 & 2.35E-02 & $8.78 E-03$ \\
\hline 25 & Pu mix-239/241 & $0.00 E+00$ & $0.00 E+00$ \\
\hline 26 & Pu-240 & $5.34 \mathrm{E}-03$ & $2.00 E-0$ \\
\hline 27 & Pu-241 & $2.66 \mathrm{E}-01$ & $9.97 E-02$ \\
\hline 28 & Pu pure-242 & 3.47E-05 & 1.30E-05 \\
\hline 29 & Sr-90 & $3 E-01$ & 3.30E-01 \\
\hline 30 & TC-99 & $2.70 E-13$ & $1.01 E-13$ \\
\hline 31 & Th-232 & $1.44 E-06$ & 5.38E-07 \\
\hline 32 & TI-204 & $5 E-10$ & 2.53E-10 \\
\hline 33 & U-23: & $3 E-07$ & 2.57E-07 \\
\hline 34 & U-238 & $.11 E-05$ & $1.91 E-05$ \\
\hline 35 & Total & $2.67 E+00$ & $1.00 E+00$ \\
\hline 36 & Ba-137m & 8.91E-01 & 3.34E-01 \\
\hline 37 & $Y-90$ & 8.83E-01 & 3.30E-01 \\
\hline 38 & Pr-14 & $1.42 E-05$ & $5.32 E-0$ \\
\hline 39 & $\mathrm{~Pa}-233$ & 2.44E-05 & $9.14 \mathrm{E}-\mathrm{OC}$ \\
\hline 40 & Th-234 & 5.11E-05 & $1.91 E-05$ \\
\hline 41 & Pa-234 & 5.11E-05 & $1.91 E-0$ \\
\hline 42 & Np-239 & 4.55E-04 & $1.70 E-0$ \\
\hline 43 & Th-231 & $6.88 E-07$ & $2.57 E-0$ \\
\hline 44 & Ra-228 & $1.44 \mathrm{E}-06$ & $5.38 E-07$ \\
\hline 45 & Ac-228 & $1.44 E-06$ & $5.38 E-07$ \\
\hline
\end{tabular}




\begin{tabular}{|c|c|c|c|}
\hline & $\bar{B}$ & $E$ & $\mathbf{F}$ \\
\hline 9 & Nuclide & Respirable Release (Ci) & $\begin{array}{l}\text { Activity fraction } \\
\text { (Resp) }\end{array}$ \\
\hline 10 & Am-241 & $={ }^{\prime} C$ :IEP_relocationYSourceSRTC.xIs]resp sums'!\$F10 & $=E 10 / \$ E \$ 35$ \\
\hline 11 & Am-243 & ='C:LEP_relocationYSourceSRTC.xIs]resp sums'!\$F11 & $=\mathrm{E} 11 / \$ \mathrm{E} \$ 35$ \\
\hline 12 & Ba-133 & ='C:IEP_relocationySourceSRTC.xIs]resp sums'!\$F12 & $=E 12 / \$ E \$ 35$ \\
\hline 13 & Ce-144 & $=' C:$ IEP_relocation VSourceSRTC.xIs]resp sums'!\$F13 & $=E 13 / \$ E \$ 35$ \\
\hline 14 & Cf-249 & ='C:IEP_relocationYSourceSRTC.xIs]resp sums'!\$F14 & $=E 14 / \$ E \$ 35$ \\
\hline 15 & Cf-252 & $={ }^{\prime} C:$ IEP_relocation YSourceSRTC.xis]resp sums'!\$F15 & $=E 15 / \$ E \$ 35$ \\
\hline 16 & $\mathrm{Cm}-244$ & ='C:IEP_relocationYSourceSRTC.xis\}resp sums'!\$F16 & $=E 16 / \$ E \$ 35$ \\
\hline 17 & $\mathrm{Cm}-246$ & $=' C$ :IEP_relocationYSourceSRTC.xls]resp sums'!\$F17 & $=E 17 / \$ E \$ 35$ \\
\hline 18 & Co-60 & ='C:IEP_relocation\SourceSRTC.xls]resp sums'!\$F18 & $=E 18 / \$ E \$ 35$ \\
\hline 19 & Cs-137 & ='C:IEP_relocationVSSourceSRTC.xls]resp sums'!\$F19 & $=E 19 / \$ E \$ 35$ \\
\hline 20 & $\mathrm{H}-3$ & 0 & $=E 20 / \$ E \$ 35$ \\
\hline 21 & Np-237 & $={ }^{\prime} C$ :IEP_relocationYSourceSRTC.xls]resp sums'!\$F21 & $=E 21 / \$ E \$ 35$ \\
\hline 22 & Pm-147 & $={ }^{\prime} C:$ IEP_relocation $\backslash$ SourceSRTC.xls]resp sums' $\$$ FF22 & $=E 22 / \$ E \$ 35$ \\
\hline 23 & Pu-238 & ='C:IEP_relocationy[SourceSRTC.xis]resp sums'!\$F23 & $=E 23 / \$ E \$ 35$ \\
\hline 24 & Pu-239 & $=' C:$ IEP_relocationUYSourceSRTC.xIs]resp sums'!\$F24 & $=E 24 / \$ E \$ 35$ \\
\hline 25 & Pu mix-239/241 & ='C:IEP_relocationYSourceSRTC.xls]resp sums'I\$F25 & $=E 25 / \$ E \$ 35$ \\
\hline 26 & Pu-240 & $=$ 'C:IEP_relocationVSourceSRTC.xis]resp sums'!\$F26 & $=E 26 / \$ E \$ 35$ \\
\hline 27 & Pu-241 & ='C:IEP_relocationYSourceSRTC.x|s]resp sums'!\$F27 & $=E 27 / \$ E \$ 35$ \\
\hline 28 & Pu pure-242 & $={ }^{\prime} C:$ IEP_relocationViSourceSRTC.xis]resp sums'!\$F28 & $=E 28 / \$ E \$ 35$ \\
\hline 29 & Sr-90 & ='C:IEP_relocationYSourceSRTC.xis]resp sums'!\$F29 & $=E 29 / \$ E \$ 35$ \\
\hline 30 & TC-99 & ='C:IEP_relocationYSourceSRTC.xis]resp sums'!\$F30 & $=E 30 / \$ E \$ 35$ \\
\hline 31 & Th-232 & $=' C:$ IEP_relocationVSourceSRTC.x|s]resp sums'!\$F31 & $=E 31 / \$ E \$ 35$ \\
\hline 32 & T1-204 & ='C:IEP_relocationYSourceSRTC.xis]resp sums'!\$F32 & $=E 32 / \$ E \$ 35$ \\
\hline 33 & U-235 & ='C:IEP_relocationYSourceSRTC.xIs]resp sums'!\$F33 & $=$ E33/\$E $\$ 35$ \\
\hline 34 & U-238 & $=' C:$ IEP_relocationYSourceSRTC.x|s]resp sums'!\$F34 & $=E 34 / \$ E \$ 35$ \\
\hline 35 & Total & $=$ SUM(E10:E34) & $=\operatorname{SUM}(F 10: F 34)$ \\
\hline 36 & $B a-137 m$ & $=\mathrm{E} 19$ & $=F 19$ \\
\hline 37 & $Y-90$ & $=E 29$ & $=F 29$ \\
\hline 38 & $\operatorname{Pr}-144$ & $=E 13$ & $=\mathrm{F} 13$ \\
\hline 39 & $\mathrm{~Pa}-233$ & $=E 21$ & $=F 21$ \\
\hline 40 & Th-234 & $=E 34$ & $=\mathbf{F} 34$ \\
\hline 41 & Pa-234 & $=E 34$ & $=\mathbf{F} 34$ \\
\hline 42 & Np-239 & $=\mathrm{E} 11$ & $=F 11$ \\
\hline 43 & Th-231 & $=E 33$ & $=F 33$ \\
\hline 44 & Ra-228 & $=E 31$ & $=F 31$ \\
\hline 45 & Ac-228 & $=E 31$ & $=F 31$ \\
\hline
\end{tabular}




\begin{tabular}{|c|c|c|c|}
\hline & $\bar{B}$ & $\bar{H}$ & T \\
\hline 9 & Nuclide & $\begin{array}{l}\text { CEDE (mrem per } \\
\mathrm{uCl} / \mathrm{m}^{\wedge} 2 \text { ) }\end{array}$ & $\begin{array}{l}\text { External EDE } \\
\text { (mrem per } \\
u\left(\mathrm{i} / \mathrm{m}^{\wedge} 2\right)\end{array}$ \\
\hline 10 & Am-241 & $4.01 E+02$ & $\quad 5.59 \mathrm{E}-01$ \\
\hline 11 & $A m-243$ & $1.26 \mathrm{E}+01$ & 2.87E-02 \\
\hline 12 & Ba-133 & $6.73 E-14$ & 3.44E-11 \\
\hline 13 & $\mathrm{Ce}-144$ & 1.51E-05 & 8.32E-06 \\
\hline 14 & CF-249 & $2.86 E=01$ & $1.92 E-03$ \\
\hline 15 & C $F-252$ & $1.34 E+01$ & 5.17E-04 \\
\hline 16 & $\mathrm{Cm}-244$ & $1.28 E+02$ & $3.63 E-03$ \\
\hline 17 & $\mathrm{Cm}-246$ & 1.41E+01 & $3.29 E-04$ \\
\hline 18 & Co-60 & $1.46 \mathrm{E}+00$ & $1.45 E+02$ \\
\hline 19 & Cs-137 & 1.12E+00 & $0.00 E+00$ \\
\hline 20 & $\mathrm{H}-3$ & $0.00 E+00$ & $0.00 E+00$ \\
\hline 21 & $\mathrm{~Np}-237$ & 8.30E-01 & $6.11 E-04$ \\
\hline 22 & $P m-147$ & 5.28E-09 & 5.25E-12 \\
\hline 23 & $P u-238$ & $3.75 E+02$ & $1.79 E-02$ \\
\hline 24 & Pu-239 & $6.33 E+02$ & 1.57E-02 \\
\hline 25 & Pu mix-239/241 & & \\
\hline 26 & Pu-240 & $1.44 E+02$ & 7.79E-03 \\
\hline 27 & $P u-241$ & $5.52 E+01$ & $0.00 E+00$ \\
\hline 28 & Pu pure-242 & 8.95E-01 & 4.47E-05 \\
\hline 29 & Sr-90 & $4.39 E+01$ & $0.00 E+00$ \\
\hline 30 & Tc-99 & $1.41 E-13$ & $1.01 E-16$ \\
\hline 31 & Th-232 & $1.48 E-01$ & $5.78 E-07$ \\
\hline 32 & $\pi /-204$ & $1.55 \mathrm{E}-11$ & $7.98 E-11$ \\
\hline 33 & U-235 & 5.31E-03 & 1.37E-04 \\
\hline 34 & U-238 & 3.80E-01 & $6.24 E-05$ \\
\hline 35 & Total & & \\
\hline 36 & Ba-137m & & $1.43 E-04$ \\
\hline 37 & $Y-90$ & & $0.00 E+00$ \\
\hline 38 & Pr-144 & & $7.52 E-10$ \\
\hline 39 & Pa-233 & & 2.77E-05 \\
\hline 40 & Th-234 & & 5.46E-06 \\
\hline 41 & Pa-234 & & 1.31E-05 \\
\hline 42 & $\mathrm{~Np}-239$ & & $4.82 E-05$ \\
\hline 43 & Th-231 & & 4.01E-09 \\
\hline 44 & Ra-228 & & 1.23E-13 \\
\hline 45 & Ac-228 & & $4.98 E-08$ \\
\hline$\frac{46}{47}$ & Total & $1.82 E+03$ & $1.45 E+02$ \\
\hline
\end{tabular}




\begin{tabular}{|c|c|c|c|}
\hline & B & $\mathrm{H}$ & 1 \\
\hline 9 & Nuclide & CEDE (mrem per $u C l / m^{\wedge} 2$ ) & External EDE (mrem per uCi/m^2) \\
\hline 10 & Am-241 & $=\$ C \$ 7^{*} \$ C \$ 3^{*} \$ C \$ 4^{*} \$ C \$ 5^{*} \$ C \$ 6^{*} F 10^{*}$ Relocation!H10*Q10 & $=\$ C \$ 7^{*} \$ D 10^{*}$ Relocation!E10*\$C\$35/\$E\$35*Q10 \\
\hline 11 & Am-243 & $=\$ C \$ 7^{*} \$ C \$ 3^{*} \$ C \$ 4^{*} \$ C \$ 5^{*} \$ C \$ 6^{*} F 11^{*}$ Relocation!H11*Q11 & $=\$ C \$ 7^{*} \$ D 11^{*}$ Relocation!E11*\$C $\$ 35 / \$ E \$ 35^{*} Q 11$ \\
\hline 12 & Ba- 133 & $=\$ C \$ 7^{*} \$ C \$ 3^{*} \$ C \$ 4^{*} \$ C \$ 5^{*} \$ C \$ 6^{*} F 12^{*}$ Relocation!H12*Q12 & $=\$ C \$ 7^{*} \$ D 12^{*}$ Relocation!E12*\$C\$35/\$E\$35*Q12 \\
\hline 13 & $\mathrm{Ce}-144$ & $=\$ C \$ 7^{*} \$ C \$ 3^{*} \$ C \$ 4^{*} \$ C \$ 5^{*} \$ C \$ 6^{*} F 13^{*}$ Relocation!H13*Q13 & $=\$ C \$ 7^{*} \$ D 13^{*}$ Relocation!E13*\$C $\$ 35 / \$ E \$ 35^{*} Q 13$ \\
\hline 14 & Cf-249 & $=\$ C \$ 7^{*} \$ C \$ 3^{*} \$ C \$ 4^{*} \$ C \$ 5^{*} \$ C \$ 6^{*} F 14^{*}$ Relocation!H14*Q14 & $=\$ C \$ 7^{\star} \$ D 14 *$ Relocation!E14"\$C $\$ 35 / \$ E \$ 35^{*} Q 14$ \\
\hline 15 & CF-252 & $=\$ C \$ 7^{*} \$ C \$ 3^{*} \$ C \$ 4^{*} \$ C \$ 5^{*} \$ C \$ 6^{*} F 15^{*}$ Relocation!H15*Q15 & $=\$ C \$ 7^{*} \$ D 15^{*}$ Relocation!E15*\$C $\$ 35 / \$ E \$ 35^{*} Q 15$ \\
\hline 16 & $\mathrm{Cm}-244$ & $=\$ C \$ 7^{\star} \$ C \$ 3^{*} \$ C \$ 4^{*} \$ C \$ 5^{*} \$ C \$ 6^{*} F 16^{*}$ Relocation!H16*Q16 & $=\$ C \$ 7^{*} \$ D 16^{*}$ Relocation!E16*\$C $\$ 35 / \$ E \$ 35^{*} Q 16$ \\
\hline 17 & $\mathrm{Cm}-246$ & $=\$ C \$ 7^{*} \$ C \$ 3^{*} \$ C \$ 4^{*} \$ C \$ 5^{*} \$ C \$ 6^{*} F 17^{*}$ Relocation!H17*Q17 & $=\$ C \$ 7^{*} \$ D 17^{*}$ Relocation!E17*\$C\$35/\$E\$35*Q17 \\
\hline 18 & Co-60 & $=\$ C \$ 7^{*} \$ C \$ 3^{*} \$ C \$ 4^{*} \$ C \$ 5^{*} \$ C \$ 6^{*} F 18^{*}$ Relocation!H18*Q18 & $=\$ C \$ 7^{*} \$ D 18^{*}$ Relocation!E18*\$C\$35/\$E $\$ 35^{*} Q 18$ \\
\hline 19 & Cs-137 & $=\$ C \$ 7^{*} \$ C \$ 3^{*} \$ C \$ 4^{*} \$ C \$ 5^{*} \$ C \$ 6^{*} F 19^{*}$ Relocation!H19*Q19 & $=\$ C \$ 7^{*} \$ D 19^{*}$ Relocation!E19*\$C\$35/\$E $\$ 35^{*} Q 19$ \\
\hline 20 & $\mathrm{H}-3$ & $=\$ C \$ 7^{\star} \$ C \$ 3^{*} \$ C \$ 4^{*} \$ C \$ 5^{*} \$ C \$ 6^{*} F 20^{*}$ Relocation!H20*Q20 & $=\$ C \$ 7^{*} \$ D 20^{*}$ Relocation!E $20^{*} \$ C \$ 35 / \$ E \$ 35^{*} Q 20$ \\
\hline 21 & Np-237 & $=\$ C \$ 7^{*} \$ C \$ 3^{*} \$ C \$ 4^{*} \$ C \$ 5^{*} \$ C \$ 6^{*} F 21^{*}$ Relocation!H21*Q21 & $=\$ C \$ 7^{*} \$ D 21^{*}$ Relocation!E21*\$C\$35/\$E\$35*Q21 \\
\hline 22 & Pm-147 & $=\$ C \$ 7^{*} \$ C \$ 3^{*} \$ C \$ 4^{*} \$ C \$ 5^{*} \$ C \$ 6^{*} F 22^{*}$ Relocation!H22*Q22 & $=\$ C \$ 7^{*} \$ D 22^{*}$ Relocation!E22*\$C\$35/\$E $\$ 35^{*} Q 22$ \\
\hline 23 & Pu-238 & $=\$ C \$ 7^{*} \$ C \$ 3^{*} \$ C \$ 4^{*} \$ C \$ 5^{*} \$ C \$ 6^{*} F 23^{*}$ Relocation!H23*Q23 & $=\$ C \$ 7^{*} \$ D 23^{*}$ Relocation!E23*\$C\$35/\$E $\$ 35^{*} \mathrm{Q} 23$ \\
\hline 24 & Pu-239 & $=\$ C \$ 7^{*} \$ C \$ 3^{*} \$ C \$ 4^{*} \$ C \$ 5^{*} \$ C \$ 6^{*} F 24^{*}$ Relocation!H24*Q24 & $=\$ C \$ 7^{*} \$ D 24^{*}$ Relocation!E24*\$C\$35/\$E $\$ 35^{*} Q 24$ \\
\hline 25 & Pu mix-239/24 & & \\
\hline 26 & Pu-240 & $=\$ C \$ 7^{*} \$ C \$ 3^{*} \$ C \$ 4^{*} \$ C \$ 5^{*} \$ C \$ 6^{*} F 26^{*}$ Relocation!H26*Q26 & $=\$ C \$ 7^{\star} \$ D 26^{*}$ Relocation!E26*\$C $\$ 35 / \$ E \$ 35^{*} Q 26$ \\
\hline 27 & Pu-241 & $=\$ C \$ 7^{*} \$ C \$ 3^{*} \$ C \$ 4^{*} \$ C \$ 5^{*} \$ C \$ 6^{*} F 27^{*}$ Relocation!H27*Q27 & $=\$ C \$ 7^{*} \$ D 27^{*}$ Relocation!E27*\$C\$35/\$E $\$ 35^{*} Q 27$ \\
\hline 28 & Pu pure-242 & $=\$ C \$ 7^{*} \$ C \$ 3^{*} \$ C \$ 4^{*} \$ C \$ 5^{*} \$ C \$ 6^{*}$ F $28^{*}$ Relocation!H28*Q28 & $=\$ C \$ 7^{*} \$ D 28^{*}$ Relocation!E28*\$C $\$ 35 / \$ E \$ 35^{*} Q 28$ \\
\hline 29 & Sr-90 & $=\$ C \$ 7^{*} \$ C \$ 3^{*} \$ C \$ 4^{*} \$ C \$ 5^{*} \$ C \$ 6^{*} F 29^{*}$ Relocation!H29*Q29 & $=\$ C \$ 7^{*} \$ D 29^{*}$ Relocation!E29*\$C\$35/\$E $\$ 35^{*} Q 29$ \\
\hline 30 & TC-99 & $=\$ C \$ 7^{*} \$ C \$ 3^{*} \$ C \$ 4^{*} \$ C \$ 5^{*} \$ C \$ 6^{*} F 30^{*}$ Relocation!H30*Q30 & $=\$ C \$ 7^{*} \$ D 30^{*}$ Relocation!E30*\$C\$35/\$E\$35*Q30 \\
\hline 31 & Th-232 & $=\$ C \$ 7^{*} \$ C \$ 3^{*} \$ C \$ 4^{*} \$ C \$ 5^{*} \$ C \$ 6^{*} F 31^{*}$ Relocation!H31*Q31 & $=\$ C \$ 7^{*} \$ D 31^{*}$ Relocation!E31*\$C $\$ 35 / \$ E \$ 35^{*} Q 31$ \\
\hline 32 & TI-204 & $=\$ C \$ 7^{*} \$ C \$ 3^{*} \$ C \$ 4^{*} \$ C \$ 5^{*} \$ C \$ 6^{*} \mathrm{~F} 32^{*}$ Relocation!H32*Q32 & $=\$ C \$ 7^{*} \$ D 32^{*}$ Relocation!E32*\$C\$35/\$E $\$ 35^{*} Q 32$ \\
\hline 33 & $\mathrm{U}-235$ & $=\$ C \$ 7^{*} \$ C \$ 3^{*} \$ C \$ 4^{*} \$ C \$ 5^{*} \$ C \$ 6^{*} F 33^{*}$ Relocation!H33* $Q 33$ & $=\$ C \$ 7^{*} \$ D 33^{*}$ Relocation!E33*\$C\$35/\$E $\$ 35^{*} Q 33$ \\
\hline 34 & ]U-238 & $=\$ C \$ 7^{*} \$ C \$ 3^{*} \$ C \$ 4^{*} \$ C \$ 5^{*} \$ C \$ 6^{*} F 34^{*}$ Relocation!H34*Q34 & $=\$ C \$ 7^{*} \$ D 34^{*}$ Relocation!E34*\$C\$35/\$E \$35*Q34 \\
\hline 35 & Total & & \\
\hline 36 & $\mathrm{Ba}-137 \mathrm{~m}$ & & $=\$ C \$ 7^{*} \$ D 36^{*}$ Relocation!E36*\$C $\$ 35 / \$ E \$ 35^{*} Q 36$ \\
\hline 37 & $\mathrm{Y}-90$ & & $=\$ C \$ 7^{*} \$ D 37^{*}$ Relocation!E37*\$C $\$ 35 / \$ E \$ 35^{*} Q 37$ \\
\hline 38 & Pr-144 & & $=\$ C \$ 7^{*} \$ D 38^{*}$ Relocation!E38*\$C $\$ 35 / \$ E \$ 35^{*} Q 38$ \\
\hline 39 & Pa-233 & & $=\$ C \$ 7^{*} \$ D 39^{*}$ Relocation!E39*\$C $\$ 35 / \$ E \$ 35^{*} Q 39$ \\
\hline 40 & Th-234 & & $=\$ C \$ 7^{*} \$ D 40^{*}$ Relocation!E40*\$C\$35/\$E $\$ 35^{*} Q 40$ \\
\hline 41 & Pa-234 & & $=\$ C \$ 7^{*} \$ D 41^{*}$ Relocation!E41*\$C $\$ 35 / \$ E \$ 35^{*} Q 41$ \\
\hline 42 & Np-239 & & $=\$ C \$ 7^{*} \$ D 42^{*}$ Relocation!E42*\$C $\$ 35 / \$ E \$ 35^{*} Q 42$ \\
\hline 43 & Th-231 & & $=\$ C \$ 7^{*} \$ D 43^{*}$ Relocation!E43*\$C\$35/\$E\$35*Q43 \\
\hline 44 & Ra-228 & & $=\$ C \$ 7^{*} \$ D 44^{*}$ Relocation!E44*\$C $\$ 35 / \$ E \$ 35^{*} Q 44$ \\
\hline 45 & AC-228 & & $=\$ C \$ 7^{\star} \$ D 45^{\star}$ Relocation!E45 $\$ C \$ 35 / \$ E \$ 35^{*} Q 45$ \\
\hline 46 & & & \\
\hline 47 & Total & $=S U M(H 10: H 46)$ & $=S U M(110: 146)$ \\
\hline
\end{tabular}




\begin{tabular}{|c|c|c|c|}
\hline & B & $\bar{K}$ & $L$ \\
\hline 9 & Nuclide & $\begin{array}{l}\text { Skin Dose } \\
\text { (mrem per } \\
\mathrm{uCi} / \mathrm{m}^{\wedge} 2 \text { ) }\end{array}$ & $\begin{array}{l}\text { BS Dose } \\
\text { (mrem per } \\
\mathrm{uCl} / \mathrm{m}^{\wedge} 2 \text { ) }\end{array}$ \\
\hline 10 & Am-241 & $1.20 E+\infty 0$ & $7.25 E+03$ \\
\hline 11 & Am-243 & $4.60 E-02$ & $2.29 E+02$ \\
\hline 12 & $B a-133$ & $4.78 \mathrm{E}-11$ & $3.03 E-13$ \\
\hline 13 & Ce-144 & 1.10E-05 & 7.06E-07 \\
\hline 14 & Cf-249 & $2.60 \mathrm{E}-03$ & $6.09 E+00$ \\
\hline 15 & Cf-252 & $3.80 \mathrm{E}-03$ & $4.34 E+01$ \\
\hline 16 & $\mathrm{Cm}-244$ & 2.87E-02 & $2.23 E+03$ \\
\hline 17 & $\mathrm{Cm}-246$ & $2.61 E-03$ & $2.57 E+02$ \\
\hline 18 & Co-60 & $1.94 E+02$ & $3.34 E-01$ \\
\hline 19 & Cs-137 & $9.40 E+01$ & $1.03 E+00$ \\
\hline 20 & $H-3$ & $0.00 E+00$ & $0.00 E+00$ \\
\hline 21 & Np-237 & $1.42 E-03$ & $1.86 E+01$ \\
\hline 22 & $P m-147$ & $6.36 \mathrm{E}-12$ & $1.00 \mathrm{E}-08$ \\
\hline 23 & Pu-238 & $1.50 E-01$ & $6.71 E+03$ \\
\hline 24 & Pu-239 & 1.16E-01 & 1.15E+04 \\
\hline 25 & Pu mix-239/241 & & \\
\hline 26 & $P u-240$ & $6.48 E-02$ & $2.61 E+03$ \\
\hline 27 & Pu-241 & $0.00 E+00$ & $1.04 E+03$ \\
\hline 28 & Pu pure-242 & 3.72E-04 & $1.62 E+01$ \\
\hline 29 & Sr-90 & $5.58 \mathrm{E}+01$ & $8.87 E+00$ \\
\hline 30 & Tc-99 & $1.25 E-16$ & $2.50 E-15$ \\
\hline 31 & Th-232 & $4.21 E-06$ & $3.71 E+\infty 0$ \\
\hline 32 & $\mathrm{TI}-204$ & 8.28E-08 & $9.89 E-12$ \\
\hline 33 & $\mathrm{U}-235$ & $1.82 E-04$ & $1.68 \mathrm{E}-04$ \\
\hline 34 & U-238 & 5.12E-04 & $1.20 \mathrm{E}-02$ \\
\hline 35 & Total & & \\
\hline 36 & $\mathrm{Ba}-137 \mathrm{~m}$ & $4.46 E-04$ & \\
\hline 37 & $Y-90$ & $3.55 E+00$ & \\
\hline 38 & Pr-144 & $3.76 \mathrm{E}-07$ & \\
\hline 39 & $\mathrm{~Pa}-233$ & 3.85E-05 & \\
\hline 40 & Th-234 & 9.57E-06 & \\
\hline 41 & $\mathrm{~Pa}-234$ & 2.69E-05 & \\
\hline 42 & $N p-239$ & 7.49E-05 & \\
\hline 43 & Th-231 & $1.35 E-08$ & \\
\hline 44 & Ra-228 & $1.48 \mathrm{E}-12$ & \\
\hline 45 & Ac-228 & 2.76E-07 & \\
\hline 46 & & & \\
\hline 47 & Total & $3.49 E+02$ & $3.19 E+04$ \\
\hline
\end{tabular}




\begin{tabular}{|c|c|c|c|}
\hline & $\mathbf{B}$ & $\bar{K}$ & $L$ \\
\hline 9 & Nuclide & Skin Dose (mrem per uCi/m^2) & BS Dose (mrem per uCl/m^2) \\
\hline 10 & Am-241 & $=\$ C \$ 7^{*} \$ D 10^{*}$ Relocation! $\$ F 10^{*} \$ C \$ 35 / \$ E \$ 35^{*} Q 10$ & $=\$ C \$ 7^{*} \$ C \$ 3^{*} \$ C \$ 4^{*} \$ C \$ 5^{*} \$ C \$ 6^{*} \$ F 10^{*}$ Relocation! $\$ 10^{*} Q 10$ \\
\hline 11 & Am-243 & $=\$ C \$ 7^{*} \$ D 11^{*}$ Relocation!\$F11*\$C\$35/\$E $\$ 35^{*} Q 11$ & $=\$ C \$ 7^{*} \$ C \$ 3^{*} \$ C \$ 4^{*} \$ C \$ 5^{*} \$ C \$ 6^{*} \$ F 11^{*}$ Relocation! $\$ 11^{*} Q 11$ \\
\hline$\overline{12}$ & Ba-133 & $=\$ C \$ 7^{*} \$ D 12^{*}$ Relocation!\$F $12^{*} \$ C \$ 35 / \$ E \$ 35^{*} Q 12$ & $=\$ C \$ 7^{*} \$ C \$ 3^{*} \$ C \$ 4^{*} \$ C \$ 5^{*} \$ C \$ 6^{*} \$ F 12^{*}$ Relocation! $\$ 12^{*} Q 12$ \\
\hline 13 & Ce-144 & $=\$ C \$ 7^{*} \$ D 13^{*}$ Relocation!\$F $13^{*} \$ C \$ 35 / \$ E \$ 35^{*} Q 13$ & $=\$ C \$ 7^{*} \$ C \$ 3^{*} \$ C \$ 4^{*} \$ C \$ 5^{*} \$ C \$ 6^{*} \$ F 13^{*}$ Relocation! $\$ 13^{*} Q 13$ \\
\hline 14 & Cf-249 & $=\$ C \$ 7^{*} \$ D 14^{*}$ Relocation!\$F14*\$C\$35/\$E\$35*Q14 & $=\$ C \$ 7^{*} \$ C \$ 3^{*} \$ C \$ 4^{*} \$ C \$ 5^{*} \$ C \$ 6^{*} \$ F 14^{*}$ Relocation! $\$ 14^{*} Q 14$ \\
\hline 15 & CF-252 & $=\$ C \$ 7^{*} \$ D 15^{*}$ Relocation!\$F15*\$C\$35/\$E\$35*Q15 & $=\$ C \$ 7^{*} \$ C \$ 3^{*} \$ C \$ 4^{*} \$ C \$ 5^{*} \$ C \$ 6^{*} \$ F 15^{*}$ Relocation!\$15*Q15 \\
\hline 16 & $\mathrm{Cm}-244$ & $=\$ C \$ 7^{*} \$ D 16^{*}$ Relocation! $\$ F 16^{*} \$ C \$ 35 / \$ E \$ 35^{*} Q 16$ & $=\$ C \$ 7^{*} \$ C \$ 3^{*} \$ C \$ 4^{*} \$ C \$ 5^{*} \$ C \$ 6^{*} \$ F 16^{*}$ Relocation! $\$ 16^{*} Q 16$ \\
\hline 17 & $\mathrm{Cm}-246$ & $=\$ C \$ 7^{*} \$ D 17^{*}$ Relocation!\$F $17^{*} \$ C \$ 35 / \$ E \$ 35^{*} Q 17$ & $=\$ C \$ 7^{*} \$ C \$ 3^{*} \$ C \$ 4^{*} \$ C \$ 5^{*} \$ C \$ 6^{*} \$ F 17^{*}$ Relocation! $\$ 17^{*} Q 17$ \\
\hline 18 & Co-60 & $=\$ C \$ 7^{*} \$ D 18^{*}$ Relocation!\$F18*\$C\$35/\$E\$35*Q18 & $=\$ C \$ 7^{*} \$ C \$ 3^{*} \$ C \$ 4^{*} \$ C \$ 5^{*} \$ C \$ 6^{*} \$ F 18^{*}$ Relocation! $\$ 18^{*} Q 18$ \\
\hline 19 & Cs-137 & $=\$ C \$ 7^{*} \$ D 19^{*}$ Relocation!\$F $19^{*} \$ C \$ 35 / \$ E \$ 35^{*} Q 19$ & $=\$ C \$ 7^{*} \$ C \$ 3^{*} \$ C \$ 4^{*} \$ C \$ 5^{*} \$ C \$ 6^{*} \$ F 19^{*}$ Relocation!\$J19*Q19 \\
\hline 20 & $\mathrm{H}-3$ & $=\$ C \$ 7^{*} \$ D 20^{*}$ Relocation!\$F20*\$C\$35/\$E\$35*Q20 & $=\$ C \$ 7^{*} \$ C \$ 3^{*} \$ C \$ 4^{*} \$ C \$ 5^{*} \$ C \$ 6^{*} \$ F 20^{*}$ Relocation!\$J20*Q20 \\
\hline 21 & Np-237 & $=\$ C \$ 7^{*} \$ D 21^{*}$ Relocation!\$F21*\$C\$35/\$E $\$ 35^{*} Q 21$ & $=\$ C \$ 7^{*} \$ C \$ 3^{*} \$ C \$ 4^{*} \$ C \$ 5^{*} \$ C \$ 6^{*} \$ 21^{*}$ Relocation! $\$ 21^{*} \mathrm{Q} 21$ \\
\hline 22 & $P m-147$ & $=\$ C \$ 7^{*} \$ D 22^{*}$ Relocation!\$F22*\$C\$35/\$E\$35*Q22 & $=\$ C \$ 7^{*} \$ C \$ 3^{*} \$ C \$ 4^{*} \$ C \$ 5^{*} \$ C \$ 6^{*} \$ F 22^{*}$ Relocation! $\$ J 22^{*} Q 22$ \\
\hline 23 & Pu-238 & $=\$ C \$ 7^{*} \$ D 23^{*}$ Relocation!\$F23*\$C $\$ 35 / \$ E \$ 35^{*} Q 23$ & $=\$ C \$ 7^{*} \$ C \$ 3^{*} \$ C \$ 4^{*} \$ C \$ 5^{*} \$ C \$ 6^{*} \$ F 23^{*}$ Relocation $\mid \$ \mathrm{~J}^{2} 3^{*} \mathrm{Q} 23$ \\
\hline 24 & Pu-239 & $=\$ C \$ 7^{*} \$ D 24^{*}$ Relocation! $\$ F 24^{*} \$ C \$ 35 / \$ E \$ 35^{*} Q 24$ & $=\$ C \$ 7^{*} \$ C \$ 3^{*} \$ C \$ 4^{*} \$ C \$ 5^{*} \$ C \$ 6^{*} \$ F 24^{*}$ Relocation! $\$ 24^{*} Q 24$ \\
\hline 25 & Pu mix-239/241 & & \\
\hline 26 & Pu-240 & $=\$ C \$ 7^{*} \$ D 26^{*}$ Relocation!\$F26*\$C $\$ 35 / \$ E \$ 35^{*} Q 26$ & $=\$ C \$ 7^{*} \$ C \$ 3^{*} \$ C \$ 4^{*} \$ C \$ 5^{*} \$ C \$ 6^{*} \$ 26^{*}$ Relocation! $\$ 26^{*} Q 26$ \\
\hline 27 & Pu-241 & $=\$ C \$ 7^{*} \$ D 27^{*}$ Relocation!\$F $27^{*} \$ C \$ 35 / \$ E \$ 35^{*} Q 27$ & $=\$ C \$ 7^{*} \$ C \$ 3^{*} \$ C \$ 4^{*} \$ C \$ 5^{*} \$ C \$ 6^{*} \$ 27^{*}$ Relocation! $\$ \mathrm{~J}^{2} 7^{*} \mathrm{Q} 27$ \\
\hline 28 & Pu pure-242 & $=\$ C \$ 7^{*} \$ D 28^{*}$ Relocation $1 \$ F 28 * \$ C \$ 35 / \$ E \$ 35^{*} Q 28$ & $=\$ C \$ 7^{*} \$ C \$ 3^{*} \$ C \$ 4^{*} \$ C \$ 5^{*} \$ C \$ 6^{*} \$ F 28^{*}$ Relocation $! \$ J^{2} 8^{*} Q 28$ \\
\hline 29 & Sr-90 & $=\$ C \$ 7^{\star} \$ D 29^{*}$ Relocation!\$F29*\$C $\$ 35 / \$ E \$ 35^{*} Q 29$ & $=\$ C \$ 7^{*} \$ C \$ 3^{*} \$ C \$ 4^{*} \$ C \$ 5^{*} \$ C \$ 6^{*} \$ F 29^{*}$ Relocation! $\$ 22^{*} Q 29$ \\
\hline 30 & Tc-99 & $=\$ C \$ 7^{*} \$ D 30^{*}$ Relocation!\$F30*\$C\$35/\$E\$35*Q30 & $=\$ C \$ 7^{*} \$ C \$ 3^{*} \$ C \$ 4^{*} \$ C \$ 5^{*} \$ C \$ 6^{*} \$ F 30^{*}$ Relocation! $\$ 30^{*} Q 30$ \\
\hline$\overline{31}$ & Th-232 & $=\$ C \$ 7 * \$ D 31 *$ Relocation!\$F31"\$C $\$ 35 / \$ E \$ 35^{*} Q 31$ & $=\$ C \$ 7^{*} \$ C \$ 3^{*} \$ C \$ 4^{*} \$ C \$ 5^{*} \$ C \$ 6^{*} \$ F 31^{*}$ Relocation! $\$ J_{31 *}^{*} Q 31$ \\
\hline 32 & TI-204 & $=\$ C \$ 7^{\star} \$ D 32^{\star}$ Relocation!\$F32*\$C\$35/\$E\$35*Q32 & $=\$ C \$ 7^{*} \$ C \$ 3^{*} \$ C \$ 4^{*} \$ C \$ 5^{*} \$ C \$ 6^{*} \$ F 32^{*}$ Relocation!\$J32*Q32 \\
\hline$\overline{33}$ & U-235 & $=\$ C \$ 7^{*} \$ D 33^{*}$ Relocation!\$F33*\$C $\$ 35 / \$ E \$ 35^{*} Q 33$ & $=\$ C \$ 7^{*} \$ C \$ 3^{*} \$ C \$ 4^{*} \$ C \$ 5^{*} \$ C \$ 6^{*} \$ F 33^{*}$ Relocation $\mid \$ J 33^{*} Q 33$ \\
\hline 34 & U-238 & $=\$ C \$ 7^{\star} \$ D 34^{*}$ Relocation!\$F34*\$C $\$ 35 / \$ E \$ 35^{*} Q 34$ & $=\$ C \$ 7^{*} \$ C \$ 3^{*} \$ C \$ 4^{*} \$ C \$ 5^{*} \$ C \$ 6^{*} \$ F 34^{*}$ Relocation! $\$ 34^{*} Q 34$ \\
\hline 35 & Total & & \\
\hline 36 & $\mathrm{Ba}-137 \mathrm{~m}$ & $=\$ C \$ 7^{*} \$ D 36^{*}$ Relocation $1 \$ F 36 * \$ C \$ 35 / \$ E \$ 35^{*} Q 36$ & \\
\hline 37 & Y-90 & $=\$ C \$ 7^{*} \$ D 37^{*}$ Relocation!\$F37 $\$ C \$ 35 / \$ E \$ 35^{*} Q 37$ & \\
\hline 38 & Pr-144 & $=\$ C \$ 7^{*} \$ 038^{*}$ Relocation!\$F38*\$C\$35/\$E\$35*Q38 & \\
\hline 39 & Pa-233 & $=\$ C \$ 7^{*} \$ D 39 *$ Relocation!\$F39*\$C $\$ 35 / \$ E \$ 35^{*} Q 39$ & \\
\hline 40 & Th-234 & $=\$ C \$ 7^{*} \$ D 40^{*}$ Relocation! $\$ 440 * \$ C \$ 35 / \$ E \$ 35^{*} Q 40$ & \\
\hline$\overline{41}$ & Pa-234 & $=\$ C \$ 7^{*} \$ D 41^{*}$ Relocation!\$F41*\$C $\$ 35 / \$ E \$ 35^{*} Q 41$ & \\
\hline$\overline{42}$ & Np-239 & $=\$ C \$ 7^{*} \$ D 42^{*}$ Relocation!\$F42*\$C $\$ 35 / \$ E \$ 35^{*} Q 42$ & \\
\hline 43 & Th-231 & $=\$ C \$ 7^{*} \$ D 43^{*}$ Relocation! $\$ 43^{*} \$ C \$ 35 / \$ E \$ 35^{*} Q 43$ & \\
\hline 44 & Ra-228 & $=\$ C \$ 7^{*} \$ D 44^{*}$ Relocation $\mid \$ F 44^{*} \$ C \$ 35 / \$ E \$ 35^{*} Q 44$ & \\
\hline$\overline{45}$ & AC-228 & $=\$ C \$ 7^{*} \$ D 45^{*}$ Relocation!\$F45*\$C $\$ 35 / \$ E \$ 35^{*} Q 45$ & \\
\hline 46 & & & \\
\hline 47 & Total & $=S U M(K 10: K 46)$ & $=S U M(L 10: L 46)$ \\
\hline
\end{tabular}




\begin{tabular}{|c|c|c|c|}
\hline & $\mathrm{B}$ & $\bar{M}$ & $\mathbf{N}$ \\
\hline 9 & Nuclide & $\begin{array}{l}\text { Halflife } \\
\text { (years) }\end{array}$ & Lambda1 \\
\hline 10 & Am-241 & 458 & 0.0015131 \\
\hline 11 & Am-243 & 7950 & 8.71698E-05 \\
\hline 12 & Ba-133 & 10.5 & 0.066 \\
\hline 13 & $\mathrm{Ce}-144$ & 0.7780822 & 0.890651408 \\
\hline 14 & Cf-249 & 360 & 0.001925 \\
\hline 15 & Cf-252 & 2.646 & 0.261904762 \\
\hline 16 & $\mathrm{Cm}-244$ & 17.6 & 0.039375 \\
\hline 17 & $\mathrm{Cm}-246$ & 5500 & 0.000126 \\
\hline 18 & Co-60 & 5.26 & 0.131749049 \\
\hline 19 & Cs-137 & 30 & 0.0231 \\
\hline 20 & $\mathrm{H}-3$ & 12.3 & 0.056341463 \\
\hline 21 & $\mathrm{~Np}-237$ & 2140000 & 3.23832E-07 \\
\hline 22 & Pm-147 & 2.62 & 0.264503817 \\
\hline 23 & Pu-238 & 86.4 & 0.008020833 \\
\hline 24 & Pu-239 & 24390 & 2.84133E-05 \\
\hline 25 & Pu mix-239/241 & & \\
\hline 26 & Pu-240 & 6580 & 0.000105319 \\
\hline 27 & Pu-241 & 13.2 & 0.0525 \\
\hline 28 & Pu pure-242 & 379000 & 1.8285E-06 \\
\hline 29 & Sr-90 & 28.1 & 0.024661922 \\
\hline 30 & TC-99 & 212000 & $3.26887 E-06$ \\
\hline 31 & Th-232 & $1.4 E+10$ & $\quad 4.95 \mathrm{E}-11$ \\
\hline 32 & TI-204 & 3.8 & 0.182368421 \\
\hline 33 & $\mathrm{U}-235$ & 710000000 & $9.76056 \mathrm{E}-10$ \\
\hline 34 & U-238 & 4.51E+09 & $1.53659 E-10$ \\
\hline 35 & Total & & \\
\hline 36 & $\mathrm{Ba}-137 \mathrm{~m}$ & 4:855E-06 & 142727.5862 \\
\hline 37 & $Y-90$ & 0.0073174 & 94.70639626 \\
\hline 38 & Pr-144 & 3.288E-05 & 21078.75 \\
\hline 39 & $\mathrm{~Pa}-233$ & 0.0739726 & 9.368333333 \\
\hline 40 & Th-234 & 0.0660274 & 10.49564315 \\
\hline 41 & $\mathrm{~Pa}-234$ & 0.0007648 & 906.0716418 \\
\hline 42 & Np-239 & 0.0064521 & 107.4076433 \\
\hline 43 & Th-231 & 0.0029132 & 237.8793103 \\
\hline 44 & $\mathrm{Ra}-228$ & 5.75 & 0.120521739 \\
\hline 45 & $A C-228$ & 0.0006998 & 990.3230016 \\
\hline
\end{tabular}




\begin{tabular}{|c|c|c|c|}
\hline & B & $\bar{M}$ & $\mathbf{N}$ \\
\hline 9 & Nuclide & Halflife (years) & Lambda1 \\
\hline 10 & Am-241 & $=T$ _half!C2 & $=0.693 / \mathrm{M} 10$ \\
\hline 11 & Am-243 & $=T$ half!C3 & $=0.693 / \mathrm{M} 11$ \\
\hline 12 & $\mathrm{Ba}-133$ & $=T$ _half!C4 & $=0.693 / \mathrm{M} 12$ \\
\hline 13 & Ce-144 & $=T$ _half!C5 & $=0.693 / \mathrm{M} 13$ \\
\hline 14 & $C f-249$ & $=T_{-}^{-}$half!C6 & $=0.693 / \mathrm{M} 14$ \\
\hline 15 & Cf-252 & $=T$ half!C7 & $=0.693 / \mathrm{M} 15$ \\
\hline 16 & $\mathrm{Cm}-244$ & $=T$ half!C8 & $=0.693 / \mathrm{M} 16$ \\
\hline 17 & $\mathrm{Cm}-246$ & $=T$ half $! C 9$ & $=0.693 / \mathrm{M} 17$ \\
\hline 18 & Co-60 & $=T$ halfiC 10 & $=0.693 / \mathrm{M} 18$ \\
\hline 19 & Cs-137 & $=T$ half!C11 & $=0.693 / \mathrm{M} 19$ \\
\hline 20 & $\mathrm{H}-3$ & $=T$ halflC12 & $=0.693 / \mathrm{M} 20$ \\
\hline 21 & Np-237 & $=T$ half!C13 & $=0.693 / \mathrm{M} 21$ \\
\hline 22 & Pm-147 & $=T$ half!C14 & $=0.693 / \mathrm{M} 22$ \\
\hline 23 & Pu-238 & $=T$ halflC15 & $=0.693 / \mathrm{M} 23$ \\
\hline 24 & Pu-239 & $=T$ half!C16 & $=0.693 / \mathrm{M} 24$ \\
\hline 25 & Pu mix-239/241 & & \\
\hline 26 & Pu-240 & $=T$ haif!C18 & $=0.693 / \mathrm{M} 26$ \\
\hline 27 & Pu-241 & $=T$ half!C19 & $=0.693 / \mathrm{M} 27$ \\
\hline 28 & Pu pure-242 & $=T$ haiflC20 & $=0.693 / \mathrm{M} 28$ \\
\hline 29 & Sr-90 & $=T$ half!C21 & $=0.693 / \mathrm{M} 29$ \\
\hline 30 & TC-99 & $=T$ half!C22 & $=0.693 / \mathrm{M} 30$ \\
\hline 31 & Th-232 & $=T$ half!C23 & $=0.693 / \mathrm{M3} 1$ \\
\hline 32 & TI-204 & $=T$ halfiC24 & $=0.693 / \mathrm{M} 32$ \\
\hline 33 & U-235 & $=T$ half!C25 & $=0.693 / \mathrm{M} 33$ \\
\hline 34 & U-238 & $=T$ half!C26 & $=0.693 / \mathrm{M} 34$ \\
\hline 35 & Total & & \\
\hline 36 & $B a-137 m$ & =T_half!C28 & $=0.693 / \mathrm{M} 36$ \\
\hline 37 & $Y-90$ & $=T$ half!C29 & $=0.693 / \mathrm{M} 37$ \\
\hline 38 & Pr-144 & $=T$ half!C30 & $=0.693 / \mathrm{M} 38$ \\
\hline 39 & $\mathrm{~Pa}-233$ & $=T_{\text {_halfl }}$ C31 & $=0.693 / \mathrm{M} 39$ \\
\hline 40 & Th-234 & $=T$ half!C32 & $=0.693 / \mathrm{M} 40$ \\
\hline 41 & $\mathrm{~Pa}-234$ & $=T_{\text {_half! }}$ C33 & $=0.693 / \mathrm{M} 41$ \\
\hline 42 & $N p-239$ & $=T$ half!C34 & $=0.693 / \mathrm{M} 42$ \\
\hline 43 & Th-231 & $=T$ half!C35 & $=0.693 / \mathrm{M} 43$ \\
\hline 44 & $\mathrm{Ra}-228$ & $=T$ half!C36 & $=0.693 / \mathrm{M} 44$ \\
\hline 45 & $A c-228$ & $=T$ half!C37 & $=0.693 / \mathrm{M} 45$ \\
\hline
\end{tabular}




\begin{tabular}{|c|c|c|}
\hline & B & $\bar{Q}$ \\
\hline 9 & Nuclide & $\begin{array}{l}\text { Weathering Factor } \\
\text { ( } 50 \text { years) }\end{array}$ \\
\hline 10 & Am-241 & $1.546 E+01$ \\
\hline 11 & Am-243 & $1.596 \mathrm{E}+01$ \\
\hline 12 & Ba-133 & $5.434 E+00$ \\
\hline 13 & $\mathrm{Ce}-144$ & 7.237E-01 \\
\hline 14 & Cf-249 & $1.532 E+01$ \\
\hline 15 & Cf-252 & $1.826 E+00$ \\
\hline 16 & Cm-244 & 7.677E+00 \\
\hline 17 & $\mathrm{Cm}-246$ & $1.595 E+01$ \\
\hline 18 & $\mathrm{Co}-60$ & $3.154 \mathrm{E}+00$ \\
\hline 19 & Cs-137 & $1.002 E+01$ \\
\hline 20 & $\mathrm{H}-3$ & $6.090 E+00$ \\
\hline 21 & Np-237 & $1.599 E+01$ \\
\hline 22 & Pm-147 & 1.812E+00 \\
\hline 23 & Pu-238 & $1.343 E+01$ \\
\hline 24 & Pu-239 & $1.598 E+01$ \\
\hline 25 & Pu mix-239/241 & \\
\hline 26 & $\mathrm{Pu}-240$ & $1.595 E+01$ \\
\hline 27 & Pu-241 & $6.394 E+00$ \\
\hline 28 & Pu pure-242 & $1.599 E+01$ \\
\hline 29 & Sr-90 & $9.749 \mathrm{E}+00$ \\
\hline 30 & Tc-99 & $1.599 E+01$ \\
\hline 31 & Th-232 & $1.599 E+01$ \\
\hline 32 & $\mathrm{Tl}-204$ & $2.429 E+00$ \\
\hline 33 & $\mathrm{U}-235$ & $1.599 E+01$ \\
\hline 34 & U-238 & 1.599E+01 \\
\hline 35 & Total & \\
\hline 36 & Ba-137m & 7.006E-06 \\
\hline 37 & $\gamma-90$ & $1.048 E-02$ \\
\hline 38 & Pr-144 & 4.744E-05 \\
\hline 39 & $\mathrm{~Pa}-233$ & 9.947E-02 \\
\hline 40 & Th-234 & 8.942E-02 \\
\hline 41 & $\mathrm{~Pa}-234$ & $1.103 E-03$ \\
\hline 42 & Np-239 & $9.249 \mathrm{E}-03$ \\
\hline 43 & Th-231 & 4.191E-03 \\
\hline 44 & Ra-228 & $3.390 E+00$ \\
\hline 45 & Ac-228 & $1.009 E-03$ \\
\hline
\end{tabular}




\begin{tabular}{|c|c|c|}
\hline & B & $\bar{Q}$ \\
\hline 9 & Nuclide & Weathering Factor (50 years) \\
\hline 10 & Am-241 & $=\left(0.63 /(\$ N 10+\$ 1 \$ 4)^{*}\left(1-E X P\left(-(\$ N 10+\$ L \$ 4)^{*} \$ P \$ 5\right)\right)\right)+\left((0.37 /(\$ N 10+\$ 1 \$ 6))^{*}\left(1-E X P\left(-(\$ N 10+\$ L \$ 6)^{*} \$ P \$ 5\right)\right)\right)$ \\
\hline 11 & Am-243 & $=\left(0.63 /(\$ N 11+\$ L \$ 4)^{*}\left(1-\operatorname{EXP}\left(-(\$ N 11+\$ L \$ 4)^{*} \$ P \$ 5\right)\right)\right)+\left((0.37 /(\$ N 11+\$ L \$ 6))^{*}\left(1-\operatorname{EXP}\left(-(\$ N 11+\$ L \$ 6)^{*} \$ P \$ 5\right)\right)\right)$ \\
\hline 12 & Ba-133 & $=\left(0.63 /(\$ N 12+\$ L \$ 4)^{\star}\left(1-\operatorname{EXP}\left(-(\$ N 12+\$ L \$ 4)^{\star} \$ P \$ 5\right)\right)\right)+\left((0.37 /(\$ N 12+\$ L \$ 6))^{*}\left(1-\operatorname{EXP}\left(-(\$ N 12+\$ L \$ 6)^{\star} \$ P \$ 5\right)\right)\right)$ \\
\hline 13 & Ce-144 & $=\left(0.63 /(\$ N 13+\$ L \$ 4)^{*}\left(1-\operatorname{EXP}\left(-(\$ N 13+\$ L \$ 4)^{*} \$ P \$ 5\right)\right)\right)+\left((0.37 /(\$ N 13+\$ L \$ 6))^{*}\left(1-\operatorname{EXP}\left(-(\$ N 13+\$ L \$ 6)^{*} \$ P \$ 5\right)\right)\right)$ \\
\hline 14 & Cf-249 & $=\left(0.63 /(\$ N 14+\$ L \$ 4)^{*}\left(1-\operatorname{EXP}\left(-(\$ N 14+\$ L \$ 4)^{*} \$ P \$ 5\right)\right)\right)+\left((0.37 /(\$ N 14+\$ L \$ 6))^{*}\left(1-E X P\left(-(\$ N 14+\$ L \$ 6)^{*} \$ P \$ 5\right)\right)\right)$ \\
\hline 15 & Cf-252 & $=\left(0.63 /(\$ N 15+\$ L \$ 4)^{*}\left(1-\operatorname{EXP}\left(-(\$ N 15+\$ L \$ 4)^{\star} \$ P \$ 5\right)\right)\right)+\left((0.37 /(\$ N 15+\$ L \$ 6))^{*}\left(1-E X P\left(-(\$ N 15+\$ L \$ 6)^{*} \$ P \$ 5\right)\right)\right)$ \\
\hline 16 & $\mathrm{Cm}-244$ & $=\left(0.63 /(\$ N 16+\$ L \$ 4)^{*}\left(1-\operatorname{EXP}\left(-(\$ N 16+\$ L \$ 4)^{*} \$ P \$ 5\right)\right)\right)+\left((0.37 /(\$ N 16+\$ L \$ 6))^{*}\left(1-E X P\left(-(\$ N 16+\$ L \$ 6)^{*} \$ P \$ 5\right)\right)\right)$ \\
\hline 17 & $\mathrm{Cm}-246$ & $=\left(0.63 /(\$ N 17+\$ L \$ 4)^{*}\left(1-\operatorname{EXP}\left(-(\$ N 17+\$ L \$ 4)^{*} \$ P \$ 5\right)\right)\right)+\left((0.37 /(\$ N 17+\$ L \$ 6))^{*}\left(1-\operatorname{EXP}\left(-(\$ N 17+\$ L \$ 6)^{*} \$ P \$ 5\right)\right)\right)$ \\
\hline 18 & Co-60 & $=\left(0.63 /(\$ N 18+\$ L \$ 4)^{\star}\left(1-\operatorname{EXP}\left(-(\$ N 18+\$ L \$ 4)^{\star} \$ P \$ 5\right)\right)\right)+\left((0.37 /(\$ N 18+\$ L \$ 6))^{*}\left(1-E X P\left(-(\$ N 18+\$ L \$ 6)^{*} \$ P \$ 5\right)\right)\right)$ \\
\hline 19 & Cs-137 & $=\left(0.63 /(\$ N 19+\$ L \$ 4)^{*}\left(1-E X P\left(-(\$ N 19+\$ L \$ 4)^{*} \$ P \$ 5\right)\right)\right)+\left((0.37 /(\$ N 19+\$ L \$ 6))^{*}\left(1-E X P\left(-(\$ N 19+\$ L \$ 6)^{*} \$ P \$ 5\right)\right)\right)$ \\
\hline 20 & $\mathrm{H}-3$ & $=\left(0.63 /(\$ N 20+\$ L \$ 4)^{*}\left(1-E X P\left(-(\$ N 20+\$ L \$ 4)^{\star} \$ P \$ 5\right)\right)\right)+\left((0.37 /(\$ N 20+\$ L \$ 6))^{*}\left(1-E X P\left(-(\$ N 20+\$ L \$ 6)^{\star} \$ P \$ 5\right)\right)\right)$ \\
\hline 21 & Np-237 & $=\left(0.63 /(\$ N 21+\$ L \$ 4)^{*}\left(1-\operatorname{EXP}\left(-(\$ N 21+\$ L \$ 4)^{*} \$ P \$ 5\right)\right)\right)+\left((0.37 /(\$ N 21+\$ L \$ 6))^{*}\left(1-E X P\left(-(\$ N 21+\$ L \$ 6)^{*} \$ P \$ 5\right)\right)\right)$ \\
\hline 22 & Pm-147 & $=\left(0.63 /(\$ N 22+\$ L \$ 4)^{*}\left(1-\operatorname{EXP}\left(-(\$ N 22+\$ L \$ 4)^{*} \$ P \$ 5\right)\right)\right)+\left((0.37 /(\$ N 22+\$ L \$ 6))^{*}\left(1-\operatorname{EXP}\left(-(\$ N 22+\$ L \$ 6)^{*} \$ P \$ 5\right)\right)\right)$ \\
\hline 23 & Pu-238 & $=\left(0.63 /(\$ N 23+\$ L \$ 4)^{*}\left(1-\operatorname{EXP}\left(-(\$ N 23+\$ L \$ 4)^{\star} \$ P \$ 5\right)\right)\right)+\left((0.37 /(\$ N 23+\$ L \$ 6))^{*}\left(1-E X P\left(-(\$ N 23+\$ L \$ 6)^{*} \$ P \$ 5\right)\right)\right)$ \\
\hline 24 & Pu-239 & $=\left(0.63 /(\$ N 24+\$ L \$ 4)^{*}\left(1-E X P\left(-(\$ N 24+\$ L \$ 4)^{*} \$ P \$ 5\right)\right)\right)+\left((0.37 /(\$ N 24+\$ L \$ 6))^{*}\left(1-E X P\left(-(\$ N 24+\$ L \$ 6)^{*} \$ P \$ 5\right)\right)\right)$ \\
\hline 25 & Pu mix-239/241 & \\
\hline 26 & Pu-240 & $=\left(0.63 /(\$ N 26+\$ L \$ 4)^{*}\left(1-E X P\left(-(\$ N 26+\$ L \$ 4)^{\star} \$ P \$ 5\right)\right)\right)+\left((0.37 /(\$ N 26+\$ L \$ 6))^{*}\left(1-E X P\left(-(\$ N 26+\$ L \$ 6)^{\star} \$ P \$ 5\right)\right)\right)$ \\
\hline 27 & $\mathrm{Pu}-241$ & $=\left(0.63 /(\$ N 27+\$ L \$ 4)^{*}\left(1-\operatorname{EXP}\left(-(\$ N 27+\$ L \$ 4)^{*} \$ P \$ 5\right)\right)\right)+\left((0.37 /(\$ N 27+\$ L \$ 6))^{*}\left(1-\operatorname{EXP}\left(-(\$ N 27+\$ L \$ 6)^{*} \$ P \$ 5\right)\right)\right)$ \\
\hline 28 & Pu pure-242 & $=\left(0.63 /(\$ N 28+\$ L \$ 4)^{*}\left(1-\operatorname{EXP}\left(-(\$ N 28+\$ L \$ 4)^{*} \$ P \$ 5\right)\right)\right)+\left((0.37 /(\$ N 28+\$ L \$ 6))^{*}\left(1-E X P\left(-(\$ N 28+\$ L \$ 6)^{*} \$ P \$ 5\right)\right)\right)$ \\
\hline 29 & Sr-90 & $=\left(0.63 /(\$ N 29+\$ L \$ 4)^{*}\left(1-E X P\left(-(\$ N 29+\$ L \$ 4)^{*} \$ P \$ 5\right)\right)\right)+\left((0.37 /(\$ N 29+\$ L \$ 6))^{*}\left(1-E X P\left(-(\$ N 29+\$ L \$ 6)^{*} \$ P \$ 5\right)\right)\right)$ \\
\hline 30 & Tc-99 & $=\left(0.63 /(\$ N 30+\$ L \$ 4)^{*}\left(1-\operatorname{EXP}\left(-(\$ N 30+\$ L \$ 4)^{*} \$ P \$ 5\right)\right)\right)+\left((0.37 /(\$ N 30+\$ L \$ 6))^{*}\left(1-\operatorname{EXP}\left(-(\$ N 30+\$ L \$ 6)^{*} \$ P \$ 5\right)\right)\right)$ \\
\hline 31 & Th-232 & $=\left(0.63 /(\$ N 31+\$ L \$ 4)^{*}\left(1-\operatorname{EXP}\left(-(\$ N 31+\$ L \$ 4)^{*} \$ P \$ 5\right)\right)\right)+\left((0.37 /(\$ N 31+\$ L \$ 6))^{*}\left(1-\operatorname{EXP}\left(-(\$ N 31+\$ L \$ 6)^{*} \$ P \$ 5\right)\right)\right)$ \\
\hline 32 & TI-204 & $=\left(0.63 /(\$ N 32+\$ L \$ 4)^{*}\left(1-\operatorname{EXP}\left(-(\$ N 32+\$ L \$ 4)^{*} \$ P \$ 5\right)\right)\right)+\left((0.37 /(\$ N 32+\$ L \$ 6))^{*}\left(1-\operatorname{EXP}\left(-(\$ N 32+\$ L \$ 6)^{*} \$ P \$ 5\right)\right)\right)$ \\
\hline 33 & U-235 & $=\left(0.63 /(\$ N 33+\$ L \$ 4)^{*}\left(1-\operatorname{EXP}\left(-(\$ N 33+\$ L \$ 4)^{*} \$ P \$ 5\right)\right)\right)+\left((0.37 /(\$ N 33+\$ L . \$ 6))^{*}\left(1-\operatorname{EXP}\left(-(\$ N 33+\$ L \$ 6)^{*} \$ P \$ 5\right)\right)\right)$ \\
\hline 34 & U-238 & $=\left(0.63 /(\$ N 34+\$ L \$ 4)^{*}\left(1-\operatorname{EXP}\left(-(\$ N 34+\$ L \$ 4)^{\star} \$ P \$ 5\right)\right)\right)+\left((0.37 /(\$ N 34+\$ L \$ 6))^{*}\left(1-\operatorname{EXP}\left(-(\$ N 34+\$ L \$ 6)^{*} \$ P \$ 5\right)\right)\right)$ \\
\hline 35 & Total & \\
\hline 36 & Ba-137m & $=\left(0.63 /(\$ N 36+\$ L \$ 4)^{*}\left(1-\operatorname{EXP}\left(-(\$ N 36+\$ L \$ 4)^{*} \$ P \$ 5\right)\right)\right)+\left((0.37 /(\$ N 36+\$ L \$ 6))^{*}\left(1-\operatorname{EXP}\left(-(\$ N 36+\$ L \$ 6)^{*} \$ P \$ 5\right)\right)\right)$ \\
\hline 37 & $Y-90$ & $=\left(0.63 /(\$ N 37+\$ L \$ 4)^{*}\left(1-E X P\left(-(\$ N 37+\$ L \$ 4)^{\star} \$ P \$ 5\right)\right)\right)+\left((0.37 /(\$ N 37+\$ L \$ 6))^{*}\left(1-E X P\left(-(\$ N 37+\$ L \$ 6)^{*} \$ P \$ 5\right)\right)\right)$ \\
\hline 38 & Pr-144 & $=\left(0.63 /(\$ N 38+\$ L \$ 4)^{\star}\left(1-\operatorname{EXP}\left(-(\$ N 38+\$ L \$ 4)^{*} \$ P \$ 5\right)\right)\right)+\left((0.37 /(\$ N 38+\$ L \$ 6))^{*}\left(1-\operatorname{EXP}\left(-(\$ N 38+\$ L \$ 6)^{*} \$ P \$ 5\right)\right)\right)$ \\
\hline 39 & Pa-233 & $=\left(0.63 /(\$ N 39+\$ L \$ 4)^{\star}\left(1-E X P\left(-(\$ N 39+\$ L \$ 4)^{\star} \$ P \$ 5\right)\right)\right)+\left((0.37 /(\$ N 39+\$ L \$ 6))^{*}\left(1-E X P\left(-(\$ N 39+\$ L \$ 6)^{*} \$ P \$ 5\right)\right)\right)$ \\
\hline 40 & Th-234 & $=\left(0.63 /(\$ N 40+\$ L \$ 4)^{\star}\left(1-\operatorname{EXP}\left(-(\$ N 40+\$ L \$ 4)^{*} \$ P \$ 5\right)\right)\right)+\left((0.37 /(\$ N 40+\$ L \$ 6))^{*}\left(1-E X P\left(-(\$ N 40+\$ L \$ 6)^{*} \$ P \$ 5\right)\right)\right)$ \\
\hline 41 & Pa-234 & $=\left(0.63 /(\$ N 41+\$ L \$ 4)^{*}\left(1-\operatorname{EXP}\left(-(\$ N 41+\$ L \$ 4)^{*} \$ P \$ 5\right)\right)\right)+\left((0.37 /(\$ N 41+\$ L \$ 6))^{*}(1-E X P(-(\$ N 41+\$ L \$ 6) * \$ P \$ 5))\right)$ \\
\hline 42 & Np-239 & $=\left(0.63 /(\$ N 42+\$ L \$ 4)^{*}\left(1-E X P\left(-(\$ N 42+\$ L \$ 4)^{*} \$ P \$ 5\right)\right)\right)+\left((0.37 /(\$ N 42+\$ L \$ 6))^{*}\left(1-E X P\left(-(\$ N 42+\$ L \$ 6)^{*} \$ P \$ 5\right)\right)\right)$ \\
\hline 43 & Th-231 & $=\left(0.63 /(\$ N 43+\$ L \$ 4)^{*}\left(1-\operatorname{EXP}\left(-(\$ N 43+\$ L \$ 4)^{\star} \$ P \$ 5\right)\right)\right)+\left((0.37 /(\$ N 43+\$ L \$ 6))^{*}\left(1-\operatorname{EXP}\left(-(\$ N 43+\$ L \$ 6)^{\star} \$ P \$ 5\right)\right)\right)$ \\
\hline 44 & Ra-228 & $=\left(0.63 /(\$ N 44+\$ L \$ 4)^{*}\left(1-\operatorname{EXP}\left(-(\$ N 44+\$ L \$ 4)^{\star} \$ P \$ 5\right)\right)\right)+\left((0.37 /(\$ N 44+\$ L \$ 6))^{*}\left(1-E X P\left(-(\$ N 44+\$ L \$ 6)^{*} \$ P \$ 5\right)\right)\right)$ \\
\hline 45 & Ac-228 & $=\left(0.63 /(\$ N 45+\$ L \$ 4)^{*}\left(1-\operatorname{EXP}\left(-(\$ N 45+\$ L \$ 4)^{*} \$ P \$ 5\right)\right)\right)+\left((0.37 /(\$ N 45+\$ L \$ 6))^{*}\left(1-\operatorname{EXP}\left(-(\$ N 45+\$ L \$ 6)^{*} \$ P \$ 5\right)\right)\right)$ \\
\hline
\end{tabular}




\begin{tabular}{|c|c|c|}
\hline & G & \multicolumn{1}{c|}{ H } \\
\hline 52 & $2.54 E+00$ & DRL (uCi/m^2) - PAG/(CEDE+External) \\
\cline { 1 - 2 } & $1.56 E+00$ & DRL (uCi/m^2) - PAG/(BS Dose + External \\
\hline 54 & $7.16 E+02$ & DRL (uCi/m^2) - PAG/(Skin Dose) \\
\hline
\end{tabular}




\begin{tabular}{|c|c|c|}
\hline & $\mathbf{G}$ & $\mathbf{H}$ \\
\hline 52 & $=G 3 /(H 47+147)$ & $\overline{D R L}($ (UCi/m^2) - PAG/(CEDE+External) \\
\hline 53 & $=G 4 /(L 47+147)$ & DRL (uCi/m^2) - PAG/(BS Dose + External) \\
\hline 54 & $=\mathrm{G} 5 / \mathrm{K} 47$ & DRL (uCi/m^2) - PAG/(Skin Dose) \\
\hline
\end{tabular}




\begin{tabular}{|c|c|c|}
\hline & $B$ & $\overline{\mathrm{C}}$ \\
\hline 1 & Nuclide & Halfifife (years) \\
\hline 2 & Am-241 & 458 \\
\hline 3 & $A m-243$ & 7950 \\
\hline 4 & Ba.133 & 10.5 \\
\hline 5 & Ce-144 & 0.778082192 \\
\hline 6 & Ci-249 & 360 \\
\hline 7 & $\mathrm{Ct}-252$ & 2.646 \\
\hline 8 & $\mathrm{Cm}-244$ & 17.6 \\
\hline 9 & $\mathrm{Cm}-246$ & 5500 \\
\hline 10 & Co-60 & 5.26 \\
\hline$\pi$ & Cs-137 & 30 \\
\hline 12 & $\mathrm{H}-3$ & 12.3 \\
\hline 13 & $\mathrm{~Np}-237$ & 2140000 \\
\hline 14 & $\mathrm{Pm}=147$ & 2.62 \\
\hline 15 & Pu-238 & 86.4 \\
\hline 16 & Pu-239 & 24390 \\
\hline 17 & Pu mix-239/241 & \\
\hline 18 & Pu-240 & 6580 \\
\hline 19 & Pu-241 & 13.2 \\
\hline 20 & Pu pure-242 & 379000 \\
\hline 21 & Sr-90 & 28.1 \\
\hline 22 & TC-99 & 212000 \\
\hline 23 & Th-232 & 14000000000 \\
\hline 24 & T1-204 & 3.8 \\
\hline 25 & $U-235$ & 710000000 \\
\hline 26 & U-238 & 4510000000 \\
\hline 27 & & \\
\hline 28 & $B a-137 m$ & $4.8554 \mathrm{E}-06$ \\
\hline 29 & $\gamma-90$ & 0.007317352 \\
\hline 30 & Pr-144 & 3.28767E-05 \\
\hline 31 & Pa-233 & 0.073972603 \\
\hline 32 & Th-234 & 0.066027397 \\
\hline 33 & $\mathrm{~Pa}-234$ & 0.00076484 \\
\hline 34 & Np-239 & 0.006452055 \\
\hline 35 & Th-231 & 0.002913242 \\
\hline 36 & Ra-228 & 5.75 \\
\hline 37 & $A c-228$ & 0.000699772 \\
\hline
\end{tabular}




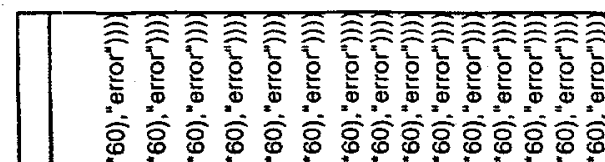

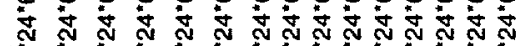
递造造

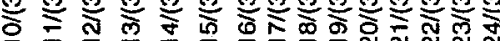

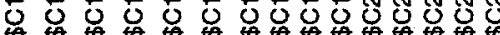

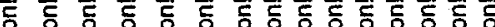
密 要

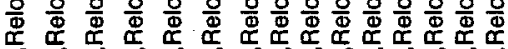
$\bar{E} E \xi \xi \xi \xi \xi \xi \xi \xi \xi$

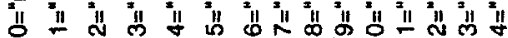

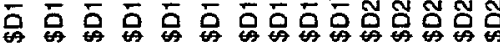
ᄃ ᄃ 要

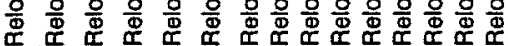

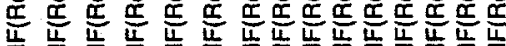

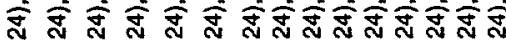
영

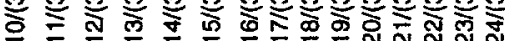
J 亏亏

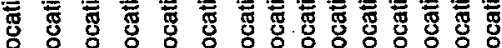

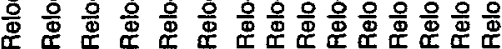

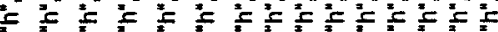

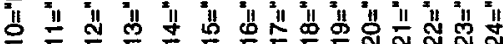

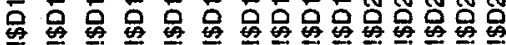
ᄃ

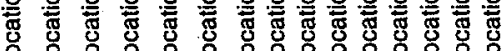

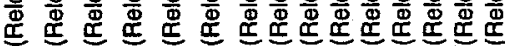

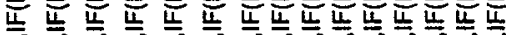

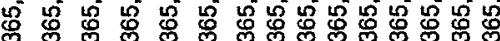
\%응

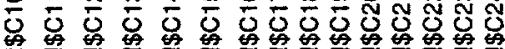

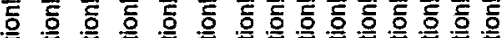

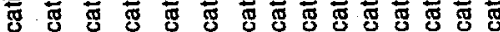

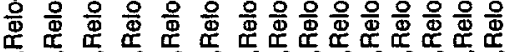

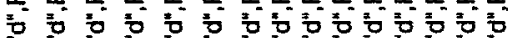

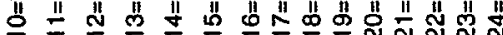

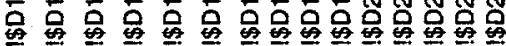

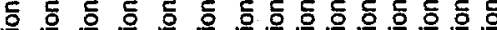
要

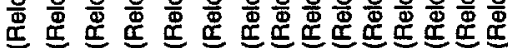

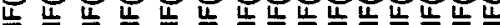
응

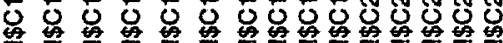

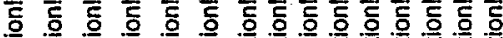

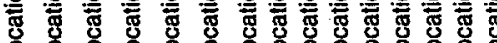

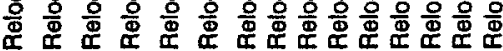
iो il il il il il

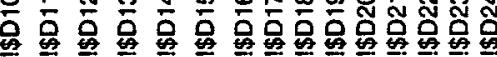

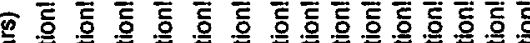
政

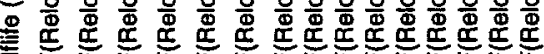

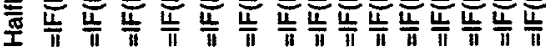

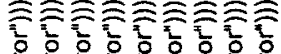

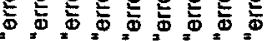

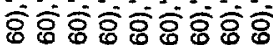

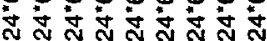

in is in to in

象

के के

S U

들

害

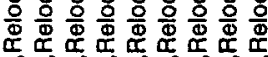

E E E E E E E E E

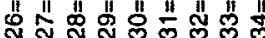

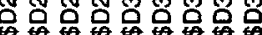

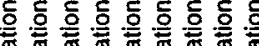

(1)

\% 훙

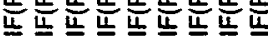

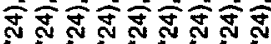

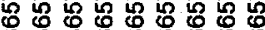

塎

U్

的动

సٓ

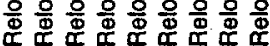

两

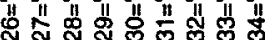

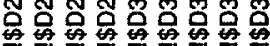

흔

\%

\&

든

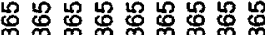

옹

U.

ᄃ 5 ᄃ 5

政 \%

这

$3=050=50$

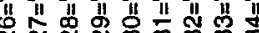

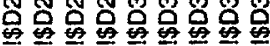

的

\% 8 \%

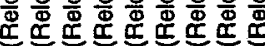

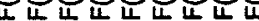

促

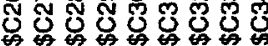

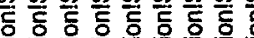

政

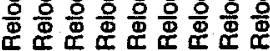

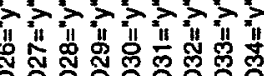

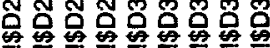

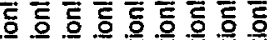

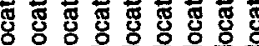

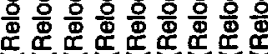

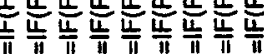

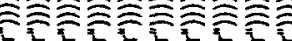

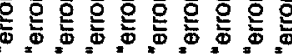

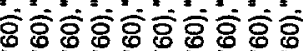

N

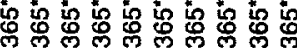

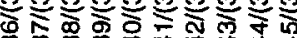

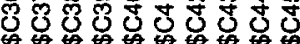

政

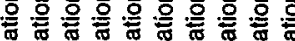

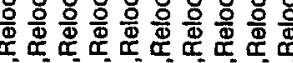

$\xi \xi \xi \xi \xi E \bar{E} E$

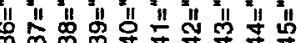

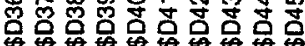

ᄃ

\%

응 응 응 응 응 응 웅 응 응 응

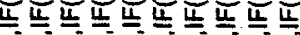

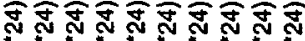

论

क

S

든

覀

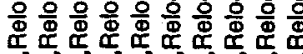

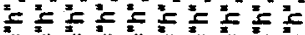

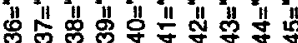

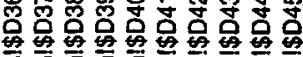

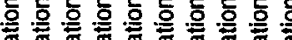

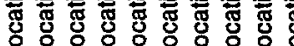

\%

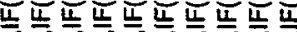

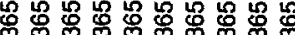

잉

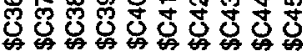

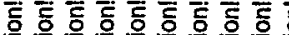

\%

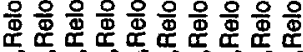

कर

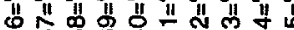

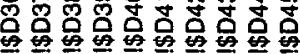

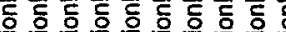

吠

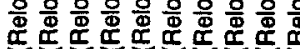

는

of

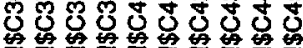

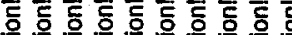

क्ष

क्ष

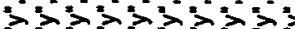

i1

品品品品品

c

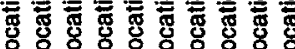

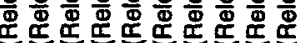

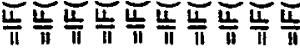
突 离

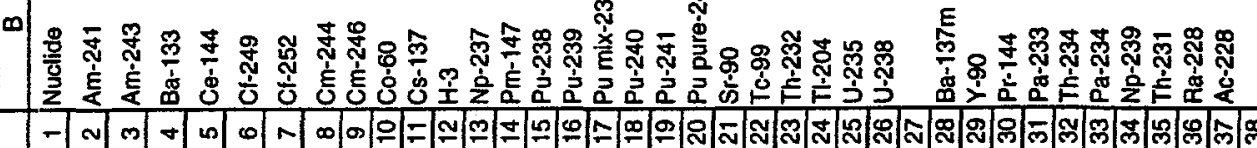




\section{Attachment B}

HOTSPOT results - average meteorology, $0.1 \mathrm{~cm} \mathrm{~s}^{-1}$ 
Footprint plot not available.

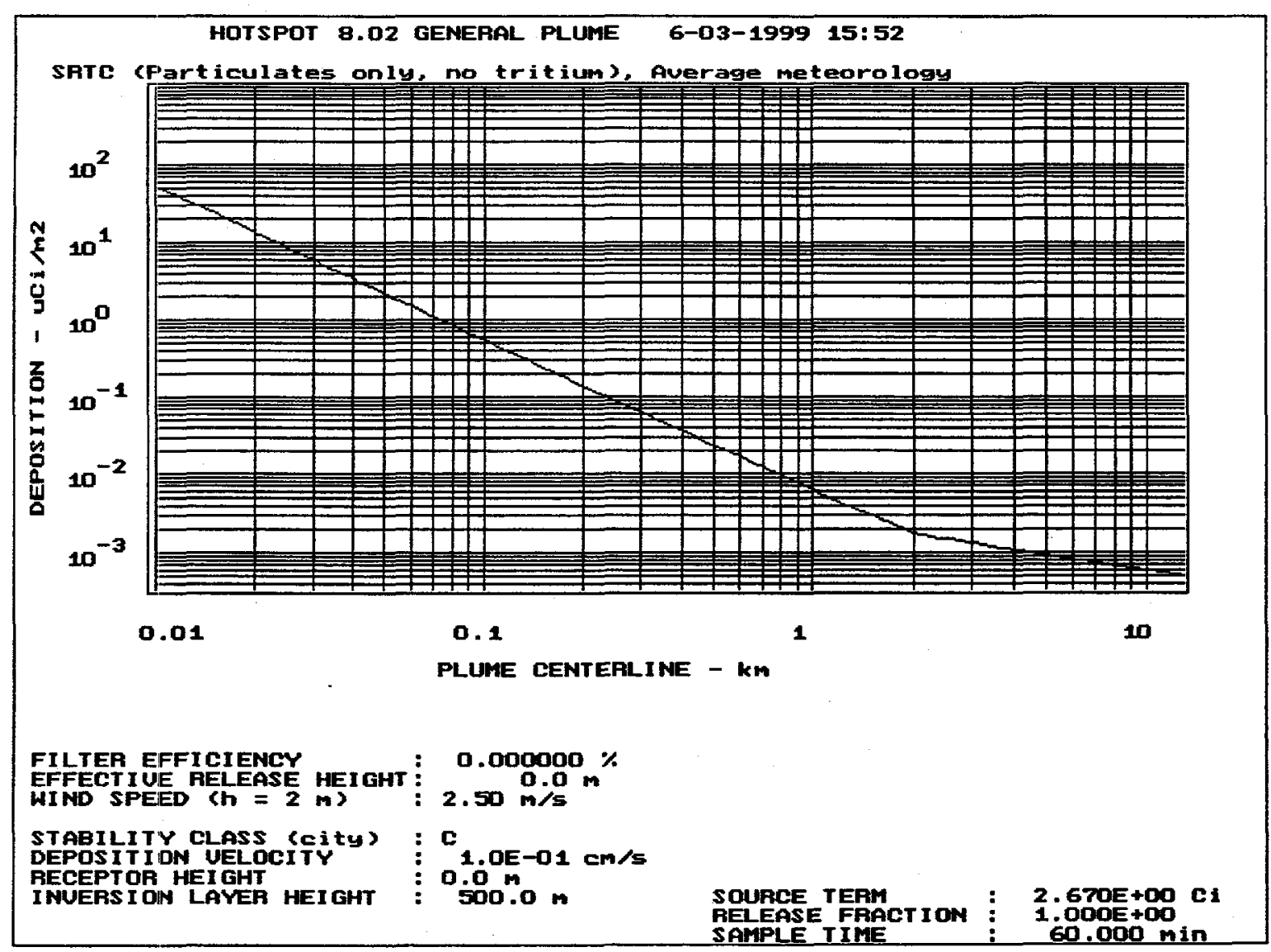




\section{Attachment C}

HOTSPOT results - average meteorology, $1.0 \mathrm{~cm} \mathrm{~s}^{-1}$ 

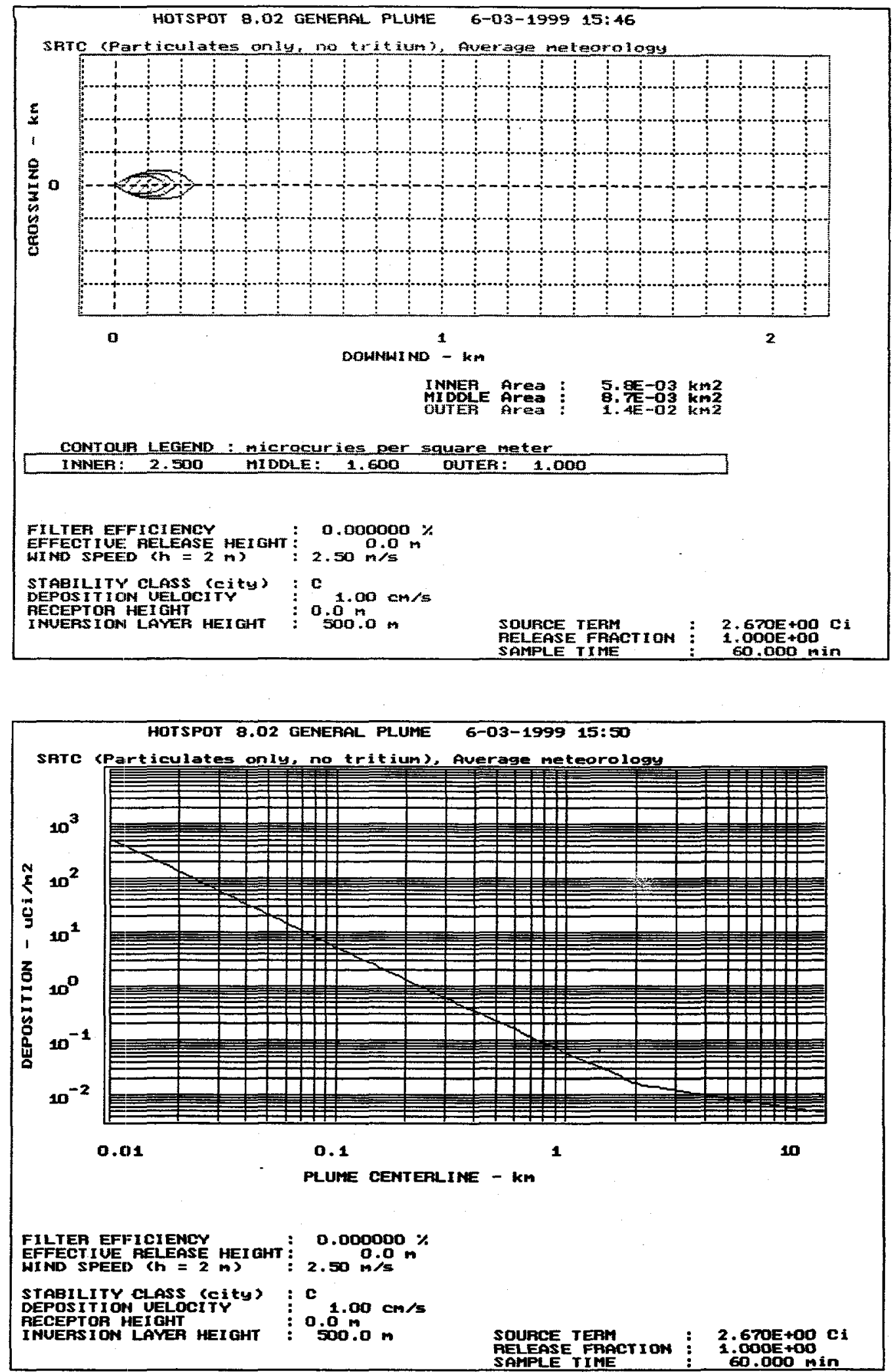


\section{Attachment D}

HOTSPOT results - average meteorology, $10 \mathrm{~cm} \mathrm{~s}^{-1}$ 

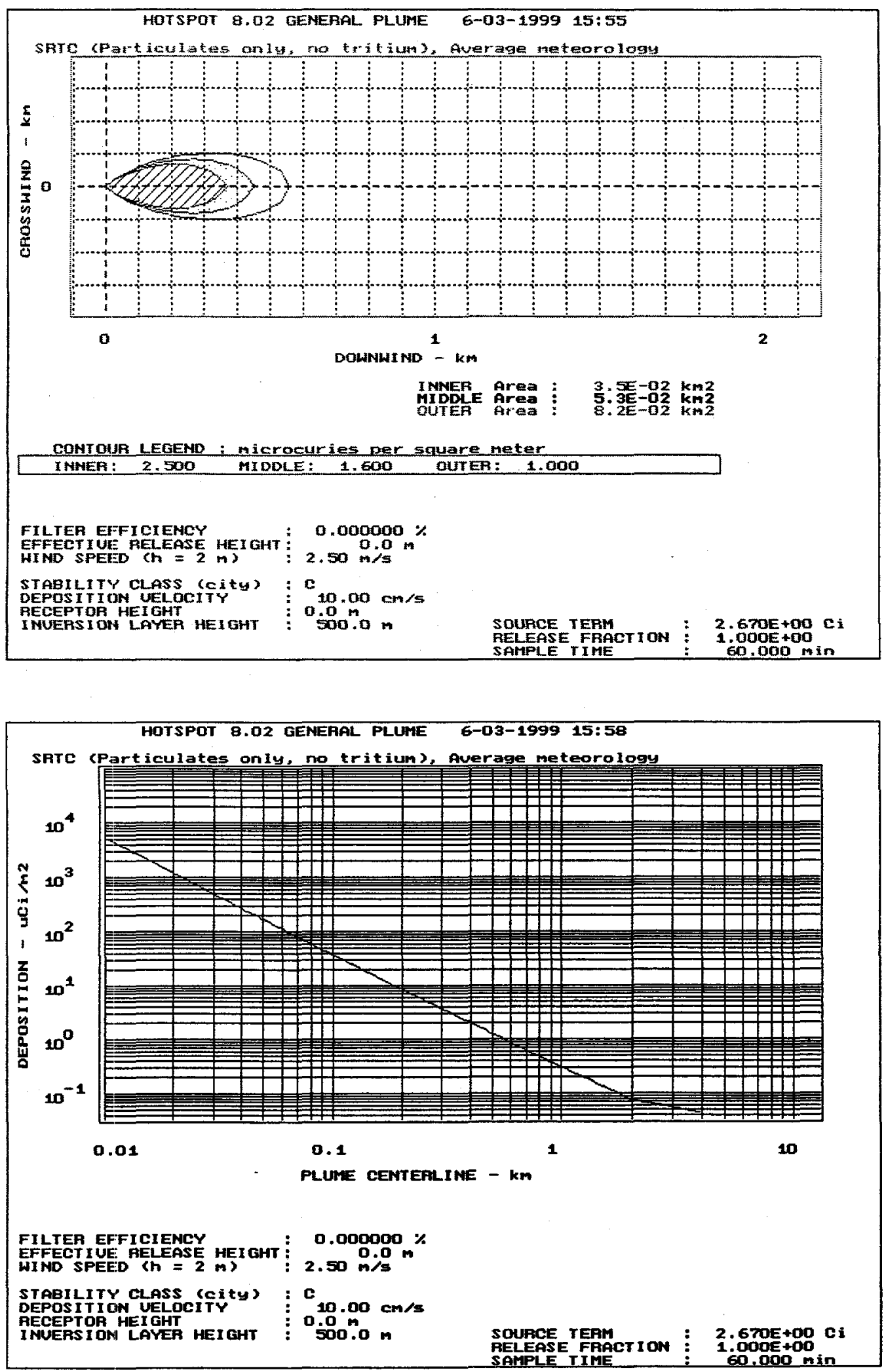


\section{Attachment E}

HOTSPOT results - adverse meteorology, $0.1 \mathrm{~cm} \mathrm{~s}^{-1}$ 

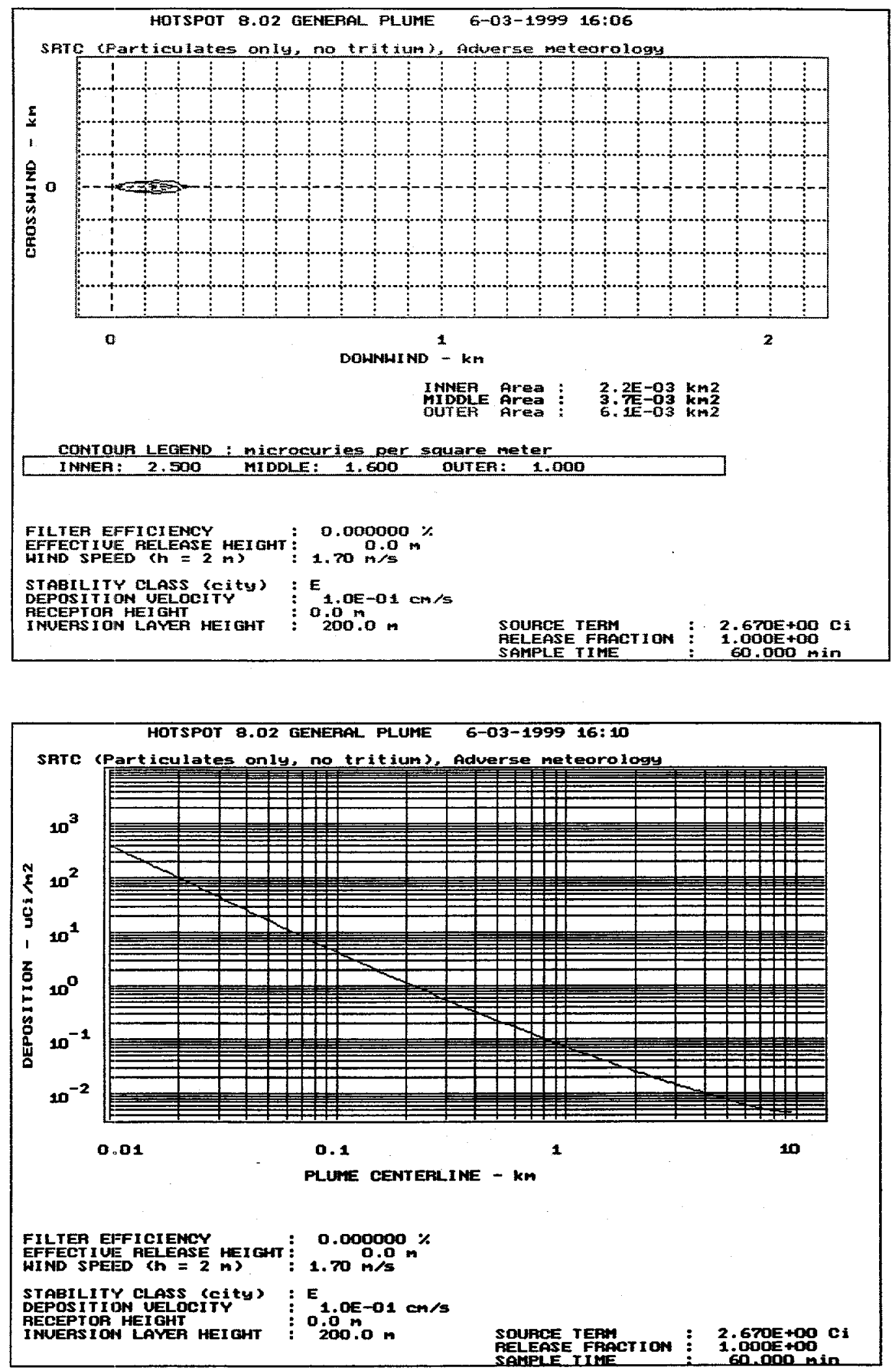


\section{Attachment F}

HOTSPOT results - adverse meteorology, $1.0 \mathrm{~cm} \mathrm{~s}^{-1}$ 

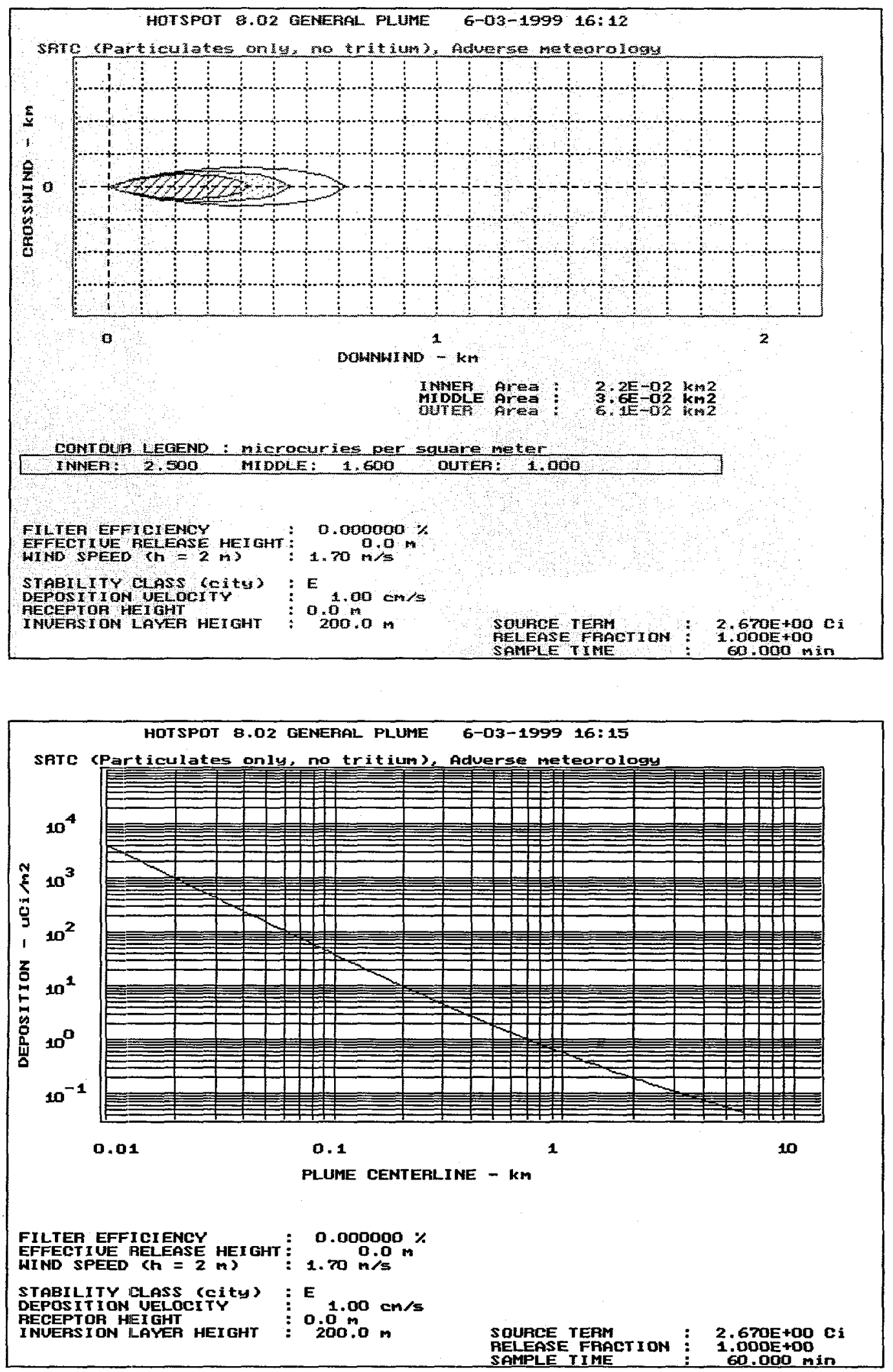


\section{Attachment G}

HOTSPOT results - adverse meteorology, $10 \mathrm{~cm} \mathrm{~s}^{-1}$ 

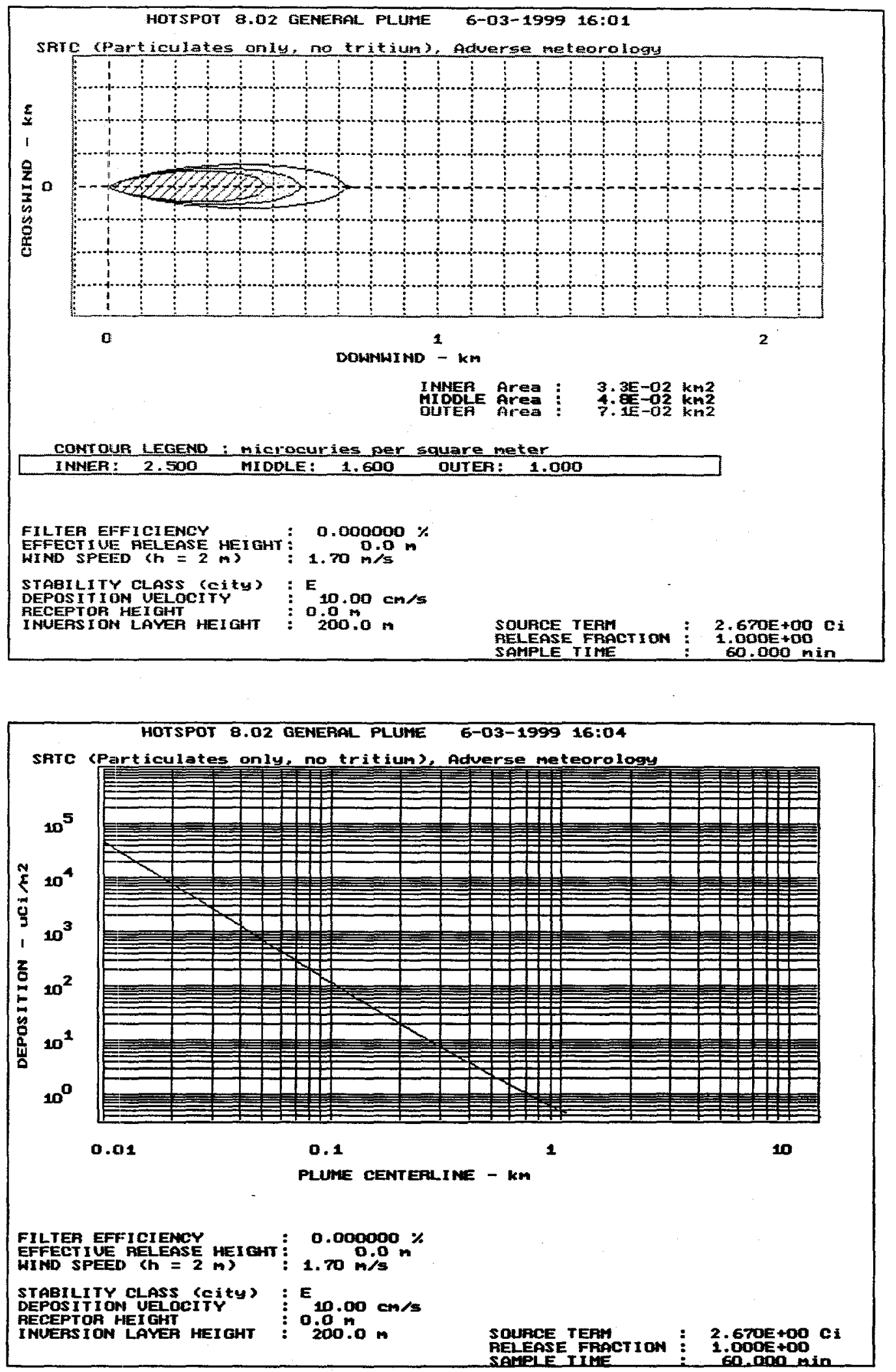